US Army Corps of Engineers $s_{\circledast}$ Engineer Research and Development Center

\title{
ERDG
}

Navigation Systems Research Program

Load and Resistance Factors for Earth Retaining, Reinforced Concrete Hydraulic Structures Based on a Reliability Index $(\beta)$ Derived from the Probability of Unsatisfactory Performance (PUP)

Phase 2 Study

Robert M. Ebeling and Barry C. White March 2021

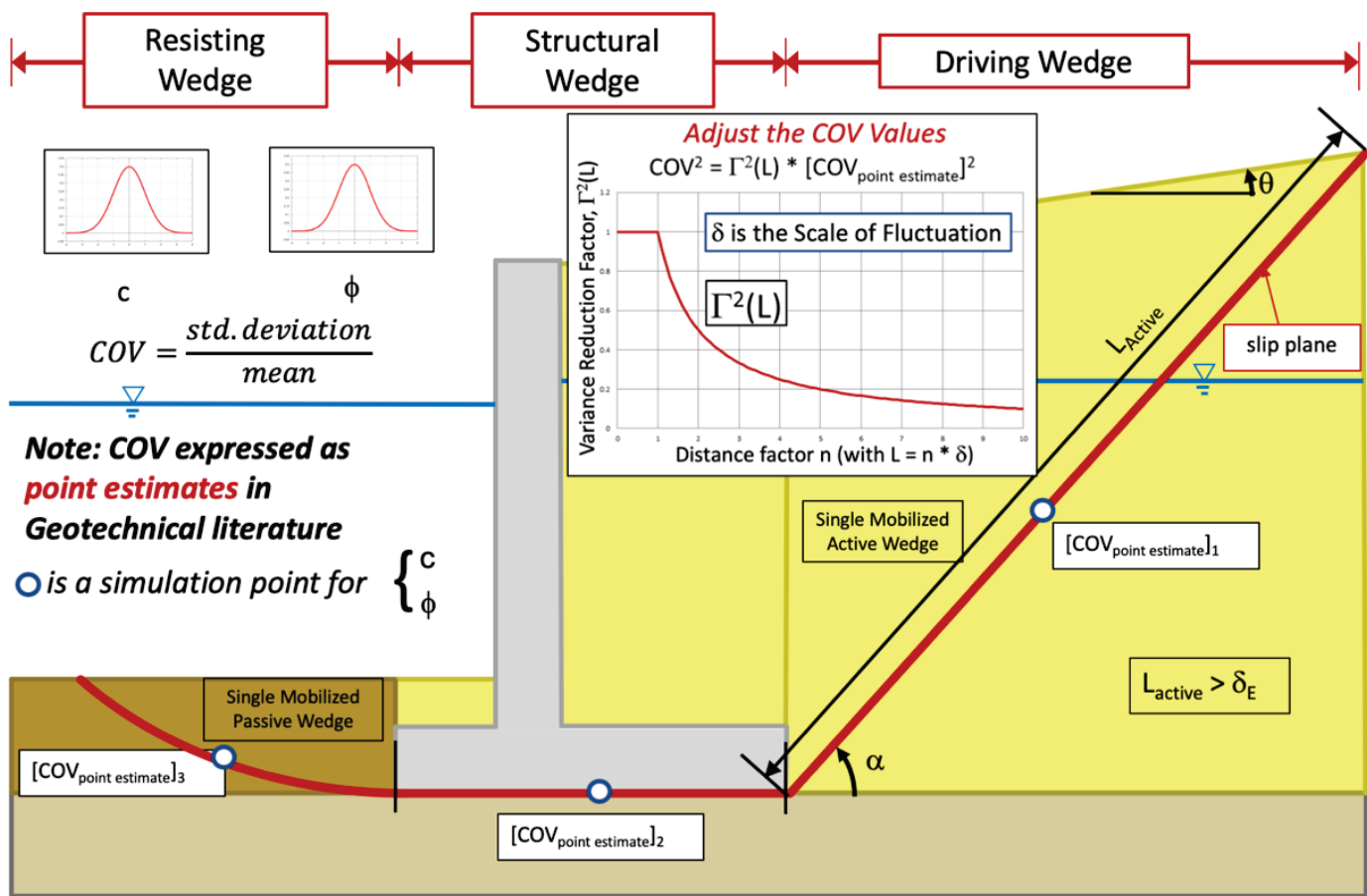


The U.S. Army Engineer Research and Development Center (ERDC) solves the nation's toughest engineering and environmental challenges. ERDC develops innovative solutions in civil and military engineering, geospatial sciences, water resources, and environmental sciences for the Army, the Department of Defense, civilian agencies, and our nation's public good. Find out more at www.erdc.usace.army.mil.

To search for other technical reports published by ERDC, visit the ERDC online library at https://erdclibrary.on.worldcat.org/discovery. 


\section{Load and Resistance Factors for Earth Retaining, Reinforced Concrete Hydraulic Structures Based on a Reliability Index $(\beta)$ Derived from the Probability of Unsatisfactory Performance (PUP)}

Phase 2 Study

Robert M. Ebeling and Barry C. White

Information Technology Laboratory

U.S. Army Engineer Research and Development Center 3909 Halls Ferry Road

Vicksburg, MS 39180-6199

Final report

Approved for public release; distribution is unlimited.

Prepared for Navigation Systems Research Program

Coastal and Hydraulics Laboratory

U.S. Army Engineer Research and Development Center

Vicksburg, MS 39180-6199

Under Funding Account Code U4375143; AMSCO Code 031391 


\section{Abstract}

This technical report documents the second of a two-phase research and development (R\&D) study in support of the development of a combined Load and Resistance Factor Design (LRFD) methodology that accommodates geotechnical as well as structural design limit states for design of the U.S. Army Corps of Engineers (USACE) reinforced concrete, hydraulic navigation structures. To this end, this R\&D effort extends reliability procedures that have been developed for other non-USACE structural systems to encompass USACE hydraulic structures. Many of these reinforced concrete, hydraulic structures are founded on and/or retain earth or are buttressed by an earthen feature. Consequently, the design of many of these hydraulic structures involves significant soil structure interaction. Development of the required reliability and corresponding LRFD procedures has been lagging in the geotechnical topic area as compared to those for structural limit state considerations and have therefore been the focus of this second-phase R\&D effort. Design of an example T-Wall hydraulic structure involves consideration of five geotechnical and structural limit states. New numerical procedures have been developed for precise multiple limit state reliability calculations and for complete LRFD analysis of this example T-Wall reinforced concrete, hydraulic structure.

DISCLAIMER: The contents of this report are not to be used for advertising, publication, or promotional purposes. Citation of trade names does not constitute an official endorsement or approval of the use of such commercial products. All product names and trademarks cited are the property of their respective owners. The findings of this report are not to be construed as an official Department of the Army position unless so designated by other authorized documents. 


\section{Contents}

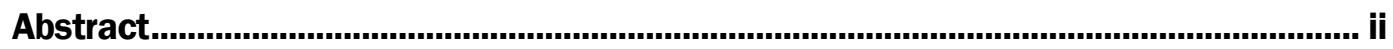

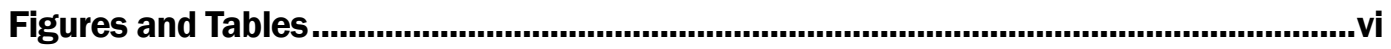

Preface

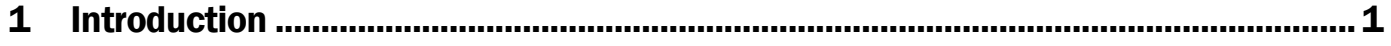

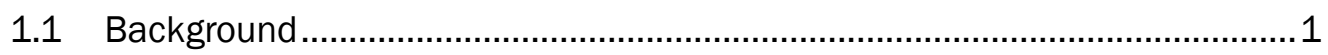

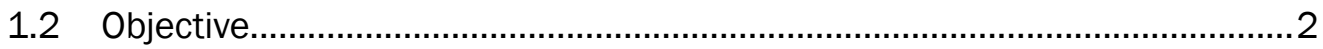

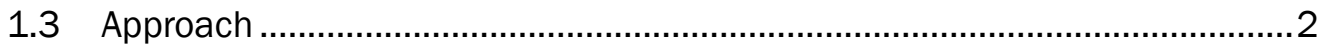

1.4 Overview of the translation from Reliability Index into a Load Factor .............3

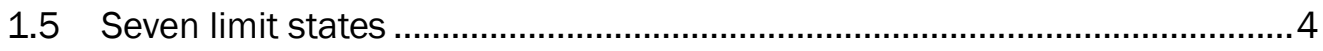

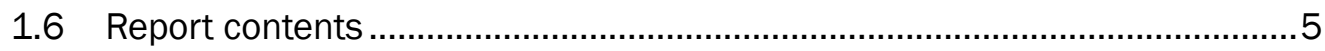

2 Building an Example Soil-Structure Interaction Model with Material

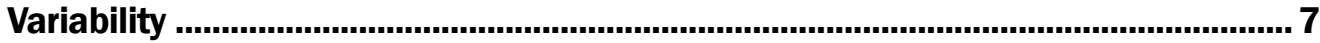

2.1 Three-wedge T-Wall SSI example problem ................................................. 7

2.2 Key reliability methodology relationships...................................................11

2.3 Key Geotechnical relationships being used to compute $P_{R}$ and $P_{L}$ forces acting on the Structural Wedge ................................................................16

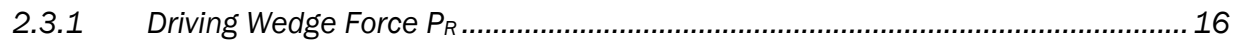

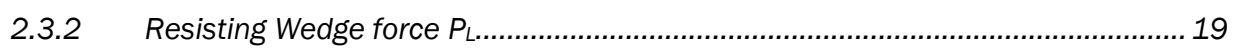

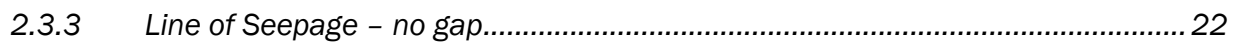

2.3.4 Line of Seepage - gap ........................................................................................ 25

2.4 Shear force, resultant normal force $N^{\prime}$ and its location along the base of the Structural Wedge ........................................................................27

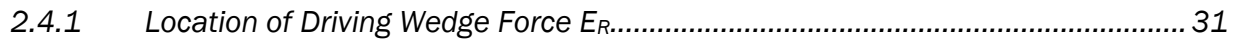

2.4.2 Location of horizontal water pressure resultant force - right side of the Structural Wedge .............................................................................................................. 33

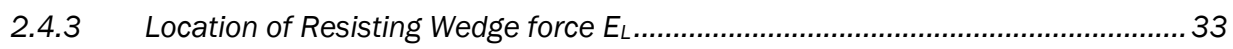

2.4.4 Location of horizontal water pressure resultant force - right side of the

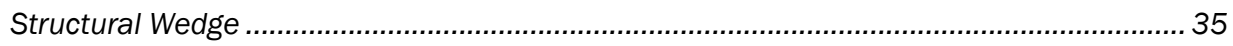

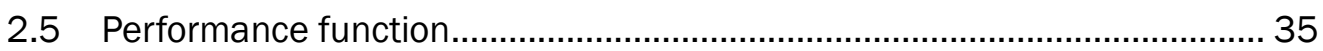

3 The Five Limit States Being Considered for the Example Soil-Structure Interaction of the T-Wall Model with Material Variability ....................................37

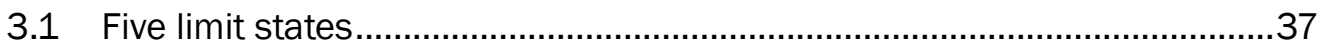

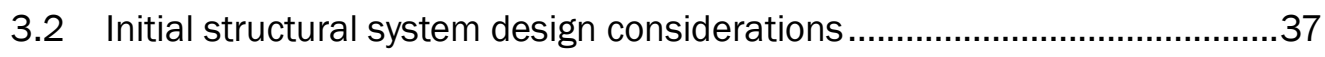

3.3 Base area in compression of the Structural Wedge limit state ................... 38

3.3.1 Iterative method for determining interactions between Driving, Resisting, and Structural Wedges for at-rest equilibrium conditions for Base Area in Compression limit state. 38

3.3.2 Engineering steps in the calculation of COVspatial for the Driving and Resisting Wedges and Foundation Soil..... 
3.3.3 Engineering steps to assess the performance

(satisfactory/unsatisfactory) of the Structural Wedge for the base area in

compression limit state

3.4 Global sliding failure of the Driving, Structural, and Resisting Wedge

limit state

3.4.1 Iterative method for determining interactions between Driving,

Resisting, and Structural Wedges for force equilibrium conditions for Sliding limit

state 45

3.4.2 Engineering steps in the calculation of COVspatial for the Driving and

Resisting Wedges and the Foundation Soil under the sliding limit state...

3.4.3 Engineering steps in the Reliability analysis of the Structural Wedge for the sliding limit state...

3.5 Bearing failure of the Structural Wedge limit state.....

3.5.1 Iterative method for determining interactions between Driving,

Resisting, and Structural Wedges for force equilibrium conditions for bearing limit

state 54

3.5.2 Engineering steps in the calculation of $\mathrm{COV}_{\text {spatial }}$ for the foundation soil ...............55

3.5.3 Engineering steps to assess the performance

(satisfactory/unsatisfactory) of the Structural Wedge for the bearing limit state

3.6 Shear failure of the wall stem limit state

3.7 Flexural failure of the wall stem limit state.

4 A Procedural Method for Computing Individual Limit State Probability of Unsatisfactory Performance (PUP) and Load Factors for the Example Soil-Structure Interaction of the T-Wall Model with Material Variability ................65

4.1 Introduction and research 65

4.2 Individual limit state procedure for computing load and resistance

distributions from material properties and determining load factors.

4.3 Sliding Limit State results for mid-range COVs ......................................... 73

4.4 Base Area in Compression Limit State results for mid-range COVs .............77

4.5 Bearing Limit State results for mid-range COVs ......................................... 78

4.6 Soil Structure Interaction (SSI)-proportioned load individual limit state procedure for computing load and resistance distributions from material properties and determining load factors

4.7 Using $R_{S F}$ and $L_{S F}$ to compute load factor $(\gamma L)$ and resistance factor

$\left(\varphi_{R}\right) \quad 85$

4.7.1 Computing load and resistance factors from $L_{S F}$.

4.7.2 Adjusting load and resistance factors to account for bias with nominal loads and resistances.

4.8 Using upper range point estimate coefficient of variation (COV) values for material properties.

4.9 Conclusions.

4.9.1 Results from Original, Mid-Range COV values for effective angles of friction 94

4.9.2 Results from Upper Range COV values for effective angles of friction 96

\section{A Numerical Method for Computing Multiple Limit States Probability of Unsatisfactory Performance (PUP) and Load Factors for the Example Soil- Structure Interaction of the T-Wall Model with Material Variability}




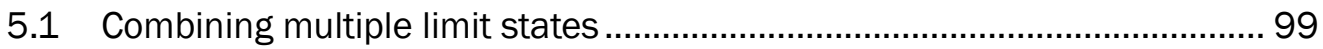

5.2 The multivariate space for the response function.................................. 101

5.3 Calculation methods for PUP ............................................................. 102

5.3.1 Advanced second moment (ASM) method for a single limit state ......................102

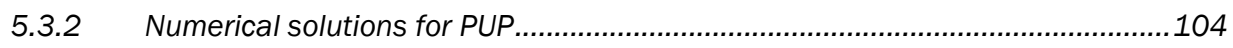

5.3.3 Computing PUP using simulation ...................................................................... 107

5.3.4 Finding the limiting range (bounds) for PUP......................................................109

5.4 Determining Load and Resistance Factor Design (LRFD) values from

PUP 115

5.5 Calculating PUP system for the Chapter 2 Geotechnical T-Wall example problem with a $25 \mathrm{ft}$ and $15 \mathrm{ft}$ base widths

5.6 Calculating PUP system for the Chapter 2 T-Wall Example Problem with Geotechnical and Structural Limit States using $25 \mathrm{ft}$ base width....

5.7 Checking sliding resistance scale factors against the five-limit state probability integration calculation .............................................................. 138

6 Summary, Conclusions, and Research Recommendations.................................... 144

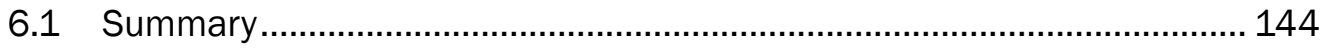

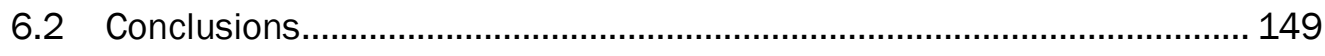

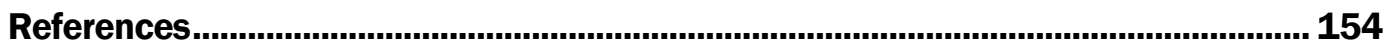

Appendix A: Computing Probabilities.................................................................... 158

Appendix B: Earth and Water Pressure Distributions, Resultant Forces and

Their Points of Application along the Faces of the Structural Wedge ................ 194

Appendix C: Numerical Methods for the Calculation of $\beta$ and Load and Resistance Factors Given Uncorrelated Load and Resistance PDF Curves........ 214

Appendix D: Calculations of Soil Bearing Capacity for Limit State Definition ........... 252

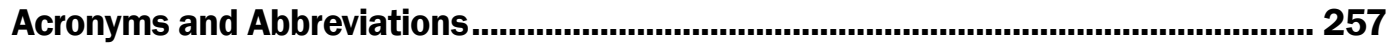

Report Documentation Page 


\section{Figures and Tables}

\section{Figures}

Figure 2-1. Diagram of an example soil-founded, reinforced concrete hydraulic structure for analysis.

Figure 2-2. Preliminary T-Wall geometry resulting from a CTWall design for a normal load case.

Figure 2-3. Driving Wedge and Resisting Wedge forces imposed on a Structural Wedge, $P_{R}$ and $P_{L}$.

Figure 2-4. Variance reduction function $\Gamma^{2}(L)$.

Figure 2-5. Geometrical relationships used to define the height terms in the equivalent unit weight relationship for a partially submerged soil wedge delineated by a planar slip surface.

Figure 2-6. Seepage path for gradient determination assuming full contact along the base of the Structural Wedge with its foundation.

Figure 2-7 1-D seepage path. (a) Constant slope in total head with distance along the transformed length of line of seepage in a homogenous, 1-D seepage path through all $K_{v-1}$ material. (b) Variation of slope in total head with distance along the three soil regions of the line of seepage.

Figure 2-8. Seepage path for gradient determination assuming base separation along a portion of the base of the Structural Wedge with its foundation, starting at the heel.

Figure 2-9. 1-D seepage path with base gap. (a) Constant slope in total head with distance along the transformed length of line of seepage in a homogenous, 1-D seepage path through all $\mathrm{K}_{\mathrm{v}-1}$ material. (b) Variation of slope in total head with distance along the three soil regions (and 1 region of gap) of the line of seepage.

Figure 2-10. Free body diagram of forces acting on the Structural Wedge, including the left- and right-hand side forces resulting from the Resisting Wedge and Driving Wedge, $P_{L}$ and $P_{R}$, respectively.

Figure 2-11. Horizontal component of effective earth pressures and water pressures with their corresponding resultant forces acting on the right side of the Structural Wedge.

Figure 2-12. Horizontal component of effective earth pressures and water pressures with their corresponding resultant forces acting on the left side of the Structural Wedge.

Figure 2-13. Capacity vs. demand related to the probability of unsatisfactory performance (after Nowak and Collins 2000, 2013).

Figure 3-1. Free body diagram of resultant forces acting on the stem and base slab of the Structural Wedge.

Figure 3-2. Idealizations of shear zones at failure of an earth-supported strip footing Structural Wedge. Zone I: Rankine Active zone; Zone II: Rankine Passive zone; and Zone III: Radial Shear zone.

Figure 4-1. Unscaled Load and Resistance Distributions and the resulting Gz()

distribution for the Sliding Limit State. 
Figure 4-2. An $R_{S F}$ Scale Factor of 1.734 is applied to the Resistance Distributions and a new $\mathrm{Gz}\left(\right.$ ) distribution is computed with $P U P_{\text {Target }}$ for the Sliding Limit State.

Figure 4-3. Representations of the four design cases: (a) Normal Load Case, (b) Dewatered Load Case, (c) Dewatered with Reduced Fill for the Resisting Wedge, and (d) Dewatered with No Fill for the Resisting Wedge.

Figure 4-4. Predicted resistances (using the Section 4.2 procedure) for multiple wall widths to achieve a target beta (with fixed load) versus the actual $\beta$ values computed for each wall width.

Figure 4-5. Predicted resistances for multiple wall widths to achieve a target $\beta$ target (with load adjusted to match the rate of change with the variable resistance) versus the actual $\beta$ values computed for each wall width.

Figure 4-6. Actual versus Predicted Resistance, Load, and R-L histograms for the $\beta$ target value of 2.9348 corresponding to the actual $\beta$ for the $25 \mathrm{ft}$ base width model.

Figure 4-7. Computed values for Sliding Limit State $L_{S F}$ with the same target $\beta$ (designated B) as the COVs go from the original, Mid-Range value ( $t=0.0)$ to the Upper Range values ( $t=1.0)$.

Figure 4-8. Computed values for Sliding Limit State Load Factor (based on a resistance factor of 0.5 ) with the same target $\beta(\mathrm{B})$ as the COVs go from the original, Mid-Range value ( $t=0.0$ ) to the Upper Range values $(t=1.0)$.

Figure 4-9. Upper Range COV value based computed Sliding Limit State Load Factors for increasing $\beta$ Target, all for a Resistance Factor of 0.5 .

Figure 5-1. A 2-D example of multiple limit states with closest approach of length

$|\beta|$ in the transformed basic variable space.

Figure 5-2. A 2-D example of the PUP region and the hyperplane description using $\alpha$ and $\beta$ determined using ASM methods. 103

Figure 5-3. Transferring the hyperplanar limit state to the single normal axis. 104

Figure 5-4. Numerical integration and computation of a PUP value with two variables with Gaussian distributions given a single hyperplanar limit state (using Euclidean coordinates).

Figure 5-5. Importance Sampling determines the ratio between two distributions $P$ and $Q$ to calculate the true $p$ probability for the point sampled from $q$.

Figure 5-6. Mapping the integrated PUP from the multivariate hyperplane to a single Gaussian distribution.

Figure 5-7. Mapping the integrated PUP from the multivariate hypersphere to a single Gaussian distribution.

Figure 5-8. Finding the overlap region A given limit state I with respect to limit state $\mathrm{J}$

Figure 5-9. Finding the overlap region B given limit state $J$ with respect to limit state I.

Figure 5-10. Setting radius $\beta$ for a hypersphere so the probability density outside the hypersphere integrates to the same value as the integration of the area region bounded by the limit state hyperplanes. 
Figure 5-11. Sample simulation sets of 3-D (Driving, Resisting, and Structural Wedge) material soil friction $\phi$ variable data drawn from the different sets of Normal distributions for each of the three limit states overlapped, given a T-Wall with base width of $25 \mathrm{ft}$.

Figure 5-12. Sample set of 3-D (Driving, Resisting, and Structural Wedge) material soil friction $\phi$ variable data drawn from Normal distributions for the Base Area in Compression Limit State, given a T-Wall with base width of $25 \mathrm{ft}$

Figure 5-13. Sample set of 3-D (Driving, Resisting, and Structural Wedge) material soil friction $\phi$ variable data drawn from Normal distributions for the Sliding Limit State with Satisfactory and Unsatisfactory performance points and ASM limit state hyperplane, given a T-Wall with base width of $25 \mathrm{ft}$.

Figure 5-14. Sample set of 3-D (Driving, Resisting, and Structural Wedge) material soil friction $\phi$ variable data drawn from Normal distributions for the Bearing Limit State with no Unsatisfactory performance points, given a T-Wall with base width of $25 \mathrm{ft}$.

Figure 5-15. Hybrid hyperspherical PUP system simulation results for a T-Wall under Extreme loads with base width of $25 \mathrm{ft}$.

Figure 5-16. Hybrid hyperspherical PUP system simulation results for a T-Wall under Usual loads with base width of $15 \mathrm{ft}$.

Figure 5-17. Individual limit state analysis of $\beta$ with respect to increasing $R_{S F}$. 136

Figure 5-18. Comparison of simulated Sliding Limit State, 5-D integration analysis for Sliding Limit State, and 5-D integration analysis with the combined limit state response $\beta$ values as $R_{S F}$ is increased.

Figure 5-19. Comparison of system $\beta$ values computed using increasing Sliding

$R_{\text {SF }}$ to the Dominant Sliding Limit State $\beta$ Target values........................................................ 140

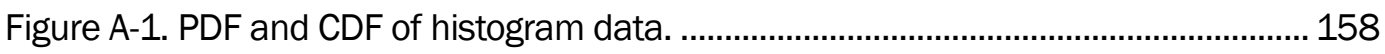

Figure A-2. Histogram data and the Gaussian (normal) distribution............................... 159

Figure A-3. The $G\left(X^{\prime}\right)$ function is used to determine PUP................................................ 159

Figure A-4. A 2-D example of the PUP region and the hyperplane description using $\alpha$ and $\beta$

Figure A-5. A 2-D example of multiple limit states with closest approach of length $|\beta|$

Figure A-6. Finding the contribution of area A to overlapping probabilities of $g_{i}(X)$ and $g_{j}(X)$.

Figure A-7. Finding the contribution of area $A$ to overlapping probabilities of $g_{i}(X)$ and $g_{j}(X)$.

Figure A-8. Gridding the Gaussian multivariate space to determine the PUP based on a single limit state response surface.

Figure A-9. Symmetric probability for pairs of bins equidistant from the mean, section view.

Figure A-10. Symmetric probability of the Gaussian Normal Curve at any distance in the multivariate space at all points on the hypersphere surface, plan view.

Figure A-11. A 2-D demonstration of the hypersphere calculation of PUP.

Figure A-12. Calculating dimensions for integrating spherical coordinate 3-D space. 
Figure A-13. Distribution plots with respect to radius for 3-D sphere: (a) sphere shell volume, (b) point distribution probability, (c) probability density for the sphere shell, and (d) number of divisions of the sphere density where each shell division would have 1.0e-10 probability.

Figure A-14. Example limit states and $\beta$ s for unfactored loads and resistances.

Figure A-15. A uniform scale factor $(S)$ is applied to the $\beta$ s for the limit state design points.

Figure A-16. Applying variable axis scale factors to adjust probabilities. A scale factor applied to Limit State 1 causes slope changes in the response surfaces for Limit State 2 and 3.

Figure A-17. The first test case for PUP estimation.

Figure A-18. The second test case for PUP estimation.

Figure B-1. Free body diagram of forces acting on the Structural Wedge, including the left- and right-side forces resulting from the Resisting Wedge and Driving Wedge, $P_{L}$ and $P_{R}$, respectively.

Figure B-2. Horizontal component of effective earth pressures and water pressures with their corresponding resultant forces acting on the right side of the Structural Wedge.

Figure B-3. Free body diagram of resultant forces acting on and within the soil block (right) located above the heel portion of the base slab for the Structural Wedge...

Figure B-4. Active and passive Rankine states of stress behind and in front of a cantilever retaining wall, respectively (after Peck et al. 1974).

Figure B-5. Horizontal component of effective earth pressures and water pressures with their corresponding resultant forces acting on the left side of the Structural Wedge.

Figure B-6. Free body diagram of resultant forces acting on and within the soil block (left) located above the toe portion of the base slab for the Structural Wedge.

Figure B-7. Free body diagram of resultant forces acting on the stem and base slab of the Structural Wedge.

Figure C-1. Reliability Index $\beta$ as a function of the constant $k$ for the problem of a reinforced concrete beam with its capacity defined by a nominal resisting moment of $311.11 \mathrm{kip}-\mathrm{ft}$ with a mean resisting moment of $348.44 \mathrm{kip}-\mathrm{ft}$.

Figure C-2. Reliability Index $\beta$ as a function of the constant $k$ for the problem of a reinforced concrete beam with its capacity defined by a nominal resisting moment of $337.68 \mathrm{kip}-\mathrm{ft}$ with a mean resisting moment of $378.31 \mathrm{kip}-\mathrm{ft}$.

Figure C-3. Computed safety margin PDF, PDF for a normal load PDF defined with a mean of $210 \mathrm{kip}$-ft, and a PDF for a lognormal resistance PDF defined by a nominal resisting moment of $311.11 \mathrm{kip}-\mathrm{ft}$ with a mean resisting moment of 348.44 kip-ft.

Figure C-4. Resulting simulation PDF for the $R$ - $L$ distribution given a lognormal resistance distribution (nominal resisting moment of $311.11 \mathrm{kip}-\mathrm{ft}$ and $\mathrm{COV}_{R}=$ 0.14 ) and a normal load distribution (nominal load moment of $200.00 \mathrm{kip}-\mathrm{ft}$ and $\left.\operatorname{COV}_{D L}=0.1\right)$. 
Figure C-5. Resulting simulation PDF for the $R$ - $L$ distribution (given nominal resisting moment of $311.11 \mathrm{kip}-\mathrm{ft}$ ) with curve fit by summing seven fractional normal curves.

Figure C-6. Resulting simulation PDF for the $R$ - $L$ distribution given a lognormal resistance distribution (nominal resisting moment of $337.68 \mathrm{kip}$-ft and $\mathrm{COV}_{R}=$ 0.14 ) and a normal load distribution (nominal resisting moment of $200.00 \mathrm{kip}-\mathrm{ft}$ and $\left.C O V_{D L}=0.1\right)$.

Figure C-7. Resulting simulation PDF for the $R$ - $L$ distribution (given nominal resisting moment of $337.68 \mathrm{kip}-\mathrm{ft}$ ) with curve fit by summing seven fractional normal curves.

Figure D-1. A geometry view of a complex T-Wall configuration with definition of the geometry necessary to compute bearing capacity.

\section{Tables}

Table 2-1. Engineering properties for the three soil regions of the example TWall problem.

Table 2-2. Vertical and horizontal scales of fluctuation for the three soil regions of the example T-Wall problem.

Table 2-3. Passive earth pressure coefficient $K_{\text {Passive }}(K p)$ values for $-\delta$ equal to $\varphi$ and a solution based on assuming a failure surface composed of logarithmic spiral portion and a planar surface portion - level ground.

Table 2-4. Reduction factor, $R$, values for various ratios of $-\delta / \phi$.

Table 3-1. Iterative at-rest procedure to calculate Soil Wedge interactions.

Table 3-2. Procedural method to calculate $\mathrm{COV}_{\text {spatial }}$ for the Driving and Resisting Wedges

Table 3-3. Base area in compression limit state procedure of Reliability analysis computing $g(X)$ per simulation value $X$.

Table 3-4. Iterative procedure to calculate equilibrium force Soil Wedge interactions.

Table 3-5. Procedure to calculate $\mathrm{COV}_{\text {spatial }}$ for the Driving and Resisting Wedges and for the foundation soil.

Table 3-6. Step-by-step sliding limit state procedure of Reliability analysis of the Structural Wedge.

Table 3-7. Step-by-step base area in compression limit state procedure of analysis of the Structural Wedge to calculate $\mathrm{COV}_{\text {spatial }}$ for the Driving and Resisting Wedges.

Table 3-8. Step-by step-base area in compression limit state procedure of

Reliability analysis of the Structural Wedge.

Table 3-9. Design resistance model for the concrete Shear limit state (after Ellingwood).

Table 3-10. Design resistance model for the flexural limit state (after Ellingwood)...........63

Table 4-1. For the $25 \mathrm{ft}$ wide base with Normal (watered) operating conditions:

$\mu_{R}=54,042.96 \mathrm{lb}, \mu L=38,963.16 \mathrm{lb}, \beta_{\text {initial }}=3.48, P U P_{\text {initial }}=0.00025, R[]$ to $L[]$

correlation $=0.976556$. 
Table 4-2. For the $25 \mathrm{ft}$ wide base with Extreme (dewatered) operating conditions:

$\mu_{R}=51,763.52 \mathrm{lb}, \mu L=37,574.50 \mathrm{lb}, \beta_{\text {initial }}=2.92, P U P_{\text {initial }}=0.00175, R[]$ to $L[]$

correlation $=0.978495$.

Table 4-3. For the $25 \mathrm{ft}$ wide base with beyond-Extreme soil to top of base (dewatered) operating conditions:

$\mu_{R}=43,459.34 \mathrm{lb}, \mu_{L}=34,874.95 \mathrm{lb}, \beta_{\text {initial }}=1.39, P U P_{\text {initial }}=0.08229, R[]$ to $L[]$

correlation $=0.973397$.

Table 4-4. For the $25 \mathrm{ft}$ wide base with beyond-Extreme scoured soil to the toe

of the structure (dewatered) operating conditions:

$\mu_{R}=42,775.71 \mathrm{lb}, \mu_{L}=34,427.89 \mathrm{lb}, \beta_{\text {initial }}=1.251, P U P_{\text {initial }}=0.10551, R[]$ to

$L[]$ correlation $=0.971258$.

Table 4-5. For the $25 \mathrm{ft}$ wide base with standard (watered) operating conditions:

$\mu_{R}=1,390,809.72 \mathrm{ft}-\mathrm{lb}, \mu_{L}=806,216.96 \mathrm{ft}-\mathrm{lb}, \beta_{\text {initial }}>6.0, P U P_{\text {initial }}=0.00000$,

$R[]$ to $L[]$ correlation $=0.298669$.

Table 4-6. For $25 \mathrm{ft}$ wide base with standard (dewatered) operating conditions:

$\mu_{R}=1,370,070.13 \mathrm{ft}-\mathrm{lb}, \mu \mathrm{L}=740,131.49 \mathrm{ft}-\mathrm{lb}, \beta_{\text {initial }}>6.0, P U P_{\text {initial }}=0.00000$,

$R[]$ to $L[]$ correlation $=0.331293$.

Table 4-7. For the $25 \mathrm{ft}$ wide base with standard (watered) operating conditions:

$\mu_{R}=131,936.98 \mathrm{lb}, \mu_{L}=48,736.99 \mathrm{lb}, \beta_{\text {initial }}>6.0, P U P_{\text {initial }}=0.00000, R[]$ to $L[]$

correlation $=0.880871$.

Table 4-8. For $25 \mathrm{ft}$ wide base with standard (dewatered) operating conditions:

$\mu_{R}=149,225.00 \mathrm{lb}, \mu_{L}=54,808.31 \mathrm{lb}, \beta_{\text {initial }}>6.0, P U P_{\text {initial }}=0.00000, R[]$ to $L[]$

correlation $=0.811645$.

Table 4-9. Computation of Load and Resistance scale factors with $\beta$ target equal to 3.5 for each of the Extreme load conditions.

Table 4-10. Engineering properties with Upper Range COV values for the three soil regions of the example T-Wall problem

Table 5-1. Mid-Range means and standard deviations for soil effective angle of internal friction $\phi$ ' values, in degrees, for the three different limit states and their three different soil regions along with their COV.

Table 5-2. Mid-Range means and standard deviations for soil effective angle of internal friction $\phi$ ' values, in degrees.

Table 5-3. Upper Range means and standard deviations for soil effective angle of internal friction $\phi$ ' values, in degrees.

Table 5-4. Means and standard deviations for stem shear force and stem moment.

Table 5-5. ASM results for the limit states of the 5-D integration problem 
Table 5-6. The results of applying the 5-D adaptive hyperspherical Gaussian probability numerical integration technique with the $R_{S F}$ values determined for Sliding $\beta$ Target values using Upper Range COVs.

Table 5-7. Examining the ratios of PUP and $\beta$ for Sliding limit state target and integrated 5-D system results.

Table 5-8. Targeted theoretical maximum lifetime (75-year) failure probabilities, $p_{m}$, and equivalent reliability indices, $\beta$, for the ULS in the 2014 CHBDC for foundations or geotechnical systems (after Fenton et al. 2016).

Table A-1. PUP values for Hyperplanar Limit State and Hypersphere PUP

Estimations with increasing accuracy.

Table A-2. Hypersphere PUP estimate error as a multiple of the actual Hyperplanar PUP.

Table C-1. Iterations of Step 3-8 as the design point and mean resistance change.

Table C-2. Iterations of Step 3-10 as the design point and $\beta$ values change. 231

Table C-3. Iterations of Step 3-10 as the design point and $\beta$ values change for the Hokens write-up of LRFD procedures.

Table C-4. Comparison of LRFD statistical variable parameters and computed results from the unpublished USACE (2016) letter report and the computations made in this appendix.

Table C-5. Reliability Index $\beta$ as a function of the constant $k$ for the problem of a reinforced concrete beam with its capacity defined by a nominal resisting moment of $311.11 \mathrm{kip}-\mathrm{ft}$ with a mean resisting moment of $348.44 \mathrm{kip}-\mathrm{ft}$.

Table C-6. Reliability Index $\beta$ as a function of the constant $k$ for the problem of a reinforced concrete beam with its capacity defined by a nominal resisting moment of 337.68 kip-ft with a mean resisting moment of 378.31 kip-ft. .

Table C-7. Computed values of series constant coefficients, means, standard deviations, and reliability indices for each of the seven Gaussian functions for the problem of a reinforced concrete beam with its capacity defined by a nominal resisting moment of 311.11 kip-ft with a mean resisting moment of 348.44 kip$\mathrm{ft}$.

Table C-8. Computed values of series constant coefficients, means, standard deviations and reliability indices for each of the seven Gaussian functions for the problem of a reinforced concrete beam with its capacity defined by a nominal resisting moment of $337.68 \mathrm{kip}-\mathrm{ft}$ with a mean resisting moment of $378.31 \mathrm{kip}-\mathrm{ft}$....... 245

Table C-9. Load and resistance factors and the effect on resistance.

Table C-10. Applying Section C.4 calculation of $\beta$ using the various load and resistance factors.

Table C-11. Calculating the maximum $\beta$ using the Section C.5 procedure given nominal resistances based on computed load and resistance factors.

Table C-12. Results of $10 \mathrm{M}$ simulations being performed using procedural load and resistance factors.

Table C-13. Results of 10M simulations being performed using procedural load and resistance factors. 


\section{Preface}

This study and report were authorized by the Navigation Systems (NavSys) Research Program, under Funding Account Code U4375143; AMSCO Code 031391. Mr. Charles E. Wiggins, U.S. Army Corps of Engineers (USACE), Coastal and Hydraulics Laboratory (CHL), was the Program Manager for the NavSys Research Program during a significant amount of time in which the majority of this research was conducted. From October 2017 through April 2018, Dr. Duncan B. Bryant, CHL, was acting Program Manager; from January through June of 2019, Dr. Patricia K. DiJoseph, CHL, was Acting Program Manager; from April through June of 2020, Dr. Katherine E. Brutsché, CHL, was Acting Program Manager; and from June 2020 through the present, Ms. Morgan Johnston was the Program Manager. At the time of preparation of this report, Mr. Kareem S. El-Naggar was the HQUSACE Navigation Business Line Manager.

The research was performed by the Computational Science and Engineering Division, U.S. Army Engineer Research and Development Center - Information Technology Laboratory (ERDC-ITL). At the time of publication of this report, Dr. Jerrell R. Ballard was, Chief. Mr. W. Jeff Lillycrop (ERDC-CHL) (retired) was the Navigation Technical Director until December 2018. Mr. Charles E. Wiggins has been Navigation Technical Director since January 2019 and during the time of publication of this report. The Deputy Director of ERDC-ITL was Ms. Patti S. Duett, and the Director was Dr. David A. Horner.

COL Teresa A. Schlosser was the Commander of ERDC, and the Director was Dr. David W. Pittman. 


\section{Introduction}

\subsection{Background}

The U.S. Army Corps of Engineers (USACE) has been relying on an allowable stress design (ASD) methodology for design of the various structural features comprising its hydraulic navigation structures. In ASD, also referred to as working stress design, factors of safety (FS) are implied through the use of allowable stresses during the structural design process. Allowable stresses are specified for use in the various design limit state conditions (flexural failure of a structural member, etc.) for the USACE three categories of design load cases of Usual, Unusual, and Extreme. ${ }^{1}$ In the USACE design guidance for geotechnical-dominated limit states and engineering issues, the use of FS against Ultimate Limit States (ULS) is more straightforward than in the Structural design guidance. The appropriate values for the FS are explicitly stated in this portion of the USACE guidance.

The USACE is currently transitioning to a structural design methodology based on Load and Resistance Factor Design (LRFD). The advantage for LRFD is that it formally takes into consideration the variability in the loads and the resistances separately. LRFD has become an accepted mode of implementation of probability-based limit state design in structural steel and reinforced concrete design. Three of the most recently developed and prominent examples of the LRFD-based building and bridge design criteria are

- American Society of Civil Engineers (2010), ASCE/SEI 7-10 Minimum Design Loads for Buildings and Other Structures

- Canadian Standards Association (CSA 2014), Canadian Highway Bridge Design Code, CAN/CSA-S6-14

- American Association of State Highway and Transportation Officials (AASHTO 2010), AASHTO LRFD Bridge Design Specifications.

\footnotetext{
1 One definition of a limit state is as a structural condition beyond which it no longer fulfills the specified design criteria.
} 
Development of these three LRFD-based design criteria have been led by the Structural engineering community within the civil engineering profession. In recent years, researchers have been proposing means for extending LRFD into Geotechnical ULS designs. However, to date, there are no LRFD-based Geotechnical design criteria.

Many of the USACE hydraulic structures are founded on and/or retain earth or are buttressed by an earthen feature. Consequently, the designs of many of the USACE hydraulic structures involve significant soil structure interaction (SSI). Generally speaking, SSI is a specialized topic associated with foundation features for the structure and with a structure's ability to retain earth and/or to be buttressed by an earthen mass. Currently, no LRFD methodology exists for SSI analysis of the USACE-type of hydraulic structures.

\subsection{Objective}

This technical report discusses research in support of the development of a Combined LRFD methodology that accommodates Structural as well as Geotechnical design limit states (especially the ULSs) as used in the design of the USACE hydraulic navigation structures. To this end, this research and development (R\&D) effort intends to extend, to the extent possible, reliability procedures that have been developed for other non-USACE structural systems to encompass USACE hydraulic structures. Additionally, procedural methods for reliability analysis are examined and evaluated as possible means for accurate estimation of reliability for application to the to-be-developed combined LRFD methodology.

\subsection{Approach}

The authors of this technical report have gathered applicable reliability methodologies and supporting data from the civil engineering LRFD technical community. These methodologies and data come from many structural and geotechnical fields and are interpreted for ultimate use in a combined LRFD application to the USACE hydraulic structures. Numerical procedures to facilitate accurate reliability calculations are also devised to accommodate reliability analysis of the USACE hydraulic structures' multiple limit states.

This technical report builds on the basic research into this topic as summarized in the Ebeling and White (2019) Phase 1 R\&D study. 
Specifically, this Phase 2 R\&D study fills in the technical gaps left out of the Phase 1 study as well as provides for a step-by-step approach to Reliability analysis of a reinforced concrete, hydraulic structure, including the supporting engineering analysis details, and translation into load factors. An evaluation of Reliability procedures will be performed in support of a methodology specification.

\subsection{Overview of the translation from Reliability Index into a Load Factor}

To statistically determine the capacity of a structural system, in general, the loads and resistances of the system need to be examined to find where the system will exceed the limitations for acceptable use. The probability for the exceedance of limitations is called the Probability of Unsatisfactory Performance (PUP), which is measured using Reliability methods. The use of specific Reliability methodology (advanced second moment [ASM] methods) allows for the determination of a reliability index $\beta$ (a scalar value) and the corresponding directional cosine $\alpha_{L}$ (a vector value) for the normally distributed loads in a multivariate space (Ebeling and White 2019). The definition of the load factor $\gamma_{L}$ (a vector value) for a single linear limit state can be determined directly from $\beta$ and $\alpha_{L}$ values in the following fashion:

$$
\gamma_{L}=\left(1+\alpha_{L} * \beta * \operatorname{COV}_{L}\right)
$$

where $C O V_{L}$ is the coefficient of variation (COV) $(=\sigma / \mu)$ of the load $L$. In the case of multiple limit states, $\beta$ and $\alpha_{L}$ values can be determined for each limit state. Similarly, the definition of the resistance factor $\varphi_{\boldsymbol{R}}$ (a vector value) for a single linear limit state can be determined directly from $\beta$ and $\alpha_{R}$ values using

$$
\varphi_{R}=\left(1-\alpha_{R} * \beta * \operatorname{COV}_{R}\right)
$$

where $C O V_{R}$ is the coefficient of variation $(=\sigma / \mu)$ of the resistance $R$. Describing $L$ with a lognormal distribution, Equation 1.1 becomes

$$
\gamma_{L}=e^{\alpha_{L} * \beta * \operatorname{COV}_{L}}
$$

and with a lognormal distribution for $R$, Equation 1.2 becomes

$$
\varphi_{R}=e^{-\alpha_{R} * \beta * \operatorname{COV}_{R}}
$$


The PUP value can be determined for non-linear or multiple limit states as a holistic system. This paper discusses a procedure to determine an appropriate set of values for $\beta$ and $\alpha_{L}$ given a system PUP value so that a corresponding holistic load factor $\gamma_{L}$ can be determined. In a Reliability analysis of piles in spatially varying soils and considering multiple limit states, Fan et al. (2014) demonstrated that the system PUP value may be underestimated if multiple limit states are considered separately. They conclude that this aspect of multiple limit states is one of the limitations of the current LRFD approach.

This application is in support of the design of USACE soil-founded, reinforced concrete hydraulic structures. Because these are soil-founded systems, variability in soil strength and earth loads can be extremely high requiring more precise calculation of the holistic system PUP value. Procedures for more precise calculation of the PUP for the multiple limit state system response are introduced. This technical report details the concluding R\&D with an application of the Reliability and SSI engineering methodologies devised during Phase 1 R\&D study to an example earth retaining structure.

\subsection{Seven limit states}

Soil-founded or rock-founded reinforced concrete hydraulic structures can possess up to seven limit states (Ebeling and White 2019). These limit states are

1. flexural failure of individual structural members

2. shear failure of individual structural members

3. buckling failure of individual structural members subjected to axial compressive loading

4. excessive displacement of the structural system in its entirety

5. global sliding failure of the structural system in its entirety

6. bearing failure of the foundation to the structural system in its entirety

7. overturning or rotational failure of the structural system about its foundation in its entirety.

The first three limit states are concerned with the reinforced concrete structural features. The fourth limit state is concerned with system performance. The latter three limit states are concerned with geotechnical effects on system response. 


\subsection{Report contents}

Chapter 2 discusses the hydraulic structure example problem, identifies the independent shear strength variables and their statistical distributions, and summarizes the engineering methodologies and equations needed for the engineering calculations used in the Reliability analysis.

Chapter 3 starts out by identifying the five limit states considered in the analysis. This chapter then proceeds to outline the step-by-step engineering methodologies for determining limit state conditions given interaction between the reinforced concrete hydraulic structures, the geotechnical features about the structure, and the loads acting on the structure.

Chapter 4 describes a Reliability-based procedural method for computing individual limit state PUP and Resistance and Load factors for the Chapter 2 example T-Wall model problem with soil shear-strength variability. A procedure will be introduced to compute load factors based on these Reliability calculations.

Chapter 5 outlines the step-by-step procedures for calculation of the PUP for soil-founded reinforced concrete hydraulic structures from a T-Wall system, point of view considering the five limit state methodologies of Chapter 3 .

Chapter 6 gives the summary and conclusions of this research study as well as recommendations on further research.

Appendix A discusses individual and multiple Limit States and the computation of the PUP using numerical procedures.

Appendix B describes the computation of earth and water pressure distributions, their resultant forces, and their points of application along the left and right faces of the Structural Wedge.

Appendix $\mathrm{C}$ describes numerical methods used for calibrating partial load and resistance safety factors given a target $\beta$ value. These numerical procedures require a mathematically defined, continuous probability density function (PDF) distributions for load and resistance as well as that they be non-correlated. The loads and resistances are modeled with distributions with at most two unknown means. Typically, the resistance variable accounts for one unknown mean with the other unknown mean 
corresponding to one of the load terms. Load ratios set the relationships of the mean values of the defined loads to the load with the unknown mean value. With these relationships, the ratio of the unknown mean value to the unknown resistance value allows for the definition of the load and resistance factors for the given target $\beta$. A supplemental procedure is derived for the calculation of the partial load safety factor given a specified partial resistance safety factor and the procedurally defined partial safety factor ratio. This supplemental procedure is valid for the condition where a single load and resistance variable are used. A second numerical procedure to calculate $\beta$ given variable load and resistance with distributions, which may have partial load and resistance safety factors applied is also presented. This second numerical procedure can be used as a verification step for the results from the first numerical procedure. An example for each of these procedures is included. The examples are taken from a 2016 USACE LRF ${ }^{1}$ study. Important details in the Reliability-based computational processes for load and resistance factor computations to provide for consistent results from the Appendix $\mathrm{C}$ numerical procedure and from the 2016 unpublished LRFD study ${ }^{1}$ are identified and explained.

Appendix D lists the equations required for establishing the limit state bearing capacity of a T-Wall founded on soil.

\footnotetext{
1 Kent D. Hokens. 2016 (Oct). Unpublished. USACE Load Factor Development for Design of Hydraulic Concrete Structures. St. Paul District. 


\section{Building an Example Soil-Structure Interaction Model with Material Variability}

\subsection{Three-wedge T-Wall SSI example problem}

Because uncertainty in the model is dominated by the geotechnical response of the system, an example problem will be developed that focuses on this response. It is convenient to devise an example retaining wall structural problem containing the three limit state features of interest: a global sliding failure, a foundation bearing failure, and an overturning or rotational failure of the structural system about its foundation. This example should not be so complex as to impede the analysis with geologic details such as those stemming from patterns of rock jointing, etc. This example retaining structure selected for study is shown in Figure 2-1 and consists of a T-wall retaining a soil backfill behind its heel, founded on a geologically formed soil and with a soil buttress in front of its toe that is also geologically formed.

Figure 2-1. Diagram of an example soil-founded, reinforced concrete hydraulic structure for analysis.

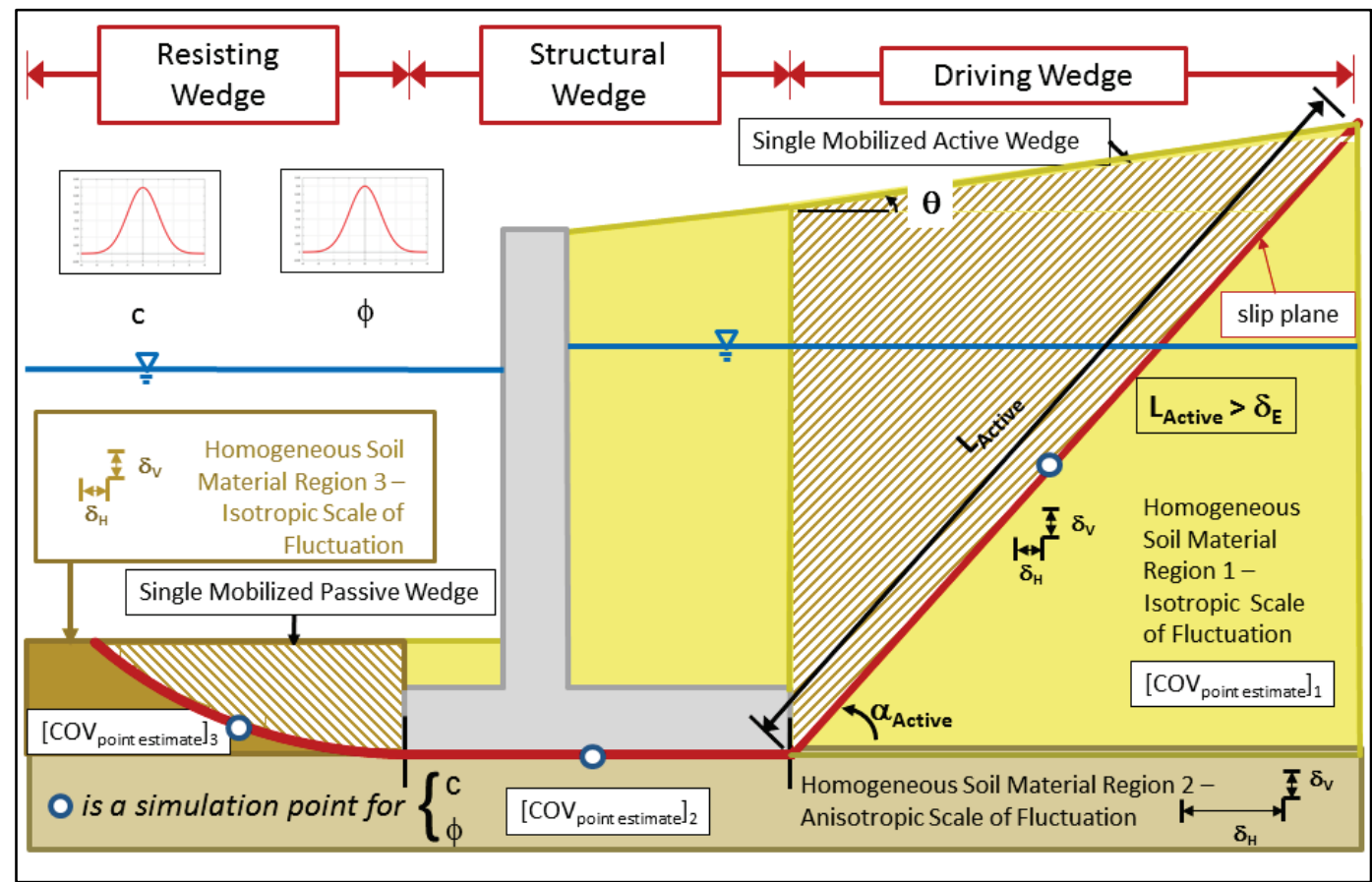

It possesses a pool of water to the left-hand side of the T-Wall and above the soil buttress. A water table is contained within the retained soil on the right-hand side of the T-Wall. A differential head condition exists for this 
T-Wall due to the existence of a higher head on the retained side of the wall relative to the pool elevation on the left-hand side of the wall. There are three soil regions identified in this figure, designated as soil regions 1, 2, and 3. Soil regions 1 (yellow) and 3 (gold) are engineer-selected backfill material placed by earth-moving equipment and compacted to engineering specification after construction of the T-Wall. Soil region $2(\tan )$ is naturally occurring soil and is assumed to be undisturbed by the construction of the T-Wall and backfill placement above it. Soil backfill is placed and compacted in front, behind, and above the T-Wall shortly after T-Wall construction and (reinforced) concrete curing concluded.

The engineering soil properties and their statistical distributions are summarized in Table 2-1. Effective stress engineering soil parameters are specified for each of the three soil regions. In this table, the mean value is represented using the Greek symbol $\mu$ while its coefficient of variation is designated by Mid-Range COV. Mean values for the Mohr-Coulomb shear strength, effective angle of internal soil friction values are provided, along with its Mid-Range point estimate COV value (in percent). Note that these are point estimate values and based upon the data summarized in Chapter 3 and Appendix D of Ebeling and White (2019). The soil strength parameter $\phi$ ' for the three soil regions is assumed to be normally distributed with a lower bound at $\mu$ minus $3^{*} \sigma$ and an upper bound at $\mu$ plus $5^{*} \sigma$, where $\sigma$ is the standard deviation ( $=\operatorname{COV}$ times $\mu$ ). An additional requirement is that the lower bound is never less than zero deg. The soils of the three regions are assumed to be cohesionless (i.e., $c^{\prime}$ equal to zero). Interface friction values are specified between the Driving and Structural Wedges (reported in the row labeled Soil region 1); between the base of the Structural Wedge and its foundation soil (reported in the row labeled Soil region 2); and between the Resisting and Structural Wedges (reported in the row labeled Soil region 3). Interface friction $\delta$ values are expressed as a fraction of the $\phi$ ' value in Table 2-1, assuming a very high degree of correlation between $\delta$ and $\phi$ '. In all probabilistic simulation analyses discussed in this report, $\phi^{\prime}$ values are varied according to the bounded normal PDF distribution while each $\delta$ value per $\phi$ 'value simulation is based upon the ratio of $\delta$ to $\phi^{\prime}$ as prescribed in this table. Mean hydraulic conductivity values are also reported along with its applicable direction of flow: vertical (V) or horizontal (H). 
Table 2-1. Engineering properties for the three soil regions of the example T-Wall problem.

\begin{tabular}{|c|c|c|c|c|c|c|c|c|c|}
\hline \multicolumn{2}{|c|}{} & \multicolumn{2}{|c|}{ Unit Weights } & \multicolumn{2}{c|}{$\begin{array}{c}\text { Soil Friction, } \\
\phi^{\prime}\end{array}$} & \multicolumn{2}{|c|}{$\begin{array}{c}\text { Interface } \\
\text { Friction, } \delta^{\prime}\end{array}$} & \multicolumn{2}{l|}{ Hydraulic Conductivity } \\
\hline $\begin{array}{c}\text { Soil } \\
\text { Region }\end{array}$ & Soil Type & $\begin{array}{c}\gamma_{\text {moist }} \\
(\mathrm{pcf})\end{array}$ & $\begin{array}{c}\gamma_{\text {sat }} \\
(\mathrm{pcf})\end{array}$ & $\begin{array}{c}\mu_{\phi^{\prime}} \\
(\mathrm{deg})\end{array}$ & $\begin{array}{c}\text { Mid- } \\
\text { Range } \\
\mathrm{COV}_{\phi^{\prime}} \\
(\%)\end{array}$ & $\begin{array}{c}\text { Ratio } \\
\delta^{\prime} / \phi^{\prime}\end{array}$ & $\begin{array}{c}\mu_{\delta^{\prime}} \\
(\mathrm{deg})\end{array}$ & $\begin{array}{c}\mu_{\mathrm{k}} \\
(\mathrm{cm} / \mathrm{sec})\end{array}$ & Direction \\
\hline $\mathbf{1}$ & $\begin{array}{c}\text { Compacted } \\
\text { sand }\end{array}$ & 123 & 126 & 35 & 10 & 0.4 & 14 & $2 \times 10^{-3}$ & Vertical \\
\hline $\mathbf{2}$ & $\begin{array}{c}\text { Silty sand } \\
\text { foundation }\end{array}$ & - & 123 & 30 & 20 & 1.0 & 30 & $4 \times 10^{-4}$ & Horizontal \\
\hline 3 & $\begin{array}{c}\text { Compacted } \\
\text { sand }\end{array}$ & - & 126 & 37 & 12 & 1.0 & 37 & $1 \times 10^{-3}$ & Vertical \\
\hline
\end{tabular}

The height of the retained soil relative to the left-hand soil and pool elevation dictates the right-hand side to be the driving-load side. The righthand side retained soil attempts to move the retaining structure towards the pool. A potential sliding plane is depicted in red in the Figure 2-1 cross section. This postulated retaining structure possesses all but one (i.e., buckling failure) of the seven limit states.

In preparation for engineering evaluation, the Figure 2-1 retaining wall system is divided into the Driving Wedge, the Structural Wedge, and the Resisting Wedge. This division of the soil features into three global wedges is consistent with the USACE EM 1110-2-2502 (HQUSACE 1989) design procedure of retaining wall analysis.

On the right-hand side of the retaining structure is the Driving Wedge, as labeled in Figure 2-1. Gravity causes the soil mass located above the potential slip plane in this wedge to slide downward along the (red) potential slip surface. This, in turn, generates lateral movement of the Structural Wedge towards the pool and results in "mobilized active" earth pressures acting against the Driving Wedge to Structural Wedge interface.

In the center of this figure is the Structural Wedge. The Structural Wedge consists of the retaining structure and any other soil fill material that lies contained within an area defined by the width of the structure. For this example, the potential slip plane is contained within the foundation soil (region 2) below the reinforced concrete structure.

On the left-hand side of the structure is the Resisting Wedge. In this wedge, the weight of the soil and other (i.e., pool of water) loads/weights are 
providing "mobilized passive" earth pressure against the structural wedge, therefore resisting sliding of the structural wedge. The Pool region extending above the top of the Resisting Wedge's ground surface also exerts a hydrostatic water pressure force acting horizontally on the Structural Wedge's left-hand side interface. This triangular hydrostatic water pressure distribution is not depicted in this figure. The slip plane in this region is typically more complicated than in the active pressure region. In the case of Figure 2-1, the slip plane of the resisting wedge is modeled using a logarithmic spiral curve. This is in contrast to the planar surface of the mobilized active driving wedge. Earth pressures are determined from a logarithmic spiral based slip plane solution and using resulting passive earth pressure coefficient, Kassive, tabulated by NAVFAC 7.2 (Department of the Navy 1982).

Due to its contact with retained earth, the majority of the USACE hydraulic structures face SSI issues. SSI is sometimes described in general terms as the process in which the response of the soil influences the structural movement, and this structural movement, in turn, influences the soil's response.

A CTWall (Pace 1994) analysis for the normal load case following EM 11102-2502 (HQUSACE 1989) design criteria (using Allowable Stress Design criteria) was conducted to develop preliminary T-Wall geometry using the mean values for the engineering soil properties given in Table 2-1.

Figure 2-2 summarizes the resulting T-Wall geometry. 
Figure 2-2. Preliminary T-Wall geometry resulting from a CTWall design for a normal load case.

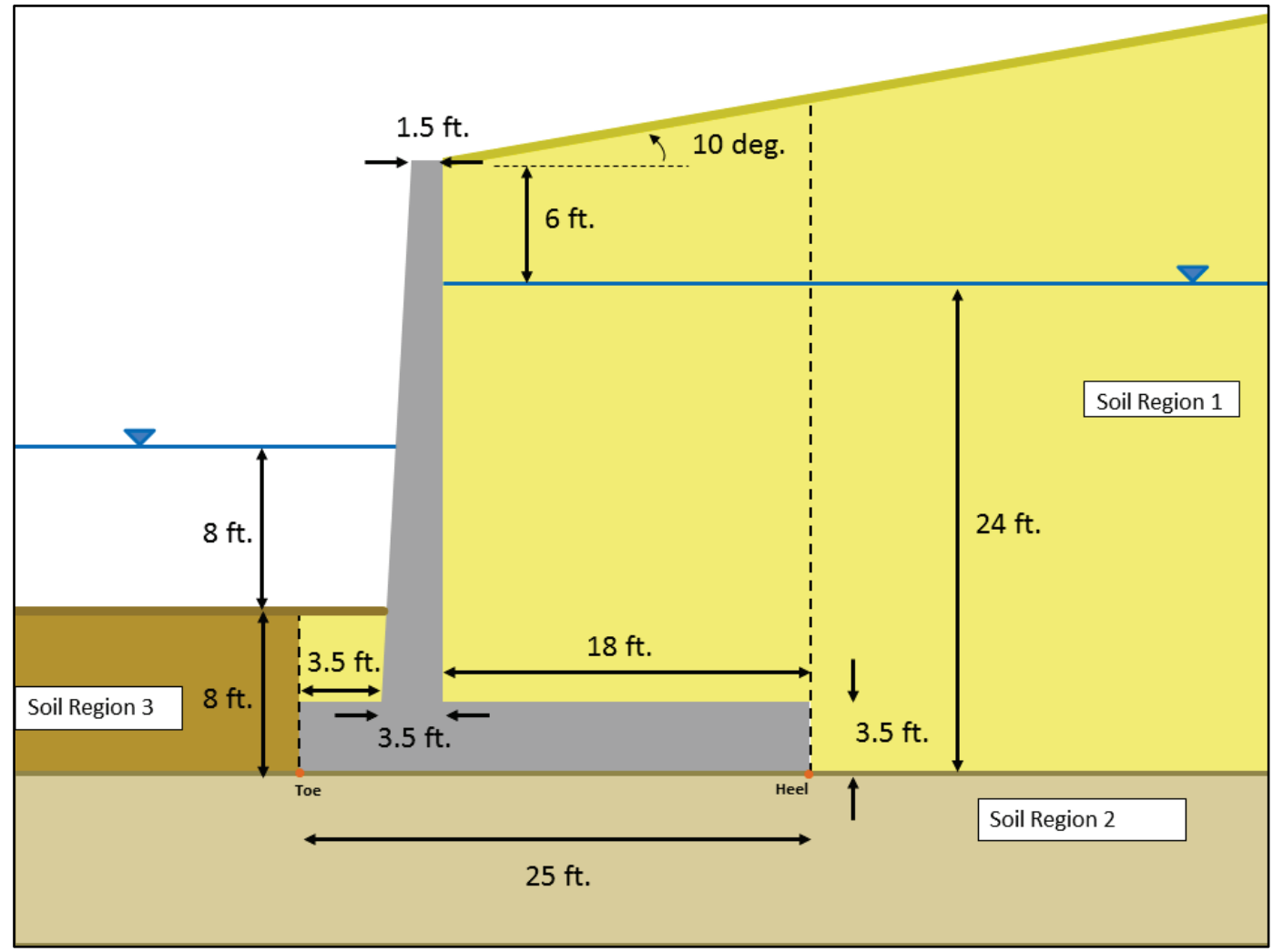

\subsection{Key reliability methodology relationships}

Ebeling and White (2019) devised three means for computing the Figure 2-1 statistical distributions for resultant earth pressure forces of the Figure 2-3 left-hand side $\left(P_{L}\right)$ and right-hand side $\left(P_{R}\right)$ resultant forces imposed on the Structural Wedge. These forces, $P_{L}$ and $P_{R}$, correspond to the reactions of the Resisting and Driving Wedges, respectively. 
Figure 2-3. Driving Wedge and Resisting Wedge forces imposed on a Structural Wedge, $P_{R}$ and $P_{L}$.

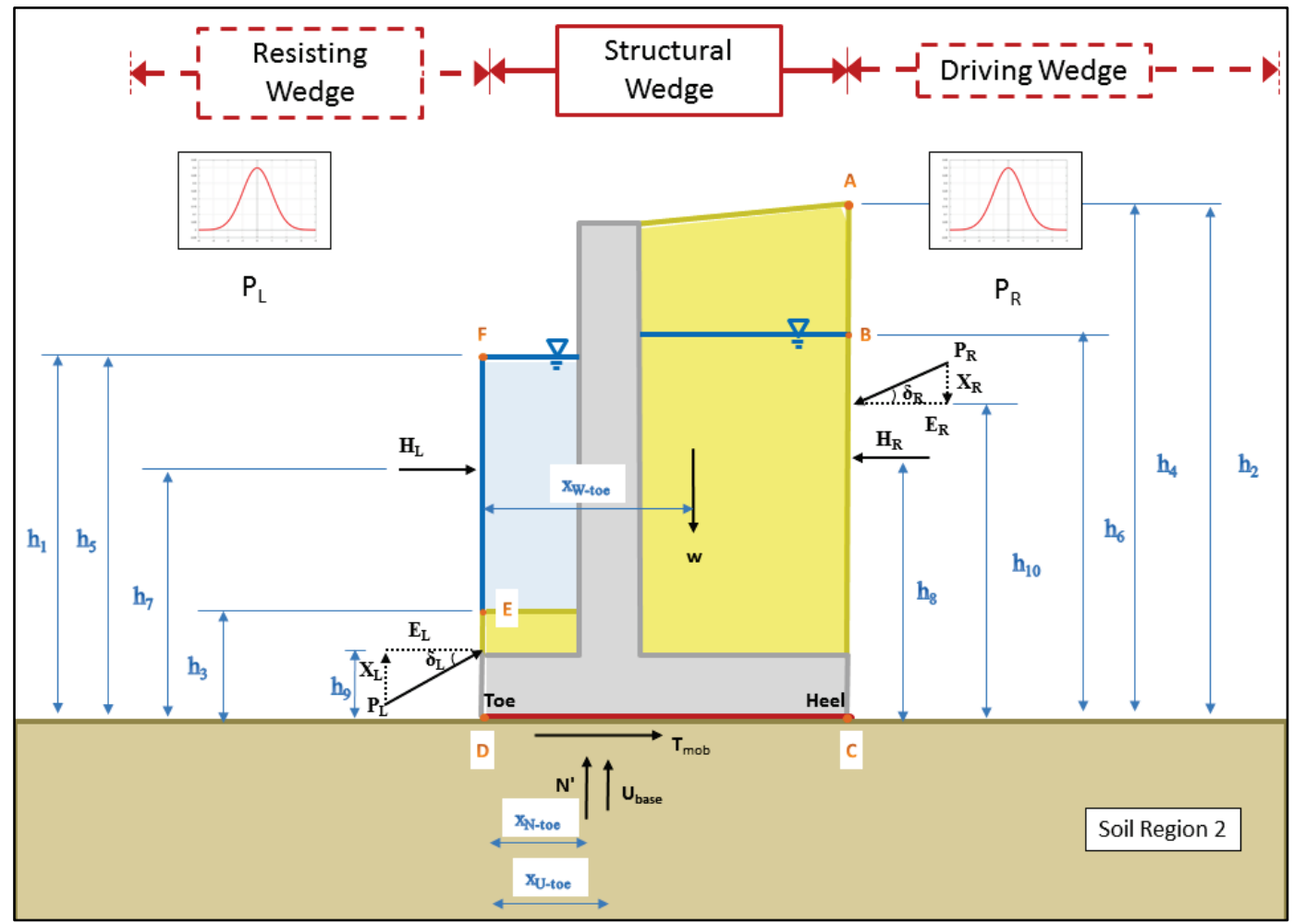

This T-Wall example uses two devised geostatistical variance methods methodologies, one for the Driving Wedge and the second for the Resisting Wedge. The two applied methodologies are as follows:

1. A sliding stability assessment using a wedge solution method that accounts for the reduction of correlation due to distances greater than the Scale of Fluctuation $(\delta)$ within the Driving Wedge combined with a simulation methodology to transfer uncertainty in the Mohr-Coulomb soil shear strength parameters (of $c$ and $\phi$ ) to the Figure 2-3 $P_{L}$ and $P_{R}$ resultant forces acting on the Structural Wedge. This procedure minimizes the number of wedges in the solution by using the Variance Reduction Factor $\Gamma^{2}\left(L_{\text {Active }}\right)$ in a method described later in this paper. LPassive is the length of the slip plane that defines the base of the Driving Wedge. The application of this procedure requires homogenous material within the Driving Wedge. This procedure is applied to the foundation soil below the Structural Wedge as well since it is also homogenous. Each of these two (Wedge) regions is comprised of different soil types at the location of the potential slip plane.

2. An earth pressure coefficient-based method of analysis combined with a simulation methodology to transfer uncertainty in the Mohr-Coulomb 
soil shear strength parameters directly to the Figure 2-3 $P_{L}$ resultant force $P_{L}$ acting on the Structural Wedge. This procedure is also restricted to homogenous soil in the area of the Resisting Wedge. The orientation of the slip planes is estimated using the Mohr-Coulomb soil shear strength parameters for determining the magnitude of the Variance Reduction Factor, $\Gamma^{2}\left(L_{\text {Passive }}\right)$, where LPassive is the length of the slip plane that defines the base of the Resisting Wedge.

El-Ramly et al. (2002a,b) and others observe that by taking the spatial average of the geotechnical design property (e.g., strength) variable over the whole area of interest, such as along the slip surface in a limit equilibrium sliding stability analysis, positive and negative random errors at different locations within the averaging domain tend to cancel out. As a result, the random error variance associated with the averaged soil strength quantity is largely reduced over point estimates (Vanmarcke 1977a,b, 1980). This reduction in variance over a point estimate's variance is why it is so important to account for variance reduction due to spatial correlation of soil properties in probabilistic geotechnical engineering analyses. The Variance Reduction Function $\Gamma^{2}(L)$ developed by Vanmarcke $(1977 \mathrm{a}, \mathrm{b})$ is a fundamental part of this approach.

$$
\Gamma^{2}(\mathrm{~L})= \begin{cases}1 & \mathrm{~L} \leq \delta \\ \frac{\delta}{\mathrm{L}} & \mathrm{L} \geq \delta\end{cases}
$$

where $\mathrm{L}$ is the length of the potential slip plane and $\delta$ is the scale of fluctuation of the soil region. This function is plotted in Figure 2-4. The spatial variability is described by the scale of fluctuation, which gives the distance at which soil engineering properties have changed enough to no longer be highly correlated. 
Figure 2-4. Variance reduction function $\Gamma^{2}(L)$.

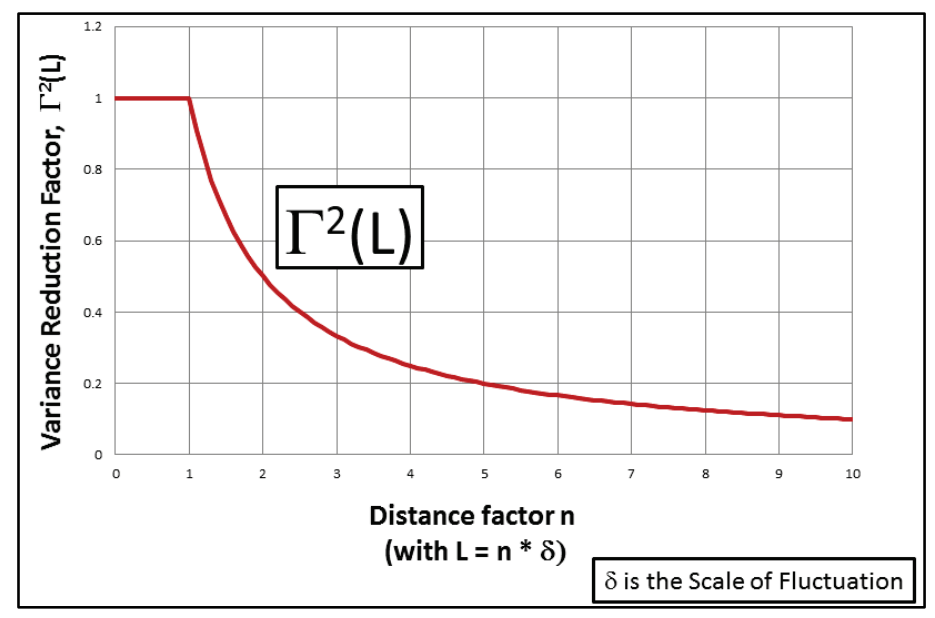

The coefficient of variation for $C O V_{\text {spatial }}$ of the spatial average variable of the soil Geotechnical design property as

$$
C O V_{\text {spatial }}^{2}=\Gamma^{2}(L) * C O V_{\text {point estimate }}^{2}+\operatorname{COV}_{\text {measurement error }}^{2}
$$

Recall that the COV is equal to the standard deviation for the variable divided by its mean value $(=\sigma / \mu)$. Values for the inherent variability of

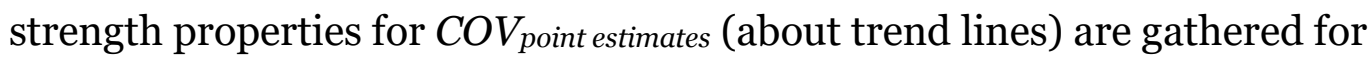
different soil types using laboratory and in situ strength tests while $C O V_{\text {measurement error }}$ reflects the variability in test results used to define the soil (strength) property. Historical data on both are given in Chapter 3 of Ebeling and White (2019).

The application of a variance reduction factor applied to $C O V_{\text {point estimates for }}$ soil strength is an important aspect of a Reliability analysis that includes Geotechnical limit state(s). Resulting values for $C O V_{\text {spatial }}$ estimates are less than $C O V_{\text {point estimates }}$ due to spatial averaging of the engineering strength parameters of the soil along specific planes, especially when the plane lengths are greater than the scale of fluctuation. These planes are usually selected by the engineer to be coincident with likely potential slip planes within the soil mass during a Reliability analysis considering Geotechnical Limit States.

The Geotechnical strength data for natural soil deposits formed over geologic time clearly demonstrate anisotropic scale of fluctuation values in the horizontal and vertical directions; $\delta_{V}$ and $\delta_{H}$. Appendix D of Ebeling and White (2019) provides a data base of values for $\delta_{V}$ and $\delta_{H}$, as well as the 
ratio of $\delta_{H} / \delta_{V}$. These data were obtained from the statistical processing of shear strength data with (vertical and horizontal) distance in various soil types. Over the last 20 years, engineers researching the spatial correlation of engineering soil properties have collected and studied the vertical and horizontal scale of fluctuation (or autocorrelation) engineering soil property data and some have made generalized observations. Several of these generalizations that have been published in the technical literature have been collected in Appendix D of Ebeling and White (2019) as well.

The Figure 2-1 T-Wall of interest envisions three regions of soil types, as discussed previously. For soil regions 1 (in yellow) and 3 (in gold) that consists of select engineered compacted soil, isotropic scales of fluctuation in the vertical and horizontal directions are hypothesized for the backfill. This is because during excavation of borrow material and its subsequent compaction as an engineered backfill destroys the original structural makeup of the soil due to the original geologic process and creates a new intermixed soil structure. This contrasts with the soil region 2 (in tan), which is assumed to be a naturally occurring soil deposit that has been formed through geologic means and on a geologic time scale. For soil region 2, $\delta \mathrm{h}$ is assumed to be greater than $\delta v$. According to the strength data in Appendix D of Ebeling and White (2019), the trend may be on the order of a factor of between 9 to 13 times greater. $\delta h$ is assumed to be 9 times $\delta_{v}$ for soil region 2 in this T-Wall example. The data collected in Ebeling and White (2019) indicate that $\delta v$ generally falls within a range of 0.5 to $2 \mathrm{~m} .{ }^{1,2} \mathrm{In}$ this T-Wall example, $\delta v$ is assumed to be equal to $0.5 \mathrm{~m}$ for all three soil regions. These $\delta_{v}$ and $\delta h$ data are summarized in Table 2-2 for the three soil regions.

\footnotetext{
1 For a full list of the spelled-out forms of the units of measure used in this document, please refer to US Government Publishing Office Style Manual, 31st ed. (Washington, DC: US Government Publishing Office 2016), 248-52, https://www.govinfo.gov/content/pkg/GPO-STYLEMANUAL-2016/pdf/GPOSTYLEMANUAL-2016.pdf.

2 For a full list of the unit conversions used in this document, please refer to US Government Publishing Office Style Manual, 31st ed. (Washington, DC: US Government Publishing Office 2016), 345-7, https://www.govinfo.gov/content/pkg/GPO-STYLEMANUAL-2016/pdf/GPO-STYLEMANUAL-2016.pdf.
} 
Table 2-2. Vertical and horizontal scales of fluctuation for the three soil regions of the example T-Wall problem.

\begin{tabular}{|c|c|c|c|c|c|c|}
\hline \multicolumn{2}{|c|}{} & \multicolumn{2}{|c|}{$\delta_{v}$} & \multicolumn{2}{c|}{$\delta_{n}$} & $\delta_{h} / \delta_{v}$ \\
\hline $\begin{array}{c}\text { Soil } \\
\text { Region }\end{array}$ & Soil Type & $(\mathrm{m})$ & $(\mathrm{ft})$ & $(\mathrm{m})$ & $(\mathrm{ft})$ & \\
\hline $\mathbf{1}$ & $\begin{array}{c}\text { Compacted } \\
\text { sand }\end{array}$ & 1 & 3.3 & 1 & 3.3 & 1 \\
\hline $\mathbf{2}$ & $\begin{array}{c}\text { Silty sand } \\
\text { foundation }\end{array}$ & 1 & 3.3 & 9 & 29.5 & 9 \\
\hline $\mathbf{3}$ & $\begin{array}{c}\text { Compacted } \\
\text { sand }\end{array}$ & 1 & 3.3 & 1 & 3.3 & 1 \\
\hline
\end{tabular}

As a result of the geologic process within a given layer for naturally deposited soils, soil properties tend to be anisotropic and, specifically, more variable in the vertical direction than in the horizontal direction. For these layers, a single scale of fluctuation value is needed for use along the potential slip plane when its orientation is neither horizontal nor vertical. Vanmarcke (1980) suggested an elliptical representation for estimation of an equivalent scale of fluctuation, $\delta E$, given a slip plane along the angle $\alpha$ from horizontal.

$$
\delta_{E}=\sqrt{\delta_{v}^{2} \sin ^{2} \alpha+\delta_{h}^{2} \cos ^{2} \alpha}
$$

Other proposed $\delta_{E}$ approximations are given in Appendix E of Ebeling and White (2019).

\subsection{Key Geotechnical relationships being used to compute $P_{R}$ and $P_{L}$ forces acting on the Structural Wedge}

\subsubsection{Driving Wedge Force $P_{R}$}

For a homogenous cohesionless retained soil, the thrust force that the Driving Wedge imposes on the Structural Wedge (designated $P_{R}$ in Figure 2-3) is computed using the active earth pressure force $\left(P_{A}\right)$ relationship

$$
P_{A}=K_{\text {Active }} * \frac{1}{2} * \gamma_{e} *\left(H_{\text {heel }}\right)^{2}
$$

$H_{\text {heel }}$ is the vertical height of the Structural to Driving wedge interface as measured from the heel of the T-Wall. $K_{\text {Active }}$ is the active earth pressure coefficient by the Coulomb's active earth pressure coefficient (Ebeling and 


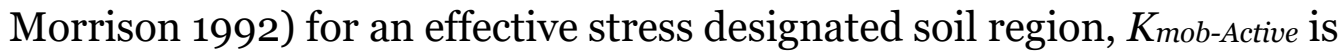
computed as

$$
K_{\text {mob-Active }}=\frac{\cos ^{2}\left(\phi^{\prime} \text { mob-Active }\right)}{\cos \left(\delta \prime^{\prime} \text { mob-Active }\right)\left[1+\sqrt{\frac{\sin \left(\phi^{\prime} \text { mob-Active }+\delta^{\prime} \text { mob-Active }\right) * \sin \left(\phi^{\prime} \text { mob-Active }-\theta\right)}{\cos \left(\delta^{\prime} \text { mob-Active }\right) * \cos (\theta)}}\right]^{2}}
$$

where $\phi^{\prime}$ mob-Active is the mobilized effective angle of internal friction of the soil and $\delta^{\prime}$ mob-Active is the mobilized interface angle of friction. The use of Equation 2.5 is restricted to cases in which the value of $\phi^{\prime}$ mob-Active is greater than $\theta$. In an effective stress analysis, the mobilized effective angle of internal friction $\phi^{\prime}$ mob-Active is given by

$$
\tan \left(\phi^{\prime}{ }_{\text {mob-Active }}\right)=\frac{\tan \left(\phi^{\prime}\right)}{F S_{\text {Active }}}
$$

The mobilized effective angle of interface friction for the Structural to Driving active wedges interface is given by

$$
\tan \left(\delta_{\text {mob-Active }}^{\prime}\right)=\frac{\tan \left(\delta^{\prime}\right)}{F S_{\text {Active }}}
$$

An equivalent unit weight $\gamma_{e}$ is used in Equation 2.4 to accommodate a water table within the retained soil of the Driving Wedge. In an effective stress analysis, a moist soil unit weight is assigned above the water table, and a saturated unit weight is assigned to the soil below the water table. Ebeling and White (2019) define the equivalent unit weight $\gamma_{e}$ as

$$
\gamma_{e}=\gamma_{1} *\left[\frac{h_{1}}{\left(h_{1}+h_{2}\right)+h_{3}} * \frac{h_{1}}{\left(h_{1}+h_{2}\right)}\right]+\gamma_{2-3} *\left[1-\frac{h_{1}}{\left(h_{1}+h_{2}\right)+h_{3}} * \frac{h_{1}}{\left(h_{1}+h_{2}\right)}\right]
$$

for the Figure 2-1 Driving Wedge possessing a planar slip plane oriented at an angle $\alpha$ from horizontal, with a saturated unit weight $\gamma_{\text {sat }}$ assigned to $\gamma_{1}$ for soil area 1 below the water table and a moist unit weight $\gamma_{\text {moist }}$ assigned to $\gamma_{2-3}$ for soil area 2-3 above the water table (Figure 2-5). The ground surface is oriented at an angle of $\theta$ from horizontal. 
Figure 2-5. Geometrical relationships used to define the height terms in the equivalent unit weight relationship for a partially submerged soil wedge delineated by a planar slip surface.

$H=h_{1}+h_{2}$
$\left(H+h_{3}\right)=H \cdot\left[\frac{\cos \theta \cdot \cos \alpha}{\sin (\alpha-\theta)}\right] \cdot \tan \alpha$
$h_{3}=\left(H+h_{3}\right)-H$
$h_{3}=H \cdot\left\{\left[\frac{\cos \theta \cdot \cos \alpha}{\sin (\alpha-\theta)}\right] \cdot \tan \alpha-1\right\}$

A moist unit weight $\gamma_{\text {moist }}$ was assigned to $\gamma_{2-3}$ for soil area 2-3 above the water table. When there is a differential head of water existing from one side of the Structural Wedge to the other, there will be flow of water within the soil regime. For the differential head example shown later in Figure 2-6, water will flow from the high-head side of the Driving Wedge side, through the foundation and up into the low-head side of the Resisting Wedge. To account for the effect of steady state seepage acting downward on the saturated volume of soil contained within the Driving Wedge, $\gamma_{1}$ for soil area 1 is

$$
\gamma_{1}=\gamma_{b u y}+\gamma_{w a t e r} * i_{y-D W}
$$

with $i_{y-D W}$ being the downward, vertical gradient of steady-state seepage through the saturated soil volume of the Driving Wedge. The buoyant unit weight rouy is defined as

$$
\gamma_{\text {buy }}=\gamma_{\text {sat }}-\gamma_{w a t e r}
$$

with $\gamma$ water designating the unit weight of water.

The orientation of a planar Driving Wedge slip plane (Ebeling and Morrison 1992), as measured from horizontal, is

$$
\alpha_{m o b-A c t i v e}=\phi_{m o b-A c t i v e}^{\prime}+\tan ^{-1}\left[\frac{-\tan \left(\phi^{\prime} m o b-A c t i v e-\theta\right)+c_{E 1}}{c_{E 2}}\right]
$$


where

$$
c_{E 1}=\left\{\begin{array}{c}
{\left[\tan \left(\phi^{\prime}{ }_{\text {mob-Active }}-\theta\right)\right] *} \\
{\left[\tan \left(\phi_{\text {mob-Active }}^{\prime}-\theta\right)+\cot \left(\phi_{\text {mob-Active }}^{\prime}\right)\right] * \text { term }_{E 1}}
\end{array}\right\}^{\frac{1}{2}}
$$

with

$$
\operatorname{term}_{E 1}=\left[1+\tan \left(\delta^{\prime}{ }_{\text {mob-Active }}\right) * \cot \left(\phi_{\text {mob-Active }}^{\prime}\right)\right]
$$

and

$$
c_{E 2}=1+\left\{\begin{array}{c}
{\left[\tan \left(\delta^{\prime}{ }_{\text {mob-Active }}\right)\right] *} \\
{\left[\tan \left(\phi_{\text {mob-Active }}^{\prime}-\theta\right)+\cot \left(\phi_{\text {mob-Active }}^{\prime}\right)\right]}
\end{array}\right\}
$$

The use of Equation 2.11 is restricted to cases in which the value of $\phi^{\prime}$ mobActive is greater than $\theta$. $\alpha_{\text {mob-Active }}$ is used to compute the value of $h_{3}$ (Figure 2-5) so that an equivalent unit weight may be determined using Equation 2.8. Computations made using Equation 2.11 in support of Equation 2.8 have restricted the minimum value of $\phi^{\prime}$ mob-Active to be equal to $\theta$ plus $2 \mathrm{deg}$. $\alpha_{\text {mob-Active }}$ is also used to compute the length of the Figure 2-1 slip plane $L$ Active for the Driving Wedge when determining the value of the Variance Reduction Factor $\Gamma^{2}\left(L=L_{\text {Active }}\right)$. Computations made using Equation 2.11 in support of $L_{\text {Active }}$ for the Driving Wedge have also restricted the minimum value of $\alpha_{\text {mob-Active }}$ to $\theta$ plus $2 \mathrm{deg}$. The equivalent unit weight Equation 2.8 is sensitive to the Figure 2-5 $h_{3}$ height, which can be excessively large for values of $\alpha$ that approach the value for $\theta$ because of the infinite length shear plane issue (refer to Figure 2-5).

\subsubsection{Resisting Wedge force $P_{L}$}

For a homogenous cohesionless butressing soil, the $P_{P}$ thrust force that the Resisting Wedge imposes on the Structural Wedge (designated $P_{L}$ in Figure 2-3) is computed using

$$
P_{P}=K_{\text {Passive }} * \frac{1}{2} * \gamma_{e} *\left(H_{\text {toe }}\right)^{2}
$$

$H_{\text {toe }}$ is the vertical height of the Structural to Resisting Wedge soil interface as measured from the toe of the T-Wall. The passive earth pressure coefficient $K_{\text {Passive }}$ is obtained from Table 5-1 in Ebeling and White (2019) 
for level ground and Resisting to Structural Wedge interface friction value of $\delta$ int. This table is reproduced as Table 2-3 below. The values of passive earth pressure coefficient were computed using Logarithmic Spiral slip surface-based relationships for level ground and $\delta=\delta_{\text {int }}$ and equal to $\phi$.

$$
K_{\text {Passive }}(\delta)=K_{\text {Passive }} \cdot R(\delta, \phi)
$$

Values for $R$ are provided in Ebeling and White (2019) Table 5-2 as a function of the value of $\phi$ and the value of the ratio $\delta / \phi$. This table is reproduced as Table 2-4.

Table 2-3. Passive earth pressure coefficient $K_{\text {Passive }}$ (Kp) values for $-\delta$ equal to $\varphi$ and a solution based on assuming a failure surface composed of logarithmic spiral portion and a planar surface portion - level ground.

\begin{tabular}{|c|c|}
\hline$\phi$ & $\mathrm{Kp}(-\delta=\phi)$ \\
\hline 0 & 1 \\
\hline 5 & 1.28 \\
\hline 10 & 1.64 \\
\hline 15 & 2.19 \\
\hline 20 & 3.01 \\
\hline 25 & 4.29 \\
\hline 30 & 6.42 \\
\hline 35 & 10.2 \\
\hline 40 & 17.5 \\
\hline 45 & 33.5 \\
\hline 50 & 74.3 \\
\hline 51 & 90 \\
\hline 52 & 110 \\
\hline 53 & 130 \\
\hline 54 & 160 \\
\hline 55 & 204 \\
\hline 60 & 782 \\
\hline
\end{tabular}


Table 2-4. Reduction factor, $R$, values for various ratios of $-\delta / \phi$.

\begin{tabular}{|c|c|c|c|c|c|c|c|c|c|c|c|}
\hline \multirow{2}{*}{$\phi$ (deg) } & \multicolumn{11}{|c|}{$\delta / \phi$} \\
\hline & -1 & -0.9 & -0.8 & -0.7 & -0.6 & -0.5 & -0.4 & -0.3 & -0.2 & -0.1 & 0 \\
\hline 0 & 1 & 1 & 1 & 1 & 1 & 1 & 1 & 1 & 1 & 1 & 1 \\
\hline 5 & 1 & 0.996 & 0.995 & 0.989 & 0.981 & 0.973 & 0.9645 & 0.956 & 0.949 & 0.9405 & 0.932 \\
\hline 10 & 1 & 0.991 & 0.989 & 0.978 & 0.962 & 0.946 & 0.929 & 0.912 & 0.898 & 0.881 & 0.864 \\
\hline 15 & 1 & 0.986 & 0.979 & 0.961 & 0.934 & 0.907 & 0.881 & 0.854 & 0.83 & 0.803 & 0.775 \\
\hline 20 & 1 & 0.983 & 0.968 & 0.939 & 0.901 & 0.862 & 0.824 & 0.787 & 0.752 & 0.716 & 0.678 \\
\hline 25 & 1 & 0.980 & 0.954 & 0.912 & 0.86 & 0.808 & 0.759 & 0.711 & 0.666 & 0.62 & 0.574 \\
\hline 30 & 1 & 0.980 & 0.937 & 0.878 & 0.811 & 0.746 & 0.686 & 0.627 & 0.574 & 0.52 & 0.467 \\
\hline 35 & 1 & 0.980 & 0.916 & 0.836 & 0.752 & 0.674 & 0.603 & 0.536 & 0.475 & 0.417 & 0.362 \\
\hline 40 & 1 & 0.980 & 0.886 & 0.783 & 0.682 & 0.592 & 0.512 & 0.439 & 0.375 & 0.316 & 0.262 \\
\hline 45 & 1 & 0.979 & 0.848 & 0.718 & 0.6 & 0.5 & 0.414 & 0.339 & 0.276 & 0.221 & 0.174 \\
\hline 50 & 1 & 0.975 & 0.797 & 0.638 & 0.506 & 0.399 & 0.313 & 0.242 & 0.185 & 0.138 & 0.102 \\
\hline 55 & 1 & 0.966 & 0.731 & 0.543 & 0.401 & 0.295 & 0.215 & 0.153 & 0.108 & 0.0737 & 0.0492 \\
\hline 60 & 1 & 0.948 & 0.647 & 0.434 & 0.29 & 0.193 & 0.127 & 0.0809 & 0.0505 & 0.0301 & 0.0178 \\
\hline
\end{tabular}

The mobilized effective angle of soil friction within the Resisting Wedge is given by

$$
\tan \left(\phi_{\text {mob-Passive }}^{\prime}\right)=\frac{\tan \left(\phi^{\prime}\right)}{F S_{\text {Passive }}}
$$

and the mobilized effective angle of interface friction for the wedge interface between Structural to Resisting passive wedges is

$$
\tan \left(\delta_{\text {mob-Passive }}^{\prime}\right)=\frac{\tan \left(\delta^{\prime}\right)}{F S_{\text {Passive }}}
$$

The following computation in this procedure is specific to the Example Problem of this report, where the Resisting Wedge has a level ground surface that is submerged (Figure 2-1). For the saturated volume of soil contained within the Resisting Wedge, the equivalent unit weight $\gamma_{e}$ for its soil area below the water table is

$$
\gamma_{e}=\gamma_{1}=\gamma_{b u y}-\gamma_{w a t e r} * i_{y-R W}
$$

with $i_{y-R W}$ being the upward, vertical gradient of steady-state seepage through the saturated soil volume of the Resisting Wedge. Note the sign change on the gradient terms between Equations 2.9 and 2.19, reflecting the influence of the downward and upward seepage, respectively.

The orientation of a planar slip plane (Ebeling and Morrison 1992), as measured from horizontal, is 


$$
\alpha_{m o b-P a s s i v e}=\left\{\begin{array}{c}
-\phi_{\text {mob-Passive }}^{\prime}+ \\
\tan ^{-1}\left[\frac{\tan \left(\phi^{\prime} \text { mob-Passive }+\theta\right)+c_{E 3}}{c_{E 4}}\right]
\end{array}\right\}
$$

where

$$
c_{E 3}=\left\{\begin{array}{c}
{\left[\tan \left(\phi_{\text {mob-Passive }}^{\prime}+\theta\right)\right] *} \\
{\left[\tan \left(\phi_{\text {mob-Passive }}^{\prime}+\theta\right)+\cot \left(\phi^{\prime}{ }_{\text {mob-Passive }}\right)\right] * \operatorname{term}_{E 2}}
\end{array}\right\}^{1 / 2}
$$

with

$$
\operatorname{term}_{E 2}=\left[1+\tan \left(\delta_{\text {mob-Passive }}^{\prime}\right) * \cot \left(\phi_{\text {mob-Passive }}^{\prime}\right)\right]
$$

and

$$
c_{E 4}=1+\left\{\begin{array}{c}
{\left[\tan \left(\delta_{\text {mob-Passive }}^{\prime}\right)\right] *} \\
{\left[\tan \left(\phi_{\text {mob-Passive }}^{\prime}+\theta\right)+\cot \left(\phi_{\text {mob-Passive }}^{\prime}\right)\right]}
\end{array}\right\}
$$

The exact geometry of the logarithmic spiral slip planes for the Table 2-3 data is not known. This $\alpha_{\text {mob-Passive }}$ relationship is used to compute an approximate length of the Passive Resisting Wedge slip plane LPassive for the Resisting Wedge when determining the value of the Variance Reduction Factor $\Gamma^{2}(L)$. It is recognized that a planar slip surface approximation is more accurate for soils with $\delta$ mob-Passive less than or equal to $\phi_{\text {mob-Passive }} / 2$, as discussed in Subsection 3.3.4.1 of Ebeling and Morrison (1992) or Section 4.6 of Chen and Liu (1990).

Computations made using Equation 2.20 in support of an approximate length computation of the Passive Resisting Wedge slip plane L Lassive for the Resisting Wedge have restricted the minimum value of $\alpha_{\text {mob-Passive }}$ to be equal to 5 deg for this level ground problem of a fully submerged Resisting Wedge. In this case, the computation of the equivalent unit weight by Equation 2.19 is not influenced by $\alpha_{m o b}$-Passive, as was the case for the Driving Wedge (with $\alpha_{\text {mob-Active }}$ ).

\subsubsection{Line of Seepage - no gap}

Water will travel from high (total) head (i.e., $H_{B}$ in Figure 2-6) to low (total) head $\left(H_{E}\right)$, dictating the direction of flow within the soil regions. Consequently, the side with the highest (total) head dictates that the direction of flow is downward along that side of the T-Wall. In a Line of 
Seepage analysis (aka, Line of Creep), a vertical streamline, or flow line, is assumed within the soil and adjacent to the Structural Wedge (Ebeling and White 2019). Full contact along the base of the Structural Wedge with its foundation is assumed in Figure 2-6. The transformation procedure for the line of seepage method is for one-dimensional (1-D) flow along a singular path consisting of 1-D flow paths connected in series from one soil region on into the next soil region that are aligned along the three faces of the Structural Wedge of a T-Wall. These flow paths contained within the soil regions may possess different values for the saturated hydraulic conductivity of the soil. The transformed lengths for each of the three faces are determined using the relationships given in Figure 2-7. Assigning the total head boundary conditions of $H_{B}$ at point $\mathrm{B}$ and $H_{C}$ point $\mathrm{C}$ and with a linear head drop along the transformed section in Figure 2-7(a) allows for the determination of total heads $H_{C}$ at point $\mathrm{C}$ and $H_{D}$ at point $\mathrm{D}$, as described in detail in Appendix G of Ebeling and White (2019).

Figure 2-6. Seepage path for gradient determination assuming full contact along the base of the Structural Wedge with its foundation.

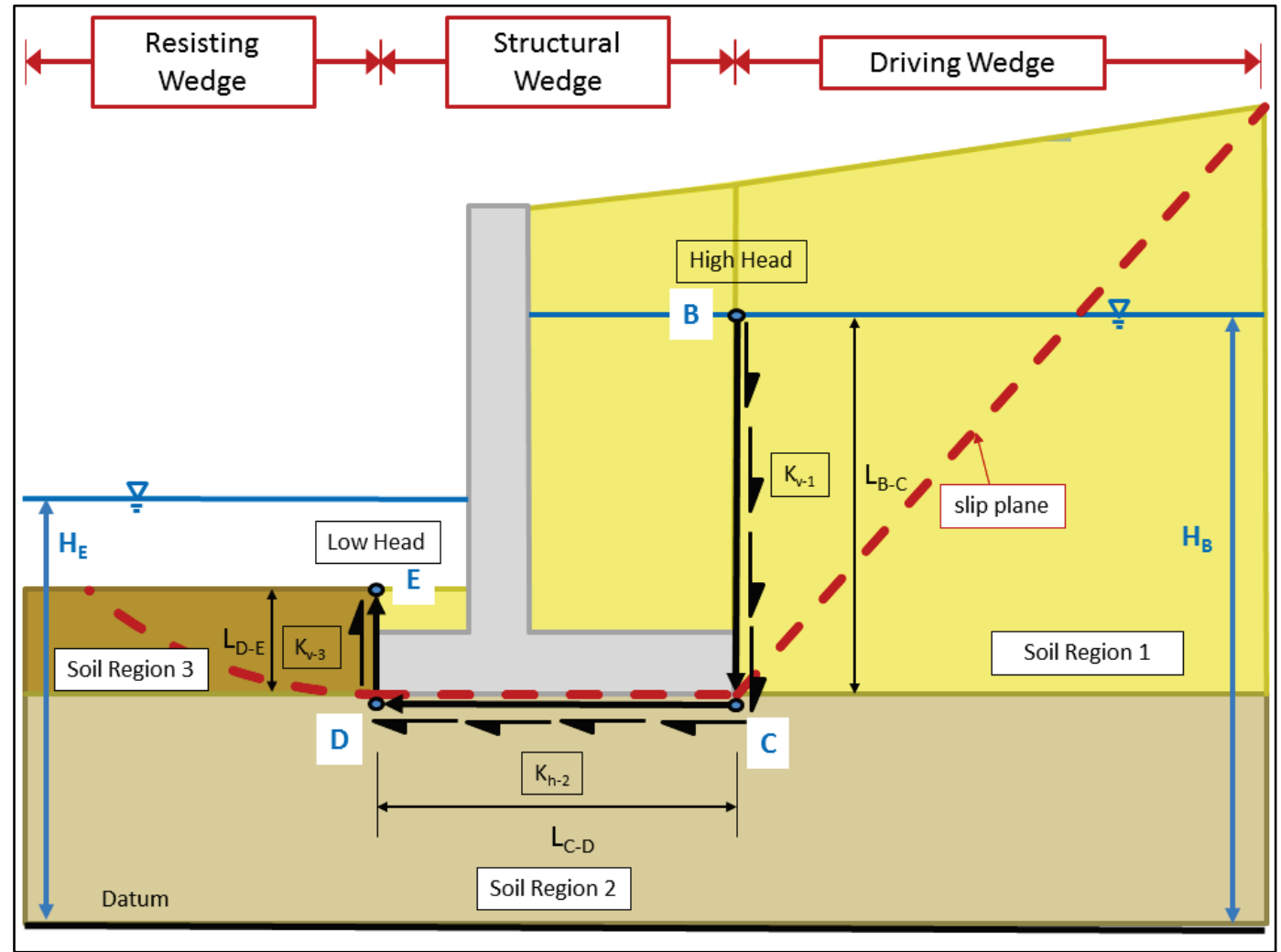


Figure 2-7. 1-D seepage path. (a) Constant slope in total head with distance along the transformed length of line of seepage in a homogenous, 1-D seepage path through all $K_{v-1}$ material. (b) Variation of slope in total head with distance along the three soil regions of the line of seepage.

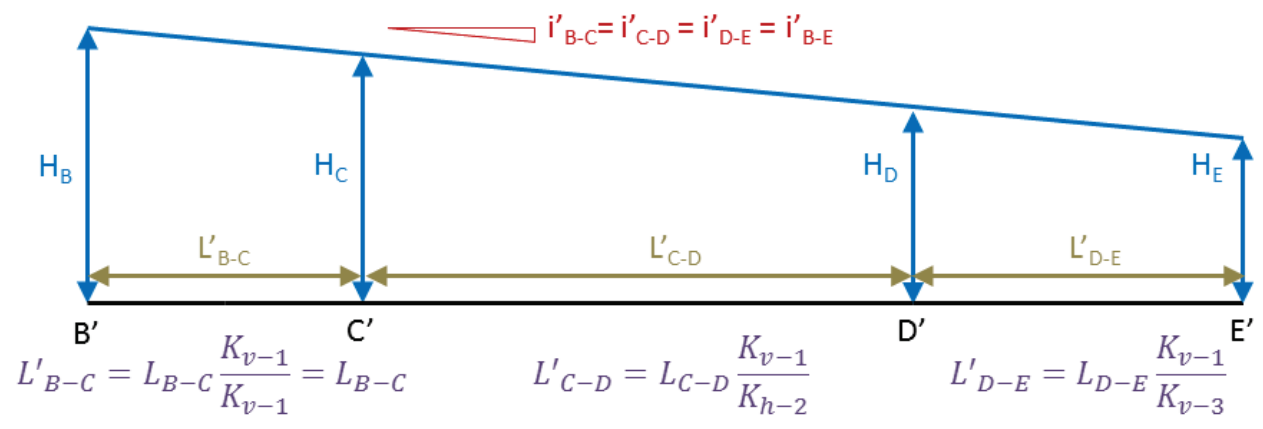

(a)

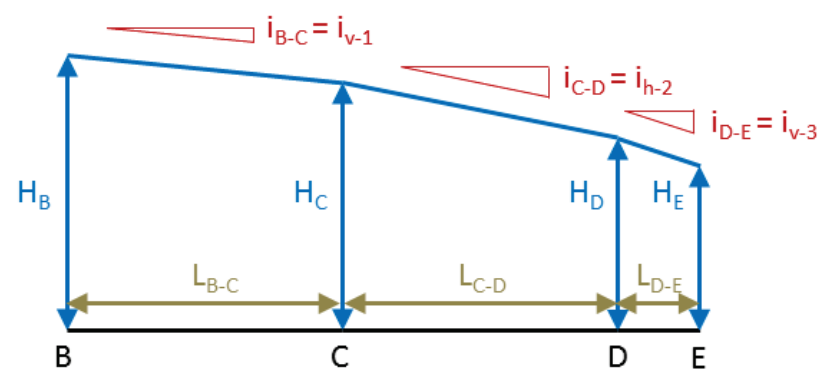

(b)

Observe that the transformed Figure 2-7(a) is required to compute the head values for $H_{C}$ and $H_{D}$ from $H_{B}$ and $H_{E}$ at the transformed position points of B, $C^{\prime}$ and D', respectively. Figure 2-7(b) shows these computed heads of $H_{C}$ and $H_{D}$ and actual heads $H_{B}$ and $H_{E}$ are mapped back to their actual position points $\mathrm{B}, \mathrm{C}, \mathrm{D}$, and $\mathrm{E}$. Here, the hydraulic gradient is constant along the segment of the 1-D flow line contained within each of the three Figure 2-6 soil regions and Figure 2-7(b) segments. The seepage gradients are then computed as equal to the change in total head divided by the actual length of the streamline segment. The actual gradients for each of the three regions are designated as $i_{v-1}, i_{h-2}$ and $i_{v-3}$, respectively, in Figure 2-7(b). $i_{v-1}$ is substituted for $i_{y-D W}$ in Equation 2.9 and $i_{v-3}$ is substituted for $i_{y-R W}$ in Equation 2.19 when computing the equivalent unit weights $\gamma_{e}$ for the Driving soil and Resisting soil Wedges, respectively.

The pressure head $h_{p}$ is computed at any point along each of the three Figure 2-7 streamline segments using Bernoulli's equation for total head $(H)$ and assuming the velocity head is negligible. 


$$
h_{p}=H-h_{e}
$$

where $h_{e}$ is the elevation head for the point.

This modified relationship allows for the computation of the corresponding pore water pressure $(u)$ at the selected point by

$$
u=h_{p} * \gamma_{w}
$$

\subsubsection{Line of Seepage - gap}

There are slight changes for the case in which there is partial separation between the Structural Wedge and its foundation. This may occur when the effective resultant normal force $N^{\prime}$ between the base of the T-Wall and its foundation is outside the kern (i.e., middle third region of the base). The modified Line of Seepage analysis is idealized in Figure 2-8 for this special case. When the distance to the effective normal force $N$ ' as measured from the Toe of the T-Wall, designated $X_{N-t o e}$, is less than one-third the width of the base, a gap will develop starting at the heel point of the base. The length of the gap, $L_{g a p}$, is calculated as

$$
L_{g a p}=\text { Base }-3 * x_{N-t o e}
$$

By the geometry in Figure 2-8, the effective base area in compression, $B_{e}$, is given by

$$
B_{e}=\text { Base }-L_{g a p}
$$

or, equivalently, as

$$
B_{e}=3 * x_{N-t o e}
$$

with the geometric restriction that the value for $B_{e}$ is less than or equal to the base width, Base. 
Figure 2-8. Seepage path for gradient determination assuming base separation along a portion of the base of the Structural Wedge with its foundation, starting at the heel.

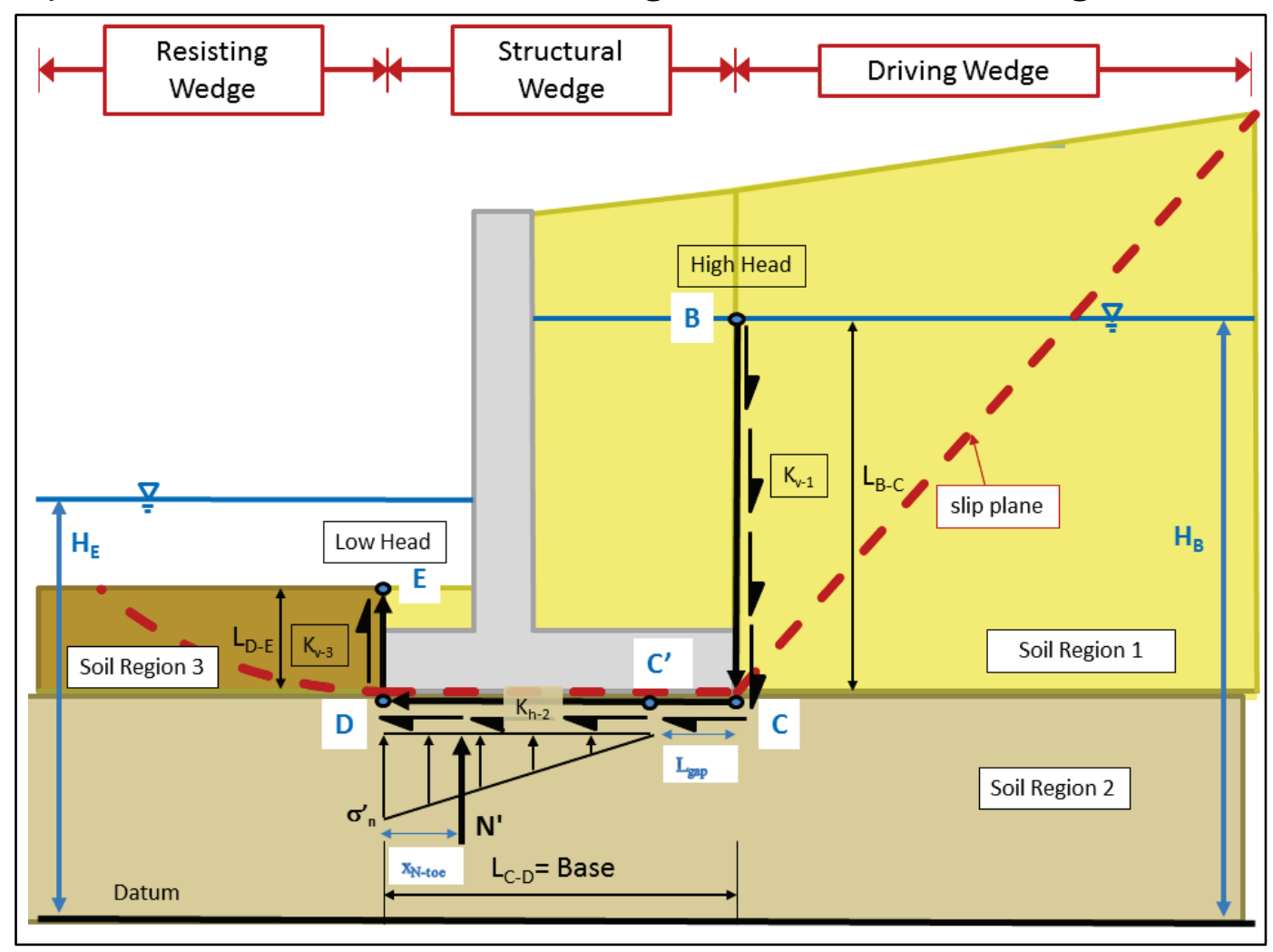

To account for the gap of length $L_{g a p}$ extending from the Toe (point C) to the tip of the compressive base pressure $\sigma_{n}^{\prime}$ distribution (at point $C^{\prime}$ ) in a Line of Seepage analysis, the 1-D line segment C-C' is inserted into the Figure 2-7 1-D seepage path analysis, resulting in Figure 2-9. This path now consists of four segments: B to C, C to C', C' to D, and D to E. For the gap zone, a high hydraulic conductivity value is assigned. The hydraulic conductivity assigned to the horizontal gap segment $\mathrm{C}$ to $\mathrm{C}^{\prime}$ in Figure $2-8$ is $1 \mathrm{~cm} / \mathrm{sec}$. It is three orders of magnitude larger than those listed in Table 2-1. This high hydraulic conductivity value ensures that there will be virtually no total head loss along the Figure 2-8 $L_{g a p}$ length in the 1-D Line of Seepage computation. The total head transformation equation graphically depicted in Figures 2-9(a) and 2-9(b) are applied to all four 1-D segments in the analysis in the usual manor. The transformed segment from point $C$ to $C^{\prime}$ in the Figure 2-9(a) transformed figure should show the same or nearly the same total head values at these two points. Again, virtually no head loss occurs during water seepage within a gap. Equation 2.24 for pressure head $h_{p}$ and Equation 2.25 for pore water pressure are also applied in the usual manor. 
Figure 2-9. 1-D seepage path with base gap. (a) Constant slope in total head with distance along the transformed length of line of seepage in a homogenous, 1-D seepage path through all $\mathrm{K}_{\mathrm{v}-1}$ material. (b) Variation of slope in total head with distance along the three soil regions (and 1 region of gap) of the line of seepage.

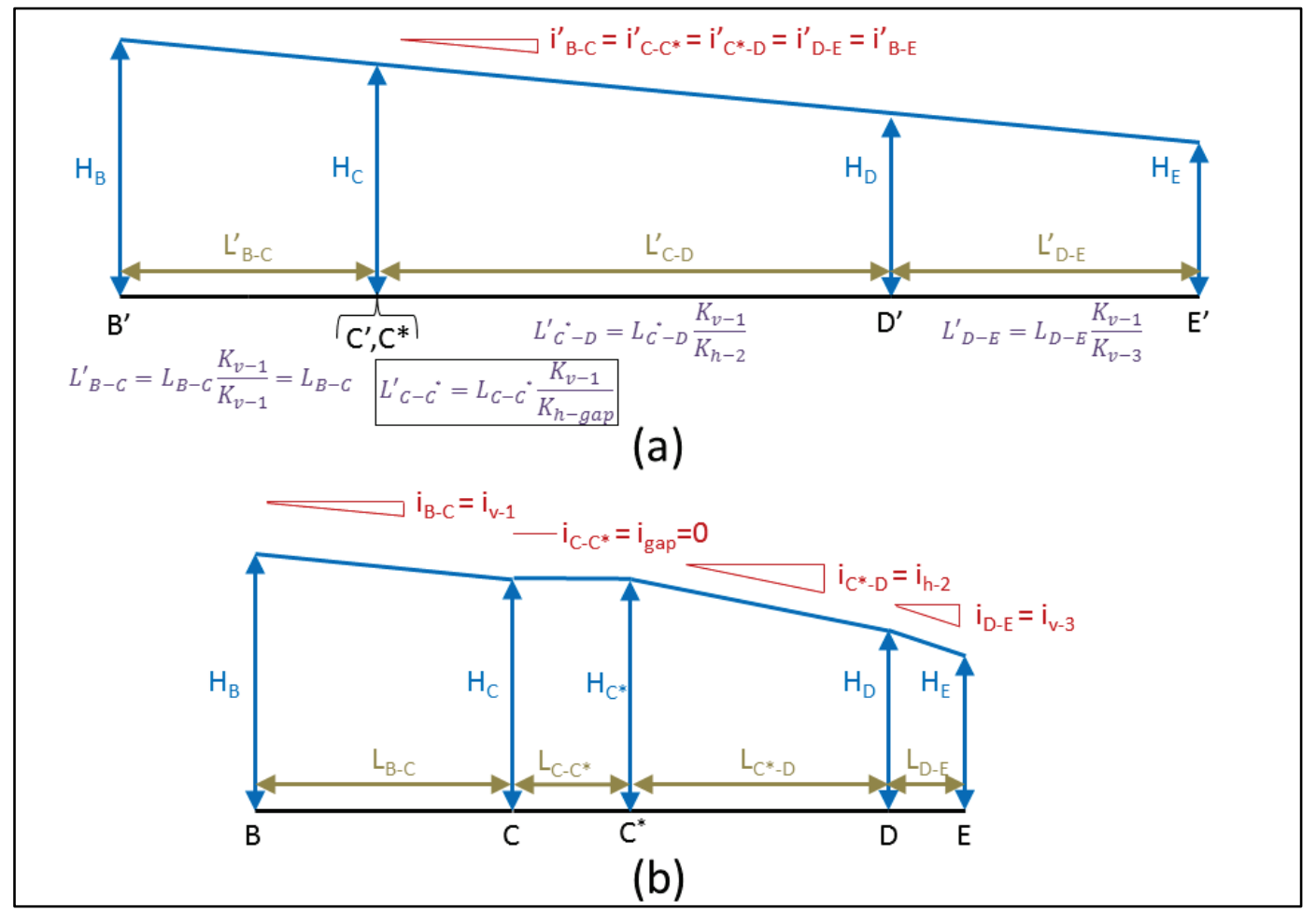

\subsection{Shear force, resultant normal force $N^{\prime}$ and its location along the base of the Structural Wedge}

The forces applied to the Structural Wedge are depicted in Figure 2-10. The Resisting Wedge and Driving Wedge impose forces $P_{L}$ and $P_{R}$, respectively, upon this wedge. 
Figure 2-10. Free body diagram of forces acting on the Structural Wedge, including the left- and right-hand side forces resulting from the Resisting Wedge and Driving Wedge, $P_{L}$ and $P_{R}$, respectively.

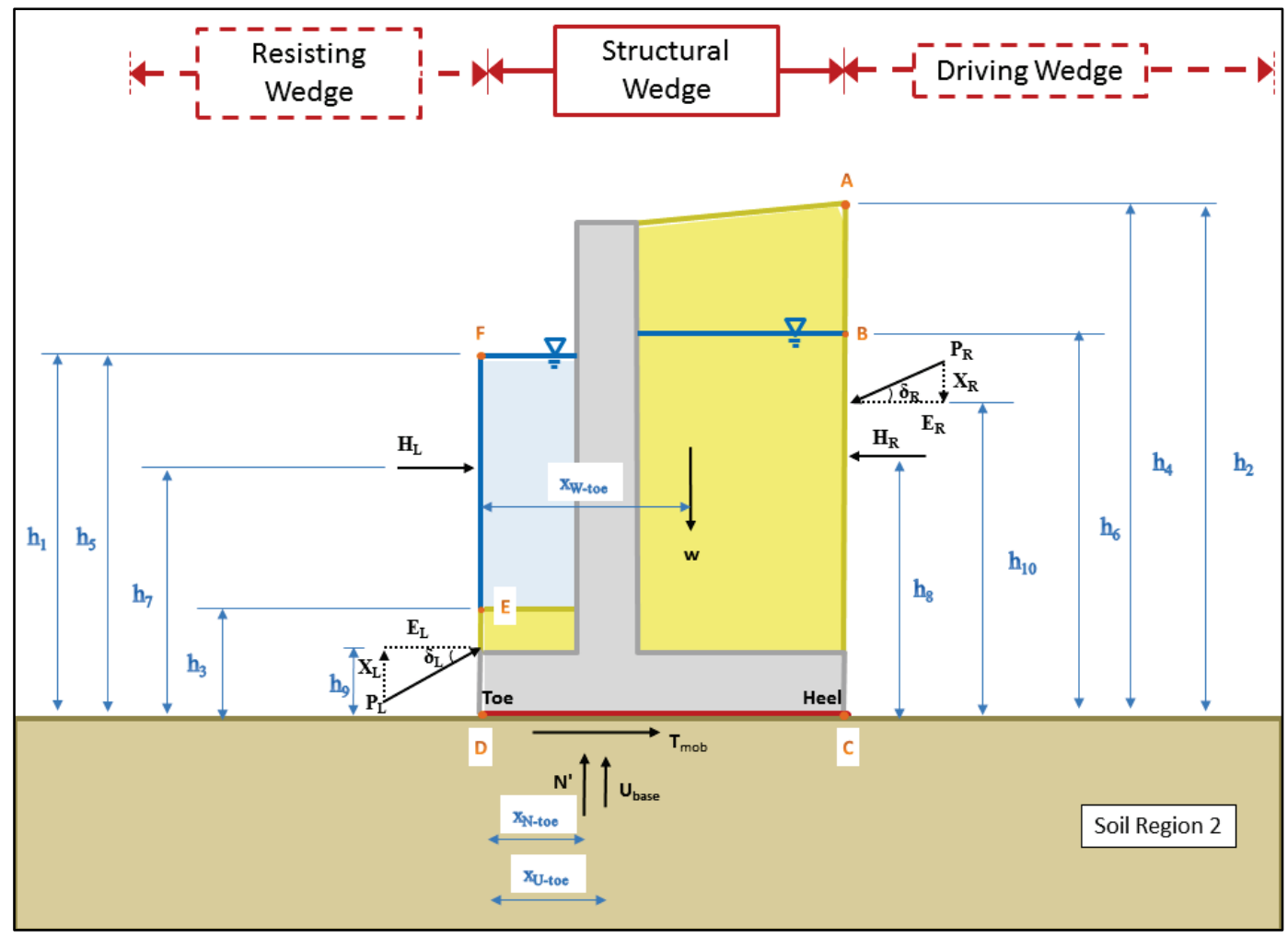

The symbols used in this Structural Wedge are defined as follows:

- $\quad$ Base is the base width of the Structural Wedge.

- $\quad E_{L}$ is the horizontal shear force component of $P_{L}$ acting on the left side of the wedge.

- $\quad E_{R}$ is the horizontal shear force component of $P_{R}$ acting on the right side of the wedge.

- $h_{1}$ is the height from point $\mathrm{D}$ to the topmost point on the left side of the Structural Wedge free body diagram (point F).

- $\quad h_{2}$ is the height from point $\mathrm{C}$ to the topmost point on the right side of the Structural Wedge free body diagram (point A).

- $\quad h_{3}$ is the height from point D to the top of the buttressing soil on the left side of the Structural Wedge (point E).

- $h_{4}$ is the height from point $\mathrm{C}$ to the top of the retained soil on the right side of the Structural Wedge (point A).

- $h_{5}$ is the height from point D to the top of the top of pool on the left side of the Structural Wedge (point F).

- $h_{6}$ is the height from point $\mathrm{C}$ to the top of the top of the piezometric surface on the right side of the Structural Wedge (point B). 
- $h_{7}$ is the height from point $\mathrm{D}$ to $H_{L}$.

- $h_{8}$ is the height from point $\mathrm{C}$ to $H_{R}$.

- $h_{9}$ is the height from point D to $E_{L}$.

- $h_{10}$ is the height from point $\mathrm{C}$ to $E_{R}$.

- $H_{L}$ is the horizontal resultant force exerted by the distribution of pool and pore water pressure acting on the left side of the wedge.

- $H_{R}$ is the horizontal resultant force exerted by the distribution of pore water pressure acting on the right side of the wedge.

- $\quad N^{\prime}$ is the effective force acting normal to the base of the wedge.

- $\quad P_{L}$ is the resultant interslice force acting on the left side of the wedge.

- $P_{R}$ is the resultant interslice force acting on the right side of the wedge.

- $T_{m o b}$ is the mobilized shear resistance force acting along the base of the wedge.

- $U_{\text {base }}$ is the resultant water pressure force acting normal to the base calculated using the average of the pore water pressures at points $\mathrm{C}$ and D. Ubase acts normal to the base of the wedge.

- $W$ is the (total) weight of the wedge.

- $\quad X_{L}$ is the vertical shear force component of $P_{L}$ acting on the left side of the wedge.

- $X_{R}$ is the vertical shear force component of $P_{R}$ acting on the right side of the wedge.

- $x_{N-t o e}$ is the distance from the Toe (point D) to the effective resultant normal force $N$ '.

- $x_{N-b a s e}$ is the distance from the Toe (point D) to the resultant uplift force Ubase acting normal to wedge base.

- $x_{W-t o e}$ is the distance from the Toe (point D) to the weight of the structural wedge $W$.

The Greek character symbols are defined as follows:

- $\alpha$ is the angle the base of the planar wedge as measured from horizontal. $\alpha=0$ deg in this example and is therefore not included in this figure.

- $\delta_{L}$ is the angle of interslice friction on the left side of the wedge.

- $\delta_{R}$ is the angle of interslice friction on the right side of the wedge.

The resultant mobilized active earth pressure force labeled $P_{R}$ in Figure 210 is imposed on the right-hand side of the Structural Wedge by the Driving Wedge. Similarly, the resultant mobilized passive earth pressure force labeled $P_{L}$ in Figure 2-10 is imposed on the left-hand side of the Structural Wedge by the Resisting Wedge. 
The summation of vertical forces acting on the Figure 2-10 T-Wall results in the expression for the effective vertical force normal to the base of the TWall,

$$
N^{\prime}=W-U_{\text {base }}+X_{R}-X_{L}
$$

The summation of horizontal forces acting on the Figure 2-10 T-Wall results in the mobilized shear resistance force acting along the base of the wedge,

$$
T_{m o b}=E_{R}+H_{R}-E_{L}-H_{L}
$$

It is the shear force required for horizontal equilibrium of the Structural Wedge. The ultimate horizontal force is given by

$$
T_{u l t}=c^{\prime} * B_{e}+N^{\prime} \tan \left(\phi^{\prime}\right)
$$

where $c$ ' is the effective cohesion and $\phi^{\prime}$ is the effective angle of internal friction.

The FS at the foundation is then given by

$$
F S_{\text {foundation }}=\frac{T_{\text {ult }}}{T_{\text {mob }}}
$$

Summation of moments about the Toe of the Figure 2-10 T-Wall results in

$$
N^{\prime} * x_{N-\text { toe }}=\left\{\begin{array}{c}
W * x_{W-\text { toe }}-U_{\text {base }} * x_{U-\text { toes }}+E_{L} * h_{3}+H_{L} * h_{1}+X_{R} * \text { Base }- \\
E_{R} * h_{2}-H_{R} * h_{4}
\end{array}\right\}(2
$$

Solving for $x_{N-\text { toe }}$

$$
x_{N-\text { toe }}=\frac{W * x_{W-\text { toe }}-U_{\text {base }} * x_{U-\text { toe }}+E_{L} * h_{3}+H_{L} * h_{1}+X_{R} * \text { Base }-E_{R} * h_{2}-H_{R} * h_{4}}{N^{\prime}}
$$

Recall that for the Normal load case, the EM 1110-2-2502 (HQUSACE 1989) performance criteria stipulates that $B_{e}$ is required to be equal to the base width, Base, but this in a deterministic calculation. When probabilistic engineering soil property criteria are considered through a simulation procedure of analysis, there are situations in which the $B_{e}$ value can be less than Base. However, values of $B_{e}$ less than Base will violate the EM 1110-2- 
2502 (HQUSACE 1989) design criteria for Normal loads. This would be a violation of the base area in compression limit state criteria.

In preparation for a performance function probabilistic simulation assessment, Equation 2.33 needs to be recast. Rearranging, Equation 2.28 becomes

$$
x_{N-t o e}=\frac{B_{e}}{3}
$$

Recall that the value for $B_{e}$ is geometrically restricted to be less than or equal to the base width, Base.

Introducing Equation 2.35, Equation 2.33 is recast as

$$
N^{\prime} *\left(\frac{B_{e}}{3}\right)=\left\{\begin{array}{c}
W * x_{W-\text { toe }}-U_{\text {base }} * x_{U-\text { toe }}+E_{L} * h_{9}+H_{L} * h_{7}+X_{R} * \text { Base }- \\
E_{R} * h_{10}-H_{R} * h_{8}
\end{array}\right\}
$$

where $B_{e}$ is constrained geometrically to be less than or equal to Base. A gap is formed when $B_{e}$ is less than Base, and the length of the gap is determined by subtracting $B_{e}$ from Base.

\subsubsection{Location of Driving Wedge Force $E_{R}$}

Figure 2-11 shows the horizontal component of the effective earth pressure distribution and the distribution of water pressures with their corresponding resultant forces acting on the right side of the Structural Wedge. 
Figure 2-11. Horizontal component of effective earth pressures and water pressures with their corresponding resultant forces acting on the right side of the Structural Wedge.

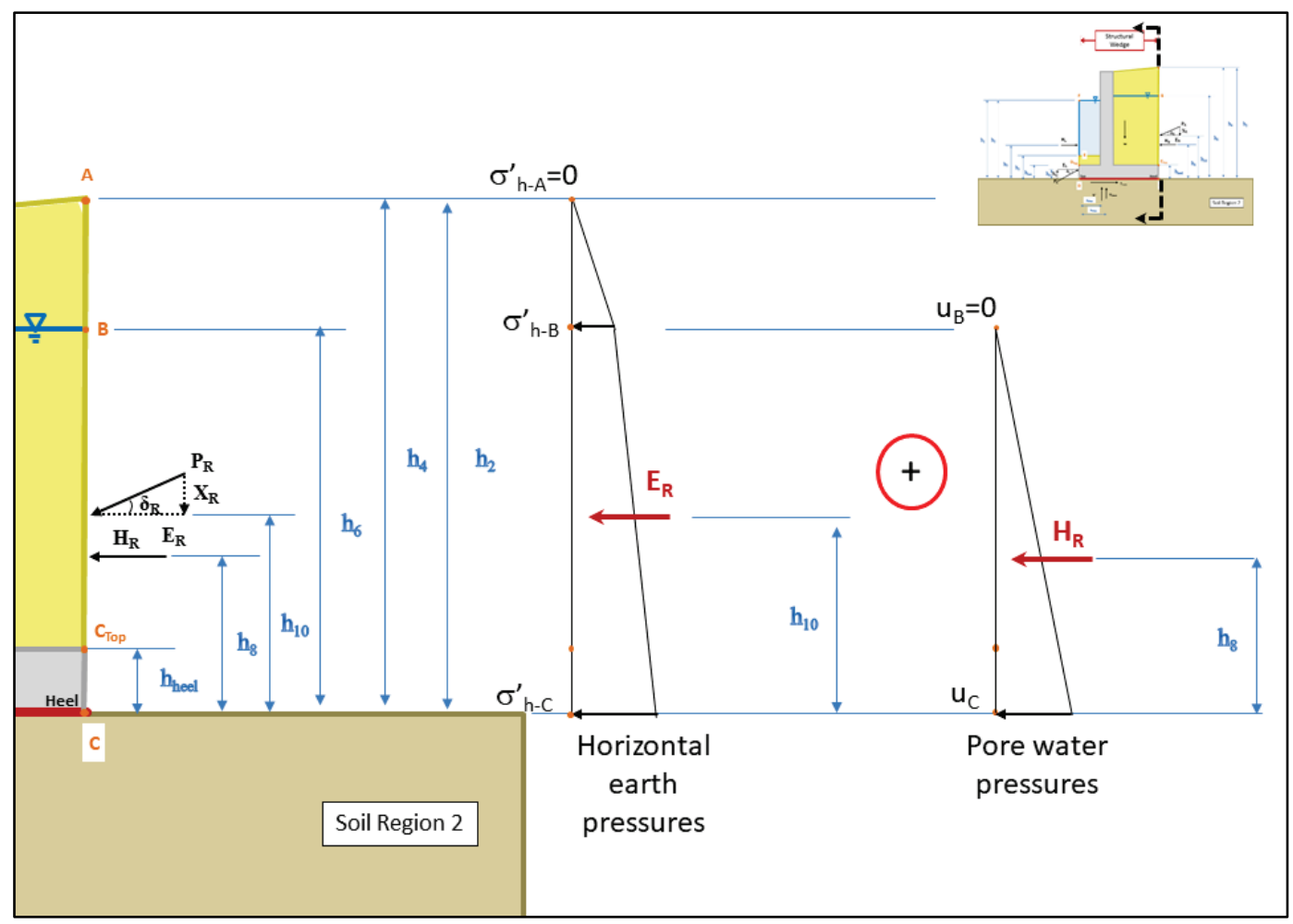

The horizontal effective earth pressure at point B in Figure B-2 is given by

$$
\sigma_{h-B}^{\prime}=K_{\text {Active }} * \cos \left(\delta_{\text {mob-Active }}^{\prime}\right) * \gamma_{\text {moist }} *\left(h_{4}-h_{6}\right)
$$

where

$\gamma /$ moist is the moist unit weight of the retained soil, and $K_{\text {Active }}$ is mobilized active earth pressure coefficient given by Equation 2.4 and is calculated using $\phi_{\text {mob-Active }}^{\prime}$ and $\delta_{\text {mob-Active. }}^{\prime}$

The horizontal effective earth pressure at point $C$ is

$$
\sigma_{h-C}^{\prime}=K_{\text {Active }} * \cos \left(\delta_{\text {mob-Active }}^{\prime}\right) *\left\{\gamma_{\text {moist }} *\left(h_{4}-h_{6}\right)+\gamma_{1} * h_{6}\right\}(2
$$

The point of application of $E_{R}$ is given by

$$
h_{10}=\frac{\operatorname{MomER}_{A-B}+\operatorname{MomER}_{B-C}}{E_{R}}
$$


with

$$
\operatorname{MomER}_{A-B}=\left[\frac{1}{2} *\left(\sigma_{h-B}^{\prime}\right) *\left(h_{4}-h_{6}\right)\right] *\left[\frac{1}{3} *\left(h_{4}-h_{6}\right)+h_{6}\right]
$$

and

$$
\operatorname{MomER}_{B-C}=\left\{\begin{array}{c}
\left\{\left[\left(\sigma_{h-B}^{\prime}\right) *\left(h_{6}\right)\right] *\left[\frac{1}{2} *\left(h_{6}\right)\right]\right\}+ \\
\left\{\left[\frac{1}{2} *\left(\sigma_{h-C}^{\prime}-\sigma_{h-B}^{\prime}\right) *\left(h_{6}\right)\right] *\left[\frac{1}{3} *\left(h_{6}\right)\right]\right\}
\end{array}\right\}
$$

\subsubsection{Location of horizontal water pressure resultant force - right side of the Structural Wedge}

The resultant pore water pressure force $H_{R}$ is given by

$$
H_{R}=\frac{1}{2} *\left(u_{C}\right) *\left(h_{6}-h_{\text {heel }}\right)
$$

Recall that the pore water pressure $u_{c}$ may be computed using the line of seepage (Subsection 2.3.3 or 2.3.4).

The point of application of $H_{R}$ with reference to the elevation of the bottom of the base slab at its heel (i.e., point $\mathrm{C}$ ), is given by

$$
h_{8}=\frac{M o m H R_{B-C}}{H_{R}}
$$

with the moment about the elevation of the bottom of the base of the slab, defined as

$$
\operatorname{MomHR}_{B-C}=\left[\frac{1}{2} *\left(u_{C}\right) *\left(h_{6}\right)\right] *\left[\frac{1}{3} *\left(h_{6}\right)\right]
$$

\subsubsection{Location of Resisting Wedge force $E_{L}$}

Figure 2-12 shows the horizontal component of the effective earth pressure distribution and the distribution of water pressures with their corresponding resultant forces acting on the left side of the Structural Wedge. 
Figure 2-12. Horizontal component of effective earth pressures and water pressures with their corresponding resultant forces acting on the left side of the Structural Wedge.

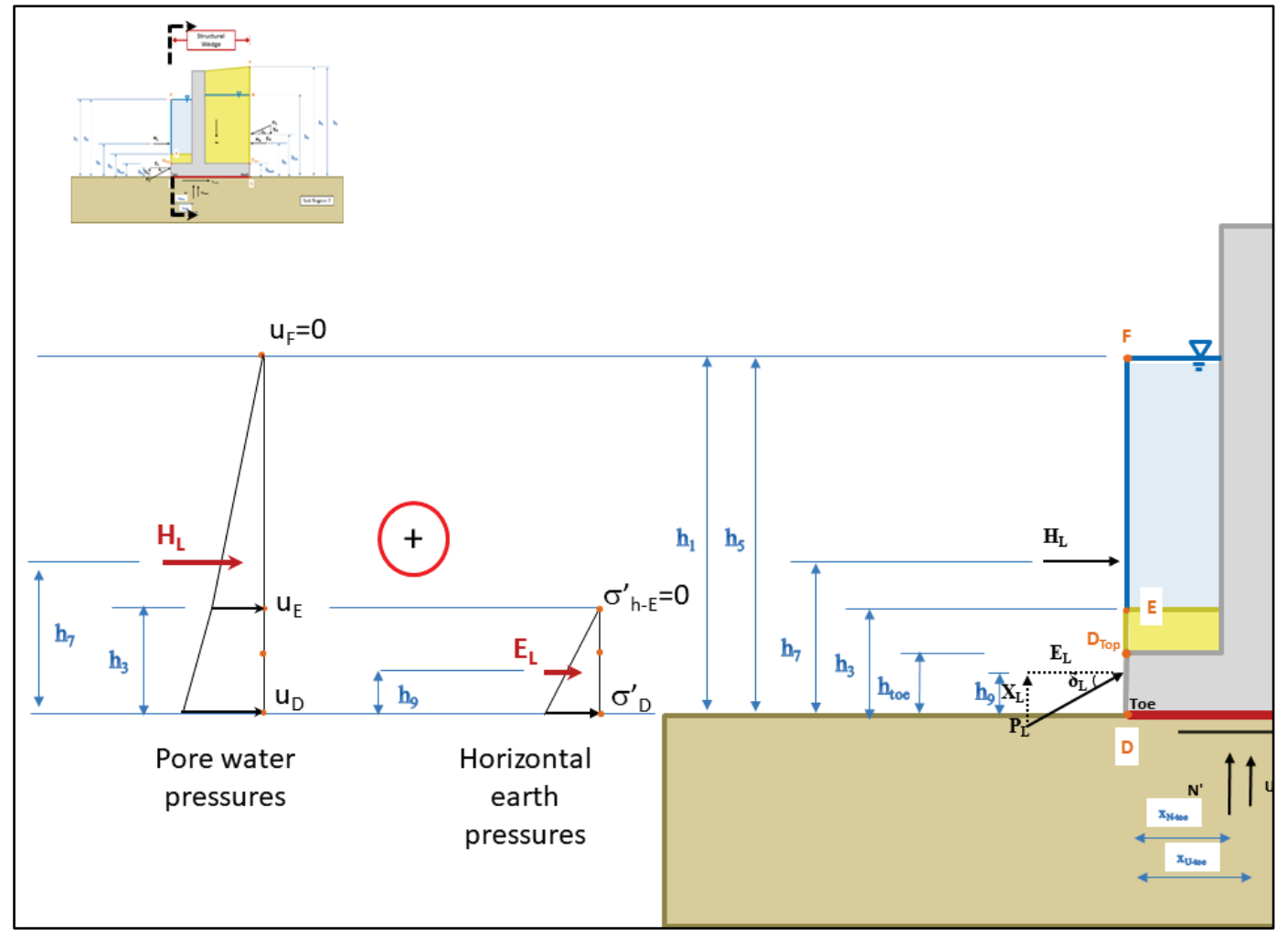

The horizontal effective earth pressure at point D is

$$
\sigma_{h-D}^{\prime}=K_{\text {Active }} * \cos \left(\delta_{\text {mob-Passive }}^{\prime}\right) *\left\{\gamma_{1} * h_{3}\right\}
$$

where

$\gamma_{1}$ is the effective unit weight of the retained soil accounting for the upward, vertical gradient of steady-state seepage through the saturated soil volume of the Driving Wedge. Equation 2.19 provides for the $\gamma_{1}$ value.

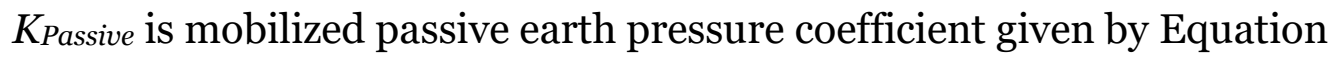
2.16 and is calculated using $\phi^{\prime}$ mob-Active and $\delta_{\text {mob-Active. }}^{\prime}$

The point of application of $E_{L}$, relative to the bottom of the base slab below the toe (point $\mathrm{D}$ ), is given by

$$
h_{9}=\frac{M o m E L_{D-E}}{E_{L}}
$$


with

$$
\operatorname{MomE} L_{D-E}=\left[\frac{1}{2} *\left(\sigma_{h-D}^{\prime}\right) *\left(h_{3}\right)\right] *\left[\frac{1}{3} *\left(h_{3}\right)\right]
$$

The value for height $h_{9}$ by Equation 2.46 should be equal to one-third of $h_{3}$.

\subsubsection{Location of horizontal water pressure resultant force - right side of the Structural Wedge}

The computation of the pore water pressures acting normal to the Structural Wedge using the Line of Seepage procedure of analysis is discussed in Subsection B.2.2. The resultant pore water pressure force $\mathrm{H}_{\mathrm{L}-1}$ is given by

$$
H_{L}=\frac{1}{2} *\left(u_{E}\right) *\left(h_{5}-h_{3}\right)+\frac{1}{2} *\left(u_{E}+u_{D}\right) *\left(h_{3}\right)
$$

The point of application of $H_{L}$, relative to the toe of the base slab (point D), is given by

$$
h_{7}=\frac{M o m H L_{F-E}+M o m H L_{E-D}}{H_{L}}
$$

with

$$
\operatorname{MomHL}_{F-E}=\left[\frac{1}{2} *\left(u_{E}\right) *\left(h_{5}-h_{3}\right)\right] *\left[\frac{1}{3} *\left(h_{5}-h_{3}\right)+h_{3}\right]
$$

and

$$
\operatorname{MomH} L_{E-D}=\left\{\begin{array}{c}
\left\{\left[\left(u_{E}\right) *\left(h_{3}\right)\right] *\left[\frac{1}{2} *\left(h_{3}\right)\right]\right\}+ \\
\left\{\left[\frac{1}{2} *\left(u_{D}-u_{E}\right) *\left(h_{3}\right)\right] *\left[\frac{1}{3} *\left(h_{3}\right)\right]\right\}
\end{array}\right\}
$$

\subsection{Performance function}

As discussed in Subsection 2.4 of Ebeling and White (2019), each limit state boundary is represented mathematically by a performance function that is positive for satisfactory performance of the structure, negative for unsatisfactory, and 0.0 at the transition point of the limit state being investigated. As an example, the performance function can be defined with respect to the capacity of the structure, $R$, and the demand on the structure, $L$. With respect to the capacity and demand, the performance function becomes

$$
g(\boldsymbol{X})=R-L
$$


The Equation 2.52 difference is sometimes referred to as the margin of safety. Using this definition of the performance function, satisfactory performance occurs when the capacity $(R)$ exceeds the demand $(L)$, and unsatisfactory performance occurs when the demand $(L)$ meets or exceeds the capacity $(R)$. The limit state occurs when the demand is balanced by the capacity. This can be related to the probabilities of whether the system performance is satisfactory or unsatisfactory (Figure 2-13). Given that the two variables $R$ and $L$ are each defined by a mean, a variance (equal to the square of the standard deviation), and a distribution (e.g., normal, lognormal), the PUP is the portion of the resulting distribution of $(R-L)$ that falls below zero, as depicted and labeled in Figure 2-13. Since it is a ULS, the PUP is also referred to as probability of failure, $P_{f}$, in the civil engineering literature. Note that since this is a PDF, the area under the $(R$ $L$ ) distribution is equal to 1.0, as are each of the PDF areas under the $R$ and $L$ distributions.

Figure 2-13. Capacity vs. demand related to the probability of unsatisfactory performance (after Nowak and Collins 2000, 2013).

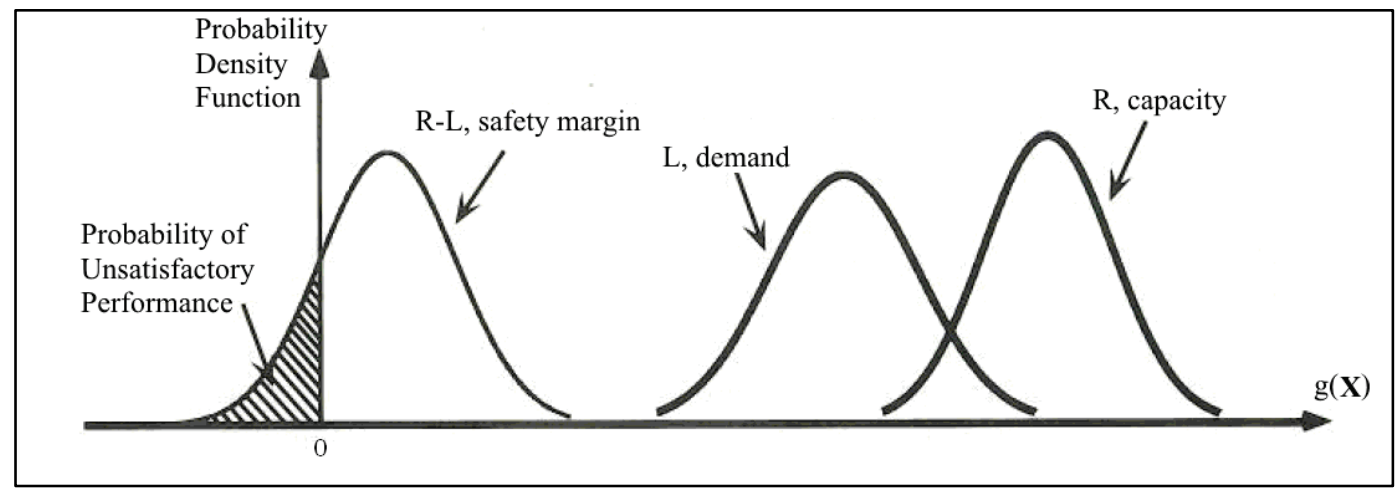

The interrelationship between Reliability theory and Load and Resistance factors used in an LRFD methodology is derived by returning to the simple ULS example of Figure 2-13 and Equation 2.52. The single limit state response surface is defined by setting Equation 2.52 equal to zero,

$$
g(X)=(R-L)=0
$$

where $R$ is the capacity (i.e., $X_{2}=R$ ) and $L$ is demand (i.e., $X_{1}=L$ ). Recall that when $(R-L)<0$, failure occurs. 


\section{The Five Limit States Being Considered for the Example Soil-Structure Interaction of the T-Wall Model with Material Variability}

\subsection{Five limit states}

In general, soil-founded (or rock-founded) reinforced concrete hydraulic structures can possess up to seven limit states. These limit states are summarized in Chapter 1. For the three wedge T-Wall SSI example problem introduced in Chapter 2, five of the seven limit states are being considered in the example problem. These five limit states are

1. Overturning or rotational failure of the structural system about its foundation in its entirety

2. Global sliding failure of the structural system in its entirety

3. Bearing failure of the foundation to the structural system in its entirety

4. Flexural failure of individual structural members

5. Shear failure of individual structural members.

The first three limit states are concerned with geotechnical effects on system response. The latter two limit states are concerned with the reinforced concrete structural features.

\subsection{Initial structural system design considerations}

The full system that will be modeled for these limit states is a T-Wall as shown in Figure 2-1. The primary design feature for this structure that will determine satisfactory or unsatisfactory performance is the base width of the T-Wall. To perform a preliminary deterministic design, assign mean values from Table 2-1 for all shear strength variables of a T-Wall under normal loadings (as defined by EM 1110-2-2502 [HQUSACE 1989]) to obtain an initial estimate of the minimum required base width of the Chapter 2 example T-Wall. This preliminary deterministic design is facilitated by using the CASE software CTWall-R. CTWall-R is an updated version of CTWall (Pace 1994). Note that CTWall-R makes limiting assumptions in its wedge solution scheme so its results will be different from that of the solution made using the engineering relationships given in Chapter 2 of this report. This analysis is used solely to generate an initial, approximate estimate for the minimum T-Wall base width value, Base. 
Once the design of the T-Wall structural system has been defined, the weight of the Structural Wedge can be determined from geometry, soil properties, and water levels. Note that total soil unit weight(s) (e.g., $\gamma_{s a t}$ below the water table) are used to compute $W$ in this effective stress analysis.

These two initial Structural Wedge properties of Base and $W$ are used as constants in the following limit state iterative procedural methods.

\subsection{Base area in compression of the Structural Wedge limit state}

In Subsection 3.1, the first limit state deals with overturning or rotational failure of the structural system about its foundation in its entirety. In the USACE design process for hydraulic structures, this limit state is assessed through control of the amount of base area in compression for the Structural Wedge. This subsection summarizes the required steps: (1) a deterministic analysis to convert $C O V_{\text {point estimates }}$ to $C O V_{\text {spatial }}$ for the effective angle of internal soil friction of the retained soil of the active wedge and for the buttressing soil of the Resisting Wedge, (2) a procedural method to compute the performance of a structural system for the base area in compression limit state.

\subsubsection{Iterative method for determining interactions between Driving, Resisting, and Structural Wedges for at-rest equilibrium conditions for Base Area in Compression limit state}

Table 3-1 outlines an iterative procedure used to determine the forces and their point of application between the wedges (as shown in Figure 2-3) under at-rest conditions, enforced by steps 4 and 5 . Because the system is defined in moment equilibrium and peak moments are defined by at-rest conditions, a solution that iterates through Driving and Resisting wedge slip-plane angles and foundation gap formation must be iterated through to determine the equilibrium state of the model. This iterative procedure returns (1) the forces and their point of application, (2) the length of the Driving and Resisting Wedge slip planes, (3) line of seepage information, and (4) the effective structural base width $B_{e}$ taking the effect of gap formation due to uplift and interaction moments. 
Table 3-1. Iterative at-rest procedure to calculate Soil Wedge interactions.

\begin{tabular}{|c|c|c|}
\hline Step & $\begin{array}{l}\text { Driving, } \\
\text { Resisting } \\
\text { or } \\
\text { Structural } \\
\text { Wedge(s) }\end{array}$ & Description \\
\hline 1 & $\mathrm{D}, \mathrm{R}$ & $\begin{array}{l}\text { Assign at-rest earth pressures: Assign a FS equal to } 1.5 \text { to the Driving } \\
\text { Wedge and the Resisting Wedge so as to obtain at-rest earth pressures } \\
\text { according to EM 1110-2-2502 (HQUSACE 1989) and their resulting } \\
\text { forces } P_{R} \text { and } P_{L} \text {, respectively, acting on either side of the Structural } \\
\text { Wedge. }\end{array}$ \\
\hline 2 & $\mathrm{D}, \mathrm{R}$ & $\begin{array}{l}\text { Assign soil properties } \phi^{\prime} \text { and } \delta^{\prime} \text { : Assign the appropriate soil properties } \phi^{\prime} \\
\text { and } \delta \text { for the procedure being followed. For a deterministic solution, } \\
\text { apply mean values. For simulations, apply the current simulation run } \\
\text { values } X \text {. }\end{array}$ \\
\hline 3 & $\mathrm{D}, \mathrm{S}, \mathrm{R}$ & $\begin{array}{l}\text { Line of Seepage: Perform a Line of Seepage analysis using the geometry } \\
\text { determined during the preliminary design with mean hydraulic } \\
\text { conductivity values for the three soil regions. Assume no gap develops } \\
\text { under Normal loading in the initial analysis. Two sets of results are } \\
\text { provided by this analysis: (a) the vertical seepage gradients acting within } \\
\text { the Driving and Resisting Wedges (iy-Dw and } i_{y-R w} \text {, respectively) and (b) } \\
\text { distributions of total heads, } H \text {, pressure heads } h_{p} \text {, and pore water } \\
\text { pressures, } u \text {, along the three faces of the wetted perimeter for the } \\
\text { Structural Wedge. }\end{array}$ \\
\hline 4 & D & 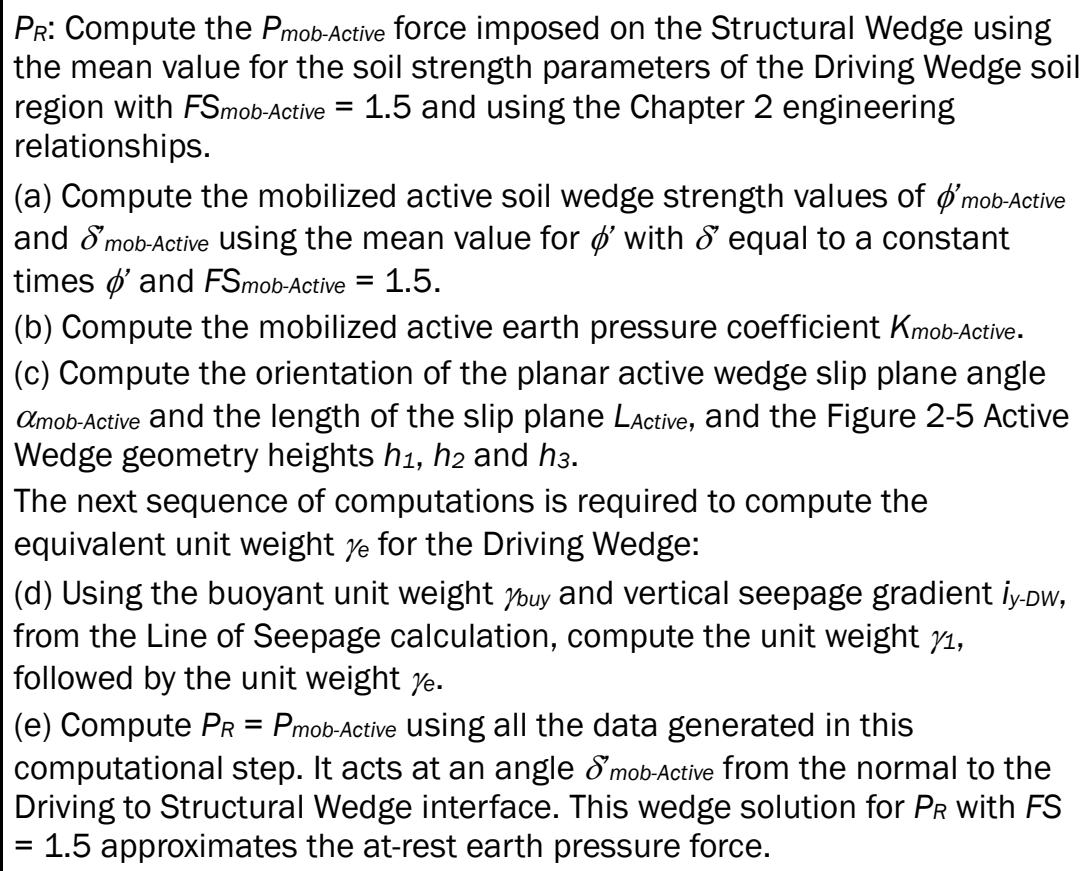 \\
\hline
\end{tabular}




\begin{tabular}{|c|c|c|}
\hline Step & $\begin{array}{l}\text { Driving, } \\
\text { Resisting } \\
\text { or } \\
\text { Structural } \\
\text { Wedge(s) }\end{array}$ & Description \\
\hline 5 & $\mathrm{R}$ & 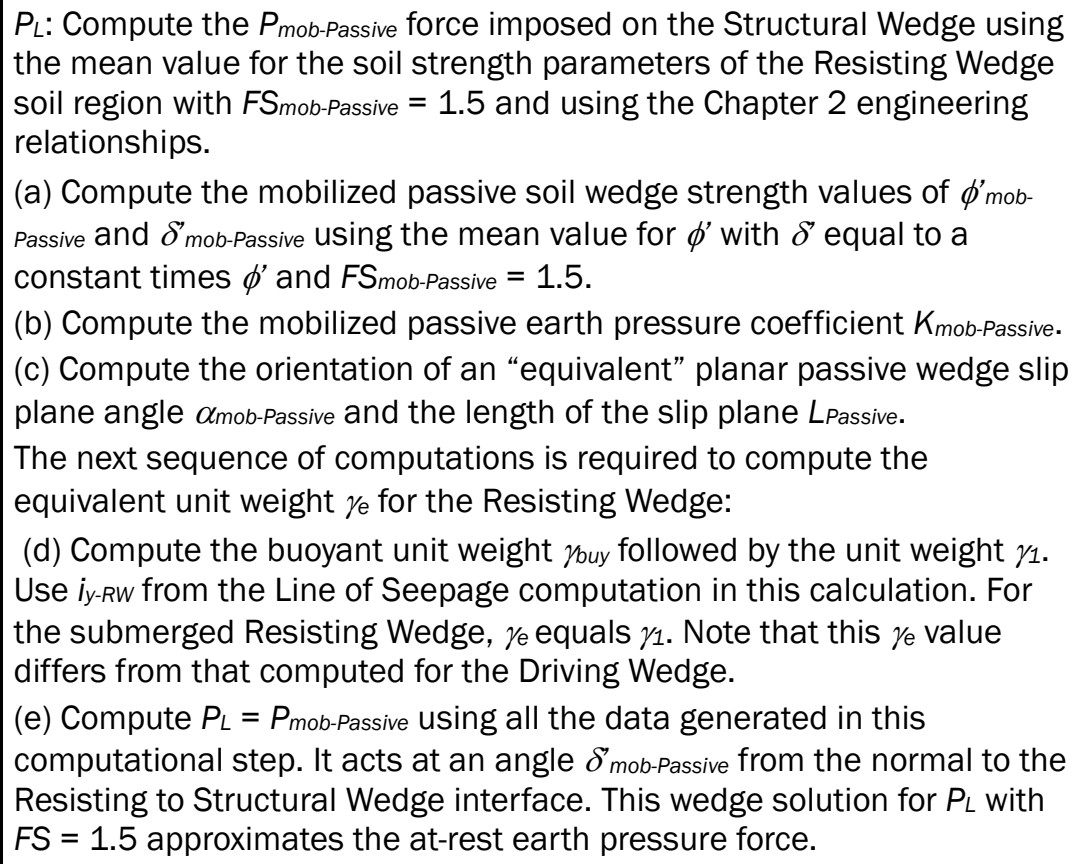 \\
\hline 6 & S & $\begin{array}{l}\text { Boundary Water pressures, } u \text { : Using the results from the Line of Seepage } \\
\text { analysis: (a) compute the distribution of boundary water pressures acting } \\
\text { normal to the left, base and side faces of the structural wedge. } \\
\text { (b) Using this distribution of } u \text {, compute the resultant boundary water } \\
\text { pressure forces acting normal to the three faces of the Structural Wedge: } \\
H_{L}, U_{\text {base, }} \text { and } H_{R} \text {. }\end{array}$ \\
\hline 7 & S & $\begin{array}{l}\text { Check for gap: } \\
\text { (a) Use the vertical force equilibrium Equation (2.29) given in Chapter } 2 \\
\text { to solve for the magnitude of the effective normal force } N^{\prime} \text { acting normal } \\
\text { to the base of the Structural Wedge. } \\
\text { (b) Solve for the location of } N^{\prime} \text { as measured from the Toe of the } \\
\text { Structural Wedge, } X_{N-\text { base. }} \\
\text { (c) Compute the length of gap } L_{g a p} \text {, (if any). }\end{array}$ \\
\hline 8 & $\mathrm{D}, \mathrm{S}, \mathrm{R}$ & $\begin{array}{l}\text { If a gap is computed in the previous step, repeat the analysis starting } \\
\text { with the Line of Seepage analysis and include this gap length: If the } \\
\text { previous step results in a nonzero gap length, repeat steps (3) through } \\
\text { (7) until convergence or a minimum tolerance in } L_{\text {gap }} \text { is met. }\end{array}$ \\
\hline
\end{tabular}

\subsubsection{Engineering steps in the calculation of COVspatial for the Driving and Resisting Wedges and Foundation Soil}

The normal load case (EM 1110-2-2502) (HQSUACE 1989) of the T-Wall design for the base area in compression limit state centers on the application of at-rest earth pressures by the Resisting Wedge and Driving 
Wedge (when present) against the Structural Wedge. The lengths of the slip planes of the Resisting, Driving, and Structural wedges are necessary to determine the geospatial coefficient of variation for Reliability analysis from the point estimate coefficient of variation. The calculation steps of this $C O V_{\text {spatial }}$ for the three soil wedges is described in Table 3-2. The step-bystep base area in compression limit state procedure of analysis of the Structural Wedge is summarized in Table 3-1, as well as the method to determine the slip planes for the wedges. These calculations make use of mean values for $\phi^{\prime}$ and $\delta$ in each of the three soil regions in Table 3-2 to compute the length of the Driving Wedge slip plane $L_{\text {Active }}$ and the length of the Resisting Wedge slip plane LPassive.

Table 3-2 shows the procedure to calculate the coefficient of variation for the soil properties of the three soil wedges of Figure 2-1.

Table 3-2. Procedural method to calculate COV spatial for the Driving and Resisting Wedges.

\begin{tabular}{|c|c|c|}
\hline Step & $\begin{array}{l}\text { Driving, } \\
\text { Resisting or } \\
\text { Structural } \\
\text { Wedge(s) }\end{array}$ & Description \\
\hline 1 & $\mathrm{D}, \mathrm{S}, \mathrm{R}$ & $\begin{array}{l}\text { Determine soil wedge interactions: iterate through Table 3-1, } \\
\text { assigning mean values to } \phi^{\prime} \text { and } \delta^{\prime} \text { for each soil region. }\end{array}$ \\
\hline 2 & D & $\begin{array}{l}\Gamma^{2}(L) \text { Driving Wedge: Compute the Variance Reduction Factor for the } \\
\text { retained soil. } \\
\text { (a) Using the vertical and horizontal scales of fluctuation }(\delta \text { and } \delta \text { ) } \\
\text { for the retained soil (Table } 2-2 \text { ), compute the equivalent scale of } \\
\text { fluctuation } \delta \text { E along the planar slip plane of length } L=L_{\text {Active }} \text { at angle } \\
\alpha_{\text {mob-Active }} \text { (Equation } 2.3 \text { ). } \\
\text { (b) Using Equation } 2.1 \text { with } L=L_{\text {Active, }} \text { compute the Variance } \\
\text { Reduction Factor for the retained soil. }\end{array}$ \\
\hline 3 & D & $\begin{array}{l}C O V_{\text {spatial }} \text { Driving Wedge: Compute } \mathrm{COV}_{\text {spatial }} \text { using Equation } 2.2 \text { of the } \\
\text { effective angle of internal friction for the active wedge using the } \\
\mathrm{COV}_{\text {point estimate value given in Table } 2-1 .}\end{array}$ \\
\hline 4 & $\mathrm{R}$ & 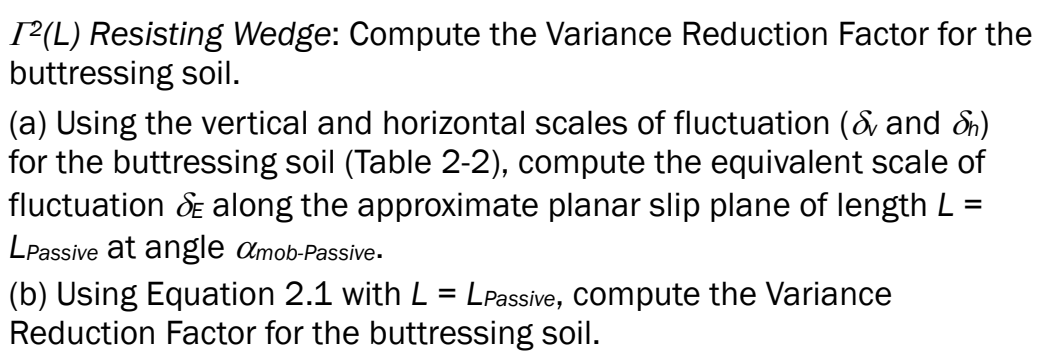 \\
\hline 5 & $\mathrm{R}$ & $\begin{array}{l}C O V_{\text {spatial }} \text { Resisting Wedge: Compute } C_{\text {spatial }} \text { using Equation } 2.2 \text { of } \\
\text { the effective angle of internal friction for the passive wedge using the } \\
\operatorname{COV}_{\text {point estimate value given in Table } 2-1 .}\end{array}$ \\
\hline
\end{tabular}




\begin{tabular}{|l|l|l|}
\hline Step & $\begin{array}{l}\text { Driving, } \\
\text { Resisting or } \\
\text { Structural } \\
\text { Wedge(s) }\end{array}$ & Description \\
\hline 6 & $S$ & $\begin{array}{l}\Gamma \text { (L) Structural Wedge: Compute the Variance Reduction Factor for } \\
\text { the foundation soil. } \\
\text { (a) Set the equivalent scale of fluctuation to the horizontal scale of } \\
\text { fluctuation for the foundation soil }\left(\delta_{E}=\delta_{n}\right) . \text { The example assumes a } \\
\text { horizontal base. } \\
\text { (b) Using Equation 2.1 with L=Base, compute the Variance Reduction } \\
\text { Factor for the foundation soil. }\end{array}$ \\
\hline 7 & $S$ & $\begin{array}{l}\text { COV } \text { spatial Structural Wedge: Compute } C O V_{\text {spatial }} \text { using Equation } 2.2 \text { of } \\
\text { the effective angle of internal friction for the foundation soil using the } \\
C_{\text {COV }} \text { point estimate value given in Table 2-1. }\end{array}$ \\
\hline
\end{tabular}

\subsubsection{Engineering steps to assess the performance (satisfactory/unsatisfactory) of the Structural Wedge for the base area in compression limit state}

For a Reliability analysis of the base area in compression limit state of the Figure 2-3 Structural Wedge, variability in the soil shear strength parameters are considered. Table 2-1 lists the mean and point estimate $\mathrm{COV}$ values for the effective angle of internal friction and interface friction for the three soil regions of the Figure 2-1 T-Wall example problem model. Performing the series of computations outlined in Subsection 3.3.2 results in the transformation of these Table 2-1 point estimate COV values into spatial COV values for each soil region, as required for use in Geotechnical limit state analyses (Ebeling and White 2019). Table 3-3 establishes the steps to compute the performance of a designed T-Wall given a vector of random variables $(\boldsymbol{X})$. For reliability purposes, this function is called the $g(\boldsymbol{X})$ function, as will be discussed in Chapter 4. For the sample T-Wall example, this vector is comprised of the $\phi^{\prime}$ and $\delta$ values for the soil properties in the Driving, Resisting, and beneath the Structural Wedges. For a reliability analysis, a set of vectors will be created, and this function will be used with each of these vectors to determine PUP. In simulation methods, the vector $\boldsymbol{X}$ is created by drawing the values of $\phi$ ' and $\delta$ from their appropriate distributions. 
Table 3-3. Base area in compression limit state procedure of Reliability analysis computing $g(X)$ per simulation value $X$.

\begin{tabular}{|l|l|l|}
\hline Step & $\begin{array}{l}\text { Driving, } \\
\text { Resisting or } \\
\text { Structural } \\
\text { Wedge(s) }\end{array}$ & Description \\
\hline 1 & D, S, R & $\begin{array}{l}\text { Determine soil wedge interactions: iterate through Table 3-1, } \\
\text { assigning values to } \phi^{\prime} \text { and } \delta^{\prime} \text { from } X \text { for each soil region. }\end{array}$ \\
\hline 2 & D, S, R & $\begin{array}{l}\text { Resisting moment } R \text { : Compute the Resisting moment } R \text { for simulation } \\
i \text { using Equation 3.1. }\end{array}$ \\
\hline 3 & D, S, R & $\begin{array}{l}\text { Driving moment } L: \text { Compute the Driving moment } L \text { for simulation } i \\
\text { using Equation 3.2. }\end{array}$ \\
\hline 4 & D, S, R & $\begin{array}{l}\text { Assessing } g(X) \text { : Compute the value of the performance function } g(X) \\
\text { for this simulation } i \text { using Equation 2.52. Note that if } g(X) \text { is positive } \\
\text { then the performance of the structure is counted as satisfactory } \\
\text { against the base area in compression limit state for variable vector } X \text {. } \\
\text { However, if } g(X) \text { is negative then the performance of the structure is } \\
\text { counted as unsatisfactory against the base area in compression limit } \\
\text { state for variable vector } X \text {. }\end{array}$ \\
\hline
\end{tabular}

The Equation 2.36 Resisting moments of Figure 2-10 are combined into a single moment term and the Driving moments of Equation 2.36 are combined into a second term for the Reliability analysis. The Equation 2.36 resisting moments, designated as $\mathrm{R}$, are given as

$$
R=W * x_{W-\text { toe }}+E_{L} * h_{9}+H_{L} * h_{7}+X_{R} * \text { Base }
$$

In the probabilistic simulation process discussed in the next chapter, the moments due to forces $W$, and $H_{L}$ are deterministic. The other two moments due to forces $E_{L}$ and $X_{R}$ are variable and will change in value during each simulation.

The Equation 2.36 driving moments, designated as $L$, are

$$
L=U_{\text {base }} * x_{U-\text { toe }}+E_{R} * h_{10}+H_{R} * h_{8}
$$

Observe that the moment due to force $N$ ' is not included because when the Structural Wedge is verged on moment equilibrium, its point of action is located at the heel. In the probabilistic simulation process discussed in the next chapter, the moment due to force $H_{R}$ is deterministic as well as that due to force $U_{b a s e}$, so long as $B_{e}=$ Base. The other two moments due to forces $N^{\prime}$ and $E_{R}$ are variable and will change in value during each simulation. 
Note that the mobilized values for $\phi^{\prime}$ and $\delta$ are being used in conjunction with this $P_{R}$ value to compute the $E_{R}$ and $X_{R}$ forces the Driving Wedge imposes on the Structural Wedge and not their ultimate strength values. The horizontal force component of mobilized force $P_{R}$ is given as

$$
E_{R}=P_{R} * \cos \left(\delta^{\prime}{ }_{\text {mob-Active }}\right)
$$

The vertical component of the mobilized right-hand side driving (wedge) force is given by

$$
X_{R}=P_{R} * \sin \left(\delta^{\prime}{ }_{m o b-A c t i v e}\right)
$$

Similarly, the mobilized values for $\phi^{\prime}$ and $\delta$ are being used in conjunction with this $P_{L}$ value to compute the $E_{L}$ and $X_{L}$ forces the Resisting Wedge imposes on the Structural Wedge and not their ultimate strength values. The horizontal force component of mobilized force $P_{L}$ is given as

$$
E_{L}=P_{L} * \cos \left(\delta^{\prime}{ }_{\text {mob-Passive }}\right)
$$

The vertical component of the mobilized left-hand side resisting (wedge) force is given by

$$
X_{L}=P_{L} * \sin \left(\delta^{\prime}{ }_{\text {mob-Passive }}\right)
$$

Recall from Chapter 2 that the summation of vertical forces acting on the Figure 2-10 T-Wall results in the expression for the effective vertical force normal to the base of the T-Wall,

$$
N^{\prime}=W-U_{\text {base }}+X_{R}-X_{L}
$$

With $E_{L}$ and $X_{R}$, now defined, the resultant resisting moment $R$ may be computed using Equation 3.1 in Step 2 of Table 3-3. And with $E_{R}, X_{L}$, and $N$ ' established, the resultant driving moment $L$ may be computed using Equation 3.2 in Step 3 of Table 3-3.

The value of the performance function $g(\boldsymbol{X})$ for simulation $i$ is computed in Step 3 using Equation 2.52. Table 3-3 is repeated for simulations $i+1$ through $n$ simulations with the current simulation $\boldsymbol{X}$ values. 
The step-by-step engineering computations, used in the base area in the probabilistic compression limit state procedure of Reliability analysis of the Structural Wedge, are summarized in Table 3-2.

\subsection{Global sliding failure of the Driving, Structural, and Resisting Wedge limit state}

In Subsection 3.1, the second limit state deals with global sliding failure of the structural system in its entirety. This equates to a limit state of the Driving, Structural and Resisting Wedges sliding in unison. In the USACE design process for hydraulic structures, this limit state is assessed through control of the amount of shear resistance mobilized within the soils comprising the Driving, Wedge, foundation soil, and the soil comprising the Resisting Wedge. The USACE guidance document EM 1110-2-2502 (HQUSACE 1989) expresses the required minimum factor of safety, FS, value through a Shear Mobilization Factor (SMF). The SMF is defined as the inverse of the minimum FS value. This subsection summarizes the required steps: (1) a deterministic analysis to convert $C O V_{\text {point estimates }}$ to $C O V_{\text {spatial }}$ for the effective angle of internal soil friction of the retained soil of the active wedge and for the buttressing soil of the Resisting Wedge and (2) a procedural method to compute the performance of a structural system for the global sliding failure limit state. By EM 1110-2502 guidance, the minimum FS value for any Load case (i.e., Usual, Unusual, or Extreme) is specified to be the same value within the Driving, Structural, and Resisting Wedges.

\subsubsection{Iterative method for determining interactions between Driving, Resisting, and Structural Wedges for force equilibrium conditions for Sliding limit state}

Table 3-4 outlines an iterative procedure used to determine the forces and their point of application between the wedges (as shown in Figure 2-3) in equilibrium altering the $F S$, enforced by steps 4 and 5 . Because the system is not defined in force equilibrium, a solution that iterates through Driving and Resisting wedge slip-plane angles and foundation gap formation must be iterated through to determine the equilibrium state of the model, where the factors of safety of the three soil wedges are the same. This iterative procedure returns (1) the forces and their point of application, (2) the length of the Driving and Resisting Wedge slip planes, (3) line of seepage information, and (4) the effective structural base width $B_{e}$ taking the effect of gap formation due to uplift and interaction moments. 
Table 3-4. Iterative procedure to calculate equilibrium force Soil Wedge interactions.

\begin{tabular}{|c|c|c|}
\hline Step & $\begin{array}{l}\text { Driving, } \\
\text { Resisting or } \\
\text { Structural } \\
\text { Wedge(s) }\end{array}$ & Description \\
\hline 1 & $\mathrm{D}, \mathrm{R}$ & $\begin{array}{l}\text { Assign at-rest earth pressures: Assign an initial FS equal to } 1.5 \text { to the } \\
\text { Driving Wedge and the Resisting Wedge to obtain at-rest earth } \\
\text { pressures according to EM 1110-2-2502 (HQUSACE 1989) and their } \\
\text { resulting forces } P_{R} \text { and } P_{L} \text {, respectively, acting on either side of the } \\
\text { Structural Wedge. }\end{array}$ \\
\hline 2 & $\mathrm{D}, \mathrm{R}$ & $\begin{array}{l}\text { Assign soil properties } \phi^{\prime} \text { and } \delta^{\prime} \text { : Assign the appropriate soil properties } \\
\phi^{\prime} \text { and } \delta^{\prime} \text { for the procedure being followed. For a deterministic } \\
\text { solution, apply mean values. For simulations, apply the current } \\
\text { simulation run values } X \text {. }\end{array}$ \\
\hline 3 & $D, S, R$ & $\begin{array}{l}\text { Line of Seepage: Perform a Line of Seepage analysis using the } \\
\text { geometry determined during the preliminary design with mean } \\
\text { hydraulic conductivity values for the three soil regions. Assume no gap } \\
\text { develops under Normal loading in the initial analysis. Two sets of } \\
\text { results are provided by this analysis: (a) the vertical seepage } \\
\text { gradients acting within the Driving and Resisting Wedges ( } i_{y-D w} \text { and } i_{y-} \\
R w, \text { respectively) and distributions of (b) total heads, } H \text {, pressure } \\
\text { heads } h_{p} \text {, and pore water pressures, } u \text {, along the three faces of the } \\
\text { wetted perimeter for the Structural Wedge. }\end{array}$ \\
\hline 4 & $D$ & 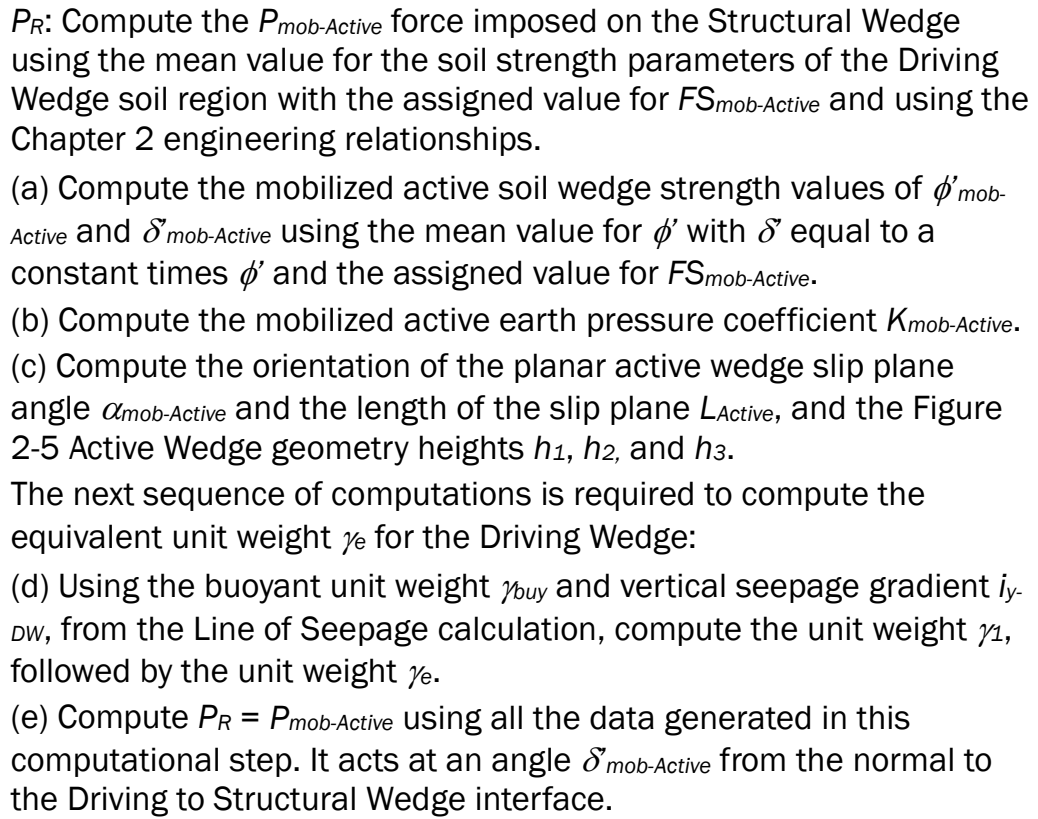 \\
\hline
\end{tabular}




\begin{tabular}{|c|c|c|}
\hline Step & $\begin{array}{l}\text { Driving, } \\
\text { Resisting or } \\
\text { Structural } \\
\text { Wedge(s) }\end{array}$ & Description \\
\hline 5 & $R$ & 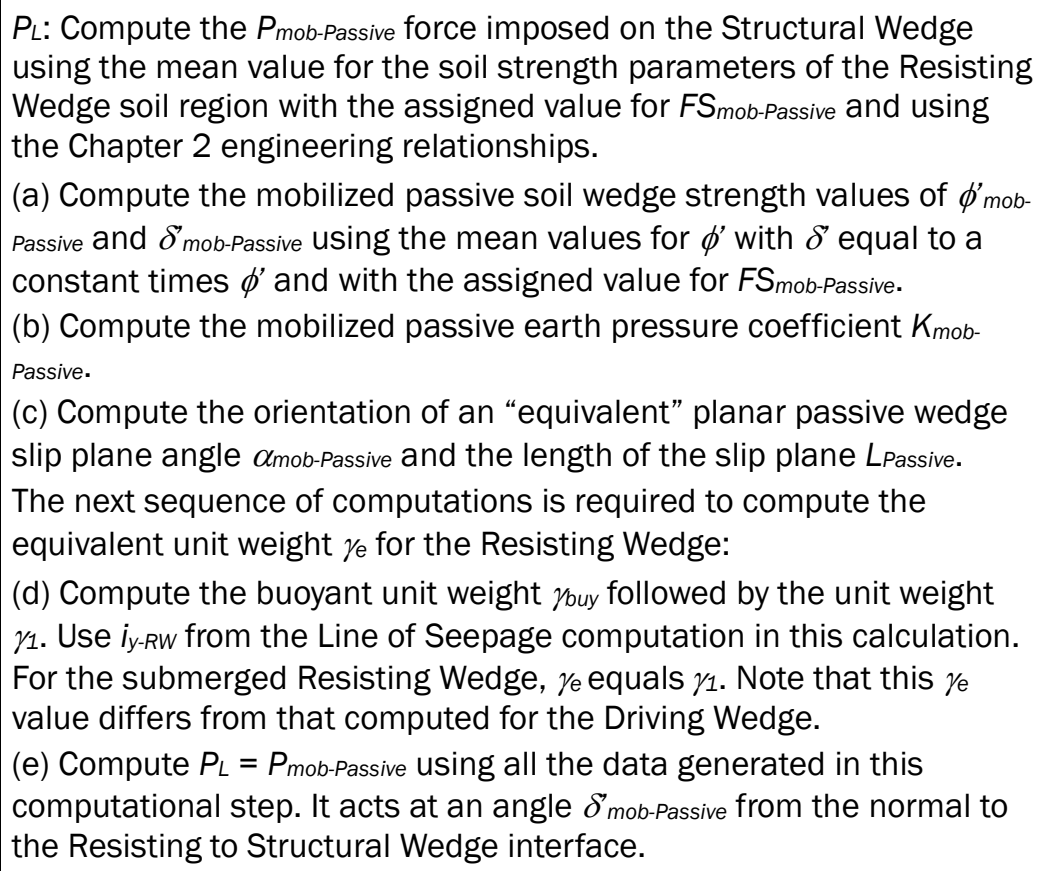 \\
\hline 6 & $S$ & $\begin{array}{l}\text { Boundary Water pressures, } u \text { : Using the results from the Line of } \\
\text { Seepage analysis: (a) compute the distribution of boundary water } \\
\text { pressures acting normal to the left side, base and right side faces of } \\
\text { the structural wedge. } \\
\text { (b) Using this distribution of } u \text {, compute the resultant boundary water } \\
\text { pressure forces acting normal to the three faces of the Structural } \\
\text { Wedge, } H_{L}, U_{\text {base }} \text { and } H_{R} \text {. }\end{array}$ \\
\hline 7 & $S$ & $\begin{array}{l}\text { Check for gap: } \\
\text { (a) Use the vertical force equilibrium equation given in Chapter } 2 \text { to } \\
\text { solve for the magnitude of the effective normal force } N^{\prime} \text { acting normal } \\
\text { to the base of the Structural Wedge. } \\
\text { (b) Solve for the location of } N^{\prime} \text { as measured from the Toe of the } \\
\text { Structural Wedge, } x_{N \text {-base. }} \\
\text { (c) Compute the length of gap } L_{g a p},(\text { if any). } \\
\text { (d) Compute the base within compression, } B_{e} \text {. Note that } B_{e} \text { is always } \\
\text { less than or equal to Base. }\end{array}$ \\
\hline 8 & $D, S, R$ & $\begin{array}{l}\text { If a gap is computed in the previous step, repeat the analysis starting } \\
\text { with the Line of Seepage analysis and include this gap length: If the } \\
\text { previous step results in a nonzero gap length, repeat steps ( } 3 \text { ) } \\
\text { through ( } 7) \text { until convergence or a minimum tolerance in } L \text { gap is met. } \\
\text { For the example problem, the tolerance for convergence on gap length } \\
\text { is given as } 0.1 \mathrm{ft} \text {. }\end{array}$ \\
\hline
\end{tabular}




\begin{tabular}{|l|l|l|}
\hline Step & $\begin{array}{l}\text { Driving, } \\
\text { Resisting or } \\
\text { Structural } \\
\text { Wedge(s) }\end{array}$ & Description \\
\hline 9 & S & $\begin{array}{l}\text { Compute } F S_{\text {foundation: }} \\
\text { (a) With all the forces acting on the Structural Wedge defined, solve } \\
\text { for the shear force } T_{\text {mob }} \text { required for equilibrium along the base of the } \\
\text { Structural Wedge using Equation 2.30. } \\
\text { (b) Compute the factor of safety against sliding along the base of the } \\
\text { structural wedge. Calculate FS Foundation using Equation 2.32 with the } \\
\text { ultimate shear force capacity } T_{\text {ult }} \text { given by Equation 2.31. }\end{array}$ \\
\hline 10 & D, S, R & $\begin{array}{l}\text { Iterate on factor of safety: If the computed value for FS foundation does } \\
\text { not match that assumed for FS } \text { mob-Active and FS } F \text { mob-Passive, repeat steps } \\
\text { (3) through (9) using an adjusted factor of safety value. This iterative } \\
\text { process continues until convergence within a minimum tolerance in } \\
\text { factor of safety is met. For the example problem, convergence is } \\
\text { determined when the newly generated Factor of Safety varies by a } \\
\text { tolerance value less than 0.01 Recall that for the Normal load case a } \\
\text { minimum value of 1.5 is required for factor of safety against sliding. }\end{array}$ \\
\hline
\end{tabular}

Step 10 is complicated because Equation 2.32 will go to infinity as $T_{m o b}$ approaches a zero value for the numerical modeling in the simulation process. This occurs because the mobilized Resisting Wedge force sufficiently counters the mobilized Driving Wedge force. It is further complicated because values for the soil strength properties can cause the Sliding Limit State to occur in the opposite direction than intended, toward the Driving Wedge. These conditions mean that it is important to choose a range to search for the maximum value of $F S_{\text {foundation }}$ equivalent to $F S_{\text {Active }}$ and $F S_{\text {Passive }}$ (within the tolerance of 0.01). This range can be obtained by finding two bounding values of $F S_{\text {Active }}$, with $F S_{\text {Passive }}$ being set equal to FS Active. The first bounding value is the $F S_{\text {Active }}$ value that generates (using Steps 3-9) a value of $F S_{\text {foundation, which is greater } F S_{\text {Active. }} \text { The second }}$ bounding value has to be chosen such that the computed value of $F S_{\text {foundation }}$ is less than $F S_{\text {Active }}$ and greater than zero. When the bounds have been chosen, the iteration step, Step 10, can be accomplished using a numerical search algorithm within the bounds.

\subsubsection{Engineering steps in the calculation of COVspatial for the Driving and Resisting Wedges and the Foundation Soil under the sliding limit state}

In the normal load case (EM 1110-2-2502) (HQUSACE 1989), the T-Wall design for the sliding limit state centers on the application of earth pressures by the Resisting Wedge and Driving Wedge (when present) to the Structural Wedge. The calculation steps of this $C O V_{\text {spatial }}$ for the three soil 
wedges is described in Table 3-5. The sliding limit state procedure of analysis of the Driving, Structural, and Resisting Wedges is summarized in Table 3-4. These calculations make use of mean values for $\phi$ ' and $\delta$ in each of the three soil regions in Table 3-5 to compute the length of the Driving Wedge slip plane $L_{\text {Active }}$ and the length of the Resisting Wedge slip plane $L_{\text {Passive. The objective of these computations is to define } C O V_{\text {spatial }} \text { values for }}$ each of the three soil regions for use in the Reliability analysis of the subsequent subsection.

Table 3-5. Procedure to calculate $C O V_{\text {spatial }}$ for the Driving and Resisting Wedges and for the foundation soil.

\begin{tabular}{|c|c|c|}
\hline Step & $\begin{array}{l}\text { Driving, } \\
\text { Resisting or } \\
\text { Structural } \\
\text { Wedge(s) }\end{array}$ & Description \\
\hline 1 & $D, S, R$ & $\begin{array}{l}\text { Determine soil wedge interactions: iterate through Table 3-4, } \\
\text { assigning mean values to } \phi^{\prime} \text { and } \delta^{\prime} \text { for each soil region. }\end{array}$ \\
\hline 2 & $D$ & $\begin{array}{l}\Gamma^{2}(L) \text { Driving Wedge: Compute the Variance Reduction Factor for the } \\
\text { retained soil. } \\
\text { (a) Using the vertical and horizontal scales of fluctuation }\left(\delta \text { and } \delta_{n}\right) \\
\text { for the retained soil (Table } 2-2) \text {, compute the equivalent scale of } \\
\text { fluctuation } \delta_{E} \text { along the planar slip plane of length } L=L_{\text {Active }} \text { at angle } \\
\alpha_{\text {mob-Active. }} \\
\text { (b) Compute the distance factor } n \text { using the Figure } 2-4 \text { relationship of } \\
n=L_{\text {Active }} / \delta_{E} \\
\text { (c) Determine the value for the Variance Reduction Factor using Figure } \\
2-4 \text {. }\end{array}$ \\
\hline 3 & $D$ & $\begin{array}{l}\mathrm{COV}_{\text {spatial }} \text { Driving Wedge: Compute } \mathrm{COV}_{\text {spatial }} \text { of the effective angle of } \\
\text { internal friction for the active wedge using the } \mathrm{COV}_{\text {point estimate value }} \\
\text { given in Table } 2-1 .\end{array}$ \\
\hline 4 & $R$ & $\begin{array}{l}\Gamma^{2}(L) \text { Resisting Wedge: Compute the Variance Reduction Factor for the } \\
\text { buttressing soil. } \\
\text { (a) Using the vertical and horizontal scales of fluctuation }\left(\delta \text { and } \delta_{n}\right) \\
\text { for the buttressing soil (Table } 2-2) \text {, compute the equivalent scale of } \\
\text { fluctuation } \delta_{E} \text { along the "approximate" planar slip plane of length } L= \\
L_{\text {Passive }} \text { at angle } \alpha_{\text {mob-Passive. }} \\
\text { (b) Compute the distance factor } n \text { using the Figure } 2-4 \text { relationship of } \\
n=L \text { Passive } \delta_{E} \\
\text { (c) Determine the value for the Variance Reduction Factor using Figure } \\
2-4 \text {. }\end{array}$ \\
\hline 5 & $R$ & 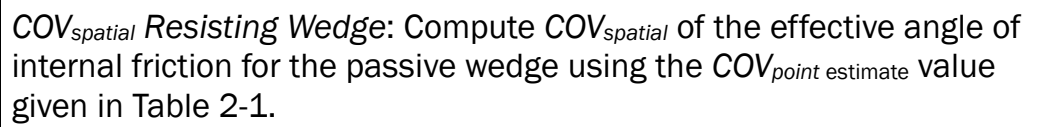 \\
\hline
\end{tabular}




\begin{tabular}{|l|l|l|}
\hline Step & $\begin{array}{l}\text { Driving, } \\
\text { Resisting or } \\
\text { Structural } \\
\text { Wedge(s) }\end{array}$ & Description \\
\hline 6 & $\mathrm{~S}$ & $\begin{array}{l}\Gamma^{2}(L) \text { Structural Wedge: Compute the Variance Reduction Factor for } \\
\text { the foundation soil. } \\
\text { (a) Using the horizontal scale of fluctuation }\left(\delta_{h}\right) \text { for the foundation soil } \\
\text { (Table 2-2) and base width } L=\text { Base, compute the distance factor } n \\
\text { using the Figure 2-4 relationship of } n=\text { Base / } \delta \\
\text { (b) Determine the value for the Variance Reduction Factor using } \\
\text { Figure 2-4. }\end{array}$ \\
\hline 7 & $\mathrm{~S}$ & $\begin{array}{l}\text { COV } \text { spatial Structural Wedge: Compute } C O V_{\text {spatial of the effective angle of }} \\
\text { internal friction for the foundation using the COV } V_{\text {point estimate value given }} \\
\text { in Table 2-1. }\end{array}$ \\
\hline
\end{tabular}

\subsubsection{Engineering steps in the Reliability analysis of the Structural Wedge for the sliding limit state}

In a Reliability analysis of the sliding limit state of the Figure 2-3 Structural Wedge, the variability in the soil shear strength parameters is considered. Table 2-1 lists the mean and point estimate COV values for the effective angle of internal friction and interface friction for the three soil regions of the Figure 2-1 T-Wall example problem model. Performing the series of computations outlined in Subsection 3.3.1 results in the transformation of these Table 2-1 point estimate COV values into spatial COV values for each soil region, as required for use in Geotechnical limit state analyses (Ebeling and White 2019). The engineering computations, used in the probabilistic sliding limit state procedure of Reliability analysis of the Structural Wedge, are summarized in Table 3-6. 
Table 3-6. Step-by-step sliding limit state procedure of Reliability analysis of the Structural Wedge.

\begin{tabular}{|c|c|c|}
\hline Step & $\begin{array}{l}\text { Driving, } \\
\text { Resisting or } \\
\text { Structural } \\
\text { Wedge(s) }\end{array}$ & Description \\
\hline 1 & $D, S, R$ & $\begin{array}{l}\text { Determine soil wedge interactions: iterate through Table } 3-4 \text {, } \\
\text { assigning mean values to } \phi^{\prime} \text { and } \delta^{\prime} \text { for each soil region. }\end{array}$ \\
\hline 2 & $D$ & $\begin{array}{l}\text { Driving force } L \text { : Compute the Driving force } L \text { for simulation } i \text { using } \\
\text { Equation 3.7. }\end{array}$ \\
\hline 3 & $R$ & $\begin{array}{l}P_{L} \text { : Compute the ultimate } P_{\text {Passive force imposed on the Structural }} \\
\text { Wedge using the simulated value for the soil strength parameters of } \\
\text { the Resisting Wedge soil region using the Chapter } 2 \text { engineering } \\
\text { relationships. } \\
\text { (a) Define the ultimate passive soil wedge strength values of } \phi^{\prime} \text { 'Passive } \\
\text { and } \delta^{\prime} \text { Passive using the simulation generated value for } \phi^{\prime} \text {, with } \delta^{\prime} \text { equal } \\
\text { to a constant times } \phi^{\prime} \text {. } \\
\text { (b) Compute the ultimate passive earth pressure coefficient } K_{\text {Passive. }} \\
\text { (c) Compute the orientation of an "equivalent" planar passive wedge } \\
\text { slip plane angle } \alpha \text { Passive and the length of the slip plane } L_{\text {passive. }} \\
\text { The next sequence of computations is required to compute the } \\
\text { equivalent unit weight } \gamma_{e} \text { for the Resisting Wedge: } \\
\text { (d) Compute the buoyant unit weight } \gamma_{\text {buy }} \text { followed by the unit weight } \\
\gamma_{1} \text {. Use } i_{y-R w} \text { from the Line of Seepage computation in this calculation. } \\
\text { For the submerged Resisting Wedge, } \gamma_{e} \text { equals } \gamma_{1} \text {. Note that this } \gamma_{e} \\
\text { value differs from that computed for the Driving Wedge. } \\
\text { (e) Compute } P_{L}=P_{\text {Passive }} \text { using all the data generated in this } \\
\text { computational step. It acts at an angle } \delta^{\prime} \text { Passive from the normal to the } \\
\text { Resisting to Structural Wedge interface. }\end{array}$ \\
\hline 4 & $S$ & $\begin{array}{l}\text { Tult: Compute the ultimate horizontal shear force along the base of the } \\
\text { structural Wedge using Equation } 2.31 \text { with ultimate shear strength } \\
\text { soil parameters for the foundation soil. The computation of the } \\
\text { effective normal force } N \text { ' is given by Equation } 2.29 \text {. }\end{array}$ \\
\hline 5 & $S, R$ & $\begin{array}{l}\text { Resisting force } R \text { : Compute the Resisting force } R \text { for simulation } i \text { using } \\
\text { Equation 3.8. }\end{array}$ \\
\hline 6 & $D, S, R$ & $\begin{array}{l}\text { Assessing } g(X) \text { : Compute the value of the performance function } g(X) \\
\text { for this simulation } i \text { using Equation } 2.52 \text {. Note that if } g(X) \text { is positive, } \\
\text { then the performance of the structure is counted as satisfactory } \\
\text { against the base area in compression limit state for variable vector } X \text {. } \\
\text { However, if } g(X) \text { is negative, then the performance of the structure is } \\
\text { counted as unsatisfactory against the base area in compression limit } \\
\text { state for variable vector } X \text {. }\end{array}$ \\
\hline
\end{tabular}


Step 1 follows the procedure of Table $3-4$, assigning the simulation values of $\boldsymbol{X}$ for the variable soil properties of the wedges. The next step is to collect the Figure 2-10 Driving forces into a single force term and then do the same for the horizontal Resisting forces of Equation 2.30. The following relationship is used in Step 2 to compute the horizontal resultant driving force $L$ imposed on the Structural Wedge by the Driving Wedge after the iterative computational procedure of Table 3-4 has been completed. The Equation 2.30 driving forces summed and designated as $L$, are

$$
L=H_{R}+E_{R}
$$

In the probabilistic simulation process, the force $H_{R}$ is deterministic. The other horizontal effective earth pressure force $E_{R}$ is variable and will change in value during each simulation. Its value is determined from the $P_{R}$ value. $P_{R}$ is set equal to $P_{A}$ from Equation 2.4 and is computed using the mobilized effective angle of internal friction of the soil, $\phi^{\prime}$ mob-Active, and the mobilized interface angle of friction, $\delta^{\prime}$ mob-Active for the last FS iteration. Note that the mobilized values for $\phi^{\prime}$ and $\delta$ are being used in conjunction with this $P_{R}$ value to compute the $E_{R}$ and $X_{R}$ forces the Driving Wedge imposes on the Structural Wedge and not their ultimate strength values. The horizontal force component of mobilized force $P_{R}$ is given as

$$
E_{R}=P_{R} * \cos \left(\delta^{\prime}{ }_{m o b-A c t i v e}\right)
$$

The vertical component of the mobilized right-hand side driving (wedge) force is given by

$$
X_{R}=P_{R} * \sin \left(\delta_{\text {mob-Active }}^{\prime}\right)
$$

The horizontal resisting forces, designated as $R$, are given as

$$
R=H_{L}+E_{L}+T_{u l t}
$$

In the probabilistic simulation process used in Table $3-6$, the force $H_{L}$ is deterministic. The other two forces $E_{L}$ and $T_{u l t}$ are variable and will change in value during each simulation. The horizontal component of the ultimate left-hand side ultimate resisting force $P_{L}$ provided by the Resisting Wedge to the Structural Wedge given by

$$
E_{L}=P_{L} * \cos \left(\delta^{\prime}\right)
$$


In this equation, $P_{L}$ is set equal to $P_{P}$ from Equation 2.15 and is computed using the effective angle of internal friction of the soil, $\phi^{\prime}$ Passive, and the interface angle of friction, $\delta^{\prime}$ Passive (Step 3). Observe that the ultimate values for $\phi$ ' and $\delta$ are being used and not their mobilized values to compute $E_{L}$.

The vertical component of the ultimate left-hand side resisting force $P_{L}$ provided by the Resisting Wedge is given by

$$
X_{L}=P_{L} * \sin \left(\delta^{\prime}\right)
$$

Recall from Chapter 2 that the summation of vertical forces acting on the Figure 2-10 T-Wall results in the expression for the effective vertical force normal to the base of the T-Wall,

$$
N^{\prime}=W-U_{\text {base }}+X_{R}-X_{L}
$$

The ultimate horizontal force (i.e., capacity) along the base of the structural wedge is computed in Step 4 using the relationship

$$
T_{u l t}=c^{\prime} * B_{e}+N^{\prime} \tan \left(\phi^{\prime}\right)
$$

where $c$ ' is the effective cohesion, $B_{e}$ is the base length of the Structural Wedge in compression, and $\phi$ ' is the effective angle of internal friction. The Step 4 computation of the effective normal force $N^{\prime}$ is given by Equation 2.29. With $E_{L}$ and $T_{u l t}$ now defined, $R$ may be computed using Equation 3.7 in Step 5 of Table 3-6.

The value of the performance function $g(\boldsymbol{X})$ for simulation $i$ is computed in Step 5 using Equation 2.52. Table 3-6 is repeated for simulations $i+1$ through $n$ simulations. In Figure 3-1, all of the computed forces that are acting against the two-dimensional (2-D) cross section of the wall and its components are shown. 
Figure 3-1. Free body diagram of resultant forces acting on the stem and base slab of the Structural Wedge.

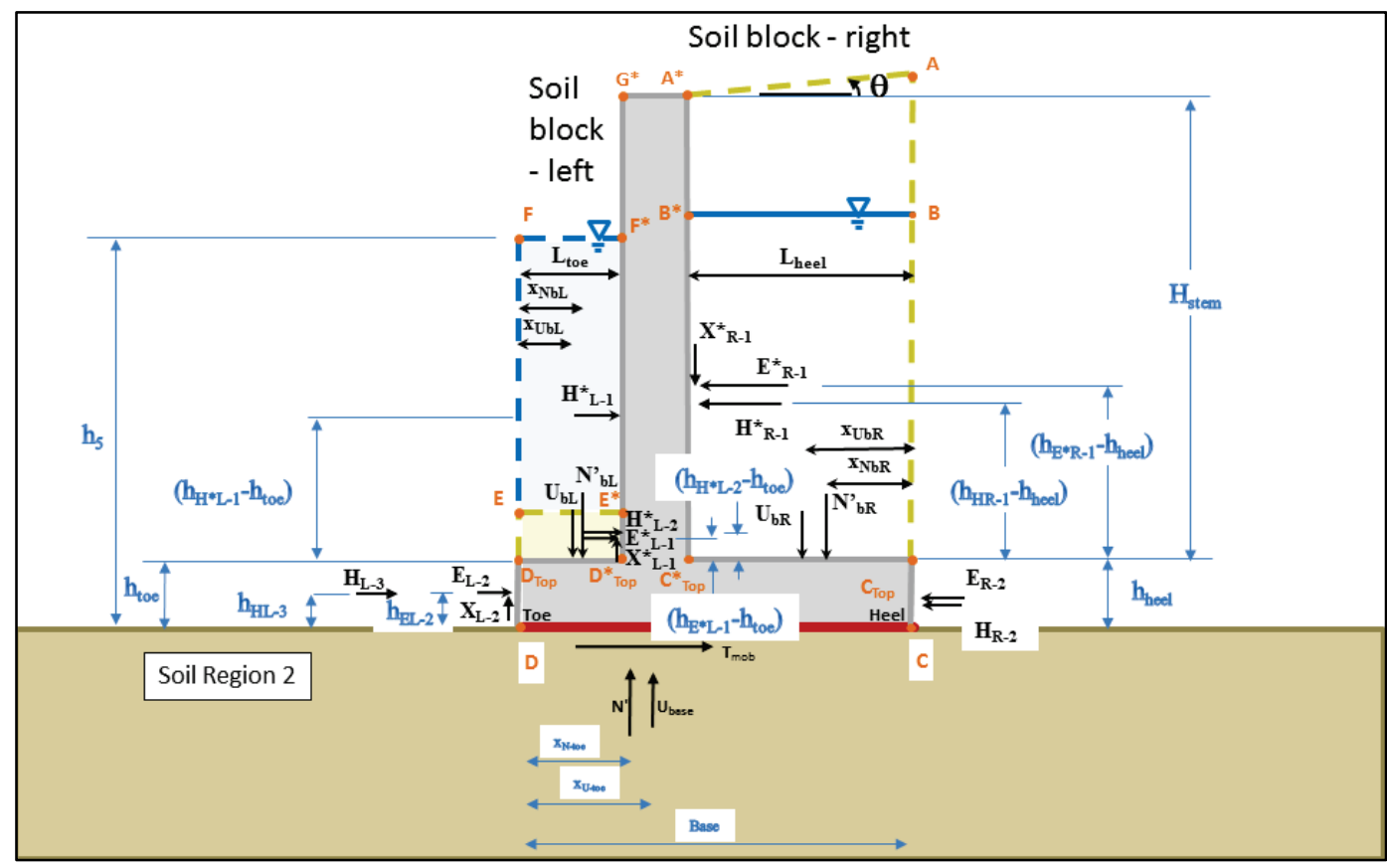

\subsection{Bearing failure of the Structural Wedge limit state}

In Subsection 3.1, the third limit state deals with bearing failure of the structural system beneath its foundation. In the USACE design process for hydraulic structures, this limit state is assessed based on several factors for the Driving, Structural, and Resisting Wedges. This subsection summarizes the required steps: (1) a deterministic analysis to convert $C O V_{\text {point estimates }}$ to $C O V_{\text {spatial }}$ for the effective angle of internal soil friction of the retained soil of the Structural Wedge and for the buttressing soil around the Structural Wedge and (2) a procedural method to compute the performance of a structural system for the bearing limit state.

\subsubsection{Iterative method for determining interactions between Driving, Resisting, and Structural Wedges for force equilibrium conditions for bearing limit state}

For the bearing limit state, the force configuration acting on the Structural Wedge has the equivalence constraints for FS as the sliding limit state. Therefore, the same procedure for interaction of the wedges (Table 3-4) applies for this limit state and the calculation of the effective base width $B_{e}$ and the vertical shear forces, which act at the interfaces of the Structural Wedge. 


\subsubsection{Engineering steps in the calculation of $\operatorname{COV}_{\text {spatial }}$ for the foundation soil}

The normal load case (EM 1110-2-2502) (HQUSACE 1989) of the T-Wall design for the bearing limit state centers on the application of the vertical pressure created by the weight of and shear forces applied to the Structural Wedges along the base of the structure. Because shear forces and the effective base length are required to determine the pressures acting at the base, a full analysis using the iterative method must be performed as shown in Table 3-5. This means that the spatial variance in the Driving and the Resisting Wedge must be determined as before. The lengths of the slip planes of the Resisting and Driving Wedges are necessary to determine the geospatial coefficient of variation for Reliability analysis from the point estimate coefficient of variation and are calculated according to the same procedure as before.

For bearing, the variance of the soil beneath the structure is determined in a different fashion, instead of using a slip plane defining a linear wedge. In this circumstance, the soil is being pushed downward and, because of resistance below, outward to the sides of the structure. Figure 3-2 shows the zones of shear for the strip footing Structural Wedge being punched into the soil (Vesic 1967).

Vanmarcke (1977a) suggested a fitting of the space with a theoretical triangular function on one side or the other of the structure. For example, the triangular region would be Regions I, II, and III on either the left or right of the structure in Figure 3-2, which is not to scale. He also suggested that an approximate simplified variance reduction factor for the base soil could be calculated as the product of the variance reduction factors for the vertical and horizontal scale of fluctuation directions. This approximation is followed by Baker and Calle (2002), Schneider and Schneider (2013), Babu and Dasaka (2007).

$$
\Gamma_{A}^{2}=\Gamma_{v}^{2} \times \Gamma_{h}^{2}
$$

Babu and Dasaka (2007) suggested that, for cohesionless soils, the depth for vertical load regions could be approximated by $2 B$, where $B$ is the length of the footing base, and the horizontal region distance for spatial approximation would be $2.5 B$ along the horizontal axis. Extending the approximation for soil that can move to both sides of the structure implies that the horizontal soil region spatial distance should be $2 \times 2.5 B$, or $5^{B}$. 
Babu (2014) changed this horizontal spatial dimension for shallow foundations to $3.5 B$ for a single side or $7 B$ for the entire horizontal component, and these are the values the authors adopted for the shallow embedment of the T-Wall example problem.

Figure 3-2. Idealizations of shear zones at failure of an earth-supported strip footing Structural Wedge. Zone I: Rankine Active zone; Zone II: Rankine Passive zone; and Zone III: Radial Shear zone.

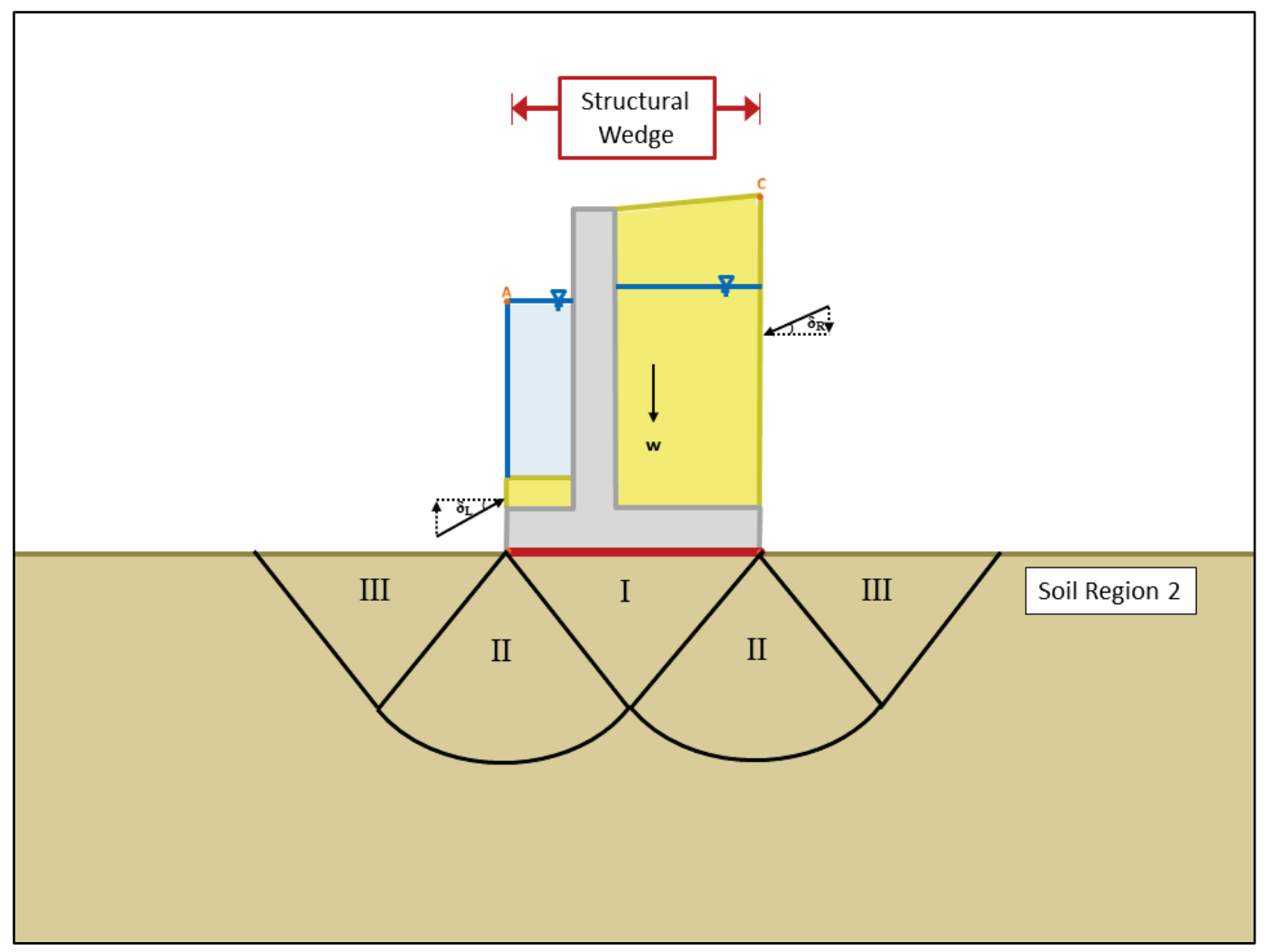

The step-by-step calculation of $C O V_{\text {spatial }}$ for the bearing limit state procedure of analysis of the Structural Wedge is summarized in Table 3-7, as well as the method to determine the slip planes for the wedges and the soil beneath the base of the structure. The bearing limit state procedure of analysis of the Driving, Structural, and Resisting Wedges is summarized in Table 3-4.These calculations make use of mean values for $\phi$ ' and $\delta$ in each of the three soil regions to compute the length of the Driving Wedge slip plane $L_{\text {Active }}$, the length of the Resisting Wedge slip plane $L_{\text {Passive }}$ and $L_{V}$ and $L_{H}$ for the foundation soil below the Structural Wedge. 
Table 3-7. Step-by-step base area in compression limit state procedure of analysis of the Structural Wedge to calculate $C_{\text {SOV }}$ spatial for the Driving and Resisting Wedges.

\begin{tabular}{|c|c|c|}
\hline Step & $\begin{array}{l}\text { Driving, } \\
\text { Resisting or } \\
\text { Structural } \\
\text { Wedge(s) }\end{array}$ & Description \\
\hline 1 & $\mathrm{D}, \mathrm{S}, \mathrm{R}$ & $\begin{array}{l}\text { Determine soil wedge interactions: iterate through Table 3-4, } \\
\text { assigning mean values to } \phi^{\prime} \text { and } \delta^{\prime} \text { for each soil region. }\end{array}$ \\
\hline 2 & $\mathrm{D}$ & $\begin{array}{l}\Gamma^{2}(L) \text { Driving Wedge: Compute the Variance Reduction Factor for the } \\
\text { retained soil. } \\
\left.\text { (a) Using the vertical and horizontal scales of fluctuation ( } \delta \text { and } \delta_{h}\right) \\
\text { for the retained soil (Table } 2-2) \text {, compute the equivalent scale of } \\
\text { fluctuation } \delta_{\text {E }} \text { along the planar slip plane of length } L=L_{\text {Active }} \text { at angle } \\
\alpha_{\text {mob-Active. }} \\
\text { (b) Using Equation } 2.1 \text { with } L=L_{\text {Active, }} \text { compute the Variance Reduction } \\
\text { Factor for the retained soil. }\end{array}$ \\
\hline 3 & $D$ & $\begin{array}{l}C O V_{\text {spatial }} \text { Driving Wedge: Compute } \mathrm{COV}_{\text {spatial }} \text { using Equation } 2.2 \text { of the } \\
\text { effective angle of internal friction for the active wedge using the } \\
C O V_{\text {point estimate value given in Table } 2-1 .}\end{array}$ \\
\hline 4 & $\mathrm{R}$ & 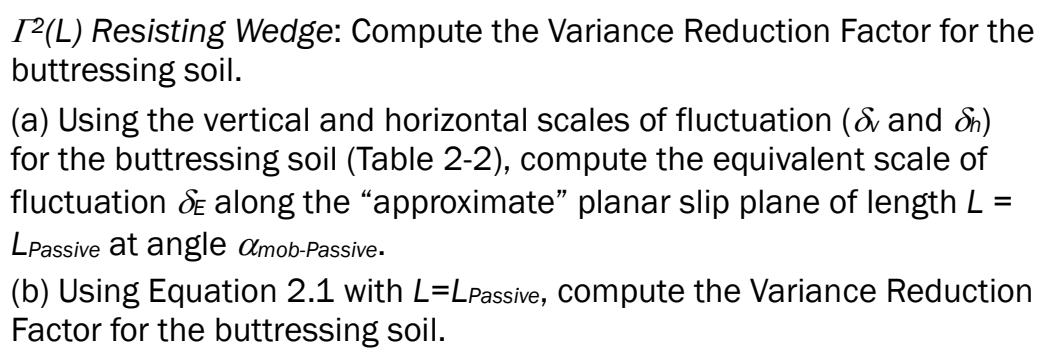 \\
\hline 5 & $\mathrm{R}$ & $\begin{array}{l}C O V_{\text {spatial }} \text { Resisting Wedge: Compute } \text { COV }_{\text {spatial }} \text { using Equation } 2.2 \text { of } \\
\text { the effective angle of internal friction for the passive wedge using the } \\
\text { COV }_{\text {point estimate value given in Table } 2-1 .}\end{array}$ \\
\hline 6 & $\mathrm{~S}$ & 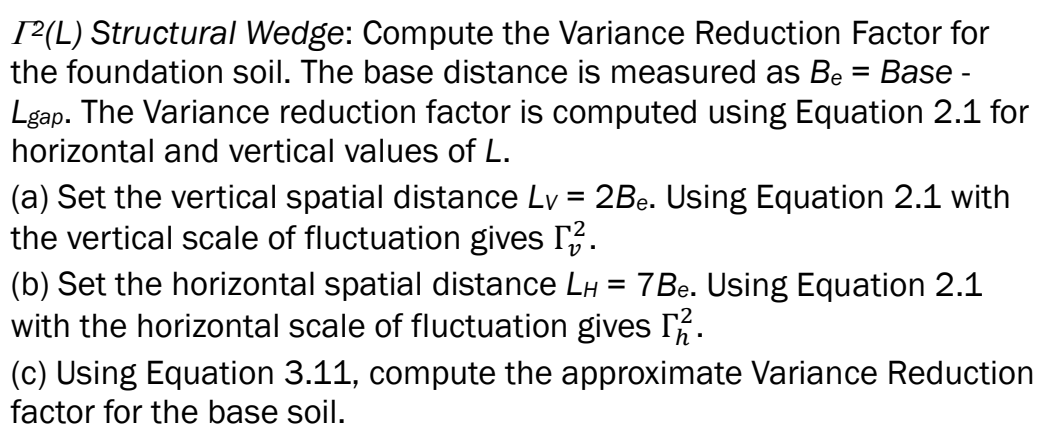 \\
\hline 7 & $\mathrm{~S}$ & $\begin{array}{l}\mathrm{COV}_{\text {spatial }} \text { Structural Wedge: Compute } \mathrm{COV}_{\text {spatial }} \text { using Equation } 2.2 \text { of } \\
\text { the effective angle of internal friction for the foundation soil using the } \\
C O V_{\text {point estimate value given in Table } 2-1 .}\end{array}$ \\
\hline
\end{tabular}




\subsubsection{Engineering steps to assess the performance (satisfactory/unsatisfactory) of the Structural Wedge for the bearing limit state}

For a Reliability analysis of the bearing limit state of the Figure 2-3 structural wedge, variability in the soil shear strength parameters are considered. Table 2-1 lists the mean and point estimate COV values for the effective angle of internal friction and interface friction for the three soil regions of the Figure 2-1 T-Wall example problem model. Performing the series of computations outlined in Subsection 3.4.1 results in the transformation of these Table 2-1 point estimate COV values into spatial $\mathrm{COV}$ values for each soil region, as required for use in Geotechnical limit state analyses (Ebeling and White 2019). Table 3-8 establishes the steps to compute the performance of a designed T-Wall given a vector of random variables $(\boldsymbol{X})$ determined using the $C O V_{\text {spatial }}$ values from Table 3-7. For reliability purposes, this function is called the $g(\boldsymbol{X})$ function, as will be discussed in Chapter 4. For the sample T-Wall example, this vector is comprised of the $\phi$ and $\delta$ values for the soil properties in the Driving, Resisting, and beneath the Structural Wedges. For a reliability analysis, a set of vectors will be created, and this function will be used with each of these vectors to determine PUP.

Table 3-8. Step-by step-base area in compression limit state procedure of Reliability analysis of the Structural Wedge.

\begin{tabular}{|l|l|l|}
\hline Step & $\begin{array}{l}\text { Driving, } \\
\text { Resisting or } \\
\text { Structural } \\
\text { Wedge(s) }\end{array}$ & Description \\
\hline 1 & D, S, R & $\begin{array}{l}\text { Determine soil wedge interactions: iterate through Table 3-4, } \\
\text { assigning mean values to } \phi^{\prime} \text { and } \delta \text { for each soil region. }\end{array}$ \\
\hline 2 & D, S, R & $\begin{array}{l}\text { Resisting force } R \text { : Compute the resisting force using Equations D.1 } \\
\text { and D.2, which implement uniform base pressure capacity. }\end{array}$ \\
\hline 3 & D, S, R & $\begin{array}{l}\text { Load force } L: \text { Compute the vertical load } L \text { for simulation } i \text { using } \\
\text { Equation 3.12. }\end{array}$ \\
\hline 4 & D, S, R & $\begin{array}{l}\text { Assessing } g(X): \text { Compute the value of the performance function } g(X) \\
\text { for this simulation } i \text { using Equation } 2.52 . \text { Note that if g }(X) \text { is positive } \\
\text { then the performance of the structure is counted as satisfactory } \\
\text { against the base area in compression limit state for variable vector } X \text {. } \\
\text { However, if } g(X) \text { is negative then the performance of the structure is } \\
\text { counted as unsatisfactory against the base area in compression limit } \\
\text { state for variable vector } X \text {. }\end{array}$ \\
\hline
\end{tabular}


The equations for calculating the resistance base pressure and the subsequent resisting force, assuming that the pressure is applied uniformly, are Equation D.1 and Equation D.2 of Appendix D in this report. The pressures are assumed to be applied uniformly across the base because the failure constraint is that foundation soil has developed a fully plastic state with the full mobilization of the shear strength. This allows the entire structure to punch into the foundation soil as idealized in Figure 3-2.

$$
q=\zeta_{c} \zeta_{c d} \zeta_{c i} \zeta_{c t} \zeta_{c g} c N_{c}+\zeta_{q} \zeta_{q d} \zeta_{q i} \zeta_{q t} \zeta_{q g} q_{0} N_{q}+\frac{\zeta_{\gamma} \zeta_{\gamma d} \zeta_{\gamma i} \zeta_{\gamma t} \zeta_{\gamma g} B \gamma N_{\gamma}}{2} \text { (D.1 bis) }
$$

where

$q$ = vertical component of the ultimate unit bearing capacity of the foundation (pressure)

$N_{c}, N_{q}, N_{\gamma}=$ base bearing capacity factors

$\zeta_{c}, \zeta_{q}, \zeta_{\gamma}=$ shape factors

$\zeta_{c d}, \zeta_{q d}, \zeta_{\gamma d}=$ embedment factors

$\zeta_{c i}, \zeta_{q i}, \zeta_{Y I}=$ inclination factors

$\zeta_{c t}, \zeta_{q t}, \zeta_{\gamma t}=$ base tilt factors

$\zeta_{c g}, \zeta_{q g}, \zeta_{\gamma g}=$ ground slope factors

$C=$ cohesion

$\gamma=$ unit weight of the soil

$B_{e}=$ effective base width

$L=$ effective base length (for a $2 \mathrm{D}$ section evaluation, a value of 1.0 is used)

$q o=$ effective overburden pressure on a plane passing through the base of the footing.

Notice that each of the factor terms is defined based on the variable properties of the foundation soil, the effective base width, and the geometry of the structure. These definitions are in Appendix D. The effective base width is altered by the variable soil properties in the Driving and Resisting Wedges.

Because the computations used for Sliding and Base Area in Compression (Overturning) limit states already provide the effective vertical force exhibited by the structural wedge, which acts directly against the base, the bearing capacity is converted to an ultimate resisting force for the effective base width (for the 2-D slice), which is the width of the base in contact with the foundation soil. This width will be affected by uplift and overturning 
forces that cause a gap to form between the foundation soil and the foundation.

$$
R_{\text {Bearing }}=B_{e} e q
$$

The Equation 2.36 vertical driving load, designated as $L$, is

$$
L=W-U_{\text {base }}+X_{R}-X_{L}
$$

Observe that the vertical demand $L$ is the only force that can cause a bearing failure below the structural wedge base. Because of the assumptions of uniform pressures, the position of this force does not matter. The $g(\boldsymbol{X})$ condition is only concerned that the total vertical forces on the structure exceed the resultant resistance bearing force computed in Equation D.2. The forces vary as the soil material properties $\phi$ ' and $\delta$ vary for both the load (in the $X r$ and $X l$ terms) and the bearing resistance.

Step 1 follows the procedure of Table 3-4, assigning the simulation values of $\boldsymbol{X}$ for the variable soil properties of the wedges. Note that the mobilized values for $\phi^{\prime}$ and $\delta$ are being used in conjunction with this $P_{R}$ value to compute the $X_{R}$ forces the Driving Wedge imposes on the Structural Wedge and not their ultimate strength values. The vertical component of the mobilized right-hand side driving (wedge) force is given by

$$
X_{R}=P_{R} * \sin \left(\delta^{\prime}{ }_{m o b-A c t i v e}\right)
$$

Similarly, the mobilized values for $\phi^{\prime}$ and $\delta$ are being used in conjunction with this $P_{L}$ value to compute the $X_{L}$ forces the Resisting Wedge imposes on the Structural Wedge and not their ultimate strength values. The vertical component of the mobilized left-hand side resisting (wedge) force is given by

$$
X_{L}=P_{L} * \sin \left(\delta_{\text {mob-Passive }}^{\prime}\right)
$$

The value of the performance function $g(\boldsymbol{X})$ for simulation $i$ is computed in Step 4 using Equation 2.52. The procedure of Table 3-8 is repeated for simulations $i+1$ through $n$ simulations to determine reliability of the structure under the bearing limit state. 
The step-by-step engineering computations, used in the base area in the probabilistic compression limit state procedure of Reliability analysis of the Structural Wedge, is summarized in Table 3-8.

\subsection{Shear failure of the wall stem limit state}

The previous limit states have been concerned with only the geotechnical portion of the Structural System. To that end, all of the forces have only been affecting the full Structural Wedge, which incorporates the actual structural feature of the T-Wall and any soil or water regions that lie directly above the base of the reinforced structural slab. The structural feature consists of a reinforced concrete base slab and a vertical reinforced concrete wall (referred to as the stem), which geometrically forms the T-shape of the T-Wall structure, as illustrated in Figure 2-3. The base slab bears the weight of the overburden, which helps prevent overturning and sliding, as discussed in Subsections 3.3 and 3.4, respectively. The vertical stem portion of the T-Wall bears the brunt of the horizontal earth and water pressures on the structural system. The bottom of the stem where it meets the base slab has the greatest load because the earth and water pressures increase with depth.

Determining the horizontal forces acting at the bottom of the stem wall requires a few steps: First, a geotechnical Wedge equilibrium analysis must be run converging to a common $F S$ value for the three wedges. This allows for the determination of the forces acting horizontally against the Structural Wedge imposed by the adjacent Driving and Resisting Wedges. This step follows the same procedure as Tables 3-4 and 3-6. These forces are combined with the model of the full Structural Wedge (with soils and water) to pass the forces to the base of the wall stem using the equations of Appendix B. Equation B.75 computes the geotechnical shear force at the wall stem, which is the load on the stem, based on the $\phi$ 'variables and the directly correlated $\delta$ values.

The structure's shear strength resistance is determined using the properties of the concrete used in the reinforced concrete structure. Reinforced concrete design of walls have been studied and typically have a variable strength using a lognormal distribution about the biased design strength of the structure, as given in Table 3-9. A bias factor is applied to the nominal design strength to account for conservatism in design. A bias factor of 1.15 is applied for shear strength calculations. Studies also reveal that the 
coefficient of variation for these structures is properly estimated to be 0.18 for concrete shear strength calculations.

Table 3-9. Design resistance model for the concrete Shear limit state (after Ellingwood ${ }^{1}$ ).

\begin{tabular}{|l|l|l|l|l|}
\hline Limit State & Distribution & $\begin{array}{l}\text { Mean bias } \\
\text { factor }\end{array}$ & $\begin{array}{l}\text { Coefficient of } \\
\text { Variation }\end{array}$ & $\begin{array}{l}\text { Standard } \\
\text { Deviation }\end{array}$ \\
\hline $\begin{array}{l}\text { Shear Capacity } \\
\text { (beams) }\end{array}$ & LogNormal & 1.15 & 0.18 & 0.207 \\
\hline
\end{tabular}

According to EM 1110-2-2104 (HQUSACE 2016), the load factor that should be applied to determine the design capacity of the structure under the usual case lateral earth loading is 2.2 (Table 3-1 of EM 1110-2-2104) (HQUSACE 2016). The required (minimum) reinforced concrete shear force value is computed for the lateral earth shear force imposed under the usual load case with mean values for the three $\phi$ ' and the correlated $\delta$ values for the soil wedges. The Table 3-9 load factor times the bias factor is applied to the mean concrete shear resistance capacity to arrive at the shear strength value used in the Ultimate limit state design.

The design resistance capacity and distribution (Table 3-9) are used to form a limit state $g(\boldsymbol{X})$ function with an analysis of the Structure under the Extreme load case using Tables 3-4 and 3-6 and Equation B.75. This equation has 4 variables: the previously existing variables $\phi^{\prime} D W, \phi^{\prime} S W$, and $\phi^{\prime} R W$, and now the new resistance variable $s R$. The soil friction angle computations combined with Equation B. 75 give the load acting on the wall stem, and $s R$ gives the true resistance. This new $g(\boldsymbol{X})$ function (where $g(X)$ $=L-s R$ ) can be used with the ASM, Monte Carlo, and simulation methods to determine the fitness of the structure using a PUP calculation.

\subsection{Flexural failure of the wall stem limit state}

In the same spirit as the shear failure of the wall stem, flexural failure also occurs near the base of the wall stem. This is especially true for the flexural limit state because the greatest moment arm occurs about the bottom of the

\footnotetext{
1 Ellingwood, B. R. 2015. Procedures for Developing Reliability-Based Load Criteria for Hydraulic Steel and Concrete Structures. Final letter report submitted to the URS Group, Inc. U.S. Army Corps of Engineers.

NOTE: From this point forward, the document will be referred to or cited as "Ellingwood."
} 
stem. A very similar procedure is used to calculate the resistance capacity against flexural failure.

Determining the flexural forces acting at the bottom of the stem wall requires a few steps: First, a geotechnical wedge equilibrium analysis must be run converging to a common $F S$ value for the three wedges. This allows for the determination of the forces acting horizontally against the Structural Wedge imposed by the adjacent Driving and Resisting Wedges and the height that the forces are applied, for moment arm computation. This step follows the same procedure as Tables 3-4 and 3-6. These forces and moment arms are combined with the model of the full Structural Wedge (with soils and water) to pass the forces and moments to the base of the wall stem using the equations of Appendix B. Equation B. 76 computes the (geotechnical based) moment applied at the wall stem, which is the load on the stem, based on the $\phi$ 'variables and the directly correlated $\delta$ 'values.

The structure's flexural resistance is determined using the properties of the concrete and reinforcement steel used in the reinforced concrete structure. Reinforced concrete design of walls have been studied and typically have a variable strength using a lognormal distribution about the biased design strength of the structure, as given in Table 3-10. A bias factor is applied to the nominal design strength to account for conservatism in design. A bias factor of 1.12 is applied for moment strength calculations. Studies also reveal that the coefficient of variation for these structures is properly estimated to be 0.14 for moment strength calculations.

Table 3-10. Design resistance model for the flexural limit state (after Ellingwood).

\begin{tabular}{|l|l|l|l|l|}
\hline Limit State & Distribution & $\begin{array}{l}\text { Mean Bias } \\
\text { Factor }\end{array}$ & $\begin{array}{l}\text { Coefficient of } \\
\text { Variation }\end{array}$ & $\begin{array}{l}\text { Standard } \\
\text { Deviation }\end{array}$ \\
\hline $\begin{array}{l}\text { Flexural } \\
\text { Capacity (one- } \\
\text { way slabs) }\end{array}$ & LogNormal & 1.12 & 0.14 & 0.157 \\
\hline
\end{tabular}


According to EM 1110-2-2104 (HQUSACE 2016), the load factor that should be applied to determine the design capacity of the structure under the Usual case lateral earth loading (which is used to compute moments) is 2.2 (Table 3-1 of the EM 1110-2-2104) (HQUSACE 2016). This required (minimum) reinforced flexural capacity value is computed for the lateral earth force and force application height imposed under the Usual load case with mean values for the three $\phi^{\prime}$ and the correlated $\delta$ values for the soil wedges. The Table 3-10 load factor times the bias factor is applied to the mean flexural resistance capacity to arrive at the flexural capacity value used in the Ultimate limit states design.

The design flexural resistance capacity and distribution (Table 3-10) are used to form a limit state $g(\boldsymbol{X})$ function with an analysis of the structure under the Extreme load case using Tables 3-4 and 3-6 and Equation B.76. This equation has four variables; the previously existing variables $\phi^{\prime} D W, \phi^{\prime} S w$, and $\phi^{\prime} R W$, and now the new resistance variable $f R$. The soil friction angle computations combined with Equation B.76 give the load acting on the wall stem, and $f R$ gives the true resistance. This new $g(\boldsymbol{X})$ function (where $g(X)=$ $L-f R)$ can be used with the ASM, Monte Carlo, and simulation methods to determine the fitness of the structure using a PUP calculation. 


\section{A Procedural Method for Computing Individual Limit State Probability of Unsatisfactory Performance (PUP) and Load Factors for the Example Soil- Structure Interaction of the T-Wall Model with Material Variability}

\subsection{Introduction and research}

Numerical Reliability-based methodologies beyond those discussed in Chapter 2 were also investigated as part of this research effort. Several numerical methods that may be used for calculating Load and Resistance scale factors for a single limit state are described in Appendix C. It is important to recognize the principal limitations for these numerical procedures:

1. They all require a mathematically defined, continuous PDF distributions for load and for resistance.

2. These procedures also require that load and resistance be uncorrelated. Independent load and resistance variables satisfy this requirement.

The first numerical method discussed in Section C.2 was developed by Nowak and Collins (2013) and was restricted to single limit states, as the Load and Resistance units and distributions vary between limit states. The procedure was based on the Rackwitz and Fiessler $(1976,1978)$ procedure for mapping a non-normal distribution to a normal distribution with unit area. This procedure works well with continuous data and less well with histogram data because the routine requires computation of matching cumulative distribution function (CDF) and PDF data for the distribution with the normal distribution. This procedure allows for the scaling of the resistance distribution with respect to a fixed load distribution, adjusting the $(R-L)$ distribution for a target value of $\beta$ and its associated PUP.

This Nowak and Collins (2013) numerical procedure was adapted for use in solving problems discussed in this report. It stands in contrast with the ASM procedure in that ASM determines a $\beta$ value that corresponds to the probability of unsatisfactory performance given fixed load and resistance distributions. Load and resistance factors are computed in the ASM 
approach from a design point that resides on the limit state response surface. These load and resistance factors guarantee the probability of unsatisfactory performance for the computed value for $\beta$.

To summarize, the ASM method takes resistance and load distributions and calculates a $\beta$ value for a limit state to compute load and resistance factors. In contrast, the Nowak and Collins (2013) numerical procedure iteratively scales the resistance distribution while constraining the load distribution as fixed in reliability space allowing for calculation of load and resistance factors for a specified $\beta$ value.

A supplemental procedure is discussed in Section C.3 that recognizes the fact that load and resistance factors are constants and therefore their ratio is constant. It is simpler to solve for this ratio of these two factors and then to calculate the partial load safety factor given a specified partial resistance safety factor. This safety factor ratio may be computed using the numerical procedure outlined in Section C.2.

Section C.5 summarizes a reliability-based numerical procedure for computing a value for Reliability Index $\beta$ following the steps outlined in Nowak (1999). Professor Nowak originally developed this procedure over a number of years with a focus on its application to LRFD-based bridge design (Nowak and Lind 1979; Nowak 1999; Nowak and Collins 2000, 2013).

Section C.6 describes a Gaussian function superposition approach of numerically fitting normalized normal distribution functions to a nonnormal PDF and computing a value for Reliability index $\beta$. This approach uses a number of summed Gaussian distribution values for each PDF value. The error between the non-normal distribution PDF and the summed Gaussian distributions PDF is minimized. The attractiveness of this analysis procedure is that it may be used on any form of PDF yet be able to determine a value for $\beta$ that may be used in a Reliability analysis for determination of load and resistance factors.

Chapter 2 describes the geotechnical example problem that can be solved with SSI analysis using the equations of Chapter 3 for a T-Wall. This solution method depends on the design of the structural system, with a standard design defining the normal operating conditions and therefore the Usual load conditions and the three variable geotechnical material 
properties of Mohr-Coulomb effective angle of internal friction for the retained soil wedge, the foundation and the resisting soil wedge, $\phi_{1}, \phi_{2}{ }_{2}$ and $\phi_{3}$, respectively. The three strength parameter variables for the three Figure 2-1 soil regions are assumed to be normally distributed, independent variables. Their statistical parameters are summarized in Table 2-1. The base width was varied, and the design was conducted with the USACE Computer-Aided Structural Engineering (CASE) CTWall software using allowable stress design (ASD) method applying the traditional engineering safety factors of EM 1110-2-25O2 (HQUSACE 1989) for the Usual load case for each of the three Section 3.1 geotechnical limit states. The maximum base width that met the allowable stress design was for the sliding limit state ( $F S$ against sliding equal to 1.5 for the Normal load case). The sliding limit state was found to be critical among the three limit states, with the other two being bearing and base area in compression. Recall from Table 3-1 in EM 1110-2-2104 (HQUSACE 2016) that LRFD factors are applied for the Extreme load case and are not used in the design for the Usual and Unusual load cases. The limit states for this geotechnicalstructural system are described in Section 1.5.

Given the design geometry as established using CTWall software for the Normal load case and the geotechnical material properties, the resulting limit state load and resistance are computed for the Extreme load case, and those values can be used to assess the performance of the structure in a probabilistic framework. A set of samples can be created by varying the material properties in a Monte Carlo style simulation. These sample points can be used to create a PDF histogram of load and resistance. It has been observed for this example T-Wall that the resulting distributions can be very different from a normal distribution. Recall $\phi_{1}^{\prime}, \phi_{2}{ }_{2}$ and $\phi^{\prime}{ }_{3}$ are each normally distributed. Because the load $L$ and resistance $R$ (defined in Chapter 3), are computed from the same three wedge EM 1110-2-2502 (HQUSACE 1989) based analysis formulation and geotechnical material properties, the load and resistance distributions have a great likelihood to be correlated.

\subsection{Individual limit state procedure for computing load and resistance distributions from material properties and determining load factors}

The following procedure provides a method based on Monte Carlo simulations with material variables for determination of LRFD load factors. This procedure was devised by Ebeling and White (2019) for the reliability 
analysis of a T-Wall retains earth and is buttressed by earth, analytically modeled as an EM 1110-2-2502 (HQUSACE 1989) three-wedge retaining structure, with driving, structural, and resisting wedges. The MohrCoulomb shear strength parameters of effective angles of internal friction $\left(\phi_{1}^{\prime}, \phi_{2}^{\prime}\right.$, and $\left.\phi_{3}^{\prime}\right)$ for the soils of the three respective wedges were assumed to be normally distributed, independent variables. A traditional Reliabilitybased (Resistance-Load, or $R-L$ ) formulation of loads applied to the structural wedge containing the T-Wall was devised. Monte Carlo simulations were conducted to develop a database of corresponding resistance and load values which were then binned into a histogram to form PDF distributions. The resulting $R$ and $L$ distributions were observed to be non-normal because of the limit state computation method to calculate $R$ and $L$ from the three-wedge solution. Furthermore, because the threewedge solution method was used to compute load and resistance relative to each other, the distributions exhibited correlation. Rather than attempt to sample the load and resistance distributions and try to match the Pearson (1895) correlation coefficient, the original captured samples of corresponding $\mathrm{L}$ and $\mathrm{R}$ are used to calculate a Load Scale Factor $\left(L_{S F}\right)$ from a Resistance Scale Factor $\left(R_{S F}\right)$. The $R_{S F}$ is the scale factor that must be applied to the resistance distribution determined from the base structural design in order to achieve a target $P U P$ value $\left(P U P_{\text {Target }}\right) . R_{S F}$ times the mean Resistance ( $\mu$ Resistance) determines the resistance required to counteract the design load, and so it is equivalent to the design load. The mean Load ( $\mu$ Load) must be scaled by $L_{S F}$ to get the design load value. Designing the new resistance to meet this design load means the newly designed structure will achieve PUPTarget. This means that $L_{\boldsymbol{S F}}$ is equivalent to the ratio of the load and resistance factors $\left(\gamma_{L} / \varphi_{R}\right)$. From this ratio, and given a specified $R_{S F}$ value, an $L_{S F}$ value can be computed using the routine described in Appendix C, Section C.3. This procedure will be referred to as the Single Limit State Simulation Fixed Load Procedure because the load distribution remains fixed while the resistance distribution is scaled.

1. Create $\mathrm{Gz}()$ function for the limit state(s) that returns total Resistance $(R)$, total Load $(L)$, and conditional value of unsatisfactory performance (where unsatisfactory performance occurs when $R-L<0.0$ ).

2. For geotechnical design, calculate the slip plane angle for the current design conditions and determine the geo-statistical variability of soil properties (e.g., Mohr-Coulomb effective angle of internal friction) for each soil wedge (Driving, Structural, Resisting). 
3. For a certain number of samples ( $\left.N_{\text {samples }}\right)$, generate simulation soil properties and collect $\mathrm{Gz}()$ results, maintaining the order of the results so that $R$ [index] corresponds to L[index]. Maintaining the order means that the distributions and any correlations are included in the sample data.

4. Calculate $\mu_{\text {Resistance }}$ and $\mu_{\text {Load }}$ for the $R[]$ and $L[]$ simulation results.

5. For verification, calculate the PUPInitial for the full simulation taking the number of simulations with unsatisfactory performance and dividing by the total number of simulations. This PUP Initial can be run through the inverse CDF function for a Normal Distribution to arrive at a somewhat arbitrary $\beta$ Initial value.

6. A $\beta$ target value for a normal distribution is given that can be used to determine PUPtarget.

7. Generate a function given $R[], L[], P U P_{\text {target }}$, as well as a scale factor for Resistance $R_{S F}$. This function calculates the unsatisfactory performance

$$
P U P_{R S F}=\frac{\sum_{i=1}^{N_{\text {samples }}}\left\{\left(R[i] * R_{S F}\right)-L[i]\right\}\left[\begin{array}{l}
0:>0.0 \\
1:<0.0
\end{array}\right.}{N_{\text {samples }}}
$$

To judge how close $P U P_{R S F}$ is to $P U P_{\text {target, }}$, the function returns a value $\left|P U P_{R S F}-P U P_{\text {target }}\right|$. This value is 0.0 when $P U P_{R S F}$ is equal to $P U P_{\text {target }}$ and greater than 0.0 otherwise.

8. Use a numerical procedure to find a value of $R_{S F}$ that minimizes the Step 7 function. Because the function returns a minimal value of 0.0 when $P U P_{R S F}$ is equal to PUP target, the resulting value of $R_{S F}$ is the scale factor from the original Resistance distribution $R[]$ to the Resistance distribution that, combined with the original Load distribution $L[]$ has the same PUP as PUPtarget.

9. The $R_{S F}$ value is a scale factor for the current design's Resistance Distribution that satisfies the PUP target goal. The $\mu_{\text {Resistance }}$ and $\mu_{\text {Load }}$ values are the means of the distributions that may be correlated; therefore, their values have the same level of correlation. This means that $L_{S F}$, which is the scale factor for the mean load that requires the scaled resistance, or

$$
\begin{gathered}
R_{S F} * \mu_{\text {Resistance }}-L_{S F} * \mu_{\text {Load }}=0.0 \\
L_{S F}=\frac{\mu_{\text {Resistance }} * R_{S F}}{\mu_{\text {Load }}}
\end{gathered}
$$


10. According to the Limit State equation, the mean Resistance to be designed for ( $\left.\mu_{\text {RDesign }}\right)$ is therefore

$$
\mu_{\text {RDesign }} \geq L_{S F} * \mu_{\text {Load }}
$$

The product of the load scale factor times the mean load value (i.e., the right-hand side in this equation) is viewed as the value of minimum mean capacity required to achieve the $P U P_{\text {target }}$ value. Thus, this minimal design resistance $R_{\text {Design }}$ is the exact minimum mean resistance required to achieve the PUPtarget capacity. Figures 4-1 and 4-2 show the effect of scaling the Resistance Distribution by a computed $R_{S F}$ for a specified PUPSystem. Notice that the Load Distribution is held constant as $R_{S F}$ is applied.

Figure 4-1. Unscaled Load and Resistance Distributions and the resulting Gz() distribution for the Sliding Limit State.

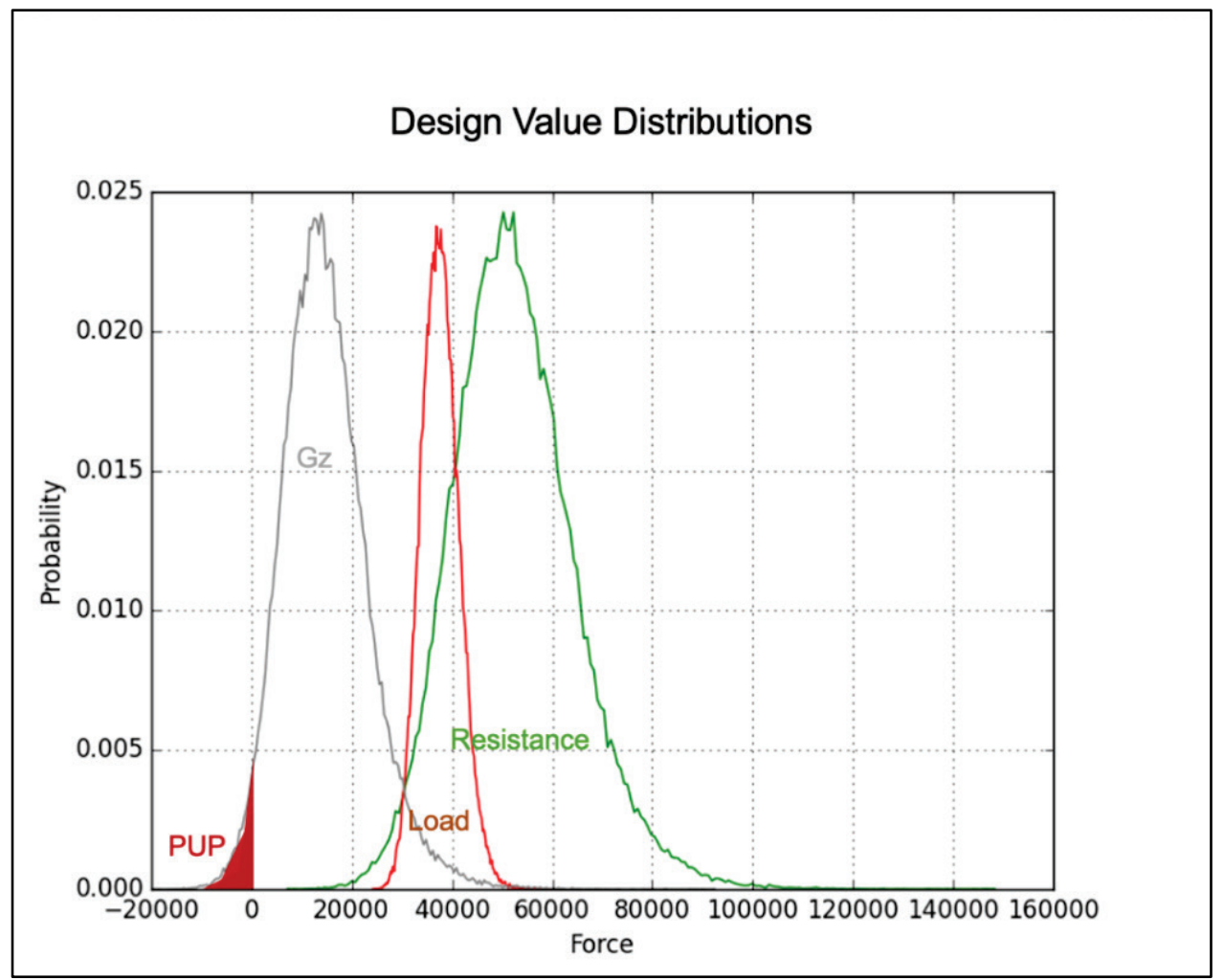


Figure 4-2. An $R_{S F}$ Scale Factor of 1.734 is applied to the Resistance Distributions and

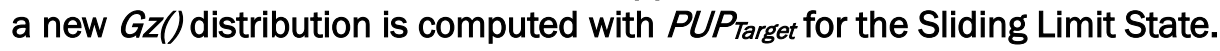

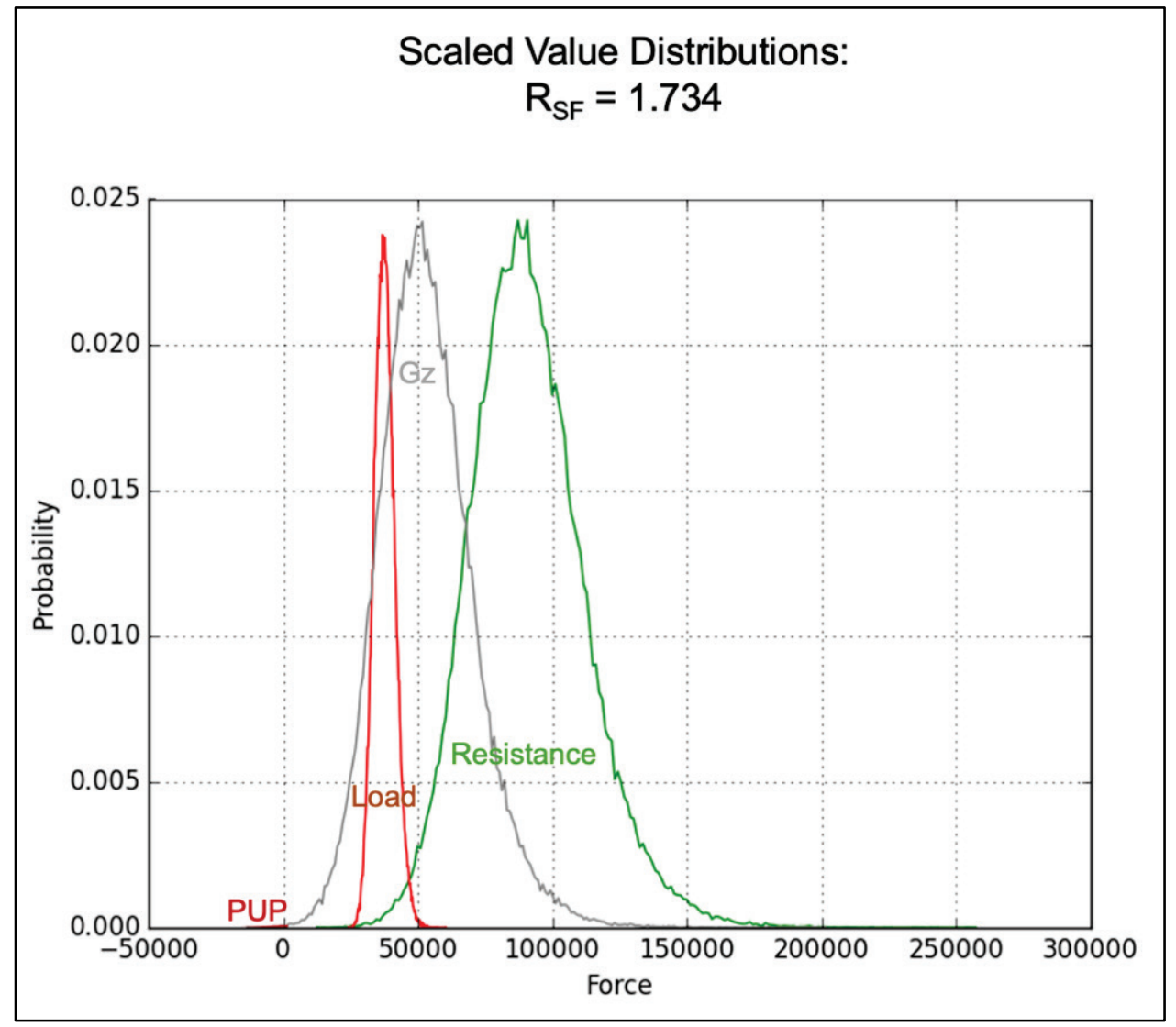

This procedure can be used for traditional LRFD, where the load and resistance distributions can be readily defined, although this is typically a trivial case that can be solved by basic analytical Reliability methods. This procedure becomes more important for issues where the resistance distribution can be calculated but is highly non-linear and/or is correlated to the load distribution. In this case, the procedure based on samples more accurately captures the behavior in the tails of the distributions, assuming that the design does not change the shape and dispersion rate of the distribution.

A full LRFD, with soil structure interaction, has more assumptions. In this case, the load distribution is not fixed but is a result of analysis of a design. The resistance distribution has these same characteristics and for the same reason. Each design case makes a difference in the mean loads and resistances because it also changes the forces acting on the structural wedge and therefore the T-Wall structure. Because the load conditions change the elevation of water and the slip planes of the driving and resisting Wedges, the mean Load and Resistance on the structure changes with the design. 
For the example problem, the Normal load case is described, with sloping backfill with an elevated internal water table within the retained soil, partial pool side fill, and a pool elevation. Three other load cases were considered (in order of severity). These cases may be considered as the Extreme design load case and two beyond-Extreme load cases for the structural system. The three cases are for the same T-wall but (1) dewatered on the pool side (Extreme), (2) dewatered with pool side fill being lowered to the top of the T-Wall foundation (beyond Extreme), and (3) dewatered with no fill on the former pool side (beyond Extreme). In each sequential case, there is less resisting soil and water to the backfill soil on the land side of the T-wall than existed in the prior case. These four load cases are illustrated in Figure 4-3.

Figure 4-3. Representations of the four design cases: (a) Normal Load Case, (b) Dewatered Load Case, (c) Dewatered with Reduced Fill for the Resisting Wedge, and (d) Dewatered with No Fill for the Resisting Wedge.

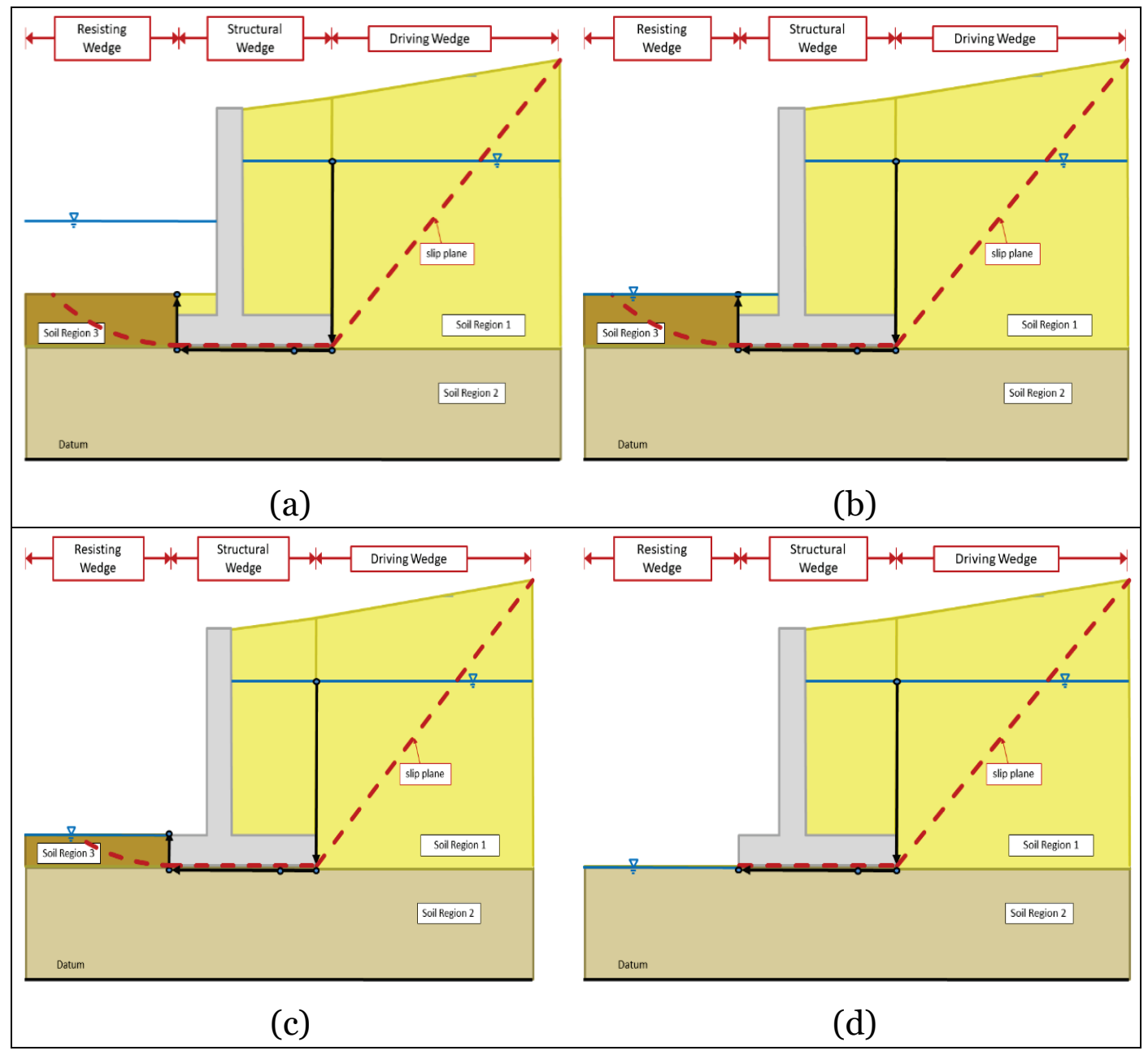




\subsection{Sliding Limit State results for mid-range COVs}

Tables 4-1 through 4-4 show the results of computing $R_{S F}$ and $L_{S F}$ for the Sliding Limit State load conditions (computed as forces) for a T-Wall designed with a $25 \mathrm{ft}$ base for different $P U P_{\text {target }}$ values based on specified normal distribution $\beta_{\text {target }}$ values. It was determined through a trial-anderror progression that setting $n_{\text {samples }}$ to 1,000,000 simulations would give sufficiently accurate results. Notice that each design load condition has a different $\mu_{R}$ and $\mu_{L}$ because the design load case affects the load and resistance. The $L_{S F}$ value is based on the current load condition's mean values, $\mu_{R}$ and $\mu_{L}$.

Table 4-1 is for the standard Normal load case of Figure 4-3a. This case is not traditionally solved for load factors for LRFD because the goal of LRFD for the USACE Navigation structures is to reduce the probability of Extreme load events causing unsatisfactory performance for the structural system as per EM 1110-2-2104 (HQUSACE 2016). This case is solved for the resistance and load factors here to provide insight into the full story of how load factors are developed for the Sliding geotechnical limit state problem. Solving this case also establishes the value of the Reliability Index, $\beta$, that the traditional EM 1110-2-2502 (HQUSACE 1989) allowable stress design procedure achieves given the Normal load case, which resulted in a $25 \mathrm{ft}$ wide base with an FS against sliding equal to 1.5. EM 1110-2-2100 (HQUSACE 2005) requires that the FS for sliding of a Normal structure using the Ordinary Site Information Category must meet or exceed 1.5 for the Usual load case. Monte Carlo simulation with material variables possessing Normal distributions was used to determine the PUP value for the normal load case design, resulting in a PUP of 0.00025 , which equates to a $\beta$ value of 3.48 (approximated with a Normal Gaussian Distribution). Using the Single Limit State Simulation Fixed Load Procedure (Subsection 4.1), the Table 4-1 values were produced. It is possible to interpolate the design $\beta$ using the target $\beta$ 's and $R_{S F}$ factors from Table 4-1, because the design $\beta$ occurs when $R_{S F}=1.0$ where the scale factor results in the values of the simulation being unchanged. Interpolating between the values of the last two rows gives a design $\beta$ of 3.48 , which matches the simulation value. This defines the performance of the $25 \mathrm{ft}$ wide $\mathrm{T}$-Wall designed with a sliding factor of safety of 1.5 for the Usual load case in a probabilistic framework. 
Table 4-1. For the $25 \mathrm{ft}$ wide base with Normal (watered) operating conditions: $\mu_{R}=54,042.96 \mathrm{lb}, \mu_{L}=38,963.16 \mathrm{lb}, \beta_{\text {initial }}=3.48, P U P_{\text {initial }}=0.00025, R[]$ to $L[]$ correlation $=0.976556$.

\begin{tabular}{|l|l|l|l|}
\hline \multirow{2}{*}{$\beta_{\text {target }}$} & PUP & Scale Factors & \\
\cline { 3 - 4 } & & $R_{S F}$ & $L_{S F}$ \\
\hline 1.0 & 0.15866 & 0.779 & 1.08 \\
\hline 1.5 & 0.06681 & 0.811 & 1.125 \\
\hline 2.0 & 0.02275 & 0.847 & 1.175 \\
\hline 2.5 & 0.00621 & 0.889 & 1.233 \\
\hline 3.0 & 0.00135 & 0.939 & 1.303 \\
\hline 3.5 & 0.00023 & 1.002 & 1.390 \\
\hline
\end{tabular}

Table 4-2. For the $25 \mathrm{ft}$ wide base with Extreme (dewatered) operating conditions: $\mu_{R}=51,763.52 \mathrm{lb}, \mu_{L}=37,574.50 \mathrm{lb}, \beta_{\text {initial }}=2.92, P U P_{\text {initial }}=0.00175, R[]$ to $L[]$ correlation $=0.978495$.

\begin{tabular}{|l|l|l|l|}
\hline \multirow{2}{*}{$\beta_{\text {target }}$} & PUPtarget & Scale Factors \\
\cline { 3 - 4 } & & $R_{S F}$ & LSF \\
\hline 1.0 & 0.15866 & 0.779 & 1.101 \\
\hline 1.5 & 0.06681 & 0.837 & 1.156 \\
\hline 2.0 & 0.02275 & 0.886 & 1.220 \\
\hline 2.5 & 0.00621 & 0.940 & 1.295 \\
\hline 3.0 & 0.00135 & 1.016 & 1.400 \\
\hline 3.5 & 0.00023 & 1.187 & 1.635 \\
\hline
\end{tabular}

Table 4-3. For the $25 \mathrm{ft}$ wide base with beyond-Extreme soil to top of base (dewatered) operating conditions:

$\mu_{R}=43,459.34 \mathrm{lb}, \mu_{L}=34,874.95 \mathrm{lb}, \beta_{\text {initial }}=1.39, P U P_{\text {initial }}=0.08229, R[]$ to $L[]$ correlation $=0.973397$.

\begin{tabular}{|l|l|l|l|}
\hline \multirow{2}{*}{$\beta_{\text {target }}$} & \multirow{2}{*}{ PUP target } & \multicolumn{2}{|l|}{ Scale Factors } \\
\cline { 3 - 4 } & & $R_{S F}$ & LSF \\
\hline 1.0 & 0.15866 & 0.938 & 1.169 \\
\hline 1.5 & 0.06681 & 1.025 & 1.277 \\
\hline 2.0 & 0.02275 & 1.191 & 1.484 \\
\hline 2.5 & 0.00621 & 1.437 & 1.790 \\
\hline 3.0 & 0.00135 & 1.810 & 2.255 \\
\hline 3.5 & 0.00023 & 2.246 & 2.798 \\
\hline
\end{tabular}


Table 4-4. For the $25 \mathrm{ft}$ wide base with beyond-Extreme scoured soil to the toe of the structure (dewatered) operating conditions:

$$
\begin{gathered}
\mu_{R}=42,775.71 \mathrm{lb}, \mu_{L}=34,427.89 \mathrm{lb}, \beta_{\text {initial }}=1.251, P U P_{\text {initial }}=0.10551, R[] \text { to } L[] \\
\text { correlation }=0.971258 \text {. }
\end{gathered}
$$

\begin{tabular}{|l|l|l|l|}
\hline \multirow{2}{*}{$\beta_{\text {target }}$} & \multirow{3}{|l|}{ SUP } & Scale Factors \\
\cline { 3 - 4 } & & $R_{S F}$ & LSF \\
\hline 1.0 & 0.15866 & 0.956 & 1.187 \\
\hline 1.5 & 0.06681 & 1.072 & 1.331 \\
\hline 2.0 & 0.02275 & 1.283 & 1.594 \\
\hline 2.5 & 0.00621 & 1.574 & 1.956 \\
\hline 3.0 & 0.00135 & 2.061 & 2.561 \\
\hline 3.5 & 0.00023 & 2.747 & 3.413 \\
\hline
\end{tabular}

For the example problem, Extreme conditions are met when the structure is dewatered, as depicted in Figure 4-3b. Flood-side soil erosion, as depicted in Figure 4-3c and d, are special beyond extreme load cases that are outside of the acceptable bounds for the structure. They are included to show scale factor growth as conditions change.

For the dewatered case using the design with a $25 \mathrm{ft}$ base width, the program CTWALL-R (Pace 1994) returned an FS for sliding of 1.43. The EM 1110-2-2100 (HQUSACE 2005) requires that the FS for sliding of a Normal structure using the Ordinary Site Information Category must meet or exceed 1.1 for the Extreme load case. Therefore, the designed structure has met the requirement by a significant amount, even exceeding the minimum Unusual load case safety factor of 1.3. Monte Carlo simulations of this design under the Extreme load case yields a $\beta_{\text {initial }}$ of 2.92, with a PUP initial of 0.00175. Table 4-2 gives the scale factors for the Extreme load case of Figure 4-3b. To achieve a PUPtarget of 0.00023 corresponding to a normal distribution $\beta$ target of 3.5, the load scale factor $L_{S F}$ is 1.635 giving a value of $R_{\text {Design }}$ equal to $61,434 \mathrm{lb}(=37,574.5$ times 1.635 by Equation 4.4$)$. This $61,434 \mathrm{lb}$ is viewed as the value of minimum mean capacity required to achieve the PUP target value, which should be designed for. Therefore, this value is also the minimal design resistance $R_{\text {Design. }}$ This procedure works if the load distribution is constant.

Tables 4-1 through 4-4 reveal that the mean load changes as the design is altered, going from a mean load of $54,043 \mathrm{lb}$ for the Normal load case to a mean load of 42,776 lb for the most Extreme load case. This implies that any redesign that has a resistance distribution with a mean that approaches 
a desired $R_{\text {Design }}$ value is also altering the load distribution, and therefore the PUP and $\beta$ for the system. This is because the solution method given a design computes the Load and Resistance from the mobilized soil regions via the slip plane computation of the three-wedge system. If the engineer is trying to achieve a design that exceeds a $P U P$ value precisely, a few design iterations in T-Wall base width may be required per load case. The extreme value of $L_{S F}$ given $\beta$ target 3.5 for Table 4-2 yields a value for $R_{\text {Design }}$ of $61,434 \mathrm{lb}$ (Equation 4.4), and that value appears to be a high value, given the other computed resistances for the $25 \mathrm{ft}$ base load cases.

Tables 4-1 through 4-4 also give the initial values for $\beta$ and PUP given the original computed distributions. These values corroborate the calculations for $R_{S F}$, as the value for $R_{S F}$ is less than 1.0 while $\beta_{\text {target }}$ is less than $\beta_{\text {initial }}$ and greater than 1.0 otherwise. The same holds true for the PUP values.

In the Tables 4-1 through 4-4 titles, the correlation values between the Resistance and Load distributions are reported for the Sliding load case. These distributions were found to have at least a $97 \%$ correlation between the sample resistance and load values. Other statistical tests were performed to determine the Pearson correlation coefficients between the input variables ( $\phi^{\prime} D W, \phi^{\prime} S W$, and $\left.\phi^{\prime}{ }_{R W}\right)$ to assure that the input variables were independent after their simulation. These variables had correlation coefficients between each (simulation) sample set with values less than 0.004, confirming each variable's independence from each other. This result means that any correlation introduced to the derived load and resistance distributions is not due to the simulation procedure. Because this procedural method works with direct simulation results, the distribution is preserved with its correlation directly when the resistance values are scaled. This is different than the Nowak and Collins (2013) procedure discussed in Appendix C, which relies on Resistance and Load distributions for its variables that are uncorrelated. The conversion of the load and resistance distributions using the Rackwitz-Fiessler $(1976,1978)$ transform is confounded by correlated variables.

In summary, the numerical Reliability simulation procedure outlined in Section 4.2 and applied to this $25 \mathrm{ft}$ base width T-Wall example is devised for computations made using a single limit state at a time because in this procedure the load and resistance distributions are calculated expressly for that limit state. 


\subsection{Base Area in Compression Limit State results for mid-range COVs}

Tables 4-5 and 4-6 show the results of computing $R_{S F}$ and $L_{S F}$ for the Base Area in Compression (i.e., Overturning) Limit State load conditions for a T-wall designed with a $25 \mathrm{ft}$ base for different $P U P_{\text {target }}$ values based on specified normal distribution $\beta$ target values. The resistance and load distributions are computed in units of moment, foot-pounds. Notice that each design load condition has a different $\mu_{R}$ and $\mu_{L}$ because the design load case affects the load and resistance. The $L_{S F}$ value is based on the current load condition's mean values, $\mu R$ and $\mu L$.

Table 4-5. For the $25 \mathrm{ft}$ wide base with standard (watered) operating conditions: $\mu_{R}=1,390,809.72 \mathrm{ft}-\mathrm{lb}, \mu_{L}=806,216.96 \mathrm{ft}-\mathrm{lb}, \beta_{\text {initial }}>6.0, P U P_{\text {initial }}=0.00000, R[]$ to $L[]$ correlation $=0.298669$.

\begin{tabular}{|l|l|l|l|}
\hline \multirow{2}{*}{$\beta_{\text {target }}$} & \multirow{3}{*}{ PUP } & \multicolumn{2}{l|}{ Scale Factors } \\
\cline { 3 - 4 } & & $R_{S F}$ & $L_{\text {SF }}$ \\
\hline 1.0 & 0.15866 & 0.586 & 1.010 \\
\hline 1.5 & 0.06681 & 0.589 & 1.015 \\
\hline 2.0 & 0.02275 & 0.592 & 1.021 \\
\hline 2.5 & 0.00621 & 0.595 & 1.026 \\
\hline 3.0 & 0.00135 & 0.598 & 1.031 \\
\hline 3.5 & 0.00023 & 0.601 & 1.037 \\
\hline
\end{tabular}

Table 4-6. For $25 \mathrm{ft}$ wide base with standard (dewatered) operating conditions: $\mu_{R}=1,370,070.13 \mathrm{ft}-\mathrm{lb}, \mu_{L}=740,131.49 \mathrm{ft}-\mathrm{lb}, \beta_{\text {initial }}>6.0, P U P_{\text {initial }}=0.00000, R[]$ to $L[]$ correlation $=0.331293$.

\begin{tabular}{|l|l|l|l|}
\hline \multirow{2}{*}{$\beta_{\text {target }}$} & \multirow{3}{*}{ PUP } & \multicolumn{2}{|l|}{ Scale Factors } \\
\cline { 3 - 4 } & & $R_{S F}$ & $L_{\text {SF }}$ \\
\hline 1.0 & 0.15866 & 0.546 & 1.011 \\
\hline 1.5 & 0.06681 & 0.549 & 1.017 \\
\hline 2.0 & 0.02275 & 0.553 & 1.023 \\
\hline 2.5 & 0.00621 & 0.556 & 1.029 \\
\hline 3.0 & 0.00135 & 0.559 & 1.035 \\
\hline 3.5 & 0.00023 & 0.562 & 1.040 \\
\hline
\end{tabular}


It may cause pause when observing that the $L_{S F}$ values for both load cases are greater than 1.o, when the resistance needs to be lowered to less than or equal to 0.6 of its mean value. This is because there is so much excess capacity for the design and $\mu_{R}$ is greater than 2 times the $\mu_{\mathrm{L}}$. Recall the design of this $25 \mathrm{ft}$ wide T-Wall is based on the Usual load case for the sliding limit state. It is speculated, based on the minimal scale change of load ( $\left.L_{S F}\right)$ to find the $R_{\text {Design }}$ value (by Equation 4.4), that the load and resistance distributions for this limit state possess small variance. This causes the slope of the PUP curve for the $\beta_{\text {target }}$ values to be steep and occur over a short span of base width values. This interpretation is bolstered by a series of runs varying the base width in the design between 12 and $15 \mathrm{ft}$ for the normal load case. In these runs, the approximate $P U P$ values decreased from 0.99 at the smaller base width of $12 \mathrm{ft}$ to a PUP value of 0.066 at base width of $13 \mathrm{ft}$, and continued to a value of 0.023 at $14 \mathrm{ft}$. At $15 \mathrm{ft}$ base width, the PUP value was essentially 0.o. Thus, for base width of $25 \mathrm{ft}$, the PUP value for the base area in compression limit state is o.o.

\subsection{Bearing Limit State results for mid-range COVs}

Tables 4-7 and 4-8 show the results of computing $R_{S F}$ and $L_{S F}$ for the Bearing Limit State load conditions for a T-wall designed with a $25 \mathrm{ft}$ base for different PUP $P_{\text {target }}$ values based on specified normal distribution $\beta_{\text {target }}$ values. The resistance and load distributions are computed in units of force, pounds. Notice that each design load condition has a different $\mu_{R}$ and $\mu_{L}$ because the design load case affects the load and resistance. This is being observed for all Geotechnical limit states. The $L_{S F}$ value is based on the current load condition's mean values, $\mu_{R}$ and $\mu_{\mathrm{L}}$.

Table 4-7. For the $25 \mathrm{ft}$ wide base with standard (watered) operating conditions:

$\mu_{R}=131,936.98 \mathrm{lb}, \mu_{L}=48,736.99 \mathrm{lb}, \beta_{\text {initial }}>6.0, P U P_{\text {initial }}=0.00000, R[]$ to $L[]$ correlation $=0.880871$.

\begin{tabular}{|l|l|l|l|}
\hline \multirow{3}{*}{$\beta_{\text {target }}$} & PUP & \multicolumn{3}{l|}{ Scale Factors } \\
\cline { 3 - 4 } & $R_{S F}$ & $L_{\text {SF }}$ \\
\hline 1.0 & 0.15866 & 0.401 & 1.085 \\
\hline 1.5 & 0.06681 & 0.417 & 1.129 \\
\hline 2.0 & 0.02275 & 0.436 & 1.180 \\
\hline 2.5 & 0.00621 & 0.460 & 1.245 \\
\hline 3.0 & 0.00135 & 0.506 & 1.370 \\
\hline 3.5 & 0.00023 & 0.561 & 1.518 \\
\hline
\end{tabular}


Table 4-8. For $25 \mathrm{ft}$ wide base with standard (dewatered) operating conditions: $\mu_{R}=149,225.00 \mathrm{lb}, \mu_{L}=54,808.31 \mathrm{lb}, \beta_{\text {initial }}>6.0, P U P_{\text {initial }}=0.00000, R[]$ to $L[]$ correlation $=0.811645$.

\begin{tabular}{|l|l|l|l|}
\hline \multirow{3}{*}{$\beta_{\text {target }}$} & PUP & Scale Factors & \\
\hline 1.0 & 0.15866 & $R_{S F}$ & LSF \\
\hline 1.5 & 0.06681 & 0.394 & 1.073 \\
\hline 2.0 & 0.02275 & 0.408 & 1.110 \\
\hline 2.5 & 0.00621 & 0.422 & 1.149 \\
\hline 3.0 & 0.00135 & 0.438 & 1.192 \\
\hline 3.5 & 0.00023 & 0.460 & 1.253 \\
\hline
\end{tabular}

The bearing capacity results have much larger resistance forces than for the sliding limit state because the forces are acting vertically and mobilizing more of the foundation soil. The higher resistances and smaller vertical loads lead to excess resistance. This causes the slope of the PUP curve for the $\beta$ target values to be steep and occur over a short span of base width values for the T-Wall, as was observed for the Base Area in Compression limit state.

\subsection{Soil Structure Interaction (SSI)-proportioned load individual limit state procedure for computing load and resistance distributions from material properties and determining load factors}

Subsection 4.2 introduces the first analytical simulation Reliability procedure used for load and resistance factors computation, and it is referred to as the Single Limit State Simulation Fixed Load Procedure because the resulting limit state load distribution for the testing structure remains fixed while the resistance distribution is scaled. Using simulation generated sliding limit state $R$ and $L$ data for T-Walls with base widths between $22 \mathrm{ft}$ and $28 \mathrm{ft}$, Figure 4-4 is introduced, which reveals that this Fixed Load Assumption, while valid for many engineering Reliability analysis, is not appropriate for the Figure 2-1 geotechnical soil structure interaction problem. The assumption breaks down because, for geotechnical problems of this type, a change of design (in this case a change in base width for the T-Wall) affects both the load and resistance forces from the interacting soil wedges. The sliding limit state $R$ and $L$ force simulation data are highly correlated for this T-Wall problem. An improved procedure, called the proportioned load procedure for predicting load and resistance factors, was then devised and applied to this same problem. This 
procedure adjusts the $L$ distribution as the $R$ distribution changes. The results from this second analysis are also included in this subsection. This procedure will be referred to as the Single Limit State Simulation Proportioned Load Procedure.

Figure 4-4 provides a visual comparison of simulation results versus the prediction results from using the Subsection 4.2 procedure. The orange solid line of the plot passes through data points collected for the simulation runs at each of the base widths from $22 \mathrm{ft}$ to $28 \mathrm{ft}$. The actual values of the simulations for these base widths return the resultant mean resisting force and the approximated Gaussian Normal distribution $\beta$ value for the simulated PUP value. The result from the unscaled simulated data is used to form the orange solid curved line segments and are judged to be precise. The dashed lines represent the predicted values from each base width's actual distributions of the mean resistance force given a target approximate Gaussian Normal $\beta$, or $\beta$ target. For example, with a $22 \mathrm{ft}$ base width run and a $\beta$ target of 3.0, the resistance force would be approximately 50,000 lb, by the intersection of the light blue dashed line with a $\beta$ target-value of 3.0 in this figure. The precise value for $\beta$ target of 3.0 from the orange direct simulated value line is a force of between 53,000 and $54,000 \mathrm{lb}$.

Figure 4-4. Predicted resistances (using the Section 4.2 procedure) for multiple wall widths to achieve a target beta (with fixed load) versus the actual $\beta$ values computed for each wall width.

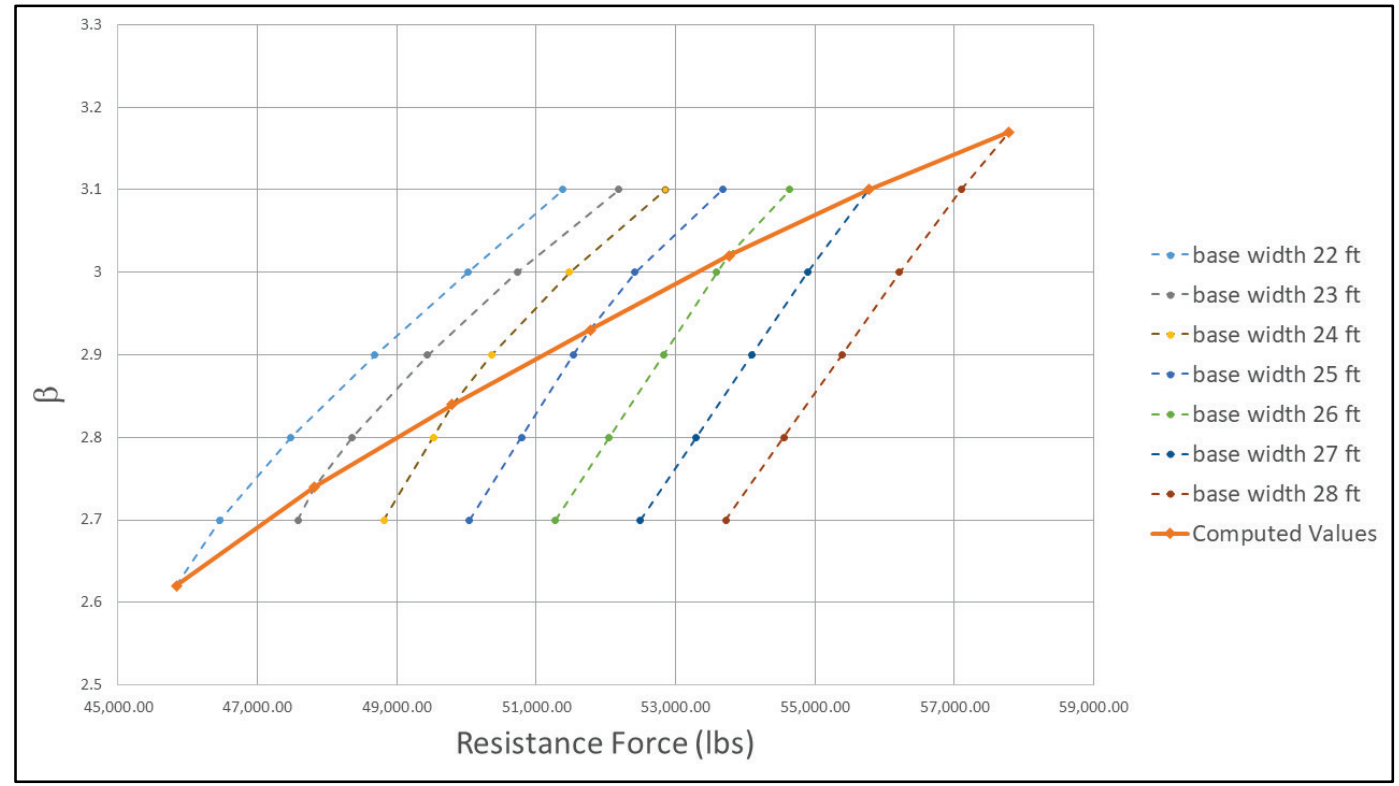


Note that while the slopes for individual predicted values (dashed lines) and the computed values are not linear, they do show linear tendencies, especially for the dashed line data that plots below the solid orange line. This suggests that it is possible to adjust the predicted values given load and resistance distributions for a single base width using the ratio of change of mean resistance to change of mean load as the base width changes, so long as the distributions about the means do not change significantly. In this way, a better prediction of $R_{S F}$ and therefore $L_{S F}$ can be computed given the load and current $\beta$ of a specific base width.

The following procedure provides a method based on Monte Carlo simulations with material definitions for determination of LRFD load factors. Rather than attempt to sample the load and resistance distributions and try to match the Pearson (1895) correlation coefficient, the original captured samples with computed resistance and load are used to calculate the Load Scale Factor $\left(L_{S F}\right)$ and Resistance Scale Factor $\left(R_{S F}\right)$. This procedure will be called the proportioned load procedure for predicting load and resistance factors.

1. Create $G z()$ function for the limit state(s) that returns total Resistance $(R)$, total Load $(L)$, and conditional value of unsatisfactory performance (where unsatisfactory performance occurs when $R-L<0.0$ )

2. For geotechnical design, calculate the slip angle for the current design conditions and determine the geo-statistical variability of soil properties (e.g., soil friction angle) for each soil wedge (Driving, Structural, Resisting)

3. Run a $\mathrm{Gz}()$ analysis for two different design cases (in this case, varying the base width), and collect the rate of change of $\mu_{\text {Load }}$ and $\mu_{\text {Resistance }}$ labeled $\Delta_{\text {Load }}$ and $\Delta$ Resistance, respectively. For greater accuracy, more runs can be made, and the rate of change values averaged.

4. For a certain number of samples ( $\left.N_{\text {samples }}\right)$, generate simulation soil properties and collect $\mathrm{Gz}()$ results for a base design case (e.g., single base width), maintaining the order of the results so that $R$ [index] corresponds to L[index]. Maintaining the order means that the distributions and any correlations are included in the sample data.

5. Calculate $\mu_{\text {Resistance }}$ and $\mu_{\text {Load }}$ for the $R[]$ and $L[]$ simulation results.

6. For verification, calculate the $P U P_{\text {Initial }}$ for the full simulation taking the number of simulations with unsatisfactory performance and dividing by the total number of simulations. This PUPInitial can be run through the 
inverse CDF function for a Normal Distribution to arrive at a somewhat arbitrary $\beta_{\text {Initial value. }}$

7. A $\beta$ target value for a normal distribution is given that can be used to determine PUPtarget.

8. Generate a function given $R[], L[], P U P_{\text {target, }}$ as well as a scale factor for Resistance $R_{S F}$. This function calculates the unsatisfactory performance

$$
P U P_{R S F}=\frac{\sum_{i=1}^{N_{\text {samples }}}\left\{\left(\begin{array}{c}
\left(R[i] * R_{S F}\right)- \\
\left.\left(L[i]+\left(\left(R_{S F}-1.0\right) * R[i]\right) * \frac{\Delta_{\text {Load }}}{\Delta_{\text {Resistance }}}\right)\right\}[0:>0.0 \\
1:<0.0
\end{array}\right.\right.}{N_{\text {samples }}}
$$

To judge how close $P U P_{R S F}$ is to $P U P_{\text {target, }}$ the function returns a value $\left|P U P_{R S F}-P U P_{\text {target }}\right|$. This value is 0.0 when $P U P_{R S F}$ is equal to $P U P_{\text {target }}$ and greater than 0.0 otherwise.

9. Use a numerical procedure to find a value of $R_{S F}$ that minimizes the Step 7 function. Because the function returns a minimal value of 0.0 when $P U P_{R S F}$ is equal to PUP target, the resulting value of $R_{S F}$ is the scale factor from the original Resistance distribution $R[]$ to the Resistance distribution that, combined with the original Load distribution $L[]$, has the same PUP as PUPtarget.

10. The $R_{S F}$ value is a scale factor for the current design's Resistance Distribution that satisfies the PUP target goal. The $\mu_{\text {Resistance }}$ and $\mu$ Load values are the means of the distributions that may be correlated; therefore, their values are have the same level of correlation. This means that $L_{S F}$, which is the scale factor for the mean load that requires the scaled resistance, or

$$
\begin{gathered}
R_{S f} * \mu_{\text {Resistance }}-L_{S F} * \mu_{\text {Load }}=0.0 \\
L_{S F}=\frac{\mu_{\text {Resistance }} * R_{S F}}{\mu_{\text {Load }}}
\end{gathered}
$$

11. According to the Limit State equation, the mean Resistance to be designed for $(\mu$ RDesign $)$ is therefore

$$
\mu_{R D e s i g n} \geq L_{S F} * \mu_{\text {Load }}
$$

The difference between this numerical routine and the one in Section 4.2 are in Steps 3 and 8, where the relationship of the change in load to resistance according to design changes is added to the computation in Step 8. 
Figure 4-5 shows the results of applying the proportioned load procedure for predicting load and resistance factors to estimate the resistance force necessary to achieve a $\beta$ target relating to an associated PUPtarget. Because simulations were run given base widths from 22 to $28 \mathrm{ft}$, average values could be computed for $\Delta_{\text {Load }}$ and $\Delta_{\text {Resistance, }}$ which were calculated as $729.6717 \mathrm{lbf}$ and 1,990.048 lbf, respectively.

Figure 4-5. Predicted resistances for multiple wall widths to achieve a target $\beta_{\text {target }}$ (with load adjusted to match the rate of change with the variable resistance) versus the actual $\beta$ values computed for each wall width.

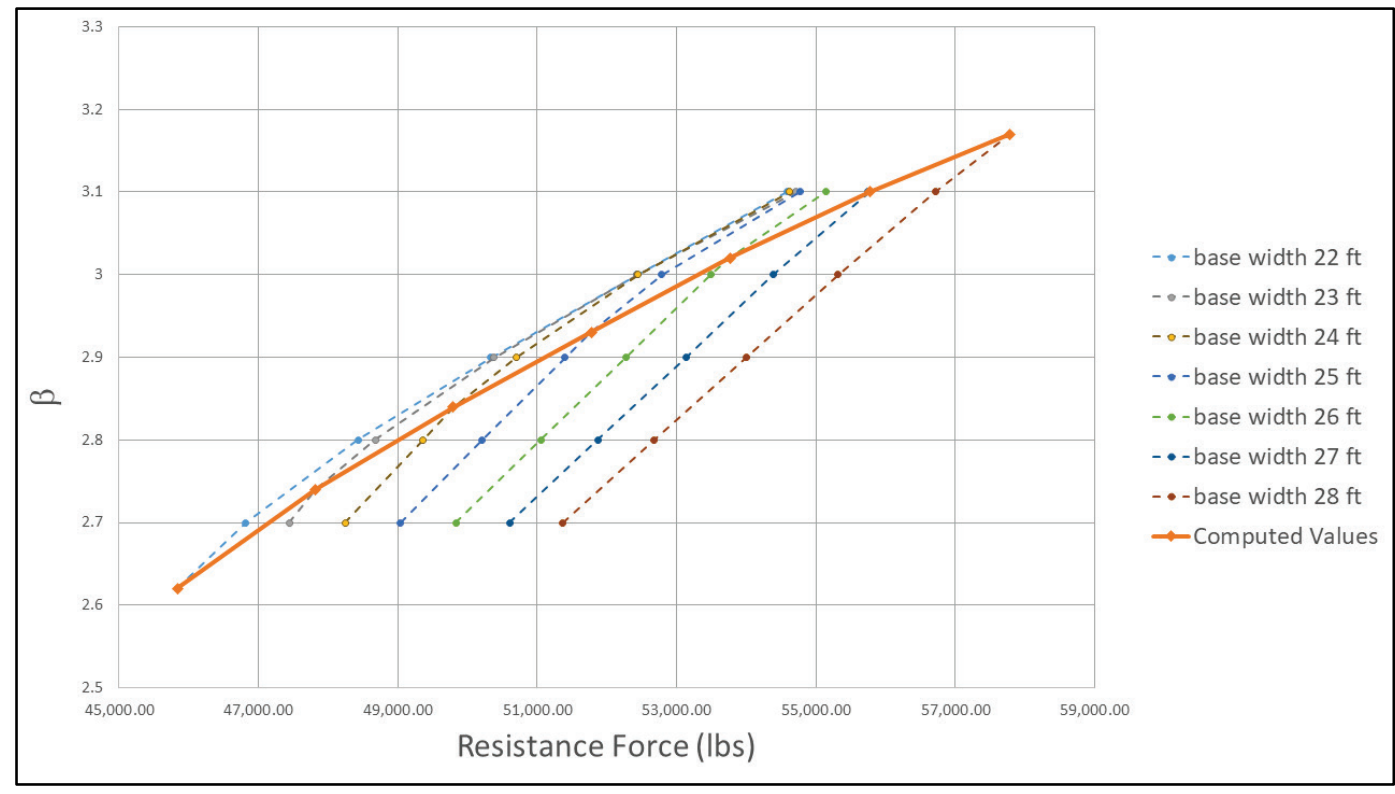

The results of using this procedure show an all-around improvement, but a much better improvement for higher values of $\beta_{\text {target }}$. The predicted values over all the base widths for $\beta_{\text {target }}=3.1$ range from $52,189 \mathrm{lbf}$ to $57,102 \mathrm{lbf}$ with a difference of nearly $5,000 \mathrm{lbf}$ using the fixed load procedure. The proportional load procedure has predicted values that range from $54,580 \mathrm{lbf}$ to $56,711 \mathrm{lbf}$ with a difference of just over $2,000 \mathrm{lbf}$. The computed actual value was 55,742 lbf, which nearly centers the smaller range.

For $\beta$ target equal to 2.7, the fixed load procedure predicted forces varied from $46,463 \mathrm{lb}$ to $53,718 \mathrm{lb}$. The proportional load predicted forces varied from $46,820 \mathrm{lb}$ to $51,366 \mathrm{lb}$. The precise value is approximately $47,158 \mathrm{lb}$, a value that is interpolated from the orange curve as it crosses the $\beta=2.7$ value, which is much nearer to the predicted values using the shorter base width.

Figure 4-6 shows the relationship of the predicted Resistance, Load, and (Resistance-Load) curves for direct simulations and simulation predictions 
using the proportioned load procedure. For the Resistance and Load curves, the $22 \mathrm{ft}$ base with T-Wall predicted histograms lie to the left of the actual $25 \mathrm{ft}$ histogram values and have a narrower range. The $28 \mathrm{ft}$ predicted Resistance and Load histograms lie to the right of the actual $25 \mathrm{ft}$ histogram values and have a wider range.

Subtracting the Loads from the Resistances, the $(R-L)$ histogram curves cross the 0.0 vertical axis, and PUP values are computed by integrating the values to the left of the o.o vertical axis. Note that the PUP values for the $25 \mathrm{ft}$ actual simulations and the 22 and $28 \mathrm{ft}$ predicted simulations are approximately the same; it is the mean values $\left(\mu_{R-L}\right)$ and standard distributions $(\sigma R-L)$ that change due the spread of the distribution values. Because less earth is mobilized with a shorter model base width, the spread of the distribution is less, and thus it is a better distribution to predict with. Recall that the proportioned load procedure prediction is based on mean loads and resistances.

Figure 4-6. Actual versus Predicted Resistance, Load, and R-L histograms for the $\beta_{\text {target }}$ value of 2.9348 corresponding to the actual $\beta$ for the $25 \mathrm{ft}$ base width model.

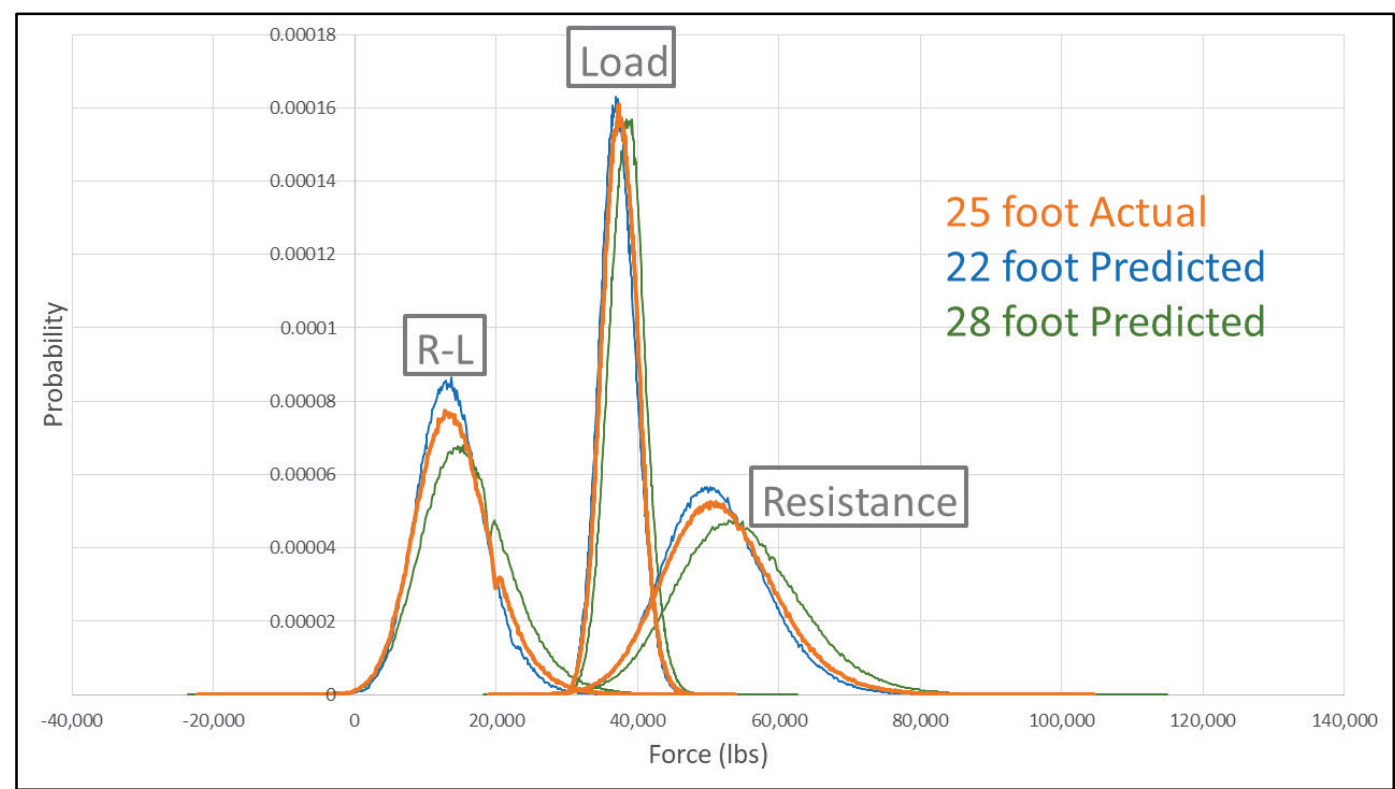

The proportioned load procedure yields better predictions when the $L$ and $R$ distributions have less dispersion (Figure 4-6). This is revealed by the data in Figure 4-5, as described subsequently: the $25 \mathrm{ft}$ base width precise (orange line) $\beta$ value is 2.93 and occurs with $\mu$ Resistance equal to $51,777 \mathrm{lb}$. The predictions from the base widths with precise $\beta$ values less than 2.93, which are the base widths less than $25 \mathrm{ft}$ for this example, are better than 
the base widths that have higher precise $\beta$ values. For example, the $22 \mathrm{ft}$ base width prediction for $\beta$ equal to 2.93 of the resistance force is approximately $51,066 \mathrm{lb}$. This has a liberal error of less than $2 \%$. Using the same example, the $28 \mathrm{ft}$ base width prediction for $\beta$ equal to 2.93 of the resistance force is approximately $54,470 \mathrm{lb}$. This has a conservative error of greater than $5 \%$. This trend is shown in Figure $4-5$ by the fact that the predicted curves (in dashed lines) that are above the orange solid line precise values more closely follow the precise line curvature, whereas the predicted curves follow a nearly steeper curvature below the precise curve.

For the proportioned load procedure to have a more accurate slope for the rate of change of $\mu$ Resistance to $\mu \mathrm{Load}$, at least a pair of design values that have precise $\beta$ values should encompass the $\beta_{\text {target }}$. The difference between the precise value $\mu_{\text {Resistance }}$ to $\mu_{\text {Load }}$ for the encompassing values provide the rate

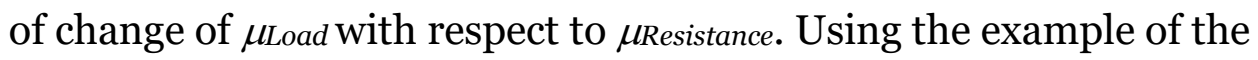
previous paragraph, the $28 \mathrm{ft}$ base width design, with $\beta$ equal to 3.17 , has

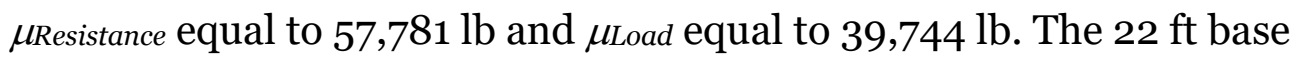
width design, with $\beta$ equal to 2.62 , has $\mu$ Resistance equal to $45,841 \mathrm{lb}$ and $\mu$ Load equal to $35,366 \mathrm{lb}$. Using these two values, the mean load changes at a rate of $4,378 \mathrm{lb}$ for each mean resistance change of $11,940 \mathrm{lb}$. This linear rate-ofchange slope provides the backbone for step 8 in the proportional load procedure. This allows a search for the mean resistance while maintaining the correlation-induced separation between $\mu_{\text {Resistance }}$ and $\mu_{\text {Load. }}$. These values are computed in Step 3 and applied in Step 8 of the proportional load procedure.

\subsection{Using $R_{S F}$ and $L_{S F}$ to compute load factor $\left(\gamma_{L}\right)$ and resistance factor $\left(\varphi_{R}\right)$}

Simulation values based on geospatial properties are used to determine the PUP initial for a single limit state of the current geotechnical design given the example T-Wall structure, initially with a base width of $25 \mathrm{ft}$. The value for $P U P_{\text {initial }}$ establishes the base relationship of $R_{S F}$ to $P U P_{\text {target }}$, When $P U P_{\text {target }}$ is equal to PUP initial, $R_{S F}$ is equal to 1 . The statistical dispersion of the simulation samples establishes the rate at which $R_{S F}$ changes as the current $P U P$ is adjusted to reach $P U P_{\text {target. }}$. In statistical simulations the population of simulated samples falling within the tails of the distribution are a critical feature affecting the accuracy of the PUP computation. Therefore, sufficient samples are required to achieve convergence as the 
current $P U P$ approaches $P U P_{\text {target. }} \varphi_{R}$ is sometimes referred to as $\gamma_{R}$ in this report, as $\gamma$ is often used to reference factors for forces.

$$
\varphi_{R} * \mu_{\text {Resistance }} \geq \gamma_{L} * \mu_{\text {Load }}
$$

$L_{S F}$ is the ratio of scaled mean resistance to design mean load, when $R_{S F}$ has been applied to the resistance samples to obtain PUPtarget (Equation 4.5). Multiplying the mean load times $L_{S F}$ results in the amount of resistance that must be designed for (designated $R_{\text {Design }}$ in Equation 4.4) to have a probability of unsatisfactory performance of $P U P_{\text {target }}$, if all conditions remain the same.

The resulting value of $L_{S F}$ times the mean load being required to be less than or equal to $R_{\text {Design }}$ (Equation 4.4) means that $L_{S F}$ is equivalent to the ratio of the load and resistance factors $\left(\gamma_{L} / \varphi_{R}\right)$, according to Equation 4.3 . Equation C.19 in Appendix C.3 (which transposes $\gamma_{\boldsymbol{R}}$ for $\varphi_{\boldsymbol{R}}$ ) reveals that, to achieve $P U P_{\text {target }}$ with varying resistances and loads, Equation 4.5 must be true for (1.0-PUP target), according to the Reliability Response Surface equation $G z()=0$.

\subsubsection{Computing load and resistance factors from $L_{s F}$}

Until this point, the load factor $\left(\gamma_{L}\right)$ and resistance factor $\left(\varphi_{R}\right)$ have not been considered separately, but as the ratio, $L_{S F}$. To determine the individual values of the two factors, a value for one of these factors has to be assigned and the other computed. It is important to realize that these two factors, by definition, are defined due to the uncertainty in the definition of the load and resistance variables combined with an acceptable $P U P_{\text {Target }}$ for the distribution of the difference in the resistance vector minus load vector of simulated values (i.e., $R[]$ minus $L[]$. The USACE has traditionally specified (e.g., in ASD) acceptable resistance capacity limitations expressed in terms of FS or its inverse, the SMF, applied to capacity term for the Geotechnical limit states (EM 1110-2-2502) (HQUSACE 1989). The FS/SMF terms have been tempered to allow for conservative computation of design strength given experience in the uncertainty of estimation. Therefore, the authors of this report suggest specifying a value for the resistance factor $\varphi_{R}$.

In consideration of the previous paragraph's discussion, it is therefore deduced that $R_{\text {Design }}$ has the higher variability, with negative impact when the resistance is less than that expected. This is reflected by values of $\varphi_{R}$ 
less than 1.o, reducing the amount of $R_{\text {Design. }}$ A resistance factor value of $\varphi_{R}$ less than 1.0 is cited in the Fenton et al. (2016) description of the Reliability-based Canadian Bridge design code that relates to geotechnical limit states.

In EM 1110-2-2104 (HQUSACE 2016) Table 3-1, the values of $\varphi_{R}$ applied to resistance forces determined by lateral earth pressures are 0.9 for at-rest conditions and 0.5 for all other conditions for the Extreme load case. The authors of this report suspect that this reflects the dispersion in soil strength properties for those Geotechnical limit state conditions. Recall that lateral earth pressures are important for calculating the Sliding and Base Area in Compression limit states. Once $\varphi_{R}$ has been assigned, Equation 4.6 uses the definition of $L_{S F}$ to determine $\gamma_{L}$.

$$
\gamma_{L}=\varphi_{R} * L_{S F}
$$

Using the Example problem Extreme load case values from Table 4-2, $L_{S F}=1.635$ when $\beta$ target $=3.5$ and $P U P_{\text {target }}=0.00023$. Under Extreme Load Case conditions, according to EM 1110-2-2104 (HQUSACE 2016), the value for $\varphi_{R}$ is 0.5 for the resisting force of the passive earth pressure distribution force. Using Equation 4.6, $\gamma_{L}$ would result in a value of 0.818 , and from this same table, $L_{S F}=1.4$ when $\beta_{\text {target }}=3.0$ and $P U P_{\text {target }}=0.000125$. Using Equation 4.6 with a $\varphi_{R}$ equal to 0.5 results in $\gamma_{L}$ equal to 0.7 . In both of these cases, applying $\varphi_{R}$ as 0.5 reduces the resistance distribution, which in turn causes the value of $\gamma_{L}$ to converge to a smaller value which is less than 1.o. This leads the authors of this report to question if the value of 0.5 is too conservative for $\varphi_{R}$. If the philosophy is taken that the factored load case should never be diminished, then the minimum $\gamma_{L}$ would be 1.o. Applying this value with $L_{S F}=1.4$, then the lowest value for $\varphi_{R}$ should be 0.71 by Equation 4.6.

Table 4-9 shows the results of performing the proportional method Monte Carlo simulations with the Sliding Limit state to determine the intermediate scale factors, $R_{S F}$ and $L_{S F}$, and then converting them to the load and resistance factors, $\gamma_{L}$ and $\phi_{R}$, for the defined Extreme load cases of Section 4.3. The load factors in this table increase as the Extreme load case conditions become more severe (e.g., reduction of soil). This indicates to the authors of this report that the selection of the Extreme Load case conditions and geometry can make a significant impact in the design of 
USACE hydraulic structures and lead to different Load (and when varied, Resistance) Factors.

Table 4-9. Computation of Load and Resistance scale factors with $\beta_{\text {target }}$ equal to 3.5 for each of the Extreme load conditions.

\begin{tabular}{|l|l|l|l|l|}
\hline $\begin{array}{l}\text { Extreme } \\
\text { Load Case }\end{array}$ & $R_{S F}$ & $L_{S F}$ & $\gamma_{L}$ & $\begin{array}{l}\phi_{R} \\
\text { (EM } \\
1110-2- \\
\text { 2104) } \\
\text { (HQUSACE } \\
\text { 2016) }\end{array}$ \\
\hline $\begin{array}{l}\text { Dewatered } \\
\text { Dewatered } \\
\text { Soil at } \\
\text { Base }\end{array}$ & 1.17 & 1.607 & 0.804 & 0.5 \\
\hline $\begin{array}{l}\text { Dewatered } \\
\text { with } \\
\text { Scoured } \\
\text { Soil }\end{array}$ & 2.74 & 2.766 & 1.383 & 0.5 \\
\hline
\end{tabular}

\subsubsection{Adjusting load and resistance factors to account for bias with nominal loads and resistances}

The procedure outlined for generating load and resistance factors in Section 4.7.1 works for the example problem, where load and resistance distributions are created as a result of computation applied to material variables drawn from well-defined distributions. These variables were the effective angles of internal friction for the Driving, Structural, and Resisting Wedges ( $\phi^{\prime}{ }_{D W}, \phi_{S W}^{\prime}$, and $\phi_{R W}^{\prime}$, respectively) and the computed length of the slip planes. Because the load and resistance distributions were computed directly from these values, there was no estimation bias in the distributions.

Bias factors are introduced due to the conservatism or liberalism in devised engineering computational procedures used to establish nominal resistance and load values. Subsection 2.1.2 in Ebeling and White (2019) discusses an example computation of a bias factor for the resistance of a pile foundation. The reader is referred to this subsection for further details on one approach that is used for computing a value for the bias factor.

Nominal distributions based on devised engineering design methodologies have two governing statistical components, a nominal mean value ( $\mu_{\text {nominal }}$ ) and the $\mathrm{COV}$, from which the standard deviation ( $\left.\sigma_{\text {nominal }}\right)$ can be obtained. 
The bias scale factor $(\lambda)$ is applied to $\mu_{\text {nominal }}$ to create the base mean value $(\mu)$. The COV is not changed and maintains the same relationship between the $\mu$ nominal and $\sigma_{n o m i n a l}$ as between $\mu$ and $\sigma$. This applies for both load and resistance distributions. From this point forward, the distribution with nominal values will be referred to in this report as the nominal distribution and the distribution with bias factors applied will be referred to as the mean distribution. Therefore, the means of the load and resistance distributions are

$$
\mu_{L}=\lambda_{L} * \mu_{\text {nominal }}
$$

and

$$
\mu_{R}=\lambda_{R} * \mu_{\text {nominal }}
$$

Applying these equations to the performance function inequality implied by Equation 4.2, which works with mean values:

$$
\varphi_{R} * \lambda_{R} * \mu_{\text {nominal }} \geq \gamma_{L} * \lambda_{L} * \mu_{\text {nominall }}
$$

The procedures that have been developed to find the $L_{S F}$ that guarantees a PUP target works with the mean distributions according to the $G z()$ functional Equations 4.2 and 4.3. Equation 4.4 gives the equation for the mean design resistance from $L_{S F}$ and $\mu_{\mathrm{L}}$. These leads to the following derivations:

$$
\begin{gathered}
\mu_{R}=L_{S F} * \mu_{L} \\
\lambda_{R} * \mu_{\text {nominalR }}=L_{S F} * \lambda_{L} * \mu_{\text {nominal } L}
\end{gathered}
$$

and

$$
\mu_{\text {nominalR }}=\frac{L_{S F} * \lambda_{L}}{\lambda_{R}} * \mu_{\text {nominalL }}
$$

This suggests that a nominal load scale factor LSFnominal can be introduced that scales $\mu$ nominalL to the design value of $\mu$ nominalR.

$$
\mu_{\text {nominalR }}=L_{S F n o m i n a l} * \mu_{\text {nominalL }}
$$


where

$$
L_{S F n o m i n a l}=\frac{L_{S F} * \lambda_{L}}{\lambda_{R}}
$$

Since the bias factors must be defined for the problem and the $L_{S F}$ value can be computed procedurally, LSFnominal can be found for the system.

From Equation 4.6,

$$
L_{S F}=\frac{\gamma_{L}}{\varphi_{R}}
$$

Substituting into 4.14,

$$
L_{S F n o m i n a l}=\frac{\gamma_{L} * \lambda_{L}}{\varphi_{R} * \lambda_{R}}
$$

Solving for $\gamma_{\mathrm{L}}$,

$$
\gamma_{L}=L_{\text {SFnominal }} * \frac{\varphi_{R} * \lambda_{R}}{\lambda_{L}}
$$

Using the EM 1110-2-2104 (HQUSACE 2016) Table 3-1 values as an unbiased $\varphi_{R}$ in the same manner mentioned at the beginning of Section 4.7, the value of the unbiased $\gamma_{L}$ can be determined. This is the generalized form of the load and resistance factors for nominal values with bias. If $\lambda_{L}$ and $\lambda_{R}$ are both 1 , then the equations revert to the form discussed in the previous sections.

\subsection{Using upper range point estimate coefficient of variation (COV) values for material properties}

Increasing the $\mathrm{COV}$ of the input material variables (e.g., effective angle of internal friction) for this system should increase the variability of the load and resistance for the model. These load and resistance variabilities acting on the Structural Wedge, in turn, will affect the generation of load and resistance factors. The values from Table 2-1 do not use the Upper Range values for $\mathrm{COV}$ for the effective angle of internal friction for example problem displayed in Figure 2-1.

The Upper Range point-estimate COV values for the effective angle of internal friction of the three soil regions are listed in Table 4-10 and based on 
the soils strength data contained in Appendix D of Ebeling and White (2019). These point-estimate COV values were converted to spatial COV values using the procedure outlined in Ebeling and White (2019) for use in the analysis summarized in this section. Table 4-10 lists these Upper Range COV values along with the original, Mid-Range COV values for comparison purposes.

Table 4-10. Engineering properties with Upper Range COV values for the three soil regions of the example T-Wall problem.

\begin{tabular}{|c|c|c|c|c|c|c|c|c|c|c|}
\hline \multirow[b]{2}{*}{$\begin{array}{c}\text { Soil } \\
\text { Region }\end{array}$} & \multirow[b]{2}{*}{ Soil Type } & \multicolumn{2}{|c|}{$\begin{array}{c}\text { Unit } \\
\text { Weights }\end{array}$} & \multicolumn{3}{|c|}{ Soil Friction, $\phi^{\prime}$} & \multicolumn{2}{|c|}{$\begin{array}{l}\text { Interface } \\
\text { Friction, } \delta^{\prime}\end{array}$} & \multicolumn{2}{|c|}{$\begin{array}{l}\text { Hydraulic } \\
\text { Conductivity }\end{array}$} \\
\hline & & $\begin{array}{l}\gamma_{\text {moist }} \\
\text { (pcf) }\end{array}$ & $\begin{array}{r}\gamma_{\text {sat }} \\
\text { (pcf) }\end{array}$ & $\begin{array}{c}\mu_{\phi^{\prime}} \\
\text { (deg) }\end{array}$ & $\begin{array}{c}\text { Mid- } \\
\text { Range } \\
\operatorname{cov}_{\phi^{\prime}} \\
(\%)\end{array}$ & $\begin{array}{c}\text { Upper } \\
\text { Range } \\
\text { COV }_{\phi} \\
(\%)\end{array}$ & $\begin{array}{l}\text { Ratio } \\
\delta^{\prime} / \phi^{\prime}\end{array}$ & $\begin{array}{c}\mu_{\delta} \\
(\mathrm{deg})\end{array}$ & $\begin{array}{c}\mu_{k} \\
(\mathrm{~cm} / \mathrm{sec})\end{array}$ & Direction \\
\hline 1 & $\begin{array}{c}\text { Compacted } \\
\text { sand }\end{array}$ & 123 & 126 & 35 & 10 & 20 & 0.4 & 14 & $2 \times 10^{-3}$ & Vertical \\
\hline 2 & $\begin{array}{l}\text { Silty sand } \\
\text { foundation }\end{array}$ & - & 123 & 30 & 20 & 30 & 1.0 & 30 & $4 \times 10^{-4}$ & Horizontal \\
\hline 3 & $\begin{array}{c}\text { Compacted } \\
\text { sand }\end{array}$ & - & 126 & 37 & 12 & 24 & 1.0 & 37 & $1 \times 10^{-3}$ & Vertical \\
\hline
\end{tabular}

To measure the effect of changing the COVs for the material variables ( $\phi^{\prime} D W$, $\phi_{S W}^{\prime}$, and $\phi_{R W}^{\prime}$ ), simulations were performed as the COVs were linearly changed from the original, Mid-Range COVs to the Upper Range COVs as characterized by the parameter $t$. The parameter $t$ is 0.0 for the original, Mid-Range COVs, 1.0 for the Upper Range COVs, and at select, linear interpolation for values for $t$ greater than 0.0 and less than 1.o. The procedure of Section 4.6 was performed with differing values of $\beta$ Target.

The relationship of Sliding Limit State $L_{S F}$ values to the change of COVs is given in Figure 4-7 for different values of $\beta_{\text {Target. }}$ The value of $\beta_{\text {Target, }}$, designated $B$ in this figure, range in value from a low of 1.0 to a high of 3.5. Because an increasing COV can be construed as increasing uncertainty about the mean values for the soil strength variables, the results indicate that the ratio of Load Factor to Resistance Factor increases as the dispersion increases. This makes sense, as the Load and Resistance Factors are generated to address uncertainty. 
Figure 4-7. Computed values for Sliding Limit State $L_{S F}$ with the same target $\beta$ (designated B) as the COVs go from the original, Mid-Range value $(t=0.0)$ to the Upper Range values $(t=1.0)$.

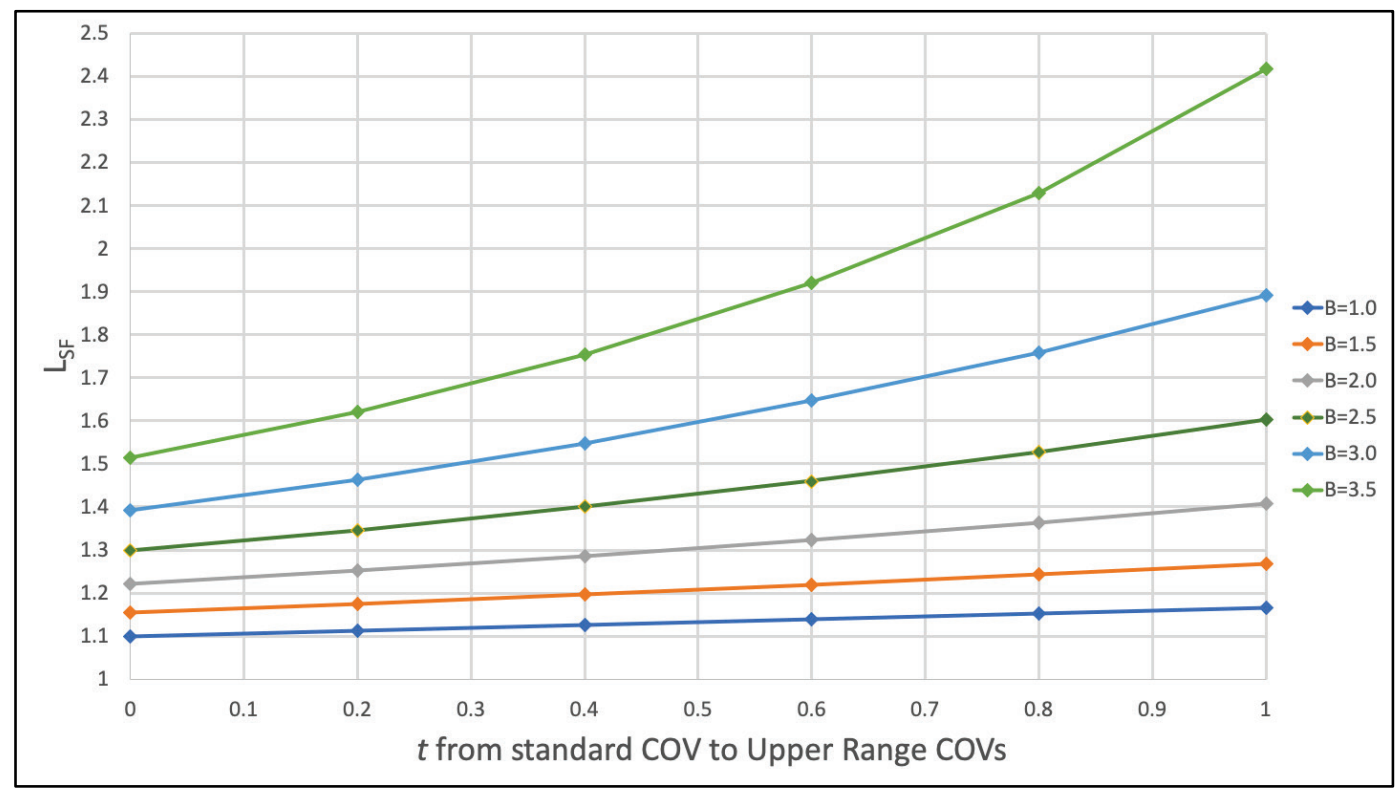

For the Figure 4-7 family of curves with different $\beta$ Target values, the rate of change in $L_{S F}$ values with increasing Upper Range COV values distances between consecutive curves increases and is observed to be increasingly non-linear for the largest COV values region (i.e., near $t=1.0$ ). This is to be expected as higher $\beta$ Target values approach the tails of the distributions, where sample density decreases.

The increase in the $L_{S F}$ values is not linear for any $\beta_{\text {Target }}$ value. The authors speculate that this non-linearity results because the computed Load distribution sees a greater increase in COV than the computed Resistance distribution.

Using Equation 4.6 with a Resistance Factor of 0.5, from EM 1110-2-2104 (HQUSACE 2016) Table 3-1 Extreme load case lateral earth pressures, with the results in Figure 4-7 gives the Load Factor to uncertainty plot of Figure 4-8. The relationship of Equation 4.6 demonstrates that for a Resistance Factor held constant, the Load Factor increases in value as dispersion/uncertainty increases. 
Figure 4-8. Computed values for Sliding Limit State Load Factor (based on a resistance factor of 0.5 ) with the same target $\beta(B)$ as the COVs go from the original, Mid-Range value ( $t=0.0$ ) to the Upper Range values ( $t=1.0)$.

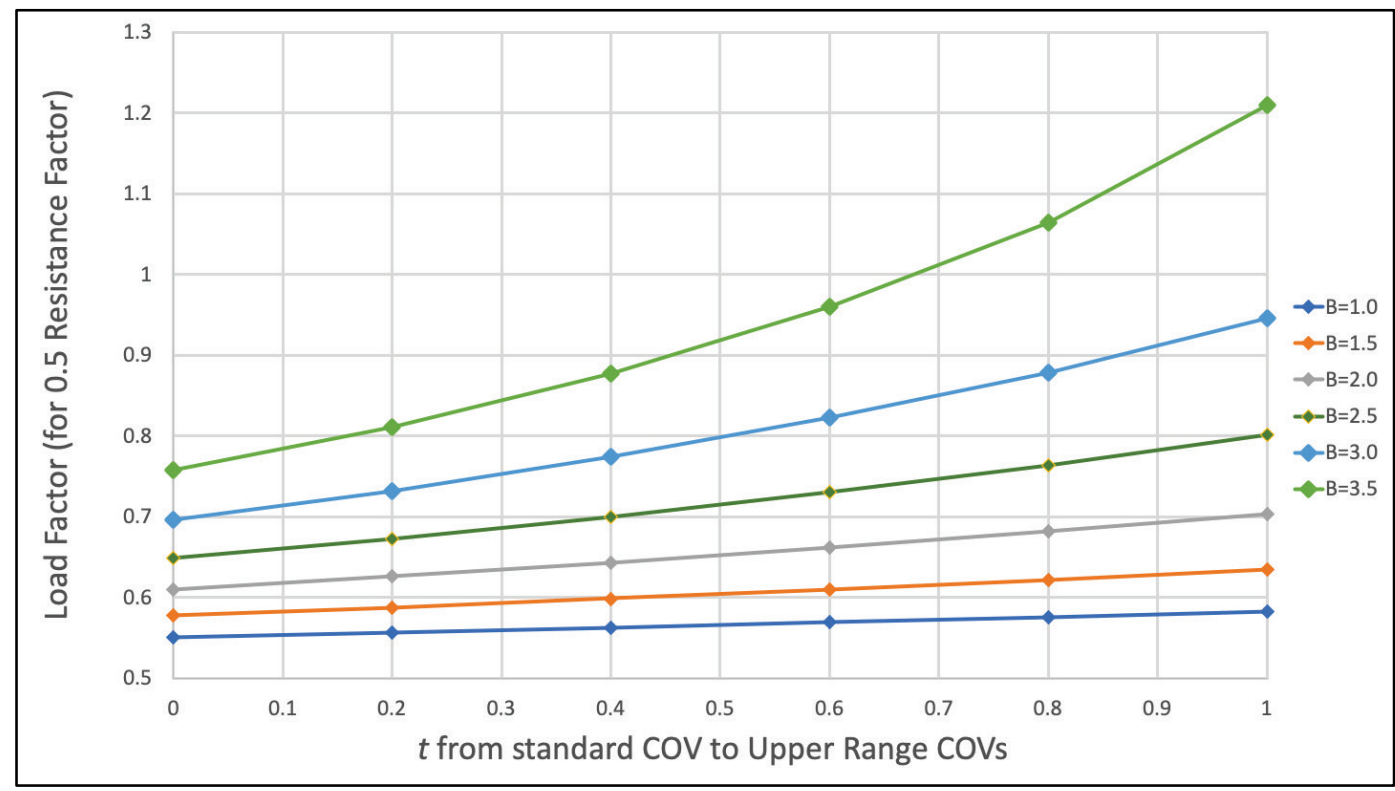

For a single load case, it is possible to map how the load factor changes as a function of $\beta$ Target. Figure 4-9 shows how load factors increase as $\beta$ Target increases for the system with Upper Range COVs for the Sliding Limit State. The relationship resulting from the simulations is non-linear due to the probability densities decreasing at distances further from the mean. The curve is smooth for $\beta \mathrm{s}$ from 1 to 3.5 because there is an adequate number of samples in those regions to make fairly accurate predictions. The curve begins to show some irregularity in results for $\beta$ values of 3.5 to 4 because the number of simulation samples in that region, due to low probability density, are likely smaller than required. However, the authors feel that the trend is adequately captured in these results and no further simulation analyses with larger sample sizes are warranted. Computing load factors for higher $\beta$ values would require performing a much greater number of simulations. The form of the curve is apparent in Figure 4-9, even with the recognized instabilities in the tail of the distribution. 
Figure 4-9. Upper Range COV value based computed Sliding Limit State Load Factors for increasing $\beta_{\text {Target, }}$ all for a Resistance Factor of 0.5 .

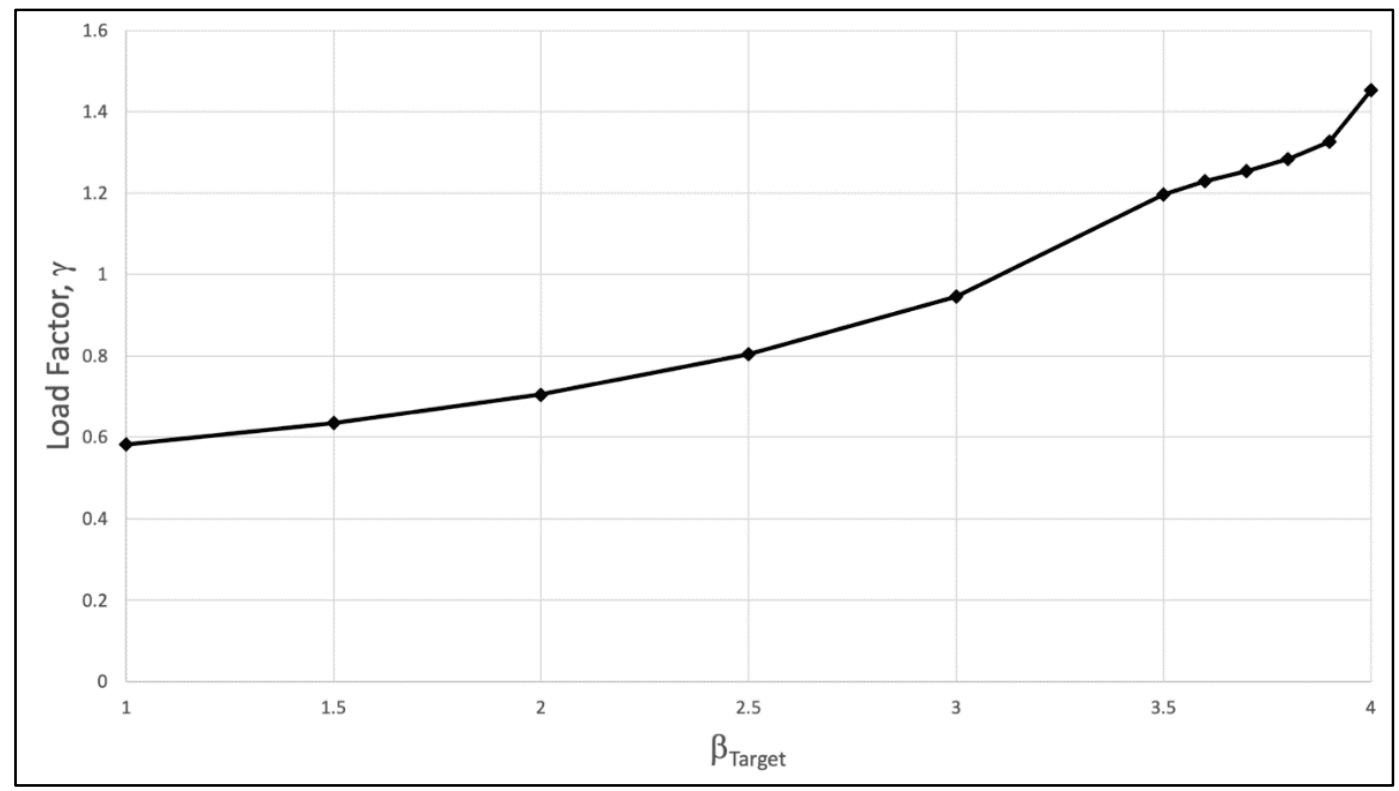

\subsection{Conclusions}

\subsubsection{Results from Original, Mid-Range COV values for effective angles of friction}

Section 4.1 presents an assessment of existing limit state computations with respect to geotechnical problems, specifically the example problem described in Chapter 2. The traditional Reliability-based methods rely on defined (e.g., Gaussian) and uncorrelated distributions with continuity of slope for load and resistance (e.g., Nowak and Collins 2013). The Example 2 geotechnical problem creates load and resistance distributions that are not well-defined, continuous, and non-correlated because the loads and resistances are computed from normal distribution soil properties with conditional equations where all of the soil properties are used for load and resistance.

Section 4.2 introduces another procedure for determining load scale factors for individual limit states that relies on direct Monte Carlo simulation given values recovered from distributions of the material properties (i.e., $\phi_{1}^{\prime}, \phi_{2}$, and $\phi_{3}^{\prime}$ ). Recall that these three shear strength parameters are defined as independent, normally distributed variables in the Chapter 2 T-Wall problem. From these simulation runs, correlated resistance and load samples are computed in the units consistent with each limit state. A scale factor for the existing resistance samples can be searched for numerically to find the value such that the scaled values compared to the corresponding 
load samples yields the probability of unsatisfactory performance desired. This resulting Resistance Scale Factor is then converted to a Load Scale Factor in the devised analytical procedure. Given the correlation, and changes of load and resistance values as the design changes, this process may need to be iterated to achieve the desired accuracy for the desired PUP.

Using the Example 2 design of a T-wall with a base of $25 \mathrm{ft}$ under the Usual load condition and Extreme load condition, each of the three geotechnical limit states were assessed for the Load Scale Factor determined by the Section 4.2 procedure. The biggest indicator that a limit state is more likely to occur than another is the Resistance Scale Factor. The greater the value of $R_{S F}$, and especially if it exceeds 1.o, indicates the greater PUP values. Because the range between load and resistance distribution means can vary by large amounts between limit states, the $L_{S F}$ value does not reveal the PUP status between limit states.

In traditional steel structure LRFD design, the variation in both the nominal load and resistance can be estimated with more certainty than the resistance and load in a Geotechnical limit state involving soil structure interaction. Soil strength properties are measured sparsely and are spatially correlated. Generally speaking, the dispersion in Geotechnical strength properties are greater than for structural steel and concrete strength properties. In addition, the dispersion in Geotechnical strength properties contribute to the dispersion of the resisting and load forces applied to the structural wedge by the driving wedge and by the resisting wedge of the three wedge system formulation that are used in the three Geotechnical limit state evaluations of Sliding, Base Area in Compression, and Bearing.

Note that using USACE criteria for allowable stress design (EM 1110-22100) (HQUSACE 2005) resulted in a base width for the example T-Wall being $25 \mathrm{ft}$. Under Normal Load conditions, the PUP initial for this structure is 0.00025 , and the resulting $\beta$ initial is over 3.5. However, LRFD is about improving reliability under Extreme load conditions, which by definition, happen infrequently. According to Figure 4-5, extending the base width of the wall to $26 \mathrm{ft}$ will provide satisfactory performance corresponding to a value of $\beta$ greater than 3.0. To approach a $\beta$ target of 3.5, the computations show that the base width would have to be extended to $32 \mathrm{ft}$.

However, extending the base width to $32 \mathrm{ft}$ may be unnecessary, at least according to traditional USACE guidance. The Base width design is based 
on FS according to design using the CTWALL (Pace 1994) software. This design meets the standards of EM 1110-2-2502 (HQUSACE 1989) and, therefore, the EM 1110-2-2100 (HQUSACE 2005) guidance for the FS for sliding of a structure under Normal loading using the Ordinary Site Information Category. This FS under ASD of 1.5 yields a design $\beta$ of 3.48 under the Normal Load case, using Monte Carlo simulations.

Using the EM 1110-2-2100 (HQUSACE 2005) guidance for the FS for sliding of a structure under Extreme loading using the Ordinary Site Information Category, the $F S$ should have a minimum value of 1.1. The lowered safety factor reflects the fact that Extreme loading is an infrequent and short duration occurrence, so the PUP value is affected by how often these events occur as well as their duration. CTWALL software executions with the Extreme Load case yielded a $F S$ of 1.43, which exceeds this ASD guidance limit of EM 1110-2-2100 (HQUSACE 2005). Monte Carlo simulations give this limit state a yield $\beta$ of 2.92. This value affirms the selection of lower $\beta$ values in the work of Fenton et al. (2016), where a $\beta$ of 2.5 was used for soil-structure interaction for bridges but also reveals that the new proportioned load procedure allows for greater accuracy in $\beta_{\text {target }}$ calculation over the Fenton approximations. The authors suggest that $\beta$ target values for the SSI limit states can approach values of 3.0, resulting in safer PUP values.

This leads the authors to conclude that for this example problem in which the sliding limit state controls the T-Wall geometry, a $\beta$ target for the Extreme load case can be defined to be less than the $\beta$ target for the normal load case. Using the proportioned load procedure with an appropriate limit state model and correctly conditioned Extreme Load cases, greater accuracy can be obtained resulting in lower PUP values.

\subsubsection{Results from Upper Range COV values for effective angles of friction}

LRFD results are based on Extreme events, where limit state boundaries are exceeded. To achieve those conditions, the most severe conditions that the designed T-Wall will be put under were used (Figure 4-3 b). However, to adequately understand the variability in the model, Upper Range COV values for known distributions of the independent variables $\phi^{\prime} D W, \phi^{\prime} S W$, and $\phi^{\prime}{ }_{R W}$ should be used. The greater variability leads to higher Load Factors. 
In Table 6.3 of his unpublished 2015 letter report to the USACE on the topic of Procedures for Developing Reliability-Based Load Criteria for Hydraulic Steel and Concrete Structures, Professor Bruce Ellingwood recommended 100-year service life target $\beta$ values of 3.5 and 4.0 for noncritical and critical structures, respectively. This recommendation of reliability targets for LRFD is for hydraulic structures possessing a single load path.

In the Barker et al. (1991) LRFD study, resistance factors were developed using statistical data gathered from case studies obtained from published literature. Small scale-model tests provide the primary source of data for the Reliability assessment of footings in their study. The Load and Resistance factors were calibrated by fitting to ASD for different foundation systems. Allen (2005) observed that calibration by fitting to ASD influenced and frequently controlled the final value selected for the resistance factor in the Barker R\&D team's study.

Allen (2005) observes that resistance factors in general for (US) bridge and other structural designs have been derived to produce a $\beta$ value of 3.5 , corresponding to an approximate probability of failure of $1 / 5,000$. In Allen's summary and interpretation of the Barker et al. (1991) R\&D team's landmark study of the reliability of different types of foundations, Barker et al. (1991) concluded that a target reliability index value of 3.5 should be used for footings and other non-redundant systems. This recommendation is not universal to all foundation types. For example, separate target $\beta$ value recommendations were made by Allen and other engineers that he cited for individual and group pile foundations. Driven piles were distinguished from bored piles in these target $\beta$-value recommendations.

Fenton et al. (2016) observe that according to the probabilistic based Canadian Bridge Design Code (CSA 2014), a typical geotechnical system might have a target maximum lifetime failure probability, $p_{f}$, of 1/5,000 $\left(2.0 \times 10^{-4}\right)$ for an Ultimate Limit State with a typical consequence level. Canadian bridges are designed for a 75-year design life. A value for $p_{f}$ of 1/5,000 for a 75-year design life structure has to be adjusted to account for the fact that USACE hydraulic structures have a design life of 100 years. This adjusted value, given the longer structural design life, is reported in Table 2-8 of Ebeling and White (2019) and corresponds to a PUP value of $1 / 3,475\left(2.67 \times 10^{-4}\right)$. This PUP value is equivalent to a $\beta$ value of 3.46 . Again, according to Fenton et al. (2016), the Canadian Bridge Design Code 
(CSA 2014) provides guidance for a target maximum lifetime failure probability, $p f$, of 1/10,000 for an Ultimate Limit State with a high consequence level. Ebeling and White (2019) in their Table 2-8 report for a 100-year design life, a corresponding PUP value of 1/7,519 $\left(1.33 \times 10^{-4}\right)$ and its equivalent $\beta$ value of 3.65. Summarizing, the probabilistic Canadian Bridge Design Code data would infer a range in $\beta$ between 3.46 and 3.65 for typical to high consequence levels in a 100-year design life structure.

Table 3-1 in EM 1110-2-2104 (HQUSACE 2016) guidance for the Extreme load case for lateral earth pressures provides values for a Resistance Factor of 0.5 and a Load Factor of 1.4. A Load Factor value of 1.4 corresponds to a Reliability Index value of approximately 3.8 by the data given in Figure 4-9 for Upper Range COV values for effective angles of internal friction. The $\beta$ value of 3.8 for a USACE Navigation Structure falls approximately midway between the Ellingwood reliability index recommendation for normal and critical structures. This computed $\beta$ value of 3.8 exceeds the historical ASD foundation design $\beta$ experience (Allen 2005) as well as the guidance for the Canadian Bridge Design Code as described by Fenton et al. (2016). Thus, the EM 1110-2-2104 (HQUSACE 2016) guidance LRFD values for load and resistance factors for the driving and resisting earth pressure loads applied to the Structural Wedge in the sliding limit state are deemed slightly conservative but not excessive by the authors of this report when a $\beta$ of 3.8 value for the dominant limit state of sliding is compared to the target $\beta$ values given by Allen (2005) and Fenton et al. (2016). This assessment may be improved by making a Reliability assessment of other earth-retaining USACE hydraulic structures following the procedure of analysis outlined in this technical report.

Using Figure 4-9, a load factor of 1.2 is sufficient to achieve a target $\beta$ equal to 3.5 for the dominant limit state of sliding, which is required according to Ellingwood for a noncritical structure with a single load path, given a specified resistance factor of 0.5 according EM 1110-2-2104 (HQUSACE 2016) guidance. This Load Factor value is $14 \%$ lower than the reported EM 1110-2-2104 (HQUSACE 2016) load factor of 1.4 with the same specified resistance factor. 


\section{A Numerical Method for Computing Multiple Limit States Probability of Unsatisfactory Performance (PUP) and Load Factors for the Example Soil- Structure Interaction of the T-Wall Model with Material Variability}

\subsection{Combining multiple limit states}

Chapter 3 introduced procedures for calculating the $g(\boldsymbol{X})$ functions for each of the individual limit states that can occur for the T-Wall example problem specified in Chapter 2. Each $g(\boldsymbol{X})$ function is created based on the computed loads and resistances, with failure happening when the load exceeds the resistance, a natural conclusion of Equation 2.52. The loads and resistances are computed from the variable soil properties of vector $\boldsymbol{X}$ and expected water levels in the geotechnical three wedge solution method, using Driving, Resisting, and Structural Wedges. Chapter 2 concluded by stating that lower values of $g(\boldsymbol{X})$ are not desired, with values less than the response surface (where $g(\boldsymbol{X})=0.0$ ) indicating failure or unsatisfactory performance.

To compute the combined PUP for all of the limit states for the T-Wall example problem, there needs to be an overarching performance function that accounts for all the limit states at once. Because lower values are not desired, this performance function can be computed as

$$
g_{M L S}(\boldsymbol{X})=\min \left(g_{1}(\boldsymbol{X}), g_{2}(\boldsymbol{X}), g_{3}(\boldsymbol{X}), g_{4}(\boldsymbol{X}), g_{5}(\boldsymbol{X}), g_{6}(\boldsymbol{X})\right)
$$

where

$$
\begin{aligned}
g_{M L S}(\boldsymbol{X}) & =\text { the Multiple Limit State performance function } \\
g_{1}(\boldsymbol{X}) & =\text { the overturning limit state } \\
g_{2}(\boldsymbol{X}) & =\text { the sliding limit state } \\
g_{3}(\boldsymbol{X}) & =\text { the bearing limit state } \\
g_{4}(\boldsymbol{X})= & \text { the stem shear limit state } \\
g_{5}(\boldsymbol{X})= & \text { the stem moment limit state, } \\
g_{6}(\boldsymbol{X})= & \text { any additional limit state (e.g., based upon limiting } \\
& \text { deformation for the T-Wall). }
\end{aligned}
$$


For PUP calculations, the exact value of a performance function is not necessary. It is only necessary to report if the performance function exceeds o.o or not, which determines if the performance is satisfactory or not. This leads to a response surface indicator function, given as

$$
i_{M L S}(\boldsymbol{X})=\left\{\begin{array}{l}
0: g_{M L S}(\boldsymbol{X}) \geq 0.0 \\
1: g_{M L S}(\boldsymbol{X})<0.0
\end{array}\right.
$$

For this multiple limit state indicator function a value of o indicates satisfactory performance while a value of 1 indicates unsatisfactory performance.

Figure 5-1 shows a probability-based two-variable space with multiple limit states. The two variables $\mathrm{X}_{1}{ }_{1}$ and $\mathrm{X}_{2}{ }_{2}$ shown in this figure are presented in the transformed basic variable space, as described in Section 2.4 of Ebeling and White (2019). The concentric, blue circles shown in this figure represent the radially symmetric, normalized, Normal-Gaussian PDF distribution. Observe that the PDF is isotropic in this transformed, 2-D space. The Reliability Index $\beta$ shown in this figure represents the number of standard deviations from the means of $X_{1}{ }_{1}$ and $X^{\prime}{ }_{2}$ variables of this figure's origin to the Design Point. The Design Point is the closest distance from either limit state response surface to the mean center. The response surfaces for the two limit states are identified in this figure. The area beyond the red combined response surface line (where the areas are shaded) is the region where the indicator function would return 1 , with the unshaded regions returning 0. 
Figure 5-1. A 2-D example of multiple limit states with closest approach of length $|\beta|$ in the transformed basic variable space.

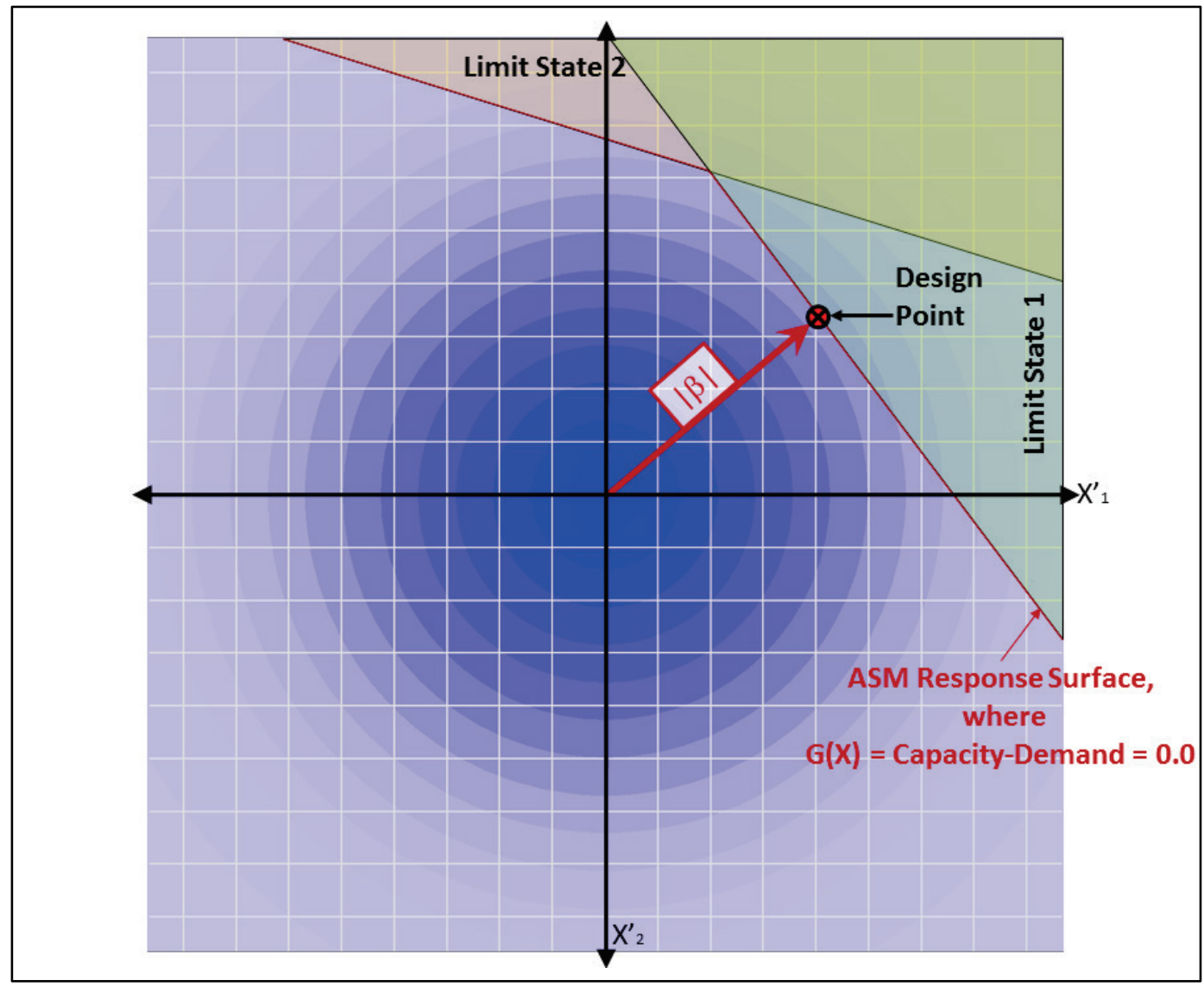

\subsection{The multivariate space for the response function}

For the Chapter 2 Example T-Wall problem, the variable vector is described as

$$
\boldsymbol{X}=\left\{\phi_{\text {Driving }}^{\prime}, \delta_{\text {Driving }}^{\prime}, \phi_{\text {Structural }}^{\prime}, \delta_{\text {Structural }}^{\prime}, \phi_{\text {Resisting }}^{\prime}, \delta_{\text {Resisting }}^{\prime}\right\}(5.3)
$$

Where the values are drawn from the distributions determined from the mean values $(\mu$ ) given in Table 2-1 and using the spatial COVs calculated according to the procedures of Chapter 3 to obtain the needed value of standard deviation ( $\sigma=\mathrm{COV}$ times $\mu$ ). Recall from Chapter 2 that the soil strength parameters $\phi$ ' and $\delta$ for all three soil regions are assumed to be normally distributed with a lower bound at $\mu$ minus $5^{*} \sigma$ and an upper bound at $\mu$ plus $5^{*} \sigma$. For simplicity in the example problem, these respective distributions are assumed to have no correlation and are drawn from Gaussian distribution. Because the distributions are not correlated, they can form orthogonal axes of a probability distribution space. Using the 
means as the origin and normalizing each distribution by its standard deviation, the variable distributions form a normalized Gaussian distribution space.

The vector $\boldsymbol{X}$ describes a point in the space, where the probability of that point is

$$
h_{M L S}(\boldsymbol{X})=\prod_{n} h\left(X_{n}\right)
$$

where $h()$ is the probability density function for the normal Gaussian distribution and $X_{n}$ is the $n^{\text {th }}$ variable of vector $\boldsymbol{X}$.

The normalized Gaussian distribution space has several simplifying properties. For instance, given a hypersphere with radius $\beta$ from the mean origin in the normalized Gaussian distribution space, every point on the surface of the hypersphere has the same probability. Another useful property is that given the same hypersphere of radius $\beta$, integrating the area of the half-space behind any hyperplane tangent to the hypersphere has the same probability as the area behind a point at that distance for an individual normal distribution.

\subsection{Calculation methods for PUP}

\subsubsection{Advanced second moment (ASM) method for a single limit state}

The ASM family of procedures can find the closest point on a response surface (where $g(\boldsymbol{X})=0.0$ ) from the origin at the mean values for the normalized Gaussian distribution space. This closest point is called the design point (Figure 5-2). The vector from the mean origin to the design point has distance $\beta$ and can be normalized to a unit vector that has the directional cosine values for each of the axes, $\alpha$. This vector is perpendicular to the response surface if the response surface is hyperplanar. From the description of the normalized Gaussian distribution space, the PUP value of this single limit state is the same as one minus the single normal distribution CDF value for $\beta, 1-\Phi(\beta)$ (Figure 5.3).

The ASM methods use the same procedure to find the design point. Using the derivative to find the slope toward the response surface, the ASM method walks along the surface to find the local minima distance between the mean origin and the response surface. 
Although it is not a method that can be used for multiple limit states, the ASM methods can serve a useful purpose for proposed methods to calculate $P U P$. For instance, bound-finding methods combine multiple hyperplanar equations to determine the effective range for PUP values. Another example is the use of the design point for Importance Sampling, which will be discussed at the end of Subsection 5.3.3.

Figure 5-2. A 2-D example of the PUP region and the hyperplane description using $\alpha$ and $\beta$ determined using ASM methods.

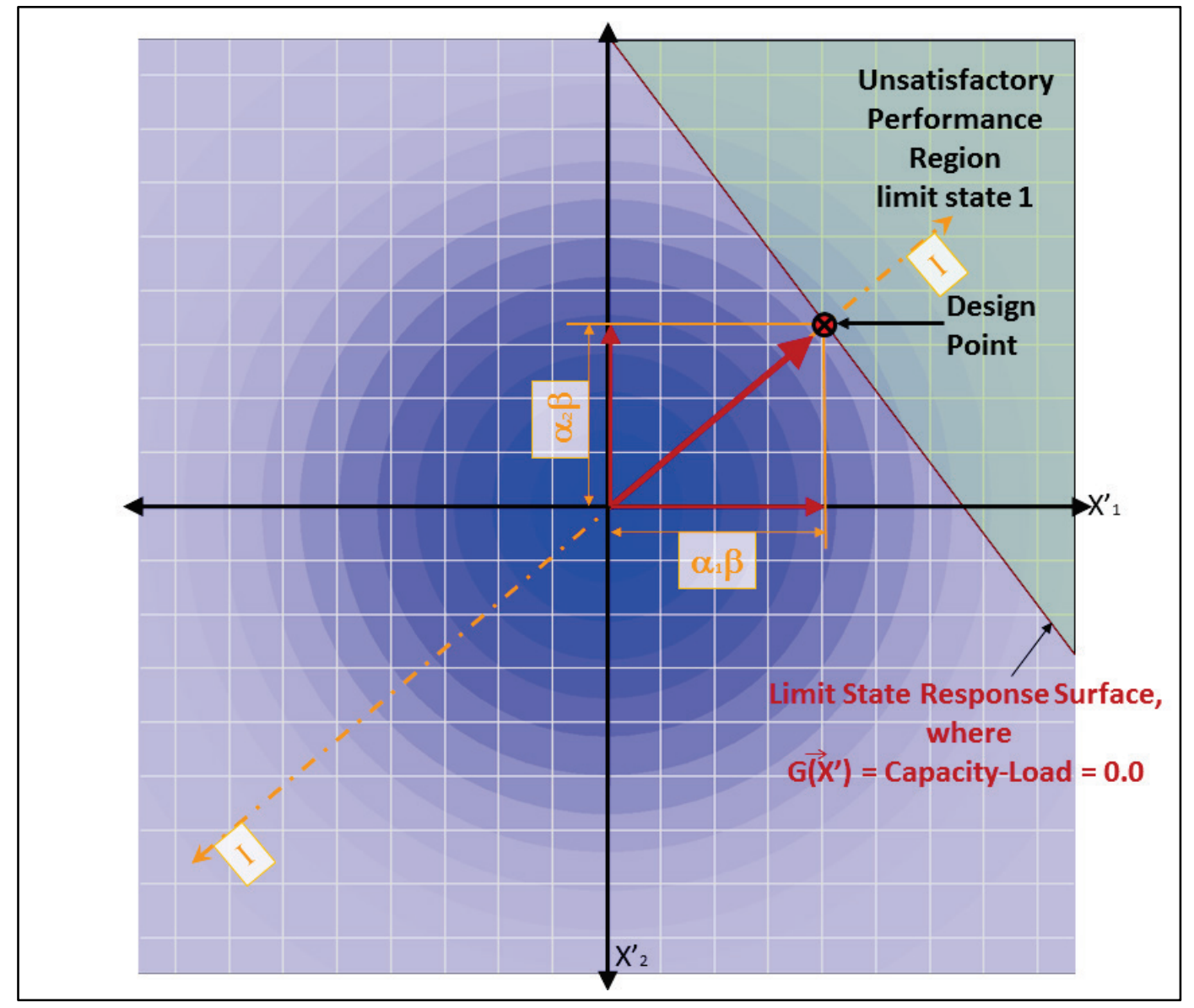


Figure 5-3. Transferring the hyperplanar limit state to the single normal axis.

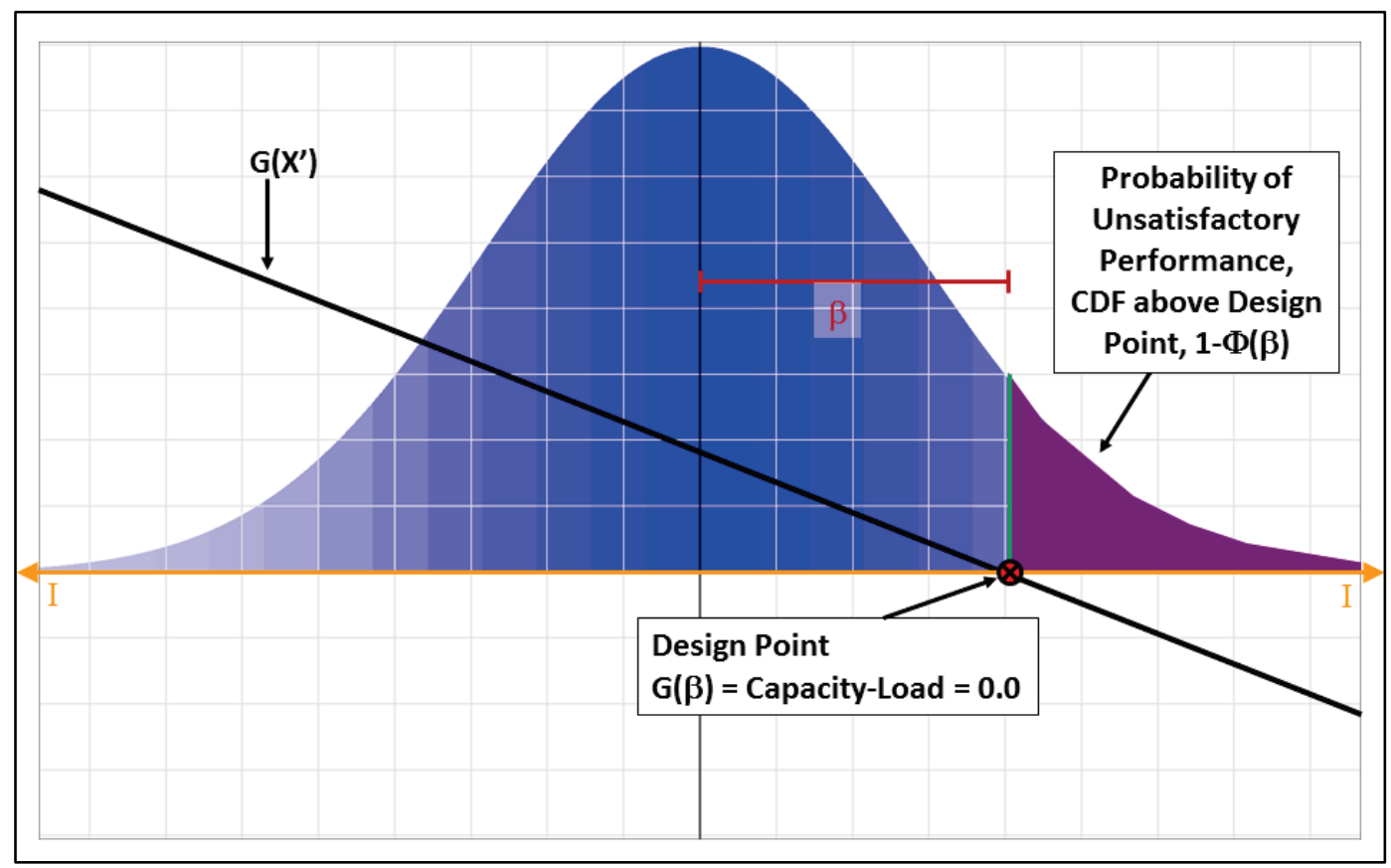

\subsubsection{Numerical solutions for PUP}

\subsubsection{Direct Euler numerical integration}

Given a normalized Gaussian distribution space, the PUP value can be computed by integrating the space with the indicator function. Equation 5.5 gives an integration for PUP using Euclidean coordinates in the normalized Gaussian distribution space. Figure 5-4 shows how this integration is performed numerically by discretizing the probability space of 2-D vector $\boldsymbol{X}$.

$$
P U P_{M L S}=\Phi(\boldsymbol{X})=\left\{\begin{array}{c}
\int_{X_{1}} \int_{X_{2}} \ldots \int_{X_{N}} i_{M L S}\left(X_{1}, X_{2}, \ldots, X_{N}\right) * \\
h_{M L S}\left(X_{1}, X_{2}, \ldots, X_{N}\right) d X_{1} d X_{2} \ldots d X_{N}
\end{array}\right\}
$$

To find an approximate design point (the location of unsatisfactory performance that is closest to the mean origin), the center location of the grid point needs to be determined. A best distance measure is initialized to infinity for the start. As the integration proceeds, if unsatisfactory performance has been found, then the distance from the gridpoint center to the mean origin is computed. If this distance is less than the previous best distance, then the new design point is set to the gridpoint center and the best distance is set to its distance to the mean origin. This design point has an error no bigger than the distance from corner to corner of a grid cell. 
Figure 5-4. Numerical integration and computation of a PUPvalue with two variables with Gaussian distributions given a single hyperplanar limit state (using Euclidean coordinates).

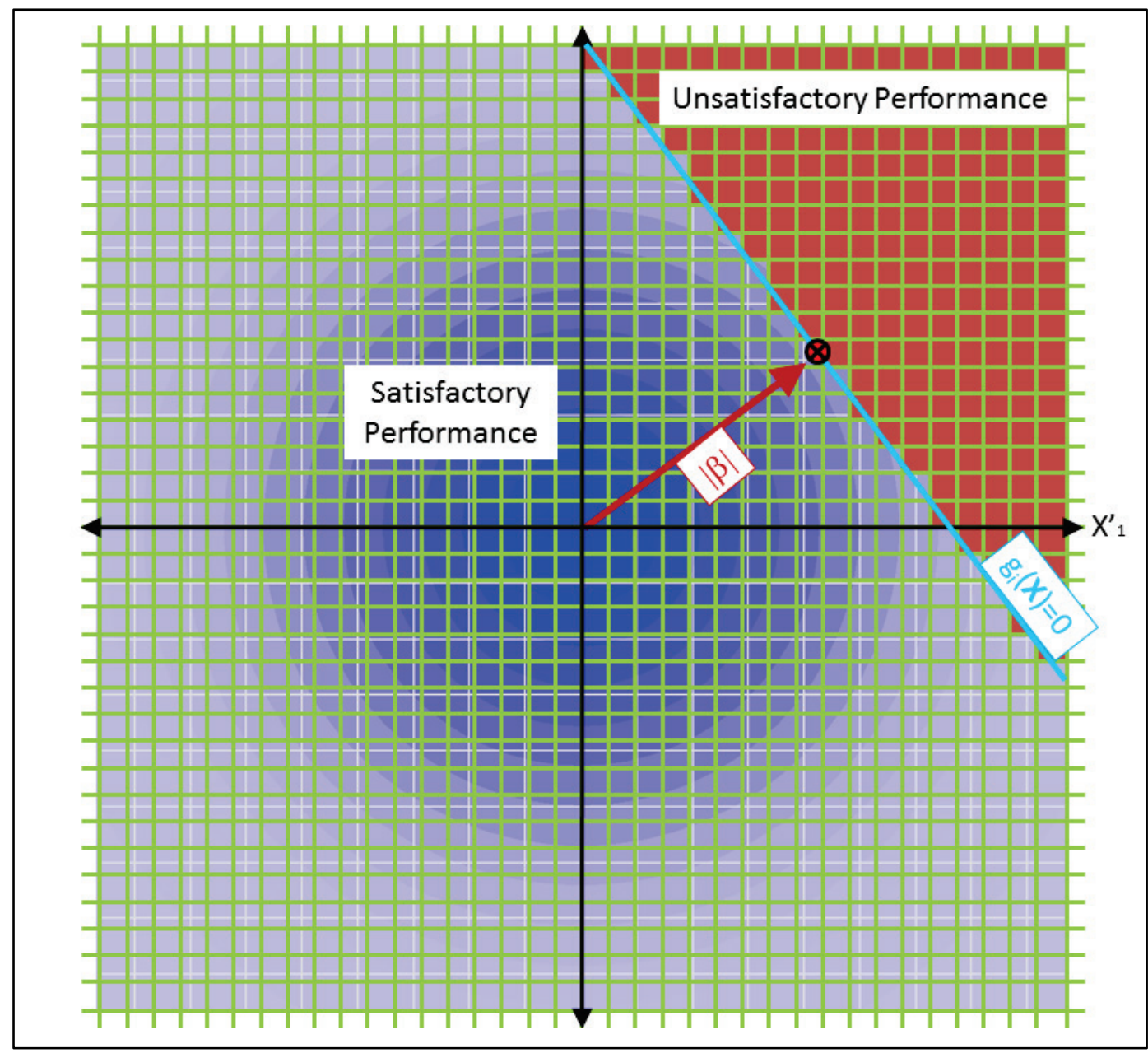

\subsubsection{Adaptive hyperspherical Gaussian probability numerical integration}

Another numerical method to integrate this area has properties that can optimize algorithmic run times. This method is to use polar coordinates instead of Euclidean coordinates. Because the probability at a certain radius has the same probability for any angles, varying the radius first reduces the number of times a probability has to be calculated for the hyperspherical shells. The integration in polar coordinates is given as

$$
\begin{aligned}
& P U P_{M L S}=\Phi\left(r, \varphi, \theta_{1 \ldots N}\right)= \\
& \int_{r} \int_{\varphi} \int_{\theta_{1}} \ldots \int_{\theta_{N}}\left\{\begin{array}{c}
i_{M L S}\left(r, \varphi, \theta_{1}, \theta_{2}, \ldots, \theta_{N}\right) h(r) r^{N} * \\
\sin ^{N-1}\left(\phi_{N}\right) \sin ^{N-2}\left(\phi_{N-1}\right) \ldots \sin \left(\phi_{1}\right) d \phi_{N} d \phi_{N-1} \ldots d \phi_{1} d \varphi d r
\end{array}\right\}
\end{aligned}
$$


where the polar-coordinate version of function $i_{M L S}()$ is computed by converting the coordinates to Euclidean coordinates and solving in the previous fashion.

To find an approximate design point (the location of unsatisfactory performance that is closest to the mean origin), it is only necessary to store the gridpoint centroid of the first unsatisfactory result. Since the radius proceeds outward from the mean origin, this point is at the shortest distance to the limit state response surface. Because of perspective effects, the error in this method increases with the distance from the mean origin.

\subsubsection{Hybrid adaptive hyperspherical Gaussian probability numerical integration using ASM}

Because of the number of dimensions and the necessity of high-accuracy solutions, hyperspherical probability integration methods suffer from exponential growth, which leads to rapid growth until the solution computation becomes intractable. Methods that restrict the volume to be integrated and the step size for computational summation can help mitigate this growth restraint, as well as restricting the number of variable dimensions. This section provides methods to restrict the volume to be searched and the step size as the integration is performed.

Previously, there have already been provided limits on the volume to be searched by limiting the search to $\beta$ radius values less than 5.0 standard deviations. This limitation is based on the normalized Gaussian distribution space, which has a cumulative probability that is less than 10e- 6 beyond the 5.5th standard deviation, or in the tails of the distribution. This restriction can be further limited by recognizing that each limit state is defined by a hyperplane that can be defined with $\beta$ radius and $\alpha$ vector using the ASM methods. Because the integration solves for the PUP, the range of the solution only needs to progress from the $\beta$ value for the limit state hyperplane nearest the mean origin of the normalized Gaussian distribution space to the maximum $\beta$ value of 5.5 .

While this method can make a tremendous impact on the time to perform the probabilistic integration, this technical report uses non-hybrid method unless otherwise specified. This method is used to maintain the same numerical computations throughout to prove the utility of the complete method. 


\subsubsection{Computing PUP using simulation}

Traditional Reliability solutions rely on simulation to calculate PUP. Simulation methods work by generating $\boldsymbol{X}$ through probabilistic sampling of the constituent distributions. Because samples are chosen according to the distribution, a large sample set begins to approximate the probability density of the area of the space. Thus, for $n$ samples, the PUP value can be calculated as

$$
P U P_{M L S}=\frac{\sum_{n} i_{M L S}\left(\boldsymbol{X}_{n}\right)}{n}
$$

where $X_{n}$ is the nth sampled point.

For a typical Monte Carlo simulation, samples are chosen completely at random. Because so many samples are chosen in high-probability regions of the space, it takes a large number of points to accurately map the normalized Gaussian distribution space.

Another sampling method called Latin Hypercubes divides each probability axis into a fixed number $n_{L H}$ of equal probability regions. To create a set of samples, each axis is assigned one of its probabilistic regions at random. Once selected, that probabilistic region cannot be selected again for that axis. A sample point is selected within the selected hyperregion. This is done until $n_{L H}$ samples have been selected. At this point, the process to calculate Equation 5.7 is performed. Because the normalized Gaussian distribution space is divided according to the distributions and points are guaranteed to be selected from every region for every variable, fewer samples are necessary for Latin Hypercubes than for the simple Monte Carlo technique.

Both Latin Hypercube and Monte Carlo simulation suffer issues with accuracy when the area of interest (in this case, the response surface and unsatisfactory region) has very low probabilities. Importance Sampling is the method to deal with this. In Importance Sampling, a point is chosen on or near a transition region and then points are chosen about the distribution at that location. The probabilities of the sample points are then transformed back to the original distributions. Because more samples are taken about the region of interest, the accuracy of the calculation is improved. To find a point on the region of interest, an Advanced Second Moment computation is performed using the $g(\boldsymbol{X})$ function to find the 
point on the response surface nearest to the origin (the design point at the original distribution mean values). The design point establishes a normalized vector $\alpha$ and a distance to the surface $\beta$. Importance Sampling is used in the USACE CASE program CPGA-R probabilistic solution procedure for the Reliability analysis of pile groups and calculation of a PUP value. Figure 5-5 depicts the governing Equations 5.8 through 5.11. The author's experience is that while Importance Sampling improves accuracy locally, it can have problems with concave response surfaces that enclose the tail ends of the distributions.

For distribution spaces $P$ and $Q$, where the points will be sampled from $Q$ but transformed to a probability in $P$ for Importance Sampling, the normalized distance that a sampled point has from each distribution space mean is given as

$$
\begin{aligned}
& \boldsymbol{\beta}_{p}=\frac{X_{i}-\mu_{p}}{\sigma_{p}} \\
& \boldsymbol{\beta}_{q}=\frac{X_{i}-\mu_{q}}{\sigma_{q}}
\end{aligned}
$$

The weighting function based on these $\beta$ vectors is then

$$
W(\boldsymbol{X})=\frac{P\left(\boldsymbol{\beta}_{p}\right)}{Q\left(\boldsymbol{\beta}_{q}\right)}
$$

Where the functions $p()$ and $q()$ represent the PDF point probabilities for the sample point $\boldsymbol{X}_{\boldsymbol{i}}$ in the two spaces. This leads to the calculation of the point chosen from the distribution space $Q$ in terms of probability in distribution space $P$ as

$$
P_{u}=\frac{1}{N} \sum_{i=1}^{N} I(g(\boldsymbol{X})) W(\boldsymbol{X}) \text { where }\left\{\begin{array}{l}
I=1 \mid g(\boldsymbol{X})<0 \\
I=0 \mid \text { otherwise }
\end{array}\right\}
$$


Figure 5-5. Importance Sampling determines the ratio between two distributions $P$ and $Q$ to calculate the true $p$ probability for the point sampled from $q$.

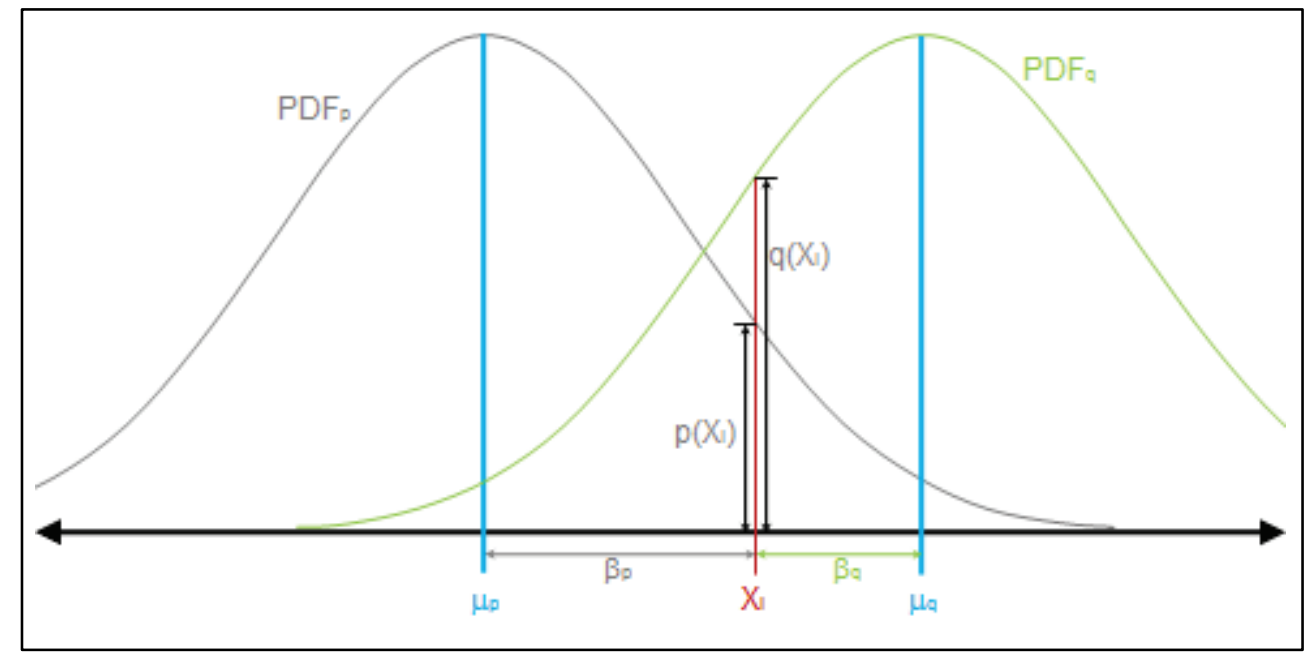

\subsubsection{Finding the limiting range (bounds) for PUP}

Ang and Tang (1984), in an effort to reduce time to compute the value of $P U P$ with multiple hyperplaner response surfaces, decided to use logical methods based on DeMorgan's rule for the combination of logical probabilities.

There are limited technical publications reporting on probabilistic investigations of multiple Geotechnical limit states and multiple failure modes. Fan et al. (2014) discuss the Reliability analysis of piles in spatially varying soils considering multiple failure modes. Specifically, they summarize their research into the performance state of vertical drilled shafts under combined lateral and axial loading using a Reliability analysis. The performance of the vertical pile is defined in terms of the displacements induced by external loading. They consider three performance limit states: lateral pile deflection, angular pile distortion, and axial pile movement at the top of shaft. The unsatisfactory performance event is said to occur if the induced displacements at the top of the pile are greater than the corresponding allowable displacements. Lateral pile response is computed using the p-y method of pile-to-soil interaction analysis, and vertical displacements are computed using a t-z model for pile-to-soil interaction. ${ }^{1}$ Uncertainties in soil deformation and strength properties are considered in their Reliability analysis. Each of the soil property variables used in the

\footnotetext{
1 Examples of the USACE CASE programs that perform lateral $p-y$ analysis and axial t-z analysis are COM624G and CAXPILE, respectively.
} 
analytical model are characterized by values of mean, standard deviation and distribution type (e.g., normal, lognormal). With the statistical properties defined, a Monte-Carlo simulation method is used to conduct the Reliability analysis. They apply a conventional Reliability methodology of computing a $P U P$ value for each of the three displacement limit states as well as a PUP value for the system.

One of the important findings of the Fan et al. (2014) study is that the PUP for the system is greater than for any of the three individual displacement limit state modes and is less than the sum of the PUP values of the three individual limit state modes if assuming the statistical independence among the three limit state modes. Fan et al. (2014) emphasize that the value for $P U P$ will be underestimated if multiple displacement limit state modes are not considered simultaneously while the PUP value for the system will be overestimated if the dependence among the different displacement limit states is not considered.

The USACE hydraulic structures are designed to resist multiple limit states, which are listed in Section 1.5. When a hydraulic structure is viewed in terms of a Reliability methodology, Fan's research indicates that the interrelationship between multiple performance limit states and a system $P U P$ value is an important consideration. This is one of the research tasks investigated by the authors of this report, with the initial findings discussed in the Phase 1 portion of the two-phase research study, in Chapter 4 and Appendix A of Ebeling and White (2019).

Accommodating multiple limit states and/or failure modes given multiple variables in the PUP calculation has been shown by the Fan et al. (2014) study to be a requirement of a Reliability analysis. Therefore, Appendix A describes different methods that have been researched and/or devised by the authors of this report to estimate the overall PUP values for systems with multiple limit state response surfaces.

For a single Limit State with a hyperplanar Limit State Response Surface, as shown in Figure 5-6, the calculation of PUP is simple and accurate. Because the variables are mapped to a normalized multivariate Gaussian distribution space, the integration of the area of unsatisfactory performance on one side of the hyperplane of distance $\beta$ (at the design point) from the mean origin is the same as the area for the single variable normalized Gaussian distribution beyond the design point distance $\beta$. 
Figure 5-6. Mapping the integrated PUPfrom the multivariate hyperplane to a single Gaussian distribution.

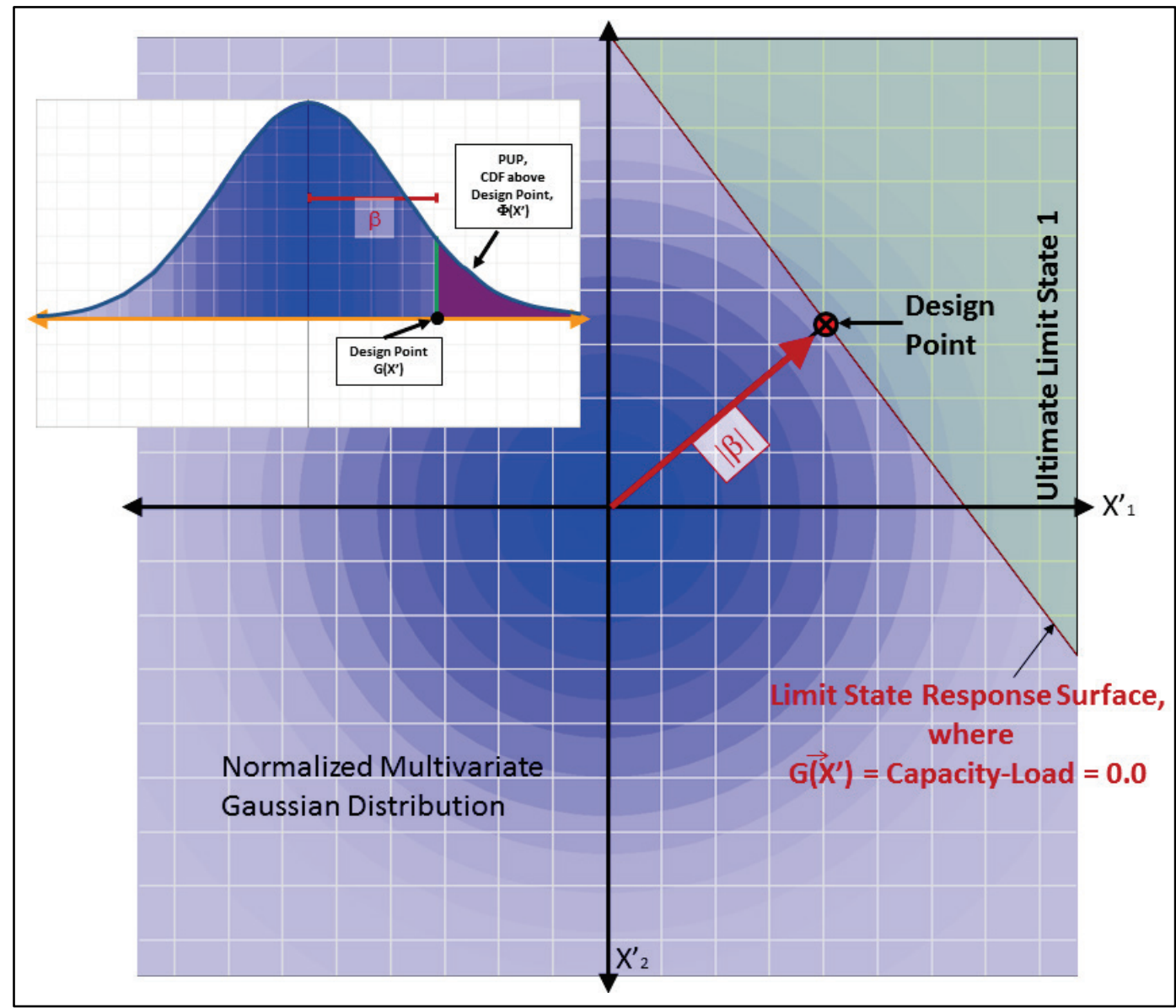

This situation becomes more complicated as non-hyperplanar limit state response surfaces are considered. Figure 5-7 shows how two hyperplanar limit states combine to create a non-hyperplanar limit state response surface. In this case, the integral of the point value PUP probabilities includes the probabilities under the second limit state that does not overlap the first limit state probabilities. This is represented by the Figure 5-7 probability distribution area that is beyond the limit state response surface where the regions are shaded red, green, and khaki. The probabilities of the overlap of the second limit state and the first limit state only need to be accounted for once. 
Figure 5-7. Mapping the integrated PUPfrom the multivariate hypersphere to a single Gaussian distribution.

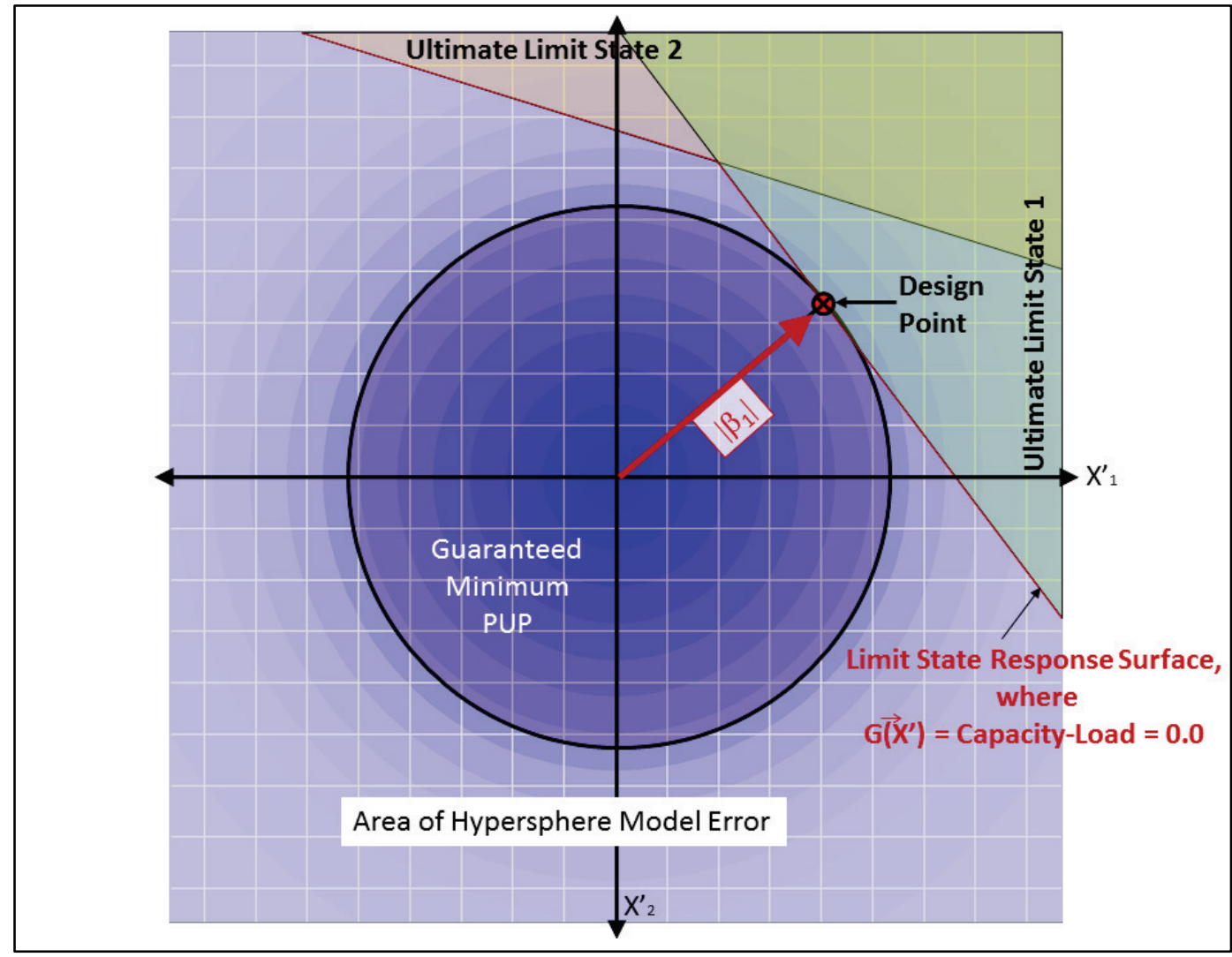

Recall from Ebeling and White (2019), the reliability of the system is defined as the complement of the probability of unsatisfactory performance (1-PUP) in their Equation 2.10. Because the ASM design point exists at the point where the limit state response surface is nearest the origin of the normalized multivariate Gaussian distribution space, a way to conservatively estimate the compliment of the PUP value so that satisfactory performance is guaranteed is to compute the probability of the hypersphere of radius $\beta$, as shown in Figure 5-7. Recall that the Figure 5-7 origin corresponds to the mean values for all variables. This estimate integrates the complement of PUP using the probabilities outside of the hypersphere to determine PUP. This value is conservative because the hypersphere is guaranteed to be completely in the area of satisfactory performance but is not guaranteed to encompass all of the satisfactory performance region.

More accurate range finding PUP estimation methods provide a minimal range for the upper and lower bounds of the PUP using logical combination constraints given the limit state response surfaces, their number, and 
possible overlap information. The minimal range refers to the fact that the bounds are at the closest distance from each other based on the logical combinations. The authors of this report suggest that by choosing a value at the midpoint of the range will guarantee an estimate that is accurate to within half the range width. Ang and Tang (1984) provide methods for finding the bounds of the overlap of regions based on Bayesian logic. These estimations are based on non-correlated variables, so they involve perpendicular response surfaces. The overlap regions involve the combination of comparing each limit state with respect to each other, as shown in the two-limit state, 2-D example of Figures 5-8 and 5-9. The upper and lower bounds for the overall PUP would be the different summations and removals of probabilities based on the Bayesian combinations of non-correlated values as described in Subsection A.3.1 of Appendix A. The difference in the bounds is described by the third overlap region between overlap regions $A$ and $B$ from -5.8 and $5-9$. Note that the bounds can be found for multi-variate, multi-limit state problems.

Figure 5-8. Finding the overlap region A given limit state I with respect to limit state J.

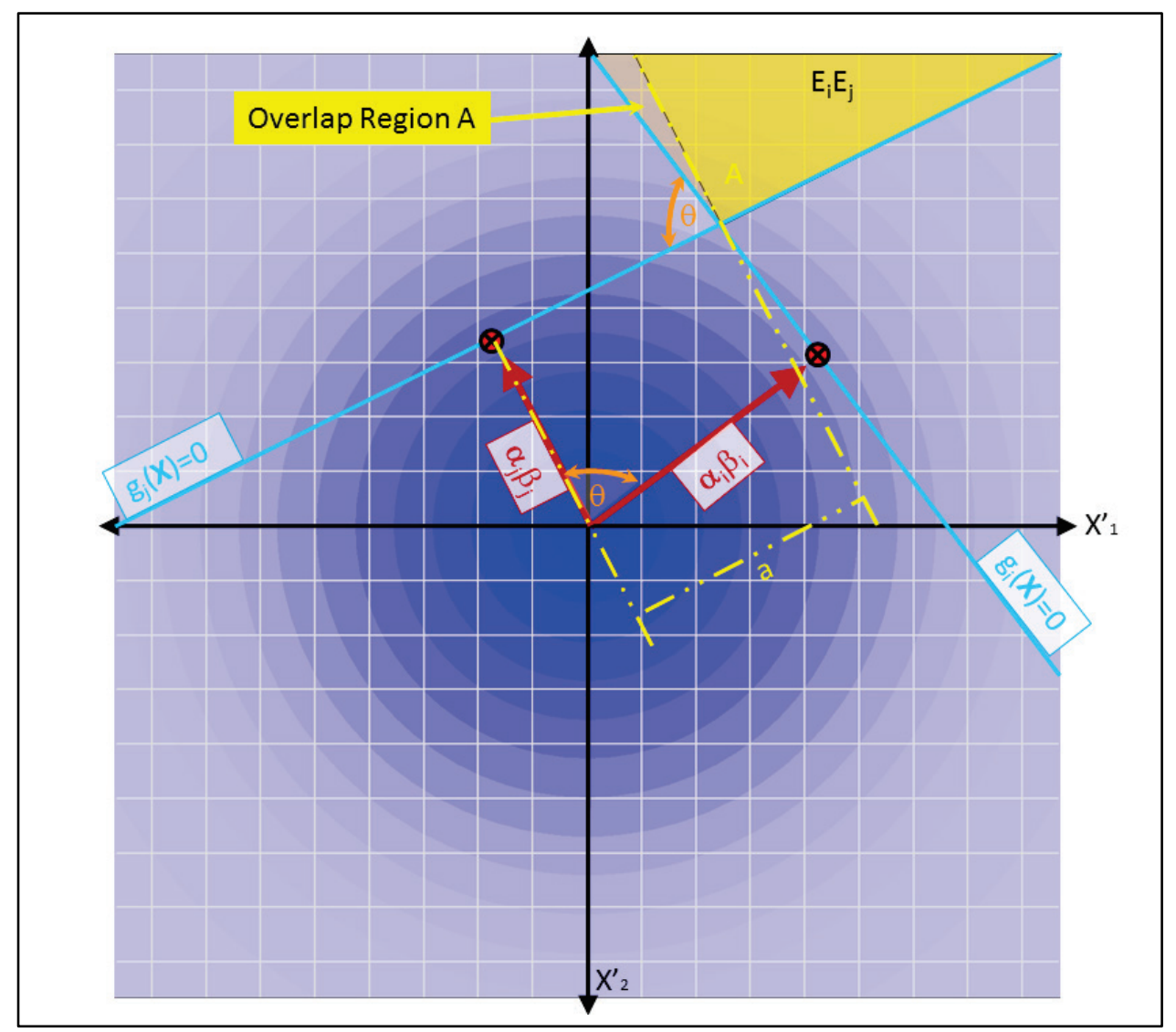


Figure 5-9. Finding the overlap region B given limit state J with respect to limit state I.

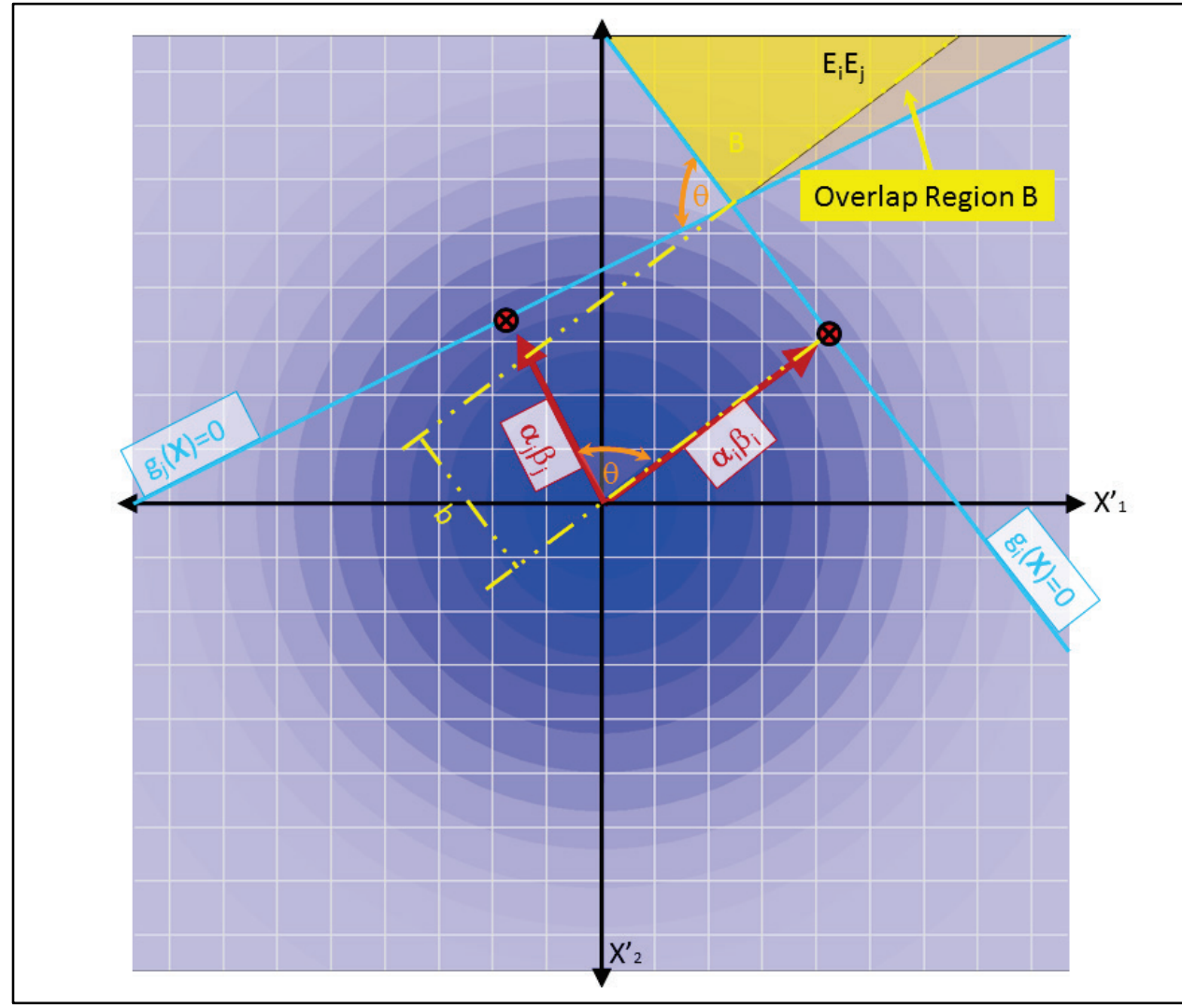

The range-finding methods were developed at a time (e.g., Ang and Tang 1984) when computational power was not widely available to perform the more accurate numerical integration techniques that are required to handle correlation between limit state response surfaces. This computational power is now available for a limited number of variables. However, rangefinding methods are still useful in that they provide valuable information that bounds the range in PUP value with minimal computational effort. Recalling the authors' suggestion made earlier in this subsection, a value assigned equal to the midpoint of the range will guarantee an estimate that is accurate to within half the range width.

Monte Carlo simulation methods can also be used to estimate PUP. Samples would be taken at random from the variable space according to each variables distribution then the unsatisfactory or satisfactory result of the sample would be computed. Many samples would be taken, and the ratio of unsatisfactory results to the overall number of samples would give the PUP estimate. For a full Monte Carlo simulation, a large number of samples $(>10,000)$ would be required. The Latin Hypercube sampling method reduces the number of samples required by guaranteeing that 
samples are not repeatedly chosen in the same regions. Also, Importance Sampling lowers the error in calculation of the probability, by sampling about the design point, which is the point of the lowest $\beta$ distance for all of the limit states (Figure 4-8), on the response surface for the combined limit states. To perform Importance Sampling, the design point, which gives the point on the closest limit state to the origin of the normalized Gaussian distribution, needs to be found. This can be done using the ASM techniques. A major concern for the simulation methods is that there are no guarantees that sample points will be generated in all of the limit state unsatisfactory performance regions, when multiple limit states exist. This concern is mitigated by the fact that the limit state regions that are not covered will typically have very low probabilities.

Numerical integration can also be used to estimate PUP. The concern is the possibility that, in some instances, it can quickly grow to be unsolvable in a timely manner, even on the most advanced computers. The authors believe that for a small number of variables, this method may provide results in a timely manner. Figure 5-4 shows an integration method for estimating the $P U P$ value. The space is divided into a hypergrid, and each grid cell probability would be added to the PUP if the variable values would lead to unsatisfactory performance. The two sources of error for this calculation would be the resolution of the hypergrid and the range of the calculations. By having the range vary from -5 to 5 standard deviations from the mean for each variable, the error can be minimized. An improvement to the integration method utilizes the fact that the probability of a normalized Gaussian distribution stays the same at a given radius; therefore, a polar hypergrid could be used so that the probability calculations would be quicker. These numerical integration methods are described in Appendix A Subsections A.3.2 and A.3.4. Because all of the variable spaces are mapped for integration and the solution looks for any unsatisfactory performance in any grid cell (voxel, or volume element), this solution method works for multi-variate, multi-limit state problems.

\subsection{Determining Load and Resistance Factor Design (LRFD) values from PUP}

Given a PUP value from one of the methods listed above, the goal is to determine an equivalent $\beta$ distance and a vector of directional cosines $\alpha$, so that load and resistance factors may be derived. Most of these methods compute PUP without respect to where the probabilities lie. Only the ASM 
method attempts to locate the point with the maximum probability of occurrence while the others can produce close estimates.

One possible method to determine $\beta$ from PUP is to calculate the probability of a hypersphere that encloses an area of probability in the normalized Gaussian distribution space that has an integrated probability of $1-P U P$. The radius of this hypersphere is then the $\beta$ distance. This is illustrated with Figure 5-10.

Figure 5-10. Setting radius $\beta$ for a hypersphere so the probability density outside the hypersphere integrates to the same value as the integration of the area region bounded by the limit state hyperplanes.

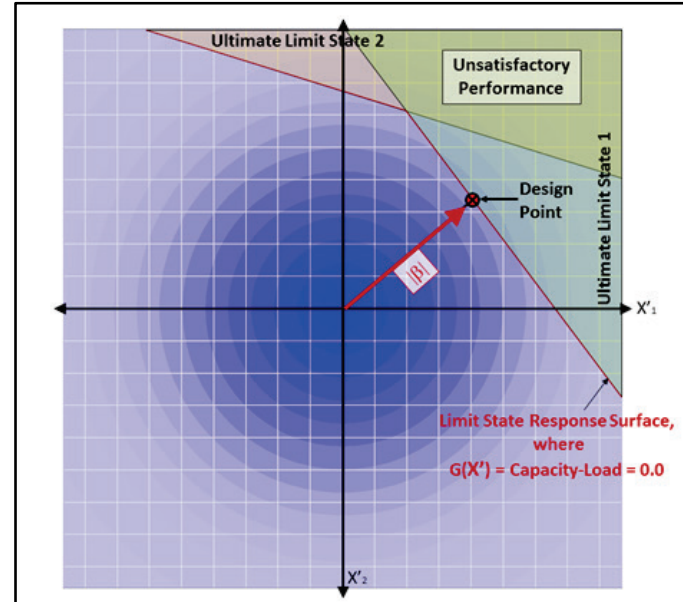

(a)

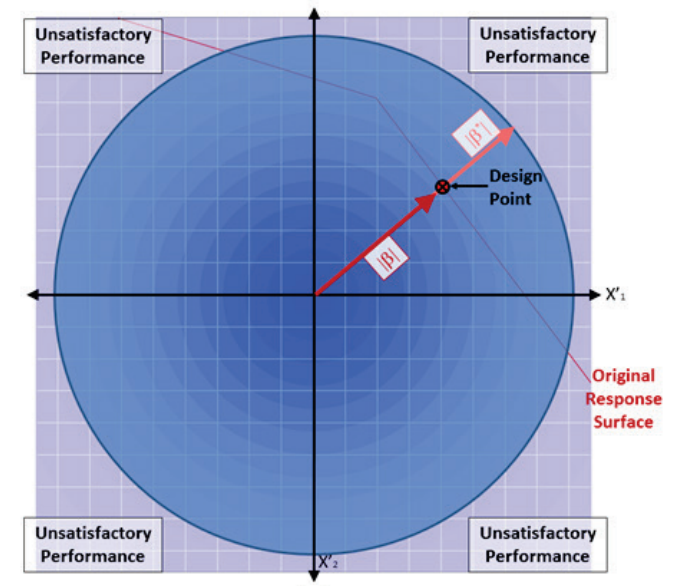

(b)

This figure shows a two-variable system where $\boldsymbol{X}=\left[X_{1}{ }_{1}, X_{2}{ }_{2}\right]$. The Unsatisfactory Performance region is defined by two hyperplanar limit states in Figure 5-10a. A design point is found using ASM. This design point is the closest point on the Response Surface (which separates satisfactory performance regions from unsatisfactory performance regions). As described previously, the unsatisfactory performance region's probability is determined accurately by integrating the point probabilities on one side of the limit state response surface (such as Equation 5.5).

For a given PUP value, Figure 5-10b shows that a hypersphere can be created that encompasses the integrated probability of the complement of PUP, which is 1.o-PUP. In this case, the Unsatisfactory Performance is the integration of the point probabilities outside the hypersphere and equivalent to the integration of probabilities to one side of the response surface in Figure 5-10a. The hypersphere has a radius of $\beta^{*}$. This can be computed without knowing the directional $\operatorname{cosines} \alpha$, which reveal the 
distance to the closest point on the response surface. Notice that $\beta^{*}$ is greater than the design point $\beta$, which means that some of the volume inside the hypersphere (which geometry calls an $n$-ball) is part of the Unsatisfactory Performance region defined by the limit state hyperplanar surfaces.

The volume of a hypersphere with dimension $n$ of radius $\beta$ is given as

$$
V_{n}(\beta)=\frac{\pi^{\frac{n}{2}}}{\Gamma_{\text {Euler }}\left(\frac{n}{2}+1\right)} \beta^{n}
$$

Where $\Gamma_{\text {Euler }}()$ is the Gamma function specified by Euler that provides a method to calculate the factorial of complex (and therefore real) numbers. An approximation of these functions can be found in many programming languages. Using Equation 5.12, the cumulative probability of a hypersphere with radius $\beta$ can be estimated by the radial integration of concentric ring probabilities. For a single concentric ring of hypersphere with dimension $n$ and radius $\beta$ and radius interval $\beta$, the probability of a point being selected in the ring is approximated as

$$
P_{\text {Ring }}(\beta)=\left(V_{n}\left(\beta+\beta_{i}\right)-V_{n}\left(\beta-\beta_{i}\right)\right)\left(P_{\text {Normal }}(\beta)\right)^{n}
$$

where $P_{\text {Normal }}()$ is the single dimension normal PDF function.

Summing these ring probabilities from the origin to any full hypersphere radius $\beta$ gives the approximate cumulative probability of the hypersphere. These data can be kept in a look-up table using small increments of the radius so that an equivalent approximate value for $\beta$ hypersphere can be computed for the probability of satisfactory performance for the response surface computed using methods that do not give $\beta$ values (i.e., simulation, integration, and bounding techniques).

System Reliability Index, $\beta$ system - Series Model: Park et al. (2015) studied the system reliability of a simple two-member truss with two failure modes for optimization purposes in design, computing the probability of failure and reliability of the truss system using a reliability simulation based computational methodology. The main goal of their paper is to discuss the reduction of computational complexity and error introduced by ignoring dependence between failure modes on evaluating system reliability, especially in the tails of the distributions, with respect to application in system optimization problems. A series reliability model, in contrast to a parallel reliability model, composed of two failure modes was 
postulated for their truss model. The series failure model is the union of failures among multiple failure modes, which implies that any single failure mechanism directly relates to system failure, as compared to the parallel failure model which requires multiple individual failures to cause system failure (i.e., an intersection of failures). A series model is judged by the authors of this technical report to be a reasonable assumption for a truss because the truss system fails if any individual truss element fails, as pointed out by Nowak and Collins (2013), in discussion of their Figure 9.4 truss. Park observes that a series model is a common failure scenario in structural design.

Structural failure of the Park et al. (2015) two-bar truss model is modeled with uncertainties for each of the two limit states being considered (i.e., tension failure in bar elements). It was assumed that the ultimate strength and the two external forces are uncertain inputs that can be described as normally distributed variables using a mean and coefficient of variation. Individual truss members are subjected to tensile force or compressive force only, depending on specific values drawn from the input variable distributions. The type of member force that developed depended upon (1) the geometric configuration of the individual trusses, (2) the strength of the individual truss members in tension and in compression, (3) the position, the orientation, (4) magnitude of the point load(s) applied to the joints in the truss system, and (5) the boundary conditions imposed on the truss system joints (e.g., none, roller, or pin). A simulation procedure, consisting of 10,000 random variable simulations, was used in their reliability computations. The system probability of failure, $P_{f \text {-system }}$, is computed using the union of failure space for the two limit states, as depicted in Figure 5 of Park et al. (2015). Their figure looks similar to Figure 5-1 of this report.

Park et al. (2015) expressed the system Reliability index, $\beta_{\text {system, as }}$

$$
\beta_{\text {system }}=-\Phi^{-1}\left(P_{f-\text { system }}\right)
$$

where $\Phi()^{-1}$ is the inverse CDF of standard normal distribution. This agrees with one of the approaches suggested earlier in this subsection and depicted in Figure 5-10 for characterizing system reliability. It was arrived at independent of Park et al. (2015). 


\subsection{Calculating PUPsystem for the Chapter 2 Geotechnical T-Wall example problem with a $25 \mathrm{ft}$ and $15 \mathrm{ft}$ base widths}

In Chapter 4, the geotechnical structural system described in Chapter 2 was evaluated for individual limit states from the soil material property distributions to determine the resistance and load distributions as well as PUP for the specified limit state. Chapter 3 revealed in Tables 3-2, 3-5 and 3.7 that the computation of spatial COVs for the soil material values used different procedures, given each of the three respective Geotechnical limit state assumptions. This means that each procedure could conceivably create very different spatial COVs for each of the three material property distributions in the limit state set. The spread of the values is determined by the original, Mid-Range point estimate standard deviation value multiplied by variance reduction factor, which was created from the slip plane length and scale of fluctuation for the soil region. Table 5-1 gives the means, coefficient of variations, and standard deviations of the variables for each limit state using Mid-Range COVs. The simulation sets in this section were created using Mid-Range COVs, but later sections will evaluate this problem set with Upper Range COVs.

Table 5-1. Mid-Range means and standard deviations for soil effective angle of internal friction $\phi$ 'values, in degrees, for the three different limit states and their three different soil regions along with their COV.

\begin{tabular}{|c|c|c|c|c|c|c|c|c|c|}
\hline \multirow[b]{2}{*}{ Limit State } & \multicolumn{3}{|c|}{ Driving Wedge } & \multicolumn{3}{|c|}{ Structural Wedge } & \multicolumn{3}{|c|}{ Resisting Wedge } \\
\hline & mean & $\mathrm{COV}$ & $\begin{array}{l}\text { Std. } \\
\text { Dev. }\end{array}$ & mean & $\mathrm{COV}$ & $\begin{array}{l}\text { Std. } \\
\text { Dev. }\end{array}$ & mean & $\mathrm{COV}$ & $\begin{array}{l}\text { Std. } \\
\text { Dev. }\end{array}$ \\
\hline Sliding & 35 & 0.026 & 0.91 & 30 & 0.2 & 6.00 & 37 & 0.039 & 1.43 \\
\hline $\begin{array}{l}\text { Base Area in } \\
\text { Compression } \\
\text { (Overturning) }\end{array}$ & 35 & 0.026 & 0.90 & 30 & 0.2 & 6.00 & 37 & 0.041 & 1.50 \\
\hline Bearing & 35 & 0.026 & 0.91 & 30 & 0.021 & 0.63 & 37 & 0.039 & 1.43 \\
\hline $\begin{array}{l}\text { Similar } \\
\text { (average of } \\
\text { Sliding and } \\
\text { BAiC) }\end{array}$ & 35 & 0.026 & 0.90 & 30 & 0.2 & 6.00 & 37 & 0.040 & 1.47 \\
\hline
\end{tabular}


Figures 5.11 - 5-14 show simulation values drawn from each Limit States set of distributions for the three variables, forming a 3-D space. The three variables are described by the three axes shown as red, green, and blue arrows representing the orthogonal $\phi^{\prime} D W, \phi^{\prime} S W$, and $\phi^{\prime} R W$ values, respectively. The crossing point for the three axes is at the mean value origin for the distributions. These plots are shown in the non-normalized variable space so that distribution values can be compared as actual distances. Sample points are shown in either a blue color or red color representing satisfactory or unsatisfactory performance, respectively. Additionally, a green plane is shown that reveals the results of an ASM analysis for a given limit state. The ASM analysis assumes a hyper-planar response surface dividing the satisfactory response half-space from unsatisfactory response half-space, which is normal to a design point, given as the point of greatest probability on the surface. For these images, there is only a single plane shown associated with the sliding limit state in Figure 5-11. Figure 5-11 was created by overlapping the samples for all of the three Geotechnical limit states, which were simulated separately. These mean and COV values are the same as reported in Table 2-1. Because the standard deviation of the Structural Wedge $\phi$ values are so much different between the sliding and base area in compression limit states as compared to the bearing limit state, the shape of the distribution sample set varies from a long ellipsoid (Figures 5-12 and 5-13) to a vertical disk (Figure 5-14). 
Figure 5-11. Sample simulation sets of 3-D (Driving, Resisting, and Structural Wedge) material soil friction $\phi$ variable data drawn from the different sets of Normal distributions for each of the three limit states overlapped, given a T-Wall with base width of $25 \mathrm{ft}$.

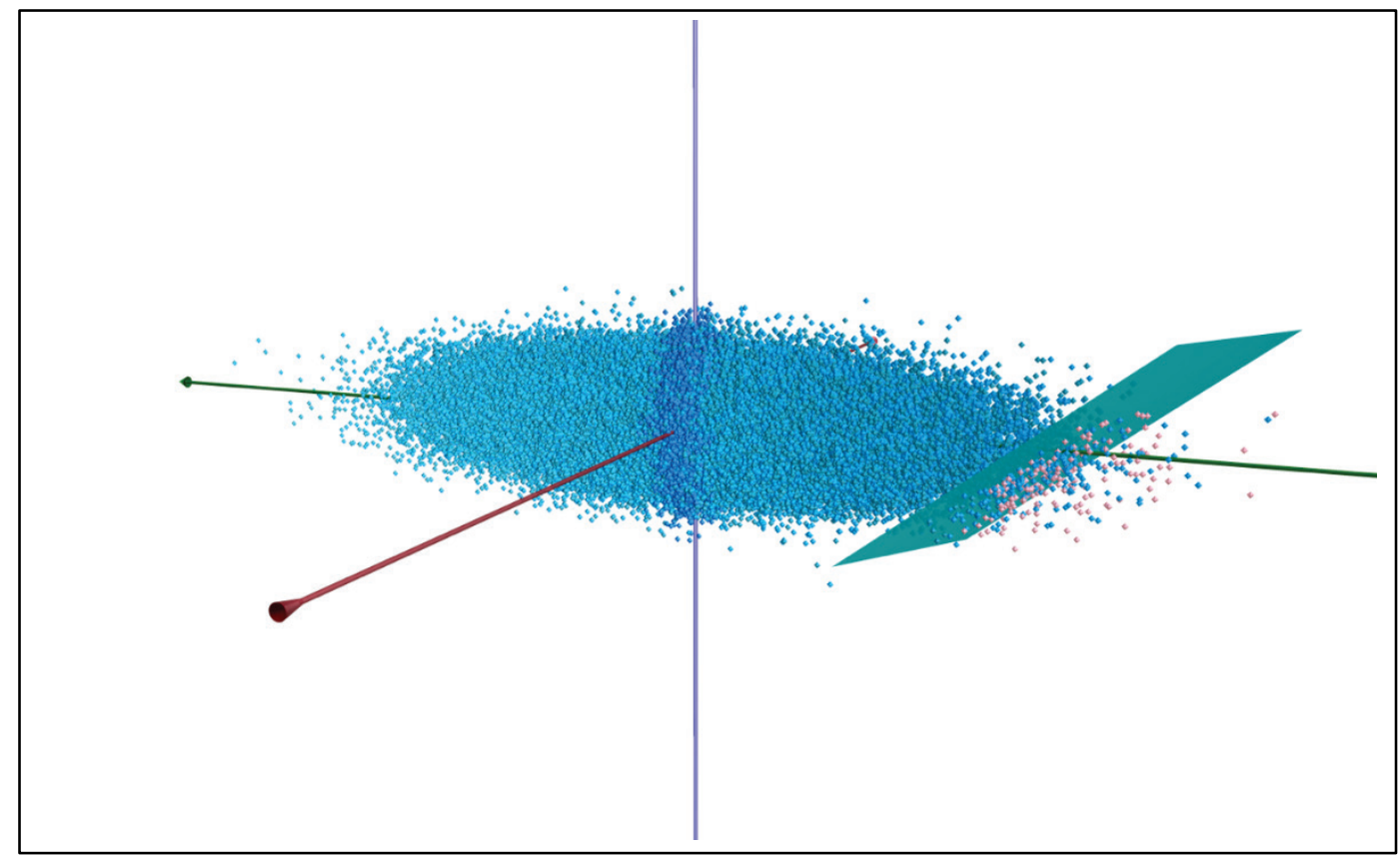

Figure 5-12. Sample set of 3-D (Driving, Resisting, and Structural Wedge) material soil friction $\phi$ variable data drawn from Normal distributions for the Base Area in Compression Limit State, given a T-Wall with base width of $25 \mathrm{ft}$.

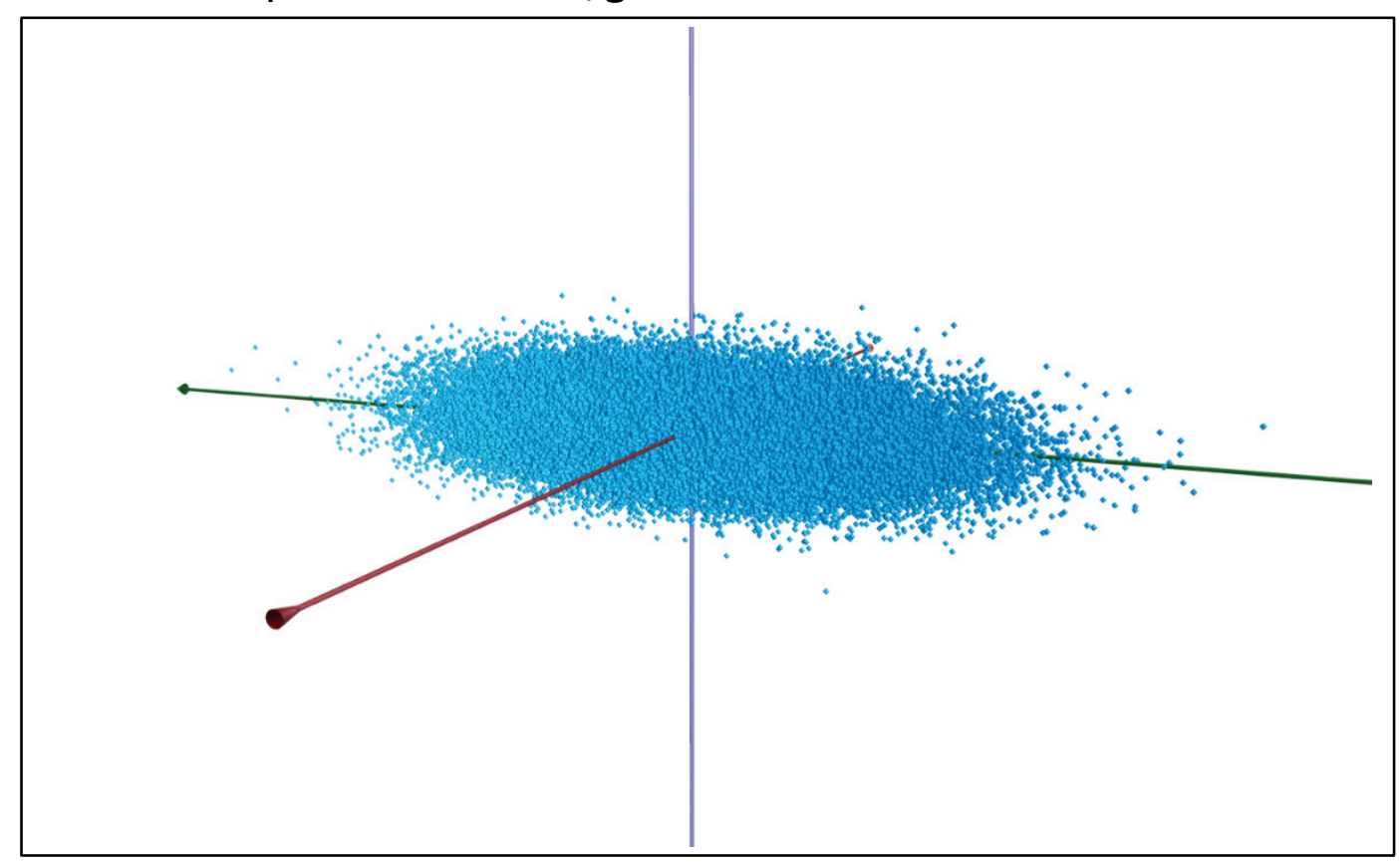


Figure 5-13. Sample set of 3-D (Driving, Resisting, and Structural Wedge) material soil friction $\phi$ variable data drawn from Normal distributions for the Sliding Limit State with Satisfactory and Unsatisfactory performance points and ASM limit state hyperplane, given a T-Wall with base width of $25 \mathrm{ft}$.

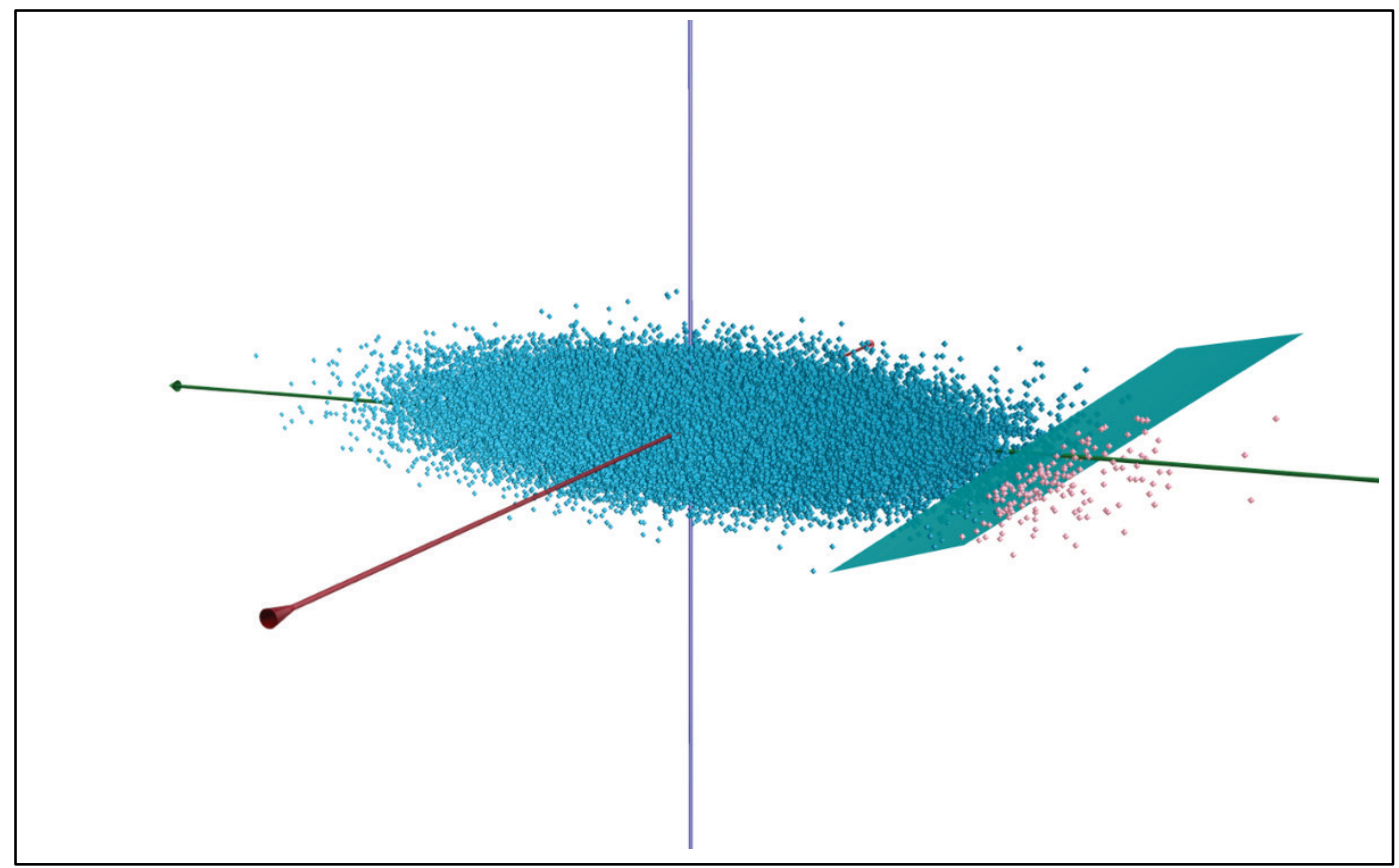

Figure 5-14. Sample set of 3-D (Driving, Resisting, and Structural Wedge) material soil friction $\phi$ variable data drawn from Normal distributions for the Bearing Limit State with no Unsatisfactory performance points, given a T-Wall with base width of $25 \mathrm{ft}$.

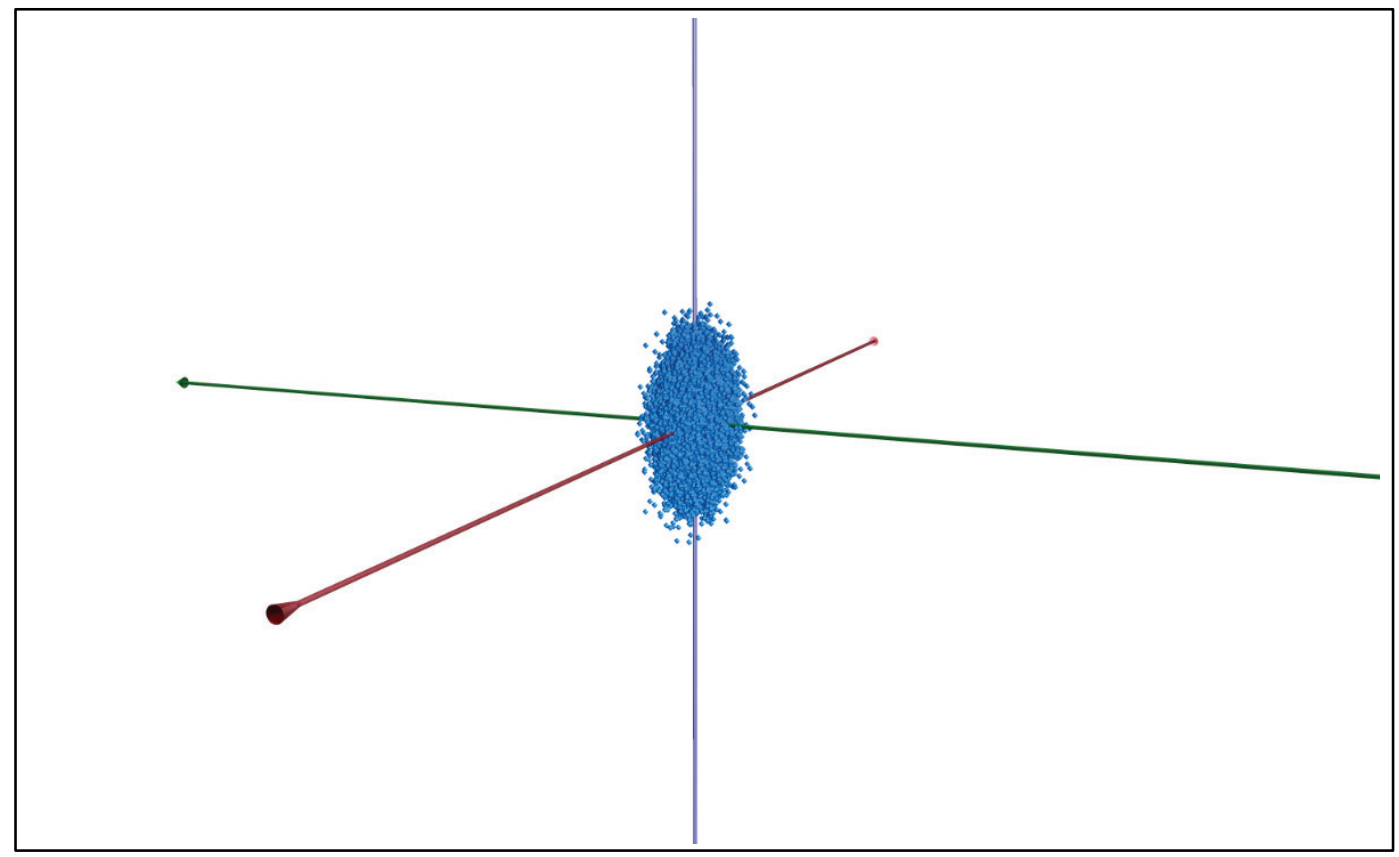


In practice, the calculated values of load and resistance forces in the simulations are found to be correlated because of the force interactions among the three wedges and the imposed force equilibrium for the three wedges. Because the Sliding and Base Area in Compression limit state slip plane distances are similar, the resulting set of variable distributions is similar, as reflected in their spatial COV values from Table 5-1. These similar distributions are further revealed in Figures 5-12 and 5-13, where the sample point ellipsoid shapes and ranges are similar. At this stage in the discussion, it is pointed out that the structural shear and moment limit states for the stem of the T-Wall, by definition, use the same set of spatial distributions as the Sliding Limit State.

The Bearing Limit State is based on vertical forces of the Structural Wedge acting on the soil foundation, resulting in a very different spatial distribution for the dispersion in the soil strength property along the slip plane for bearing failure within the foundation, beneath the structural wedge. Observe in Table 5-1 that the foundations spatial COV possesses a value that is $90 \%$ smaller than the Structural Wedge $C O V_{\text {spatial }}$ from the other two Geotechnical Limit States. This is also reflected in the simulation results when those for the Bearing limit state (Figure 5-14) are compared to those of the other two limit states (Figures 5-12 and 5-13). While the slip plane distances for the Driving and Resisting Wedges are similar to the Sliding and Base Area in Compression calculations, the Structural Wedge slip plane of a bearing failure within the foundation expanded by seven times, resulting in a $90 \%$ smaller Structural Wedge $C O V_{\text {spatial. }}$ In summary, the Bearing limit state set of soil material distributions is non-similar to the other two limit state distributions.

This difference among spatial COVs for the three limit states complicates the computation of PUP system, which combines all of the Limit State's unsatisfactory probabilities. The factors that are applied to transform the variable space to a normalized Gaussian variable space are different for the same variable for different limit states because the standard deviations are different. This violates the isotropy of this transformed space. Precise values for Monte Carlo simulations cannot be computed for all the Limit states because a drawn simulation variable value will have a different probability of selection (due to anisotropic variables) for different limit states. For example, for the sample point $\left\{\phi^{\prime} D W, \phi^{\prime} S W, \phi^{\prime} R W\right\}=\{35.0,36.0$, 37.0 $\}$ the probability of occurrence is that for 1 standard deviation $(\beta)$ for Sliding and Base Area in Compression limit states but $9.52 \beta$ for the 
Bearing limit state. This change in probability is illustrated by the shape of the drawn simulations for the Bearing limit state (Figure 5-14) as compared to the shapes of Sliding and Base Area in Compression limit states (Figures 5-12 and 5-13).

This $25 \mathrm{ft}$ wide $\mathrm{T}$-Wall example problem allows the comparison of the results of simulation to the numerical methods with those of the ASM procedure for calculating PUP for the system (of three limit states), since the primary and highest probability source of failures comes from the Sliding limit state. For 1,000,000 simulation runs, the PUP values for the Base Area in Compression and Bearing Limit states are 0.o, and the Sliding limit state PUP is 0.001669. The ASM prediction for PUP of the Sliding limit state is 0.001760 , which is overpredicting the simulation probability and indicates that the Sliding limit state response surface is non-linear. Observe in Figures 5-11 and 5-13 that the ASM limit state response surface for sliding is depicted as a plane. This 3-D surface depiction is not a precise depiction of the response surface for the reason just stated. It should have some curvature in it. Using these numbers, it can be assumed that the $P U P_{\text {system }}$ is equivalent to the Sliding limit state PUP of 0.001669.

To calculate the actual $P U P_{\text {system }}$ value, with overlapping limit states using different limit state spatial distributions, a numerical integration of the transformed (normalized Gaussian) variable space in unsatisfactory performance needs to be performed as displayed in Figure 5-4. The entire space is divided into "cells." An indicator function returns true if any limit state gives unsatisfactory performance. If multiple limit states report unsatisfactory performance for the variable values, the PDFs are computed for those limit states, and the greatest probability is returned for the cell

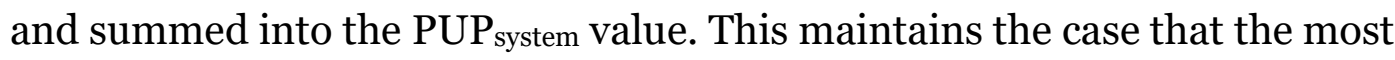
probable unsatisfactory limit state will occur before the other limit states are activated.

Appendix sections A.3.3 and A.3.4 present methods for faster numerical integration using hyperspheres, but these optimizations assume that the same spatial distribution sets are being used if multiple limit states are being solved. This is because the hypersphere method depends on normalizing the spatial distributions so that hyperspherical shells with constant probability are assumed. Refer to Figures A-10 and A-11 for a visual depiction of these multiple "shells." For the case where multiple limit states have similar spatial distributions, these limit states can be combined 
for the calculation of $P U P_{\text {system }}$ in an optimized manner. To deal with the non-similar distributions, the center of the shell element can be found in the variable space ( $\phi^{\prime} D W, \phi^{\prime} S W, \phi^{\prime}{ }^{\prime} W$ ) and the limit state evaluated for unsatisfactory performance. In Figure A-10 or A-11, each shell is divided into equal length segments around the circumference of each shell (not shown), each of which are referred to as shell cells or simply cells in this document. If the cell center is unsatisfactory for the non-similar distribution, then the probability of the cell center calculation is computed from the variable spatial distributions and, if the probability exceeds the similar distribution probability, then that value is substituted into the $P U P_{\text {system }}$ summation. This hybrid approach allows for a semi-optimized hyperspherical calculation of $P U P_{\text {system, }}$ if a smaller number of non-similar distributions exist as compared to the similar distributions.

Note that this hybrid hyperspherical PUP system method loses some precision in the calculation of the non-similar distribution probability. The hyperspherical shell elements keep the exact values for the normalized spatial distributions specifically because the distribution is normalized, which means that the curvature of the probability follows the shell. For non-similar distributions, the curvature of probability is hyperellipsoid and does not exactly match the hyperspherical shell. However, these resulting arcs of the hyperellipsoid will show symmetric properties about the center of the shell section with excess probability in one area of the shell section being cancelled by a probability reduction in the area of the shell section across the central point of the shell section.

For the $25 \mathrm{ft}$ wide T-Wall example problem of Chapter 2, there is only one truly non-similar set of distributed soil material variables which occurs for the Bearing limit state. The other limit state sets of distributions are similar enough that averaging the $C O V_{\text {spatials }}$ of the similar individual limit states will yield similar probability curvature for these limit states, especially as the tails of the distribution are approached. The Bearing limit state has a much different probability curvature about the Structural Wedge soil material variable axis. This situation is ideal for the hybrid PUPsystem calculation method suggested in the preceding paragraphs.

Figure $5^{-15}$ shows the result of performing the hybrid hyperspherical $P U P_{\text {system }}$ calculation with a target precision of 0.000001 probability. Observe in this figure that the pattern of simulation points for the shell cell centers follows a radial coordinate pattern emanating from the origin to the 
three axes (i.e., from the mean values for the three variables). The procedure generated 1,007,438 sampling runs. The blue points are satisfactory results while the green-yellow points show where the Bearing failures dominate and the red points show where Sliding failures occur. The green plane is the ASM position of the failure response surface for the sliding limit state. The linear progression of points outside of the main conglomeration of points is the area where a minimum number of points are applied to the hyperspherical shells and the probabilities are reduced.

Figure 5-15. Hybrid hyperspherical $P U P_{\text {system }}$ simulation results for a T-Wall under Extreme loads with base width of $25 \mathrm{ft}$.

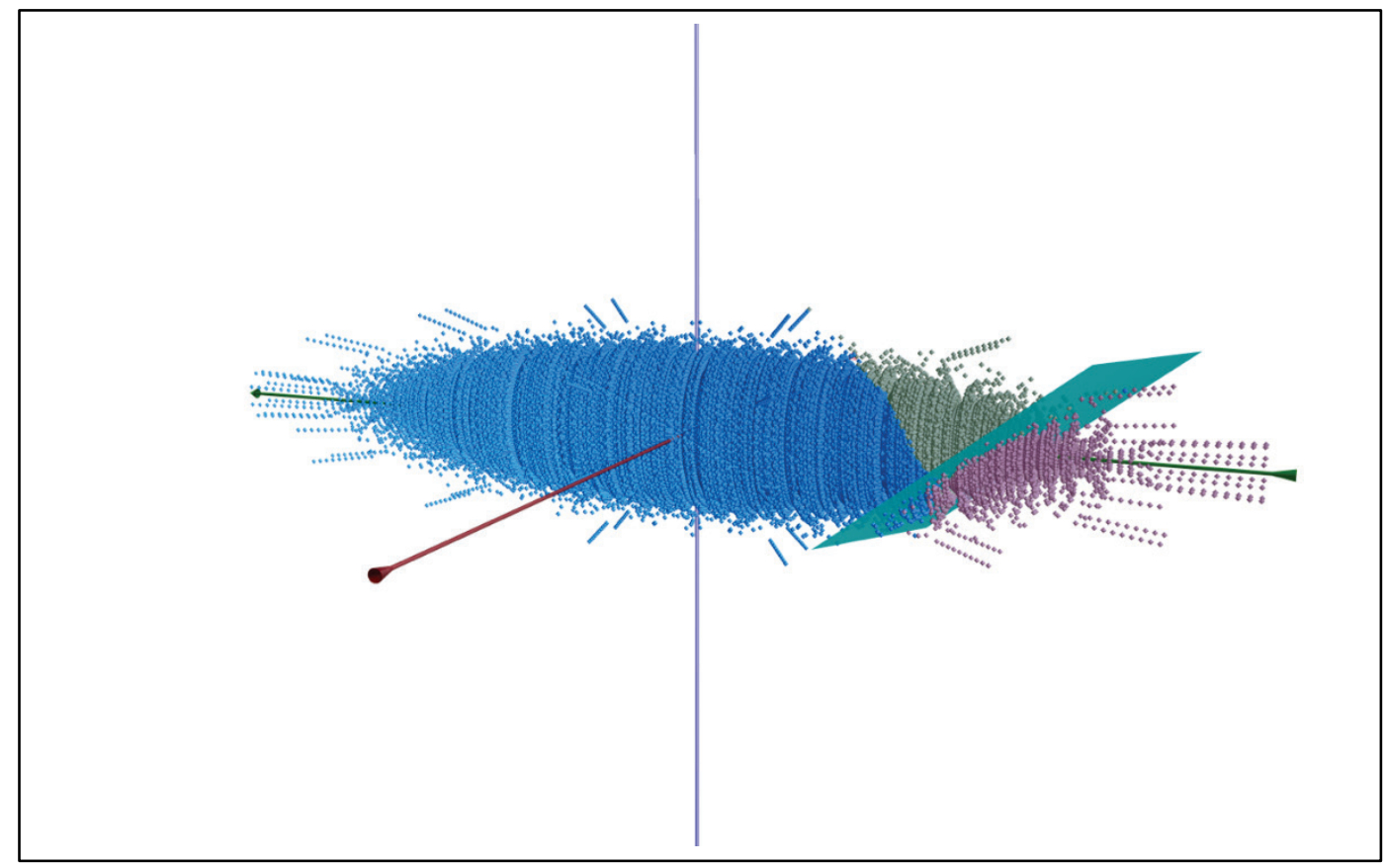

Each simulation point in Figure 5-15 has a probability of approximately 0.000001 for the similar limit states, The Bearing limit state probabilities are significantly smaller, especially as $\phi^{\prime}{ }^{\prime} w$ varies from the mean. Summing the sample point probabilities of all of the limit states, the PUP system calculated using the hybrid hyperspherical numerical method was 0.001664 .

This PUPsystem value of 0.001664 is slightly lower than the Monte Carlo simulation results for the Sliding limit state with only a 0.000005 difference. The authors of this report consider the values from the two computations to be close in magnitude. This small difference could be a result of sampling error, but it highlights that the Sliding limit state has a non-linear response surface. This nearness was expected, since the Sliding 
limit state was the primary concern for failures. It is observed that the extremely low probabilities of the Bearing limit state failure conditions made no substantive difference in the $P U P_{\text {system }}$ calculation. While this condition allows for a nearly direct comparison of $P U P_{\text {system }}$ to $P U P_{\text {Sliding }}$ for verification, it does not allow us to see the results of LRFD analysis as compared to individual limit state LRFD analysis. An additional analysis is required to address this feature.

Applying Equation 5.14 to the PUP system value of 0.001664 for the $25 \mathrm{ft}$ base problem, the resultant equivalent normal $\beta_{\text {system value used as }}$ approximation for system Reliability is 2.94 .

Figure 5-16 shows the results of running the Usual load case (from Figure 2-2) with the base width reduced to $15 \mathrm{ft}$. This case was chosen because the ASM runs for Sliding, Base Area in Compression, and Bearing all resulted in failures with significant (albeit low) probabilities. The three planes are visible, with the Bearing limit state response surface (dark blue) plane being above the mass of the samples, the Base Area in Compression limit state response surface (turquois) plane being behind the mass of the samples, and the Sliding limit state response surface (dark green) plane proceeding at an angle across the front, left of the mass of the samples. The ASM PUP for the Base Area in Compression limit state is still extremely small and does not register to six decimal places. The ASM $P U P$ for the Bearing limit state is not insignificant with a value of 0.00032. The ASM PUP for the Sliding limit state still dominates with a value of $0.01075^{2}$. PUP $P_{\text {system }}$ from the hybrid hyperspherical numerical procedure had a value of 0.011857 . Again, it is observed that the sliding limit state dominates the contribution to $P U P_{\text {system }} 25 \mathrm{ft} \mathrm{T}$-Wall base width example, and that both the Bearing and the Base Area in Compression limit states provide negligible contributions. 
Figure 5-16. Hybrid hyperspherical $P U P_{\text {system }}$ simulation results for a T-Wall under Usual loads with base width of $15 \mathrm{ft}$.

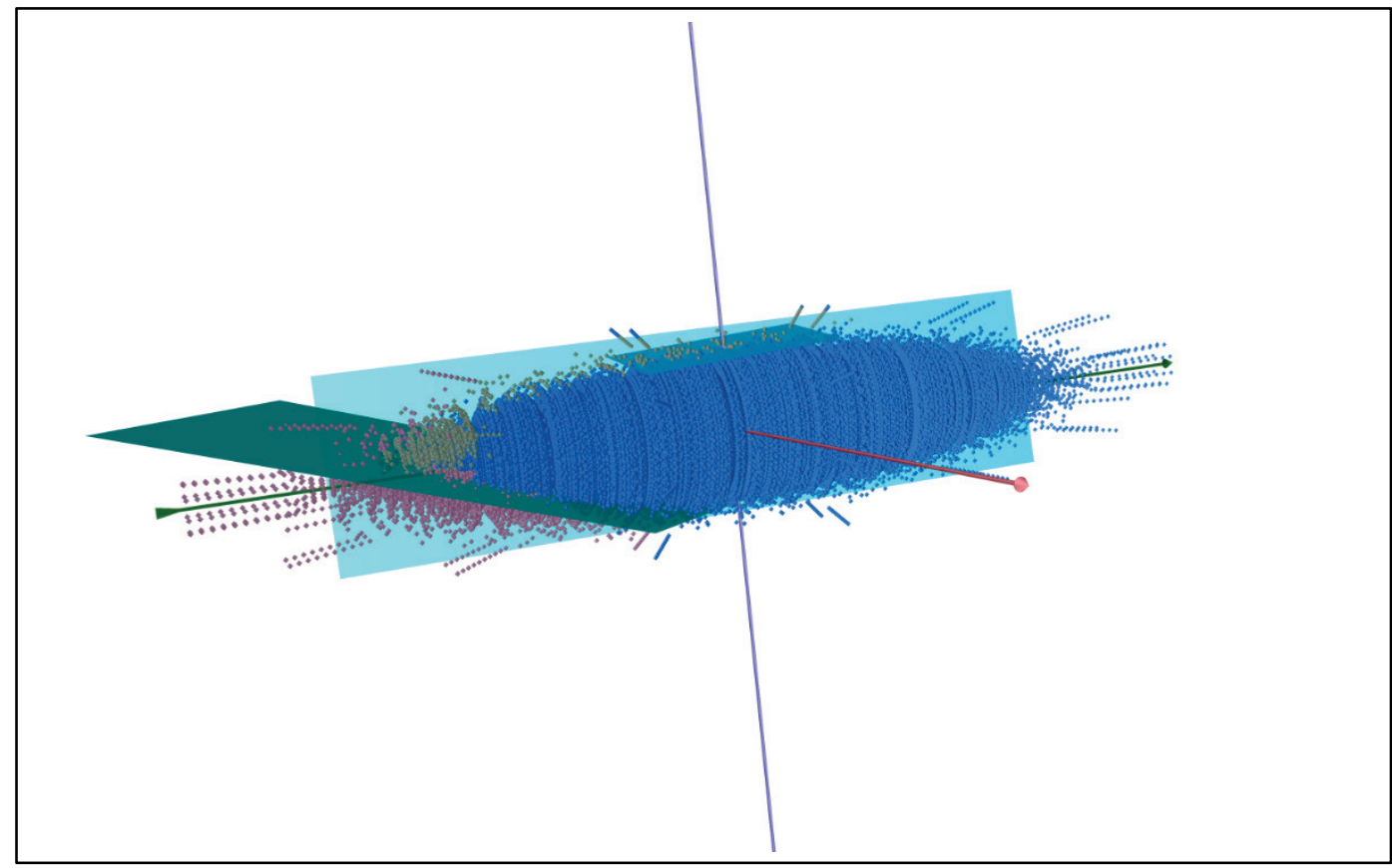

This case was included to show why numerical integration is necessary. The ASM plane does not begin to describe the non-linear response of the Bearing limit state. As samples are drawn that have very low $\phi^{\prime} s w$ values, there is less support for the structure so that less vertical force from the driving soil wedge is needed to put the structure into failure, leading to an asymptotic curve to the response surface. Due to the scale of fluctuation and the slip plane length for the mobilized soil under the Bearing load case, the probability of very low $\phi^{\prime} s w$ values is extremely small.

In conclusion, it is shown that limit state response surfaces are non-linear and overlapping and that a full integration of maximum probabilities will give a more precise calculation of $P U P_{\text {system. }}$. It is also shown that the adaptive hyperspherical Gaussian probability numerical integration will allow for high precision calculation of $P U P_{\text {system }}$ using a similar number of simulation runs (approximately 1,000,000 per limit state for a precision of 0.000001) as Monte Carlo methods. Unfortunately, the probabilities for the base design example problem is driven primarily by the Sliding limit state. The $15 \mathrm{ft}$ wide T-Wall example provides a method to view all of the limit state ASM hyperplanes and explicitly reveals the nonlinearity of the bearing limit state. While this condition allows for a nearly direct comparison of PUPsystem to PUPSliding for verification, it does not allow one to see the results of LRFD analysis as compared to individual limit state 
LRFD analysis. An additional analysis method is required to address this feature.

Applying Equation 5.14 to the PUP system value of 0.011857 for the $15 \mathrm{ft}$ wide base problem, the resultant equivalent normal $\beta_{\text {system value used as }}$ approximation for system Reliability is $\mathbf{2 . 2 6}$.

\subsection{Calculating PUP $P_{\text {system }}$ for the Chapter 2 T-Wall Example Problem with Geotechnical and Structural Limit States using $25 \mathrm{ft}$ base width}

In the previous section, $P U P_{\text {system }}$ results for only the three geotechnical limit states were evaluated to reveal the non-linearity or non-planarity of the response surface boundary between the satisfactory and unsatisfactory performance regions. Performing only three limit states also made it simpler to visualize the complete results which only have three dimensions. This section extends the previous section with two more structural limit states.

The limit states of the previous section could be described by a function of three variables that returned the status of the limit state, either satisfactory or unsatisfactory. These three variables were the friction angles of the soil along the slip planes contained within the Driving, Structural, and Resisting soil wedges ( $\phi_{D W}^{\prime}, \phi_{S w}^{\prime}$, and $\phi_{R W}^{\prime}$ ). The distributions for these variables were computed based on point values for the material coefficient of variation and application of the variance reduction factor computed using the length of the slip planes and the scale of fluctuation for the soil materials.

The three Limit State example problem can be extended to include structural limit states such as shear forces and moments acting upon the vertical section of the wall, commonly referred to as the stem. The additional two structural limit states use the same sets of soil strength variables but use them to compute the total shear force or the total moment acting at the base of the stem of the wall feature on the T-Wall. The distributions for these variables use the same computations that are used for the Sliding Limit State, calculating the same variance reduction factors computed based on the slip plane calculations from Section 3.4 for the mean variable values. Therefore, these distributions for the geotechnical soil materials are similar to the Sliding and Base Area in Compression Limit States.

These computations are discussed in Sections 3.6 and 3.7 as well as Appendix B. The resulting shear load or moment becomes the load on the 
system. Each structural limit state has another variable that is a directly computed shear or moment resistance. The capacity for the Extreme load case is dictated by the Usual load case demand multiplied by a load factor of 2.2 according to EM 1110-2-2104 (HQUSACE 2016) Table 3-1 value for the Serviceability limit state. For the Usual load case, the shear demand at the base of the stem is computed to be $17,024 \mathrm{lb}$, and the moment demand is computed to be $228,612 \mathrm{ft}-\mathrm{lb}$.

Table 3-9 for shear capacity and Table 3-10 for flexural capacity give distribution type, bias factors for mean, and coefficient of variation as well as standard deviations that define the capacity distributions for shear and moment according to Ellingwood. The mean capacity is therefore the serviceability capacity times the bias factor for the mean, with the standard deviation of the capacity being the COV times the mean capacity. Because Ellingwood defined both structural capacity distribution types to be a lognormal distribution, each variable can be drawn from a normalized Gaussian distribution and converted to a lognormal distribution value.

This means that the final two structural limit states status calculations are functions of four variables. The stem shear limit state is a function with $\phi_{D W}^{\prime}, \phi_{S W}^{\prime}, \phi_{R W}^{\prime}$, and the shear force resistance of the stem $\left(S_{R}\right)$. The stem moment limit state is a function with $\phi^{\prime} D w, \phi^{\prime} s w, \phi_{R W}^{\prime}$, and the moment resistance of the stem $\left(S_{M}\right)$. The introduction of $S_{R}$ and $S_{M}$ means that the adaptive hyperspherical Gaussian probability numerical integration procedure will have to be extended from 3-D to five-dimensional (5-D), since the number of distributed variables has increased. It is incidental that the number of limit states is 5 , also.

The new variables only affect one limit state apiece. Only distributed variables that are shared between limit state functions need to be compared for similarity because a function that does not use a variable will not be affected by changes of the variable. Because the only shared variables of the two new structural limit states are the $\phi_{D W}^{\prime}, \phi_{S W}^{\prime}$, and $\phi_{R W}^{\prime}$ values, which are determined in the same fashion as the Sliding values, these limit states are similar to each other and the Sliding and Base Area in Compression Limit State. The lone dissimilar set of limit state distributions are found for the Bearing limit state, as it was for the 3-D numerical analysis. The distributions of the geotechnical variables with Mid-Range and Upper Range COVs are shown in Tables 5-2 and 5-3, respectively. The structural Limit State distributions are shown in Table 5-4. 
Table 5-2. Mid-Range means and standard deviations for soil effective angle of internal friction $\phi^{\prime}$ 'values, in degrees.

\begin{tabular}{|c|c|c|c|c|c|c|c|c|c|}
\hline \multirow{3}{*}{ Limit State } & \multicolumn{9}{|c|}{ Mid-Range COV values } \\
\hline & \multicolumn{3}{|c|}{ Driving Wedge } & \multicolumn{3}{|c|}{ Structural Wedge } & \multicolumn{3}{|c|}{ Resisting Wedge } \\
\hline & $\begin{array}{l}\text { mean } \\
\text { degrees }\end{array}$ & $\mathrm{COV}$ & $\begin{array}{l}\text { std. dev. } \\
\text { degrees }\end{array}$ & $\begin{array}{l}\text { mean } \\
\text { degrees }\end{array}$ & $\mathrm{COV}$ & $\begin{array}{l}\text { std. dev. } \\
\text { degrees }\end{array}$ & $\begin{array}{l}\text { mean } \\
\text { degrees }\end{array}$ & $\mathrm{COV}$ & $\begin{array}{l}\text { std. dev. } \\
\text { degrees }\end{array}$ \\
\hline Sliding & 35 & 0.026 & 0.91 & 30 & 0.2 & 6.00 & 37 & 0.039 & 1.43 \\
\hline $\begin{array}{l}\text { Base Area in } \\
\text { Compression } \\
\text { (Overturning) }\end{array}$ & 35 & 0.026 & 0.90 & 30 & 0.2 & 6.00 & 37 & 0.041 & 1.50 \\
\hline Bearing & 35 & 0.026 & 0.91 & 30 & 0.021 & 0.63 & 37 & 0.039 & 1.43 \\
\hline Stem Shear & 35 & 0.026 & 0.91 & 30 & 0.2 & 6.00 & 37 & 0.039 & 1.43 \\
\hline Stem Moment & 35 & 0.026 & 0.91 & 30 & 0.2 & 6.00 & 37 & 0.039 & 1.43 \\
\hline $\begin{array}{l}\text { Similar (average } \\
\text { of Sliding, BAiC, } \\
\text { Stem Shear, } \\
\text { and Stem } \\
\text { Moment) }\end{array}$ & 35 & 0.026 & 0.91 & 30 & 0.2 & 6.00 & 37 & 0.039 & 1.45 \\
\hline
\end{tabular}


Table 5-3. Upper Range means and standard deviations for soil effective angle of internal friction $\phi$ 'values, in degrees.

\begin{tabular}{|c|c|c|c|c|c|c|c|c|c|}
\hline \multirow{3}{*}{ Limit State } & \multicolumn{9}{|c|}{ Upper Range COV Values } \\
\hline & \multicolumn{3}{|c|}{ Driving Wedge } & \multicolumn{3}{|c|}{ Structural Wedge } & \multicolumn{3}{|c|}{ Resisting Wedge } \\
\hline & $\begin{array}{l}\text { mean } \\
\text { degrees }\end{array}$ & COV & $\begin{array}{l}\text { std. dev. } \\
\text { degrees }\end{array}$ & $\begin{array}{l}\text { mean } \\
\text { degrees }\end{array}$ & COV & $\begin{array}{l}\text { std. dev. } \\
\text { degrees }\end{array}$ & $\begin{array}{l}\text { mean } \\
\text { degrees }\end{array}$ & $\mathrm{COV}$ & $\begin{array}{l}\text { std. dev. } \\
\text { degrees }\end{array}$ \\
\hline Sliding & 35 & 0.052 & 1.82 & 30 & 0.3 & 9.00 & 37 & 0.077 & 2.85 \\
\hline $\begin{array}{l}\text { Base Area in } \\
\text { Compression } \\
\text { (Overturning) }\end{array}$ & 35 & 0.051 & 1.79 & 30 & 0.3 & 9.00 & 37 & 0.081 & 3.00 \\
\hline Bearing & 35 & 0.052 & 1.82 & 30 & 0.032 & 0.95 & 37 & 0.077 & 2.85 \\
\hline Stem Shear & 35 & 0.052 & 1.82 & 30 & 0.3 & 9.00 & 37 & 0.077 & 2.85 \\
\hline Stem Moment & 35 & 0.052 & 1.82 & 30 & 0.3 & 9.00 & 37 & 0.077 & 2.85 \\
\hline $\begin{array}{l}\text { Similar (average } \\
\text { of Sliding, BAiC, } \\
\text { Stem Shear, } \\
\text { and Stem } \\
\text { Moment) }\end{array}$ & 35 & 0.052 & 0.91 & 30 & 0.3 & 9.00 & 37 & 0.078 & 2.89 \\
\hline
\end{tabular}

Table 5-4. Means and standard deviations for stem shear force and stem moment.

\begin{tabular}{|c|c|c|c|c|c|c|}
\hline \multirow[b]{2}{*}{ Limit State } & \multicolumn{3}{|c|}{ Stem Shear Force } & \multicolumn{3}{|l|}{ Stem Moment } \\
\hline & $\begin{array}{l}\text { Mean } \\
\mathrm{GN}^{*}\left(\mathrm{LN}^{* *}\right)\end{array}$ & $\mathrm{COV}$ & $\begin{array}{l}\text { Std. Dev. } \\
\mathrm{GN}^{*}\left(\mathrm{LN}^{* *}\right)\end{array}$ & $\begin{array}{l}\text { Mean } \\
\mathrm{GN}^{*}\left(\mathrm{LN}^{* *}\right)\end{array}$ & COV & $\begin{array}{l}\text { Std. Dev. } \\
\mathrm{GN}^{*}\left(\mathrm{LN}^{* *}\right)\end{array}$ \\
\hline Stem Shear & $\begin{array}{l}10.654 \\
(43,070.72 \\
\text { lb) }\end{array}$ & 0.0168 & $\begin{array}{l}0.179 \\
(7,752.73 \\
\text { lb) }\end{array}$ & & & \\
\hline $\begin{array}{l}\text { Stem } \\
\text { Moment }\end{array}$ & & & & $\begin{array}{l}13.232 \\
(563,299.97 \\
\mathrm{ft}-\mathrm{lb})\end{array}$ & 0.0105 & $\begin{array}{l}0.139 \\
(78,862.00 \\
f t-l b)\end{array}$ \\
\hline
\end{tabular}

* Gaussian Normal distribution value (designated GN) to be converted to actual Log-Normal value

** Resultant actual Log-Normal distribution value (designated LN)

Table 5-2 has the same form and mostly the same values of Table 5-1. Table 5-3 gives the Upper Range distributions for these same variables. The introduction of the new Stem Shear and Stem Moment distributions, which are calculated in the same fashion as the Sliding Limit state and therefore have the same values, makes a slight adjustment in the similar distribution when averaging occurs.

Table 5-4 has the distributions of the new variables introduced for the Stem Shear and Stem Moment limit states. The mean and standard deviation 
values for the log-normally distributed shear force and moment variables. The variables are then converted to a Gaussian Normal distribution that, when used as the exponent $x$ in equation $e^{X}$, derives the lognormal value required for the variable. The Gaussian Normal distributions created for stem shear and moment are used as the two new axes in the 5-D variable coordinate system.

An ASM run was performed for each limit state to find the individual limit state PUP value, estimated using a hyperplanar response surface normal to a design point. Table 5-5 shows the results for both Mid-Range and Upper Range COV distributions, with N/A results occurring where the ASM bounds for $\beta$ were exceeded ( $>8$ ). Thus, the N/A cases possess a very low $P U P$ value with a corresponding $\beta$ value greater than 8 .

Table 5-5. ASM results for the limit states of the 5-D integration problem.

\begin{tabular}{|l|l|l|l|l|}
\hline \multirow{2}{*}{ Limit State } & \multicolumn{2}{|l|}{ Mid-Range COVs } & \multicolumn{2}{l|}{ Upper Range COVs } \\
\cline { 2 - 5 } & $25 \mathrm{ft}$ base width model & \multicolumn{2}{l|}{$25 \mathrm{ft}$ base width model } \\
\cline { 2 - 5 } & PUP & Equivalent $\beta$ & PUP & Equivalent $\beta$ \\
\hline Sliding & 0.001745170794 & 2.92 & 0.030312881586 & 1.88 \\
\hline $\begin{array}{l}\text { Base Area in } \\
\text { Compression }\end{array}$ & N/A & N/A & N/A & N/A \\
\hline Bearing & 0.000000000630 & 6.07 & 0.000235387940 & 3.49 \\
\hline Stem Shear & 0.000815793637 & 3.15 & 0.001114960718 & 3.06 \\
\hline Stem Moment & 0.000000005464 & 5.71 & 0.000000027044 & 5.44 \\
\hline
\end{tabular}

A comparison is made between the Mid-Range COV and Upper Range COV values used in the simulation runs for individual limit state performance. The expectation was that using Upper Range COV variances would increase the PUP for every limit state, which seems to be borne out for the cases for which there are data. It can be assumed that the Base Area in Compression Limits State has an increased PUP value, though that value is in the tails of the distribution. A surprising result of the comparisons is in the amount of difference that the change of variance makes for some limit states with respect to others. The Stem Shear and Stem Moment Limit States had decreases of $\beta$ of $3 \%$ to $5 \%$, the change of Sliding Limit State $\beta$ was $36 \%$, while the change of the Bearing Limit State $\beta$ was $43 \%$. It is primarily assumed by the authors of this report that these changes are primarily explained away by the Three-Wedge geotechnical method and how the foundation soil in the Structural Wedges interacts with the solution. The 
foundation soil does not apply to the Stem Shear and Stem Moment Limit States but has a slip plane length equivalent to the length of the base without separation for the Sliding Limit State and a slip plane length seven times the length of the base for Bearing. Applying the Variance Reduction to these slip plane lengths makes for a very tight distribution, so PUP values in the tails of the distribution have more significant contributions when the distribution is scaled.

Using Mid-Range COVs, the 5 -D numerical processing runs for the $25 \mathrm{ft}$ base width example T-Wall problem produced a PUP system value of 0.002374 as compared to the 3 -D numerical processing probability for the geotechnical issues alone with a value of 0.001664 . This is a significant change and indicates that the failure response surface for the system has been changed by the addition of the structural limit states. Thus, the additional consideration of the structural limit states, most probably the stem shear limit state, contributes to the PUP for the T-Wall.

Recall that the $0.001664 P U P_{\text {system }}$ was, for all intents and purposes, equivalent to the $P U P_{\text {Sliding }}$ for the $25 \mathrm{ft}$ base width example problem for the 3-D results (i.e., Geotechnical limit states). Despite non-linearities, these values were also very near to the ASM calculation for PUPSliding.

Equation 5.15 shows that the probability of two (or more) mutually exclusive limit states is equal to the sum of each of the individual limit state probabilities (Ang and Tang 1984). Assuming the probabilities for Sliding, Stem Shear, and Stem Moment are mutually exclusive, the maximum value of the PUP system can be computed by summing their probabilities. This equation can be derived from the equation for the maximum bounds given in Appendix A section A.3.1.

$$
P\left(L S_{1} \cup L S_{2}\right)=P\left(L S_{1}\right)+P\left(L S_{2}\right)
$$

Summing the PUP values for each of the limit state ASM probabilities of the $25 \mathrm{ft}$ base width example problem in Table 5-4 gives a value of 0.002596, which is close to 0.002374 , the numerical calculation of the 5-D PUP system. This would seem to suggest that the PUP volumes in the Gaussian Normal Space for each limit state do not overlap, but that is not the case.

For the Stem Shear and Moment limit states, Gaussian Normal space variable axes are created that explicitly define resistance, but given the 
definition of the Gaussian Normal distribution space, the other variable axes are orthogonal to the resistance variable axes. The Geotechnical limit state volumes are unaffected by the resistance variables. The Geotechnical hyperplanes that define ASM probabilities are therefore parallel to the axes that have no effect on them whereas the Structural limit states must not be parallel to these axes since they define performance. This leads to the conclusion that there must be overlap of the PUP volumes between the Geotechnical and Structural limit states.

This conclusion is further proven by finding the dihedral angle between the unit $\alpha$ vectors to the design points of the response surface hyper planes for the Sliding and Stem Shear limit states determined using ASM. The dihedral angle of these vectors is also the dihedral angle between the hyperplanes. The Sliding limit state $\alpha$ is the vector [Driving Wedge $\phi^{\prime}=$ 0.094745, Structural Wedge $\phi^{\prime}=0.949978$, Resisting Wedge $\phi^{\prime}=0.297598$, Stem Shear Resistance $=0.0]$. The stem shear resistance does not have bearing on the sliding limit state, so its value is set to o.o. The Stem Shear limit state $\alpha$ is the vector [Driving Wedge $\phi^{\prime}=0.134587$, Structural Wedge $\phi^{\prime}=0.0$, Resisting Wedge $\phi^{\prime}=0.048451$, Stem Shear Resistance $=$ $0.989717]$. The dot product of these vectors yields the cosine of the dihedral angle of 0.02717 , which is a dihedral angle of more than 88 deg between the hyperplanes.

The fact that the dihedral angles are virtually perpendicular and yet the $P U P_{\text {system }}$ is similar to the sum of $P U P_{\text {sliding }}$ and $P U P_{\text {stemshear }}$ leads the authors to conclude that the probability of the overlap region, where the combination of variables leads to failure in more than one limit state, is statistically insignificant in this case. This situation will occur with any overlap region (even with large dihedral angles) when the overlap is in the tails of the distributions, because the probability of any point in the variable space is the multiplication of all of the variable's probability density functions at that point. Integrating over the overlap space returns a very small number. Returning to the example problem, the greatest probability

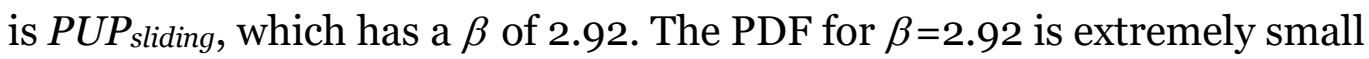
and will be multiplied by even smaller values for the next closest limit state $\beta$ (Stem Shear). Integrating the overlap space contributes an insignificant amount to PUP system.

Using Upper Range COVs creates a wholly different dynamic because the change in variance changes the contributions of the individual Limit States. 
Table 5-5 ASM analysis reveals that the increase in variation affects the Bearing and Sliding Limit States the most, respectively. While the Bearing Limit State had hardly been a concern for the Example Problem using MidRange COVs, it has become an issue for the Upper Range COVs.

Figure 5-17 shows the effect on the three primary individual limit states (Sliding, Stem Shear, and Bearing) as the resistance scale factor $\left(R_{S F}\right)$ is increased. The minimal $\beta$ point at any $R_{S F}$ location gives the greatest individual PUP that will affect the structure. Because the Stem Shear Limit State has higher $\beta$ values than the other two limit states, it is not the limit state of most likely failure.

Figure 5-17. Individual limit state analysis of $\beta$ with respect to increasing $R_{S F}$.

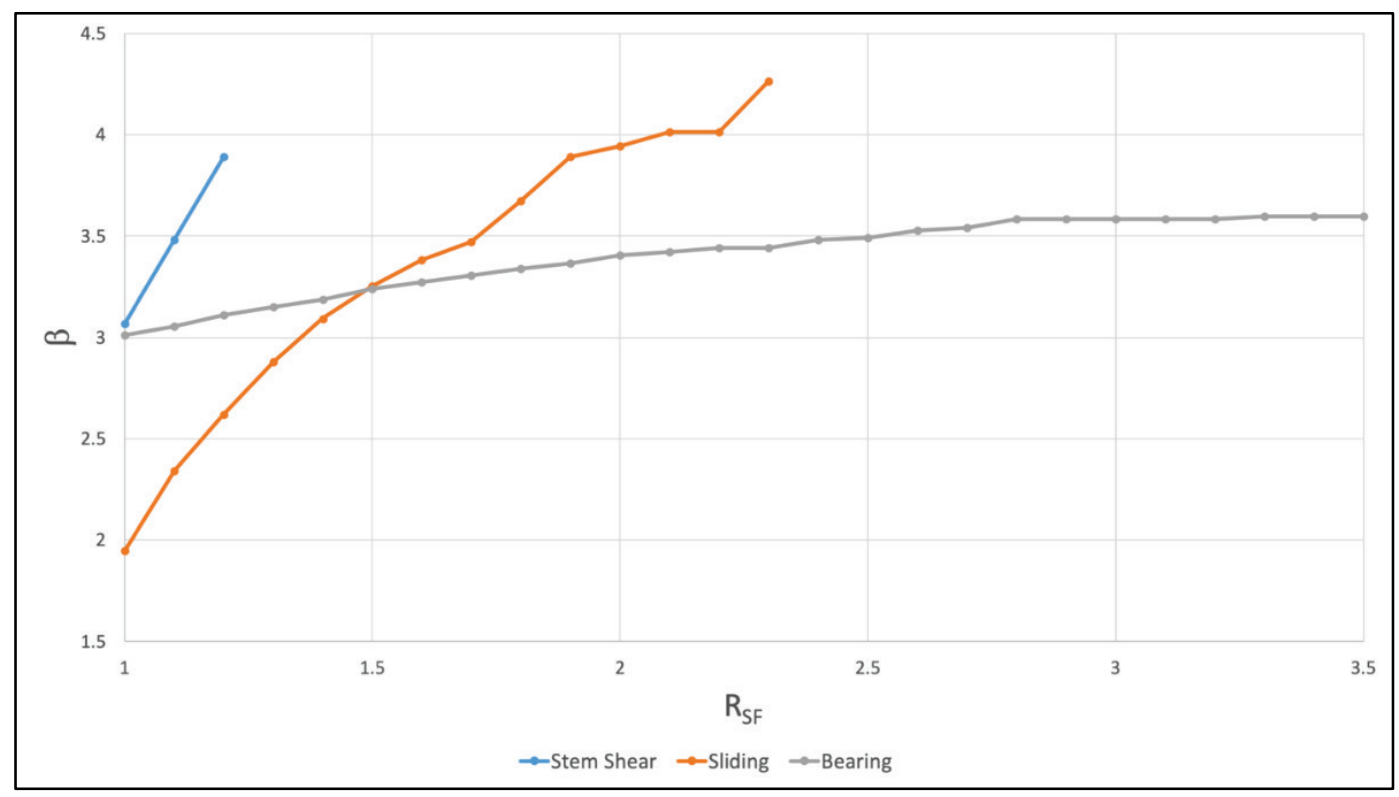

The shapes and positions of the Load and Resistance distributions for the individual limit states have a large effect on the rate of change of the individual PUPS and $\beta \mathrm{s}$ for the limit states as $R_{S F}$ changes. This is illustrated by the different slopes for the Sliding Limit State and Bearing Limit State $R_{S F}$-to- $\beta$ curves. Slight changes of $R_{S F}$ have a larger effect on $\beta$ for the Sliding Limit State than the Bearing Limit State. This leads to a situation where the most probable failure is with the Sliding Limit State until the $R_{S F}$ reaches a value of close to 1.5, and then the Bearing Limit State becomes the limit state of most probable failure. Because of the slow rate of change, high values of $R_{S F}$, meaning greater Load and Resistance Factors, are required to reach target $P U P$ and consequent $\beta$ values. For 
Figure $5^{-17}$, an $R_{S F}$ value of 2.5 is required to provide a guarantee $\beta$ of 3.5, which is recommended for noncritical structures by Ellingwood.

Figure 5-18 shows $b$ as a function of $R_{S F}$ for three different cases: simulated Sliding Limit State computations, Sliding Limit State 5-D integration, and a Combined Limit State 5-D integration. The first two cases were provided as a verification step since the simulation and 5 -D integration should provide nearly the same values, which they did. The black curve provides the result of the Combined Limit State. This value is provided by a $\mathrm{Gz}()$ function, which combines the results of the $G z()$ functions of the other Limit States. Recall that the $G z()$ function returns the Resistance minus the Load $(R-L)$. If any individual Limit State $\mathrm{Gz}($ ) function drops below o, then the $P U P_{\text {System }}$ is increased by the greatest limit state failure probability.

Figure 5-18. Comparison of simulated Sliding Limit State, 5-D integration analysis for Sliding Limit State, and 5-D integration analysis with the combined limit state response $\beta$ values as $R_{S F}$ is increased.

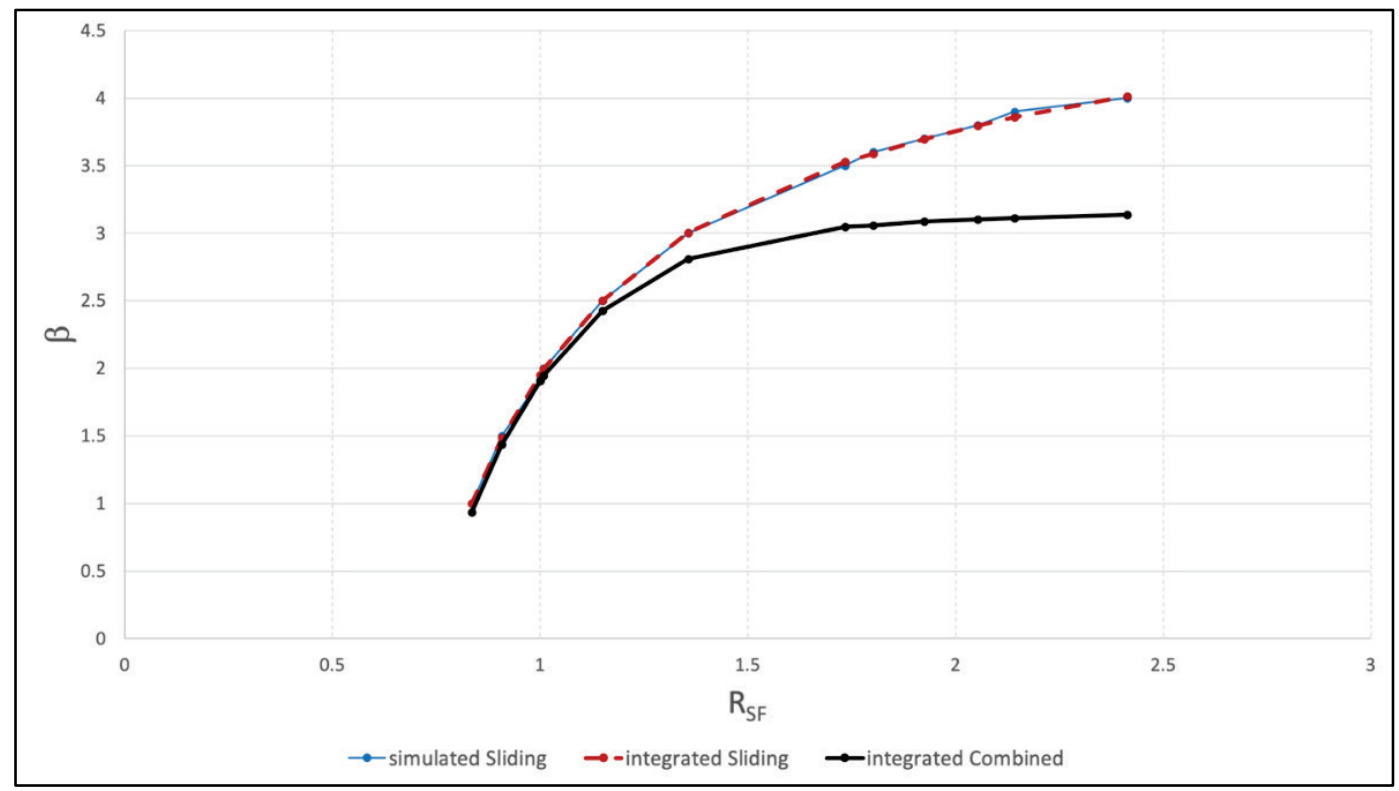

The Combined curve closely follows the Sliding PUP curve until the $R_{S F}$ factor increases to nearly 1.25 . This corresponds to the Figure 5-16 individual limit state results. The curve then starts to see the influence of the other limit states, presumably Bearing according to Figure $5^{-16}$. However, the value of $\beta$ is less than the individual $\beta$ values as $R_{S F}$ changes because of the additive effects of the overlapping failure regions for each limit state. For $R_{S F}=2.0$, the Bearing Limit State alone returns an approximate value of $\beta=3.4$ (Figure 5-16), yet the Combined Limit State returns an approximate value of $\beta=3.1$ (Figure 5-17). 
This analysis underscores the observations of Fan et al. (2014), who indicated that determining individual load and resistance factors may not provide a sufficient $P U P_{\text {System }}$ for the proposed lifetime of the structural and geotechnical pile system. Fan et al. (2014) based their inference on the fact that the probability areas of the combined limit states can be greater than the probability area of any individual limit state, as shown in the comparison of Figures 5-17 and 5-18. The observation is further bolstered by the discovery that the primary individual limit state for determining the $R_{S F}$ based on target $P U P$ and $\beta$ values can change as the $R_{S F}$ changes.

\subsection{Checking sliding resistance scale factors against the five-limit state probability integration calculation}

A comparison can be made for the Load and Resistance factors computed in Chapter 4 against the multiple limit state adaptive hyperspherical Gaussian probability numerical integration of Section 5.3.2.3 of this chapter using Upper Range COVs. This comparison serves two purposes:

(1) to show the cumulative effect of multiple limit states on PUP values and (2) to reveal whether load and resistance factors determined for the Limit State with the greatest PUP value can be used to as good baseline values. In the Extreme example problem of Figure 4-3b, the limit state that had the highest PUP value of 0.00176 was the Sliding Limit State, followed by the Stem Shear Limit State with value of 0.000836.

Simulation methods introduced in Chapter 4 provided methods for determining a limit state's resistance scale factor, $R_{S F}$, which can be applied to the resistance to determine a design resistance. When this $R_{S F}$ is applied to the specific design, the resulting PUP should be equal to a $P U P_{\text {Target. }}$ The $R_{S F}$ is converted to the $L_{S F}$ based on the mean values of the current simulation and the results are shown in Figure 4-7. The $L_{S F}$ values can then be decomposed into Load and Resistance Factors for LRFD with results shown in Figure 4-8. Table 5-5 shows the $\beta$ Target values, $R_{S F}$ values, and $L_{S F}$ values that were computed for the Sliding Limit State of the Upper Range COVs in Figure 4-7.

To verify the correctness of using the Load and Resistance values based on Sliding for the structural system being analyzed, we must apply the $R_{S F}$ to the adaptive hyperspherical Gaussian probability numerical integration procedure, because this value is tuned to the Sliding load and resistance means applied in the simulations. The $R_{S F}$ is applied to the other four 
limit state resistance values as well, so that the full system performance can be measured.

Table 5-6 shows the result of applying the Sliding $R_{S F}$ in the adaptive hyperspherical Gaussian probability numerical integration scheme in the last two columns. Observe that the $\beta_{\text {Target }}$ for Sliding exceeds the actual $\beta$ System and that the difference becomes greater as the $\beta$ Target increases. This observation is concerning because it reveals that Load and Resistance factors based on the dominant limit state (Sliding for the example problem) may be inadequate to specify designs that improve system response to a specified probability of performance.

Table 5-6. The results of applying the 5-D adaptive hyperspherical Gaussian probability numerical integration technique with the $R_{S F}$ values determined for Sliding $\beta_{\text {Target }}$ values using Upper Range COVs.

\begin{tabular}{|c|c|c|c|c|c|}
\hline $\begin{array}{l}\text { Sliding } \\
\text { PUP Target }\end{array}$ & Sliding $\beta$ Target & Sliding $R_{S F}$ & Sliding $L_{S F}$ & $P^{\prime} P_{\text {System }}$ & $\beta$ system \\
\hline * & * & 1.000 & * & 0.027759 & 1.91 \\
\hline 0.158655 & 1.00 & 0.836 & 1.166 & 0.175754 & 0.93 \\
\hline 0.066807 & 1.50 & 0.909 & 1.269 & 0.074580 & 1.44 \\
\hline 0.022750 & 2.00 & 1.009 & 1.407 & 0.025468 & 1.95 \\
\hline 0.006210 & 2.50 & 1.150 & 1.603 & 0.008208 & 2.40 \\
\hline 0.001350 & 3.00 & 1.356 & 1.891 & 0.003716 & 2.68 \\
\hline 0.000233 & 3.50 & 1.734 & 2.418 & 0.001969 & 2.88 \\
\hline 0.000159 & 3.60 & 1.802 & 2.512 & 0.001856 & 2.90 \\
\hline 0.000108 & 3.70 & 1.924 & 2.683 & 0.001774 & 2.92 \\
\hline 0.000072 & 3.80 & 2.053 & 2.863 & 0.001752 & 2.92 \\
\hline 0.000048 & 3.90 & 2.143 & 2.988 & 0.001747 & 2.92 \\
\hline 0.000032 & 4.00 & 2.413 & 3.365 & 0.001715 & 2.93 \\
\hline
\end{tabular}

*This value is not a result of assigning the Sliding $\beta_{\text {Target, }}$ but a reflection of running the integration at parity, where $R_{S F}=1 . O$.

Figure 5 -19 shows the relationship between the Sliding $\beta_{\text {Target }}$ and the result $\beta$ System using the $R_{S F}$ for that $\beta_{\text {Target. }}$ As the Sliding $\beta_{\text {Target }}$ increases above a value of 2.5, there is a noticeable divergence between $\beta$ System and Sliding $\beta_{\text {Target. }}$. The dashed red line shows the expected relationship if the Sliding load factors were the driving condition for the system. This would 
be the case if there were only one dominant limit state (i.e., Sliding) and no system response effects contributed by the other four limit states.

Figure 5-19. Comparison of system $\beta$ values computed using increasing Sliding $R_{S F}$ to the Dominant Sliding Limit State $\beta$ Target values.

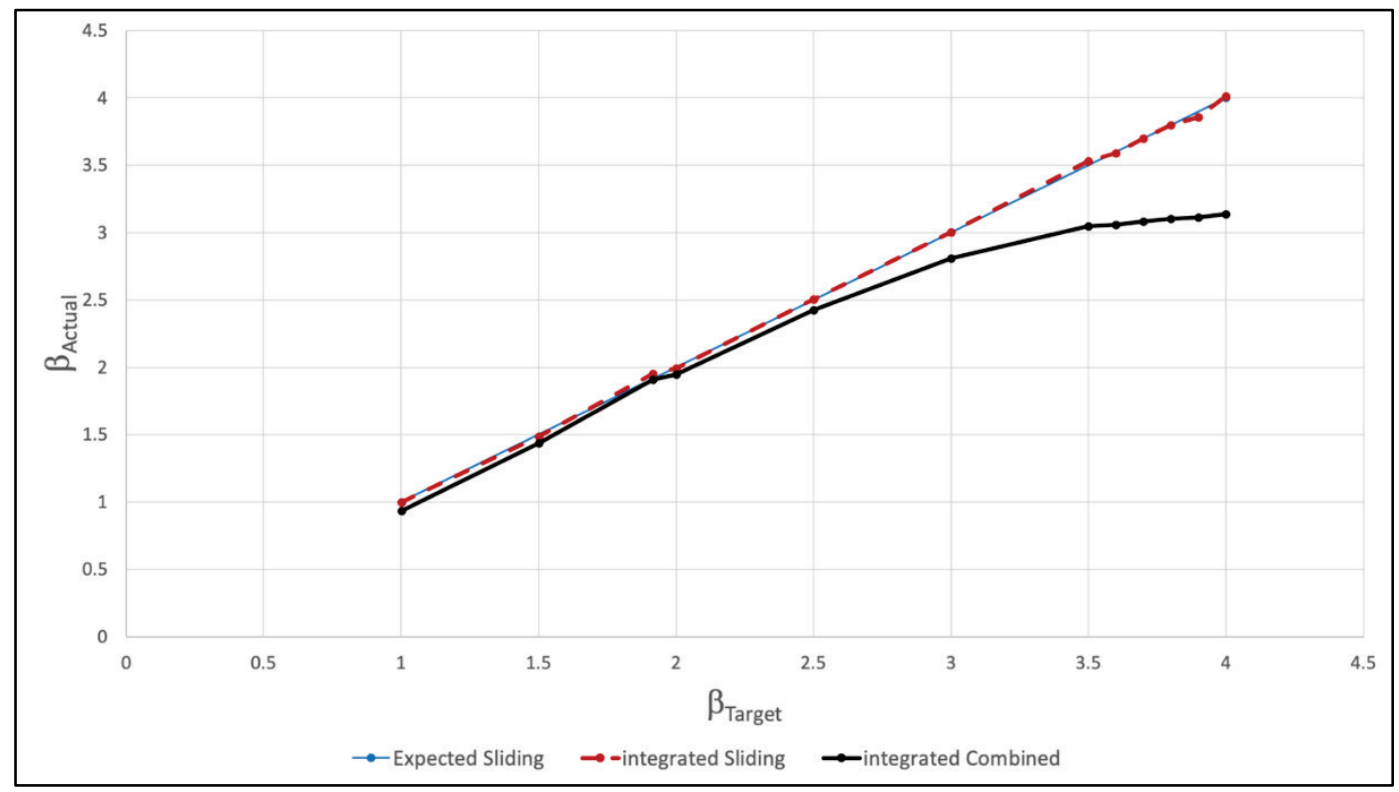

Table 5-7 shows this relationship as ratios of the System values (PUP and $\beta$ ) with respect to the Sliding Target values. The Pupsystem vs. PUPTarget values for a $\beta$ Target greater than 2.5 begin to increase showing further disparity. The ratio values for $\beta$ Target greater than or equal to 3.0 are greater than 2.7. This PUP System to $P U P_{\text {Target }}$ divergence is more noticeable than the relationship divergence of $\beta_{\text {System }}$ to $\beta_{\text {Target }}$. 
Table 5-7. Examining the ratios of PUPand $\beta$ for Sliding limit state target and integrated 5-D system results.

\begin{tabular}{|c|c|c|c|c|c|}
\hline $\begin{array}{l}\text { Sliding } \\
\text { PUPTarget }\end{array}$ & $\begin{array}{l}\text { Sliding } \\
\boldsymbol{\beta}_{\text {Target }}\end{array}$ & PUP $_{\text {System }}$ & $\beta_{\text {System }}$ & $P U P_{\text {System }} / P P_{\text {Target }}$ & $\beta_{\text {System }} / \beta_{\text {Target }}$ \\
\hline 0.158655 & 1.00 & 0.175754 & 0.93 & 1.108 & 0.932 \\
\hline 0.066807 & 1.50 & 0.074580 & 1.44 & 1.116 & 0.962 \\
\hline 0.022750 & 2.00 & 0.025468 & 1.95 & 1.119 & 0.976 \\
\hline 0.006210 & 2.50 & 0.008208 & 2.40 & 1.322 & 0.960 \\
\hline 0.001350 & 3.00 & 0.003716 & 2.68 & 2.753 & 0.892 \\
\hline 0.000233 & 3.50 & 0.001969 & 2.88 & 8.464 & 0.824 \\
\hline 0.000159 & 3.60 & 0.001856 & 2.90 & 11.665 & 0.806 \\
\hline 0.000108 & 3.70 & 0.001774 & 2.92 & 16.456 & 0.788 \\
\hline 0.000072 & 3.80 & 0.001752 & 2.92 & 24.216 & 0.768 \\
\hline 0.000048 & 3.90 & 0.001747 & 2.92 & 36.323 & 0.749 \\
\hline 0.000032 & 4.00 & 0.001715 & 2.93 & 54.150 & 0.732 \\
\hline
\end{tabular}

The divergence of PUP System from PUPSliding in Table 5-6 (and in terms of $\beta$, Figure 5-15) may be explained by the fact that the correlation between the load and resistance is not taken into account in the 5 -D adaptive hyperspherical Gaussian probability numerical integration solution method. This proportioned response is discussed in Section 4.6. Briefly stated, for the three-soil wedge solution method, the load mean increases as the resistance mean is increased by a scale factor because of design changes. To determine the estimated correlated rate of change between the load and resistance distribution means as the design changes, at least two designs need to be analyzed with the same load conditions so that the rate of change of the mean values can be measured for the two distributions. However, load and resistance distributions can only be collected for a single limit state in the three-wedge solution using Monte Carlo methods and storing correlated values.

The results discussed in this chapter demonstrate that using a single, dominant limit state to determine the system load and resistance factors does not consider the complexity of multiple, overlapping limit states which can have a cumulative effect. The devised hybrid adaptive hyperspherical Gaussian probability numerical integration model results for the 5-D reliability problem of this chapter demonstrate that this sort of analysis can be used to improve Load and Resistance Factors for LRFD procedures. 
Considering the observations made by Allen (2005) and Fenton et al. (2016) regarding non-redundant foundations, the part of the Figure 5-16 curve of most interest is in the $\beta$ value range of 3.1 to 3.7, with a typical $\beta$ value of around 3.5 for an American Association of State Highway and Transportation Officials (AASHTO) and Canadian bridge foundation. Allen (2005) states that the landmark Barker et al. (1991) reliability study of bridges (75-year design life) and the various types of foundation systems suggests a target $\beta$ value of 3.5 for non-redundant systems such as footings. ${ }^{1}$ Based upon Fenton et al. (2016), the equivalent reliability-based target $\beta$ values of Canadian bridge foundation design is in the range of 3.1 to 3.7, depending upon the consequence of its failure. In discussing the reliability formulation background to the Canadian Highway Bridge Design Code (CHBDC) (CSA 2014) for foundations or geotechnical systems design, Fenton et al. (2016) provide their range for the target maximum lifetime (75-year) failure probabilities, $p_{m}$, and equivalent reliability indices for the foundations ULS depending upon the consequence level (Table 5-8). Three consequence levels were stipulated: high, typical, and low. A foundation that supports a lifeline bridge or hospital is an example of a high consequence level should a foundation failure occur. Conversely, if the foundation supports a storage warehouse that is rarely visited, the failure consequences are slight, and the consequence level is judged to be low.

\footnotetext{
${ }^{1}$ An example of a redundant foundation system is a pile group consisting of five or more piles. Allen (2005) states that Barker et al. (1991) conclude that a target $\beta$ value of 2 to 2.5 for a redundant system is appropriate. Zhang et al. (2001) and Paikowsky et al. (2004) have also concluded that for pile groups, a $\beta$ of 2.3 is reasonable to target when considering the resistance needed for a single pile located within a group of significant size. Their reliability index value falls midway in the Barker et al. range recommendation.
} 
Table 5-8. Targeted theoretical maximum lifetime (75-year) failure probabilities, $p_{m}$, and equivalent reliability indices, $\beta$, for the ULS in the 2014 CHBDC for foundations or geotechnical systems (after Fenton et al. 2016).

\begin{tabular}{|l|l|l|}
\hline $\begin{array}{l}\text { Consequence } \\
\text { Level }\end{array}$ & $p_{\mathrm{m}}$ & $\beta$ \\
\hline High & $1 / 10,000$ & 3.7 \\
\hline Typical & $1 / 5,000$ & 3.5 \\
\hline Low & $1 / 1,000$ & 3.1 \\
\hline
\end{tabular}

Subsection 4.9.2 discusses the adjustment of the Allen (2005) and Fenton et al. (2016) reliability index $\beta$ values from a 75-year bridge design life to a 100year project life used for USACE hydraulic structures. Recall Allen's (2005) reported target reliability index $\beta$ value of 3.5 for footings and other nonredundant (AASHTO 2012) systems becomes 3.46 for projects with a 100-year design life based on the Ebeling and White (2019) calculations and their Table 2-8 results. This is the same $\beta$ equal 3.5 design value used for the Canadian Bridge Code (CSA 2014) in the case of bridge foundations for an Ultimate Limit State with a typical consequence level according to Fenton et al. (2016). Fenton et al.'s (2016) $\beta$ of 3.7 for the Ultimate Limit State with a high consequence level becomes 3.65 for a 100-year project life.

Concluding observations: The observations made for the T-Wall retaining wall reliability problem discussed in this chapter consider the nonlinear relationships that have been observed throughout the numerical research involved in this project. It also reveals that correlation is to be expected in a three-wedge analysis of geotechnical retaining structures when all three wedges (i.e., Driving, Structural, and Resisting) are incorporated in the engineering model of the reliability analysis. These are important aspects of the Reliability analysis, impacting computed results. 


\section{Summary, Conclusions, and Research Recommendations}

\subsection{Summary}

This report focuses on a Load and Resistance Factor Design (LRFD) for complete, reinforced concrete hydraulic structures. Complete hydraulic structural analysis involves the geotechnical and structural limit states, as well as the soil structure interactions (SSI) induced by loading. A total of five limit states are incorporated in the engineering model's reliability analysis of a hydraulic structure discussed in this report. These are

- global sliding failure of the structural system in its entirety

- bearing failure of the foundation to the structural system in its entirety

- overturning or rotational failure of the structural system about its foundation in its entirety

- shear failure of individual structural members

- flexural hinging of individual structural members.

The geotechnical reliability research results of Fan et al. (2014) demonstrate that all five limit states need to be considered concurrently and not independently, for all variables in the reliability analysis in order to compute an accurate $P U P$ value, and ultimately, accurate reliability results for use in the computation of load factors.

Combined LRFD, involving geotechnical as well as structural limit states, is an area that has not been addressed by the USACE. While research has been conducted for structural LRFD where the materials are more uniform, geotechnical research has been complicated by spatial variability of engineering material properties for the soil(s). The companion Phase 1 Study (Ebeling and White 2019) documents the initial investigation into reliability methodologies considered for application to the LRFD analysis of geotechnical regimes for reinforced concrete hydraulic structures. It also described initial development of numerical procedures with enough precision for a complete LRFD analysis of the reinforced concrete, hydraulic structures. This Phase 2 Study continues the development of these analytical methods and new numerical procedures and applies them to a reinforced concrete T-Wall example. The result is a Reliability-based LRFD analysis of an example T-Wall possessing multiple geotechnical and structural limit states. 
In that vein, this technical report has focused primarily on building engineering models that capture soil-structure interaction and are readily adaptable to reliability analysis of a USACE reinforced concrete T-Wall buttressed by and retaining earth. This holds true with respect to the model for first engineering limit state discussed, which centers on the sliding stability analysis of a T-Wall. A T-Wall example structure was chosen to lay out the engineering and reliability analysis capabilities needed because it possesses the attributes of all three wedges in its geotechnical numerical model: a driving wedge, a structural wedge, and a resisting wedge. The engineering and reliability principles proposed for use are broad enough to be used for overturning and bearing failure limit states as well. The engineering model also contains the shear and flexural structural limit states of the reinforced concrete stem. In summary, this Phase 2 Study numerical T-Wall model example possesses all five geotechnical and structural limit states.

Modeling variability in soils is complicated by the fact that even "homogeneous" soil engineering material properties change with relative distance. With enough difference in distance, the soil strength properties can be completely unrelated to the distribution at the original location. This led to the development of three different models being proposed in the Phase 1 Study (Ebeling and White 2019). The first devised method uses an earth pressure coefficient method to compute forces acting against the structural wedge. The other two devised methods use the slip plane as a basis for generating homogeneous material regions. One method created numerous small sub-wedge features of scale of fluctuation width along the slip plane assigned with material properties from the original soil property distributions before applying the Variance Reduction Factor. The second method described in Subsection 4.4.2 of Ebeling and White (2019) is used in this report. This second method computes the slip plane length for each of the three wedges and computes a Variance Reduction Factor based on the slip plane length and the scale of fluctuation of the material properties for each of the three wedges. This variance reduction factor is used to establish a new distribution for each wedge convoluting the probabilities over the longer slip plane run, accounting for spatial correlation along the slip plane. Each of the three wedges possesses a Variance Reduction Factor based on the length of the slip plane and the Scale of Fluctuation for that soil region. The new soil property distribution has the same means but the $\mathrm{COV}$ is reduced from the COV Point value to a $C O V_{\text {Spatial }}$ value based on the slip plane length and its orientation. 
These engineering models can be used in a reliability analysis to determine the Probability of Unsatisfactory Performance (PUP) and reliability index $(\beta)$ for the geotechnical/structural system. A reliability analysis can compute a value for PUP in a few different ways. Early methods researched and discussed in the Phase 1 Study (Ebeling and White 2019) dealt with the Advanced Second Moment (ASM) method and Monte Carlo simulation techniques. The ASM method by itself only works for a single hyperplanar limit state for estimating PUP, which does not serve the geotechnical/structural systems in this report because the PUP system value would be underestimated because the ASM method does not give results for multiple overlapping limit states. However, the ASM method determines the closest approach of the limit state response surface, giving a good $\beta$ value and directional cosines $(\alpha)$ that define a design point on the response surface.

Monte Carlo simulation techniques can achieve greater accuracy than the ASM method given enough simulation samples. Monte Carlo techniques return a PUP value but do not give an accurate $\beta$ or $\alpha$ value unless an ASM search for a design point has been performed. It is also possible, given random selection of samples, that not every limit states contribution will be included in the PUP calculation.

Appendix $\mathrm{C}$ begins by introducing three methods for computing load and resistance factors. The first method uses engineering Reliability standards. The second method uses the Section C.2 numerical method that calculates a Load-to-Resistance ratio which, combined with a prescribed Resistance Factor, the Section C.3 method computes the corresponding Load Factor. Finally, the third method uses the Section C.5 numerical procedure that calculates a $k$ value (for the $\max \beta$ ) that can be applied in Equations C.40 and C.41.

Appendix $\mathrm{C}$ also introduces techniques for computing $\beta$ given load and resistance factors either directly or indirectly depending on variable distributions. The Section C.4 numerical procedure attempts to iterate to find a maximum $\beta$ given resistance and load distribution descriptions using Rackwitz and Fiessler $(1976,1978)$ Gaussian Normal curve approximates. The Section C.5 numerical procedure computes the maximum $\beta$ and its associated $k$ value. Given an arbitrary $k$ value and substituting it into equation C. 42 will return a maximum $\beta$ value, also. Section C.6 attempts to indirectly calculate the $\beta$ value for a non-normal distribution by summing 
fractional Gaussian curves to fit the non-normal distribution with low error. The fractional curve properties are used in equation C. 45 to determine the $\beta$ value for a non-normal distribution.

The authors also conducted a Monte-Carlo simulation with a large number of samples on the resistance and load curves and calculated the $(R-L)$ curve with binned histograms. The PUP value was then calculated by integrating probabilities for the resulting distribution up to the safety margin $(R-L)=0$ response surface and an inverse normal distribution cumulative distribution function (CDF) calculation was used to get a transformed Gaussian Normal space $\beta$ value estimate.

These Appendix C methods work for single limit states with well-defined traditional distributions for resistance and load. Additional investigation reveals that structures with geotechnical features do not have these welldefined, uncorrelated distributions for load and resistance.

In this Phase 2 study, a numerical model for a T-wall was created (Chapter 3) and analyzed for all five limit states, geotechnical and structural. Limit state solutions were performed for individual limit states using ASM and Monte Carlo simulation methods (Chapter 4). These methods were capable of determining probability density function (PDF) distributions of loading and resistance from variable soil properties and application of numerical procedures. These loads were computationally non-linear according to analysis of the geotechnical wedges based on soil properties. The establishment of these non-linear load and resistance PDF distributions led to the development of methods for determining load and resistance factors by numerical calculation of a scale factor for the resistance PDF distribution such that samples from the scaled resistance and unscaled load would yield the target $P U P$ for the system (Section 4.2).

Discovery that these soil-based PDF distributions, load and resistance, were not orthogonal but correlated as design changes were made to the structure required extending the previous load and resistance factor calculation procedure (Section 4.2) to include a proportionality value based on the rate of change of load and resistance. In this case, scaling the resistance PDF distribution had a correlated proportional scaling effect on the load PDF distribution. This procedure is discussed in Section 4.6. 
Both of these procedures (Sections 4.2 and 4.6) produce a ratio of factored load to factored resistance for a target PUP value. Section 4.7 provides a means to calculate either the load or resistance factor, when the other factor is fixed. With the ratio and a given resistance factor, the load factor can be computed and vice versa. Given guidance for resistance factors, which is a usual occurrence in USACE guidance, a load factor can be computed to establish an agreed upon PUP.

Section 4.8 took the lessons learned and the procedures developed in the previous sections of Chapter 4, which were applied to the Example Wall with Extreme geometry (Figure 4-3b) and processed the wall with Upper Range coefficients of variation (COVs) for the effective angles of internal friction in each of the soil regions. This variability establishes the true uncertainty for the Extreme condition. Figure 4-9 shows the relationship between computed Load Factors and the equivalent $\beta$ Target for the Example T-Wall with Upper Range material property COVs.

A numerical model for a T-Wall was created and analyzed using all five geotechnical and structural limit states. Limit state solutions were performed for individual limit states using ASM and Monte Carlo simulation methods. These methods were capable of determining distributions of loading and resistance based upon variable soil properties. Also, an adaptive hyperspherical Gaussian probability numerical

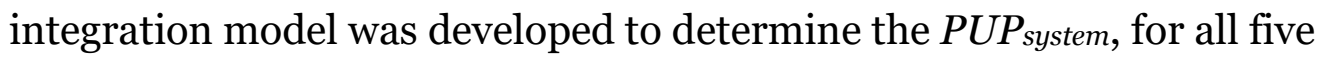
limit states, given the engineering model of a soil-founded T-Wall. This devised adaptive hyperspherical Gaussian probability numerical integration model and its hybridization are described in Subsections 5.3.2.2 and 5.3.2.3. The method had to be further extended to deal with individual variables that could have different distributions for different limit states. This model was applied to a three-geotechnical limit state scheme with three geotechnical variables in Section 5.5. This analysis results in computation-based diagrams that illustrate the Monte Carlo simulation and adaptive hyperspherical Gaussian probability numerical integration procedures and compares their performance. Both the resulting PUPsystem and the plots from this analysis reveal that the Sliding limit state is the primary limit state. The other two Geotechnical limit states of Bearing and Base Area in Compression possess extremely low values for the probability of unsatisfactory performance. 
This Section 5.5 analysis is extended in Section 5.6 where all five limit states, geotechnical and structural, are combined into a single PUP system value. The addition of the two Structural limit states introduced two new distributed variables. These two new variables did not have an effect on the geotechnical limit states. One of the new distributed variables gave the resistance of the stem to the shear limit state, and the other gave the resistance of the stem to the flexural moment limit state. The addition of these two limit states and their variables made a difference in the $P U P_{\text {system }}$ calculation. While the Geotechnical Sliding limit state was still the primary source of probability of failure, the Structural Stem Shear limit state had a significant contribution to the overall PUP system, with an estimated Gaussian $\beta$ value in the range of 3.1. A surprising result for the authors of this report was that although the limit states had a large overlap of probabilities, the PUP system appeared additive of the two limit states. This would be expected for parallel limit states. But these two limit states are not parallel. This observation is explained by realizing the overlap region has such an insignificant probability that doubling its effect was still insignificant.

\subsection{Conclusions}

In conclusion, given two full distribution descriptions for load and resistance the simulation method (with enough samples) and the Section C. 5 procedural method can determine an accurate PUP value either directly or through a normal distribution $\mathrm{CDF}$ calculation from a calculated $\beta$. Based on extensive series of trial and error computations, the Section C.6 method was found to be an interesting mathematical exercise but has too many variables and needs restraint. Results from Reliability analyses summarized at the end of Section C.2 showed that the $\beta$ calculation method for non-normal distributions requires small steps using normal distributions mapped to the non-normal distribution at a given design point (Rackwitz and Fiessler 1976, 1978), which can have problems with accuracy in the tails region.

The Section C. 2 procedural method is the only method that attempts to numerically establish load and resistance factors that will scale the positions of existing distributions so that the safety margin $(R-L)$ response surface will have a $P U P$ value that corresponds to a normal distribution $\beta_{\text {target }} \mathrm{CDF}$ value. Unfortunately, this procedure is attempting to find a value in the tail of the distribution and therefore has accuracy problems that are revealed in the results tables in this section of the appendix. 
The individual limit state procedure for computing Load and Resistance Distributions from material properties and determining load factors (Section 4.2) provides a method for computing a ratio of load and resistance factors for an individual Limit State. This method works with orthogonal distribution sets for load and resistance as computed from material properties. This method relies on the fact that material properties follow the Central Limit Theorem of probability in large numbers (Henk 2004) and therefore have typical bell-shaped distributions and that the load and resistance factors may have non-linear equations for their distribution formation from those material properties.

The devised Section 4.2 method was extended to the more useful proportioned load individual Limit State procedure for computing Load and Resistance distributions from material properties and determining load factors (Section 4.6). This procedure assumes some level of correlation expressed as a proportion between the load and resistance distribution as the structural design changes, as evidenced in Figures 4-2 and 4-3. A scale factor is computed from the rates of change of load and resistance, and that scale is applied for the estimation of the ratio of load and resistance scale factors. This numerical procedure improves the speed at which the computations are made, an important capability for conducting simulations of the T-Wall in a Reliability analysis.

Section 4.7 reveals how the proportion of load factor and resistance factor can be used to determine one factor if the other is specified. This is helpful, since resistance factors are routinely specified in USACE guidance (e.g., EM 1110-2-2104 [HQUSACE 2016]).

Monte Carlo and adaptive hyperspherical Gaussian probability numerical integration of PUP system for the three geotechnical limit states alone revealed that Sliding was the most probable geotechnical limit state to fail for the Chapter $2 \mathrm{~T}$-Wall system example problem. The adaptive hyperspherical Gaussian probability numerical integration provides a level of accuracy for approximately the same level of effort (e.g., number of model runs) as the Monte Carlo methods. Plots from these methods revealed the non-planarity of the resulting response surfaces, contrary to the estimates given by the ASM solution for the single limit states.

Combining geotechnical with structural limit states, Monte Carlo and adaptive hyperspherical Gaussian probability numerical integration of the 
$P U P_{\text {system }}$ was performed again to find out if the new limit states would have a noticeable effect. The Stem Shear Limit State provided a significant contribution to $P U P_{\text {system. }}$. Finding load and resistance factors that reduce the $P U P_{\text {system }}$ will be determined by the rate of change of these limit state response surfaces with respect to the factors.

Table 3-1 in EM 1110-2-2104 (HQUSACE 2016) guidance for the Extreme load case for lateral earth pressures provides values for a Resistance Factor of 0.5 and a Load Factor of 1.4. A Load Factor value of 1.4 corresponds to a Reliability Index value of approximately 3.8 by the data given in Figure 4-9 for Upper Range COV values for effective angles of internal friction. The $\beta$ value of 3.8 for a USACE navigation structure falls approximately midway between the Ellingwood reliability index recommendation for normal and critical structures. This computed $\beta$ value of 3.8 exceeds the historical allowable stress design (ASD) foundation design $\beta$ experience (Allen 2005) as well as the guidance for the 2014 Canadian Bridge Design Code as described by Fenton et al. (2016). Thus, the EM 1110-2-2104 (HQUSACE 2016) guidance LRFD values for load and resistance factors for the driving and resisting earth pressure loads applied to the Structural Wedge in the sliding limit state are deemed slightly conservative but not excessive by the authors of this report when a $\beta$ of 3.8 value is compared to the target $\beta$ values given by Allen (2005) and Fenton et al. (2016). This assessment may be improved by making a Reliability assessment of other earth-retaining USACE hydraulic structures following the procedure of analysis outlined in this technical report.

The key conclusions are as follows:

- Research of previously devised methods for the determination of PUP values reveal that traditional, Structural Engineering-based Reliability methods, described in Appendix C, have limitations that prohibit their use for multi-Limit State problems with correlation and complicated distributions, which is the case with geotechnical structures.

- A T-Wall Analysis Example problem was developed with the geometry determined for the usual load conditions of the Normal Load Case and solved using EM 1110-2-2100 (HQUSACE 2005) stability criteria as well as procedures for calculating the geotechnical forces that affected each limit state. It is important to recall that not all three Geotechnical limit states dictated the T-Wall geometry to satisfy minimum design 
guidance. It was the Sliding Limit State that dominated the geometry requirements.

- Methods for determining distributions of Load and Resistance forces for different Limit States given the geotechnical problem of an Example TWall using histogram data of Monte Carlo simulations based on soilstrength properties were developed. The development of these methods revealed that the Load and Resistance distributions have correlations for the geotechnical Wedge-based solution.

- Procedures for devising Load and Resistance factors from the collected, correlated Monte Carlo simulation values for individual limit states were determined, assuming that the Load and Resistance distributions were uncorrelated (Section 4.2). It was discovered that this assumption was not true.

- Because the Load and Resistance distributions were correlated, the Section 4.2 procedure was extended to allow for correlation (Section 4.6). Accounting for the effects of correlated Load and Resistance functions is essential for the determination of accurate Load and Resistance Factors.

- The Usual Load Case (Table 3-1 of EM-1110-2-2104 [HQUSACE 2016]) design of the T-Wall uses USACE criteria (EM-1110-2-2502 [HQUSACE 1989]) with a Factor of Safety (FS) against Sliding of 1.5 (EM-1110-22100 [HQUSACE 2005]). Table 4-1 reveals that the designed T-Wall structure has a PUP value of 0.00025 using the anticipated Mid-Range COV values for effective angle of internal friction (Table 2-1), which equates to a $\beta$ of 3.48 (Table 4-1) for the dominant limit state of sliding. This value is consistent with first-generation AASHTO guidance (Allen 2005) value of 3.46 and Canadian Bridge Code (CSA 2014) for an Ultimate Limit State (ULS) with a typical consequence level but is slightly lower than the extrapolation to 100-year design life value of 3.65 for the 2014 Canadian bridge code (Fenton et al. 2016) ULS with a high consequence level. (Refer to Subsection 4.9.2 for a discussion of the conversion from a 75-year bridge design life to a 100-year project life used for USACE hydraulic structures.) It is observed that the ASD normal load case design using USACE criteria appears to be generally consistent with Allen (2005) and Fenton et al. (2016) foundation reliability targets.

- The Extreme Load Case uses the same T-Wall that has been dewatered. The values for the Extreme Load Case with anticipated Mid-Range COV values for effective angle of internal friction (Table 2-1) gives a PUP of 0.00175 and $\beta$ of 2.92 (Table 4-2) for the dominant sliding limit state, 
which are noticeably lower than the Usual load case values. This validates the expectation that the reliability of the foundation is reduced as the severity of loading increases.

- Most importantly, Table 3-1 of EM 1110-2-2104 (HQUSACE 2016) recommends for lateral earth pressures Load and Resistance Factors for the Extreme Load Case to be 1.4 and 0.5, respectively. Figure 4-9 results, using the procedure of Subsection 4.6 applied to the dominant limit state of sliding, reveals that for a Load Factor of 1.4, determined using a Resistance Factor of 0.5, gives a $\beta$ value of 3.8 for Upper Range soil strength COV values. The calculated value for $\beta$ of 3.8 is deemed adequate for the critical Sliding Geotechnical Limit State with a 100year project life based on the LRFD information contained within Allen (2005), and Fenton et al. (2016) adjusted to a 100-year project life (Subsection 4.9.2) but falls between the Ellingwood reliability indices recommended values for non-critical structures $(\beta=3.5)$ and critical structures $(\beta=4.0)$ with a single load path.

- Using Figure 4-9 results for the dominant limit state of sliding, a load factor of 1.2 is sufficient to achieve a target $\beta$ equal to 3.5 , which is required according to Ellingwood for a noncritical structure with a single load path, given a specified resistance factor of 0.5 according to EM 1110-2-2104 (HQUSACE 2016) guidance. This Load Factor value is $14 \%$ lower than the reported EM 1110-2-2104 load factor of 1.4 with the same specified resistance factor.

- A method for the determination of overall PUP of the Example T-Wall problem, considering all of the Limit States concurrently, was implemented and was compared to the individual Limit State PUP values. This is an essential requirement for a complete and accurate system $P U P$ value. Computation of $P U P_{\text {System }}$ reveals that the PUPSystem value is greater than any individual Limit State PUP value.

- To generalize, when PUPSystem is greater than PUP for the dominant limit state evaluated on its own, the load factor from the individual limit state assessment may need to be increased. The PUP $P_{\text {System }}$ needs to be computed as the individual limit state load factor is being created. Note that the rate of change for PUP as each resistance is scaled (Figure 5-16) can cause the dominant Limit State to change. 


\section{References}

AASHTO (American Association of State Highway and Transportation Officials). 2010. AASHTO LRFD Bridge Design Specifications, $5^{\text {th }}$ edition, Parts I and II. Published by American Association of State Highway and Transportation Officials, Washington, D.C.

AASHTO. 2012. AASHTO LRFD Bridge Design Specifications, American Association of State Highway and Transportation Officials, 6th Edition. Washington DC.

ACI (American Concrete Institute) Committee 318. 2014. Building Code Requirements for Structural Concrete (ACI 318-14) and the Commentary (ACI 318R-14). Farmington Hills, MI: American Concrete Institute.

ACI Committee 340. 1978. Design Handbook in Accordance with the Strength Design Method of ACI 318-77, Volume 2, Columns. ACI Publication SP-17A(78). Detroit, MI: American Concrete Institute.

ACI. 2008. Building Code Requirements for Structural Concrete and Commentary. Farmington Hills, MI: American Concrete Institute.

Allen, T. M. 2005. Development of Geotechnical Resistance Factors and Downdrag Load Factors for LRFD Foundation Strength Limit State Design, Reference Manual. U.S. Department of Transportation, Federal Highway Administration, Publication No. FHWA-NHI-05-052. Washington DC: National Highway Institute.

Ang, A. H-S., and W. H. Tang. 1984. Probability Concepts in Engineering Planning and Design: Decision, Risk and Reliability, volume II.

Ang, A. H-S., and W. H. Tang. 2007. Probability Concepts in Engineering, Emphasis on Applications to Civil and Environmental Engineering, Volume I, 2nd Edition. New York: John Wiley \& Sons.

ASCE (American Society of Civil Engineers) Standard. 2010. Minimum Design Loads for Buildings and Other Structures. ASCE/SEI 7-10.

Babu, G. L. S. 2014. Reliability Engineering. National Programme on Technology Enhance Learning (NPTEL). DOER - Directory of Open Educational Resources. http://doer.col.org/handle/123456789/3939.

Babu, G. L. S., and S. M. Dasaka. 2007. "The Effect of Spatial Correlation of Cone Tip Resistance on the Bearing Capacity of Shallow Foundations." Geotech Geol Eng. Springer Science+Business Media. https://link.springer.com/article/10.1007/s10706007-9145-4.

Baker, J., and E. Calle. 2006. JCSS Probabilistic Model Code. Section 3.7: Soil Properties. Joint Committee on Structural Safety. Updated version Aug. 2006, Zurich, Switzerland.

Barker, R. M., J. M. Duncan, K. B. Rojiani, P. S. K. Ooi, C. K. Tan, and S. G. Kim. 1991. Manuals for the Design of Bridge Foundations. NCHRP Report 343, TRB. Washington, DC: National Research Council. 
Bathurst, R. J., T. M. Allen, and A. S. Nowak. 2008. "Calibration Concepts for Load and Resistance Factor Design (LRFD) of Reinforced Soil Walls.” Canadian Geotechnical Journal 45: 1377-1392.

Benjamin, J. R., and C. A. Cornell. 1970. Probability, Statistics, and Decision for Civil Engineers. New York: John Wiley \& Sons.

Chen, W. F., and X.L. Liu. 1990. Limit Analysis in Soil Mechanics. Developments in Geotechnical Engineering 52. New York: Elsevier Science Publishers, Amsterdam.

CSN (Canadian Standards Association). 2014. Canadian Highway Bridge Design Code. CAN/CSA-S6-14. Mississauga, Ont.

Dawkins, W. P. 1984. User's Guide: Computer Program for Soil-Structure Interaction Analysis of Axially Loaded Piles (CAXPILE). Instruction Report K-84-4. Vicksburg, MS: U.S. Army Engineer Waterways Experiment Station.

Department of the Navy. 1982. Foundations and Earth Structures. NAVFAC Design Manual DM 7.2. Alexandria, VA: Naval Facilities Engineering Command.

Ebeling, R. M. 1989. Methods of Evaluating the Stability and Safety of Gravity Earth Retaining Structures Founded on Rock. Thesis for Doctorate of Philosophy in Civil Engineering, Virginia Polytechnic Institute and State University, Blacksburg, VA.

Ebeling, R. M., and E. E. Morrison. 1992. The Seismic Design of Waterfront Retaining Structures. Technical Report ITL-92-11. Vicksburg, MS: U.S. Army Engineer Waterways Experiment Station.

Ebeling, R. M., and B. C. White. 2019. Load Factors for Earth Retaining, Reinforced Concrete Hydraulic Structures Based on a Reliability Index $(\beta)$ Derived from the Probability of Unsatisfactory Performance; Phase 1 Study. ERDC/ITL TR-19-5. Vicksburg, MS: U.S. Army Engineer Research and Development Center.

Ebeling, R. M., B. C. White, and M. T. Fong. 2013. Simulation and Advanced Second Moment Reliability Analyses of Pile Groups using CPGA-R; Formulation, User's Guide, and Example Problems. ERDC/ITL TR-13-2. Vicksburg, MS: U.S. Army Engineer Research and Development Center.

El-Ramly, H., N. R. Morgenstern, and D. Cruden. 2002a. "Probabilistic Slope Stability Analysis for Practice.” Canadian Geotechnical Journal 39: 665-683.

El-Ramly, H., N. R. Morgenstern, and D. Cruden. 2002b. "Probabilistic Stability Analysis of Lodalen Slide. Proc., 55th Canadian Geotechnical Conf., Niagara Falls, 10531060.

Fan, H., Q. Huand, and R. Liang. 2014. "Reliability Analysis of Piles in Spatially Varying Soils Considering Multiple Failure Modes." Computers and Geotechnics 57: 97104. doi:10.1016/j.compgeo.2014.01.009.

Fenton, G. A., F. Naghibi, D. Dundas, R. J. Bathurst, and D. V. Griffiths. 2016. "ReliabilityBased Geotechnical Design in 2014 Canadian Highway Bridge Design Code. NRC Research Press, Canadian Geotechnical Journal 53: 236-251. 
Ghasemi, S. H. 2015. Target Reliability Analysis for Structures. Ph.D. Dissertation for the Degree of Doctoral of Philosophy, Auburn University, Auburn, AL.

Hasofer, A. M., and N. C. Lind. 1974. "Exact and Invariant Second Moment Code Format." Journal of the Eng. Mech. Division, ASCE 100(EM1): 111-121.

Henk, Tijms. 2004. Understanding Probability: Chance Rules in Everyday Life. Cambridge: Cambridge University Press.

HQUSACE (Headquarters, U.S. Army Corps of Engineers). 1989. Retaining and Flood Walls. EM 1110-2-2502. Washington, DC.

HQUSACE. 2005. Stability Analysis of Concrete Structures. EM 1110-2-2100. Washington, DC.

HQUSACE. 2016. Strength Design for Reinforced Concrete Hydraulic Structures. EM 1110-2-2104. Washington, DC.

Meyerhof, G. G. 1963. "Some Recent Research on the Bearing Capacity of Foundations." Canadian Geotechnical Journal 1(1): 16-26

Nowak, A. S. 1999. Calibration of LRFD Bridge Design Code. National Cooperative Research Program Report 368. Washington D.C.: Transportation Research Board, National Research Council, National Academy Press.

Nowak, A. S., and N. C. Lind. 1979. "Practical Bridge Code Design." ASCE Journal of the Structural Division 105(ST12): 2497-2510.

Nowak, A. S., and K. R. Collins. 2000. Reliability of Structures. Boston: McGraw-Hill.

Nowak, A. S., and K. R. Collins. 2013. Reliability of Structures, 2nd ed. Boca Raton, FL: CRC Press, Taylor and Francis Group.

Pace, M. E. 1994. User's Guide to CTWALL - A Microcomputer Program for the Analysis of Retaining and Flood Walls. Instruction Report ITL-94-7. Vicksburg, MS: U.S. Army Engineer Waterways Experiment Station.

Paikowsky, S. G. (contributions from B. Birgisson, M. McVay, T. Nguyen, C. Kao, G. Baecher, B. Ayyub, K. Stenersen, K. O’Malley, L. Chernauskas and M. O’Neil). 2004. Load and Resistance Factor Design (LRFD) for Deep Foundations. NCHRP (Final) Report 507. Washington, DC: Transportation Research Board.।

Park, C., N. H. Kim, and R. T. Haftka. 2015. The Effect of Ignoring Dependence between Failure Modes on Evaluating System Reliability, Structural and Multidisciplinary Optimization.

Pearson, K. 1895. "Notes on Regression and Inheritance in the Case of Two Parents." Proceedings of the Royal Society of London,

Peck, R. B., W. E. Hanson, and T. H. Thornburn. 1974. Foundation Engineering, 2nd edition. New York, NY: John Wiley \& Sons, Inc. 
Rackwitz, R., and B. Fiessler. 1976. "Note on Discrete Safety Checking When Using Nonnormal Stochastic Model for Basic Variables." Loads Project Working Session. MIT, Cambridge, MA.

Rackwitz, R., and B. Fiessler. 1978. "Structural Reliability under Combined Random Load Sequences.” In Computer and Structures 9:489-494. Oxford: Pergamon Press.

Reese, L. C., L. A. Cooley, and N. Radhakrishnan. 1984. Laterally Loaded Piles and Computer Program COM624G. Technical Report K-84-2. Vicksburg, MS: Department of the Army, Army Corps of Engineers, Waterways Experiment Station.

Schneider, H. R., and M. A. Schneider. 2013. "Dealing with Uncertainties in EC7 with Emphasis on Determination of Characteristic Soil Properties." Modern Geotechnical Codes of Practice. Edited by P. Arnold, G. A. Fenton, M. A. Hicks, T. Schweckendiek, and B. Simpson. Amsterdam, the Netherlands: IOS Press,

Vanmarcke, E. 1977a. "Probabilistic Modeling of Soil Profiles." ASCE Journal of the Geotechnical Division 103(GT11): 1227-1246.

Vanmarcke, E. 1977b. "Reliability of Earth Slopes." ASCE Journal of the Geotechnical Division 103(GT11): 1247-1265.

Vanmarcke, E. 1980. "Probabilistic Stability Analysis of Earth Slopes.” Engineering Geology 16(Special Issue Mechanics of Landslides and Slope Stability): 29-50.

Vesic, A. S. 1967. "A Study of Bearing Capacity of Deep Foundation.” Project B-189. Atlanta, GA: Georgia Institute of Technology.

Vesic, A. S. 1975. "Bearing Capacity of Shallow Foundations.” Foundation Engineering Handbook. Edited by H. F. Wintercorn and H. Y. Fang. New York: Van Nostrand Reinhold.

Zhang, L., W. H. Tang, and C. W. W. Ng. 2001. "Reliability of Axially Loaded Driven Pile Groups.” Journal of Geotechnical and Geoenvironmental Engineering, ASCE 127(12): 1051-1060. 


\section{Appendix A: Computing Probabilities}

\section{A.1 The Gaussian multivariate spaces}

While design and analysis procedures of structures are well defined in theory, in reality the constituent parameters have many variations, which is the reason for LRFD. Material properties (e.g., compressibility, $x$ position) are typically chosen from a range of values that are pulled from a distribution with certain probabilities. An example distribution may be the discrete histogram idealized in Figure A-1. Each bin in the histogram presents how many times that bin has been selected from a sample data set. Dividing those values by the total number of samples drawn gives a normalized distribution, where the probability of selection of any given bin (PDF) is a fraction of the total set and integrating the area under the PDF gives CDF. For the entire curve, the total area is 1.

Figure A-1. PDF and CDF of histogram data.

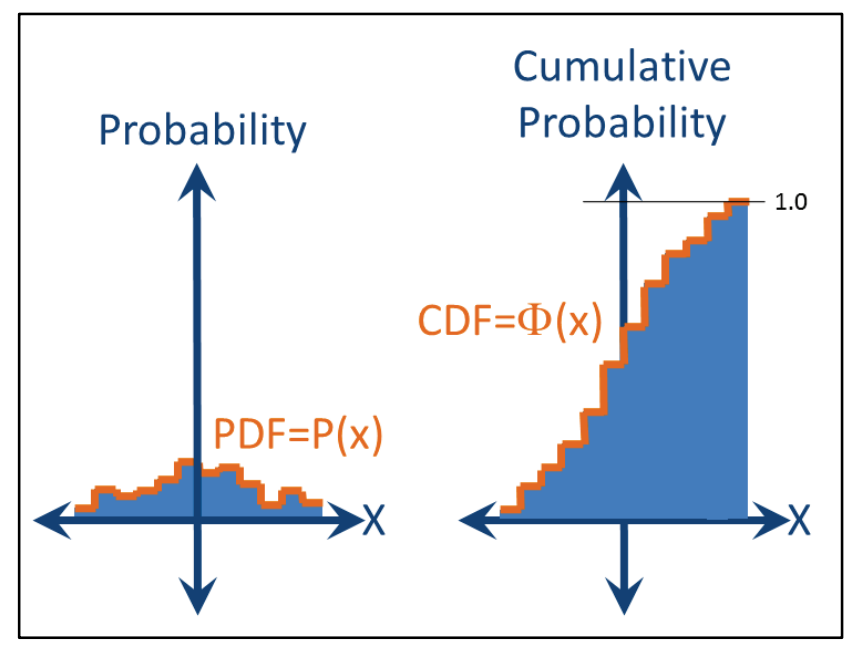

A Gaussian (also referred to as Normal) distribution is given by this equation:

$$
f\left(x \mid \mu, \sigma^{2}\right)=\frac{1}{\sigma \sqrt{2 \pi}} e^{-\frac{(x-\mu)^{2}}{2 \sigma^{2}}}
$$

This function provides numerous advantages when dealing with multiple orthogonal variables in LRFD, so a means to create a Gaussian distribution that approximates other distributions was established by Hasofer and Lind (1974) based on a distribution transform method by Rackwitz and Fiessler $(1976,1978)$. The Hasofer and Lind (1974) procedure does not find the simple mean and standard deviation of another distribution but instead 
maps the CDF of the non-normal distribution to the CDF of a normal distribution to get the best approximate Gaussian distribution with its own mean and standard deviation (Figures A-2 and A-3).

Figure A-2. Histogram data and the Gaussian (normal) distribution.

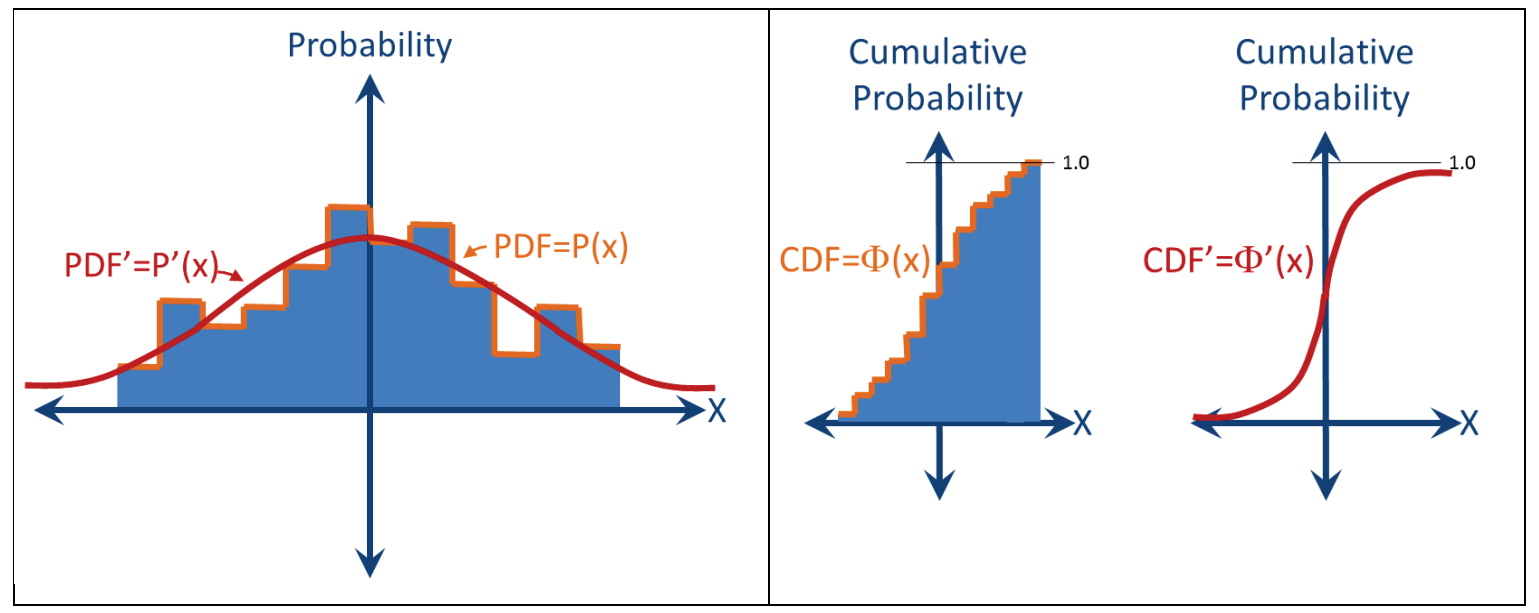

Figure A-3. The $G(X)$ function is used to determine $P U P$.

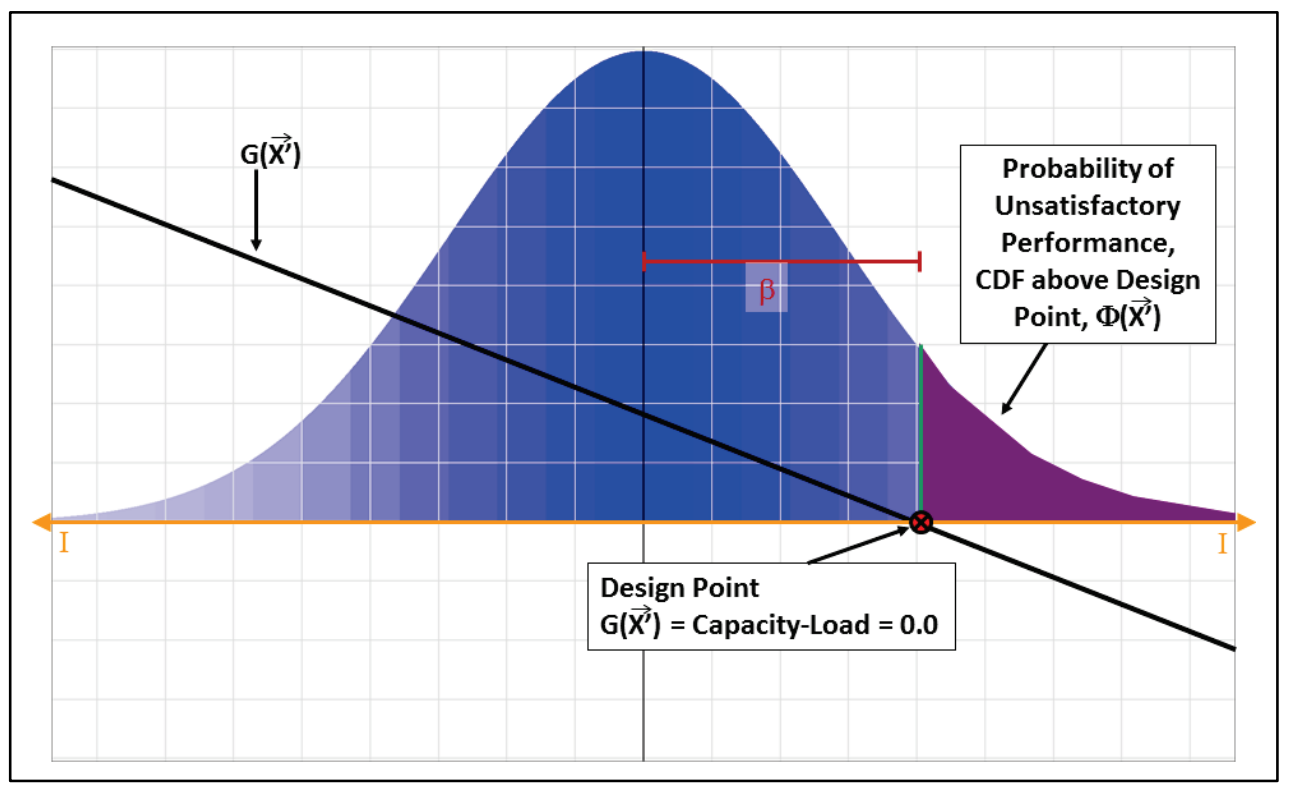

With $N$ uncorrelated and orthogonal variables, an $N$-dimensional multivariate space can be formed from the distributions for those variables. With Gaussian distributions, a transformed space can be created that has a unit distance of $1 \sigma$. The following equation is used to transform the $X$ value of $i^{\text {th }}$ variable of the distribution to the transformed coordinates:

$$
X_{i}^{\prime}=\frac{\left(X_{i}-\mu_{i}\right)}{\sigma_{i}}
$$


and conversely,

$$
X_{i}=X_{i}^{\prime} \sigma_{i}+\mu_{i}
$$

A hyperplane can partition the multivariate Gaussian transformed space into half-spaces. The $N$-space hyperplane has this equation:

$$
0.0=a_{1} * X_{1}+a_{2} * X_{2}+\cdots+a_{N} * X_{N}+c
$$

This hyperplane can also be described by a vector from the origin of the multivariate space to the nearest point on the hyperplane. This vector is perpendicular to the surface of the hyperplane. The vector has length $\beta$ and has unit direction component vector $\alpha$, with $N^{\text {th }}$ axis component of $\alpha_{N}$ (Figure A-4).

Figure A-4. A 2-D example of the PUP region and the hyperplane description using $\alpha$ and $\beta$.

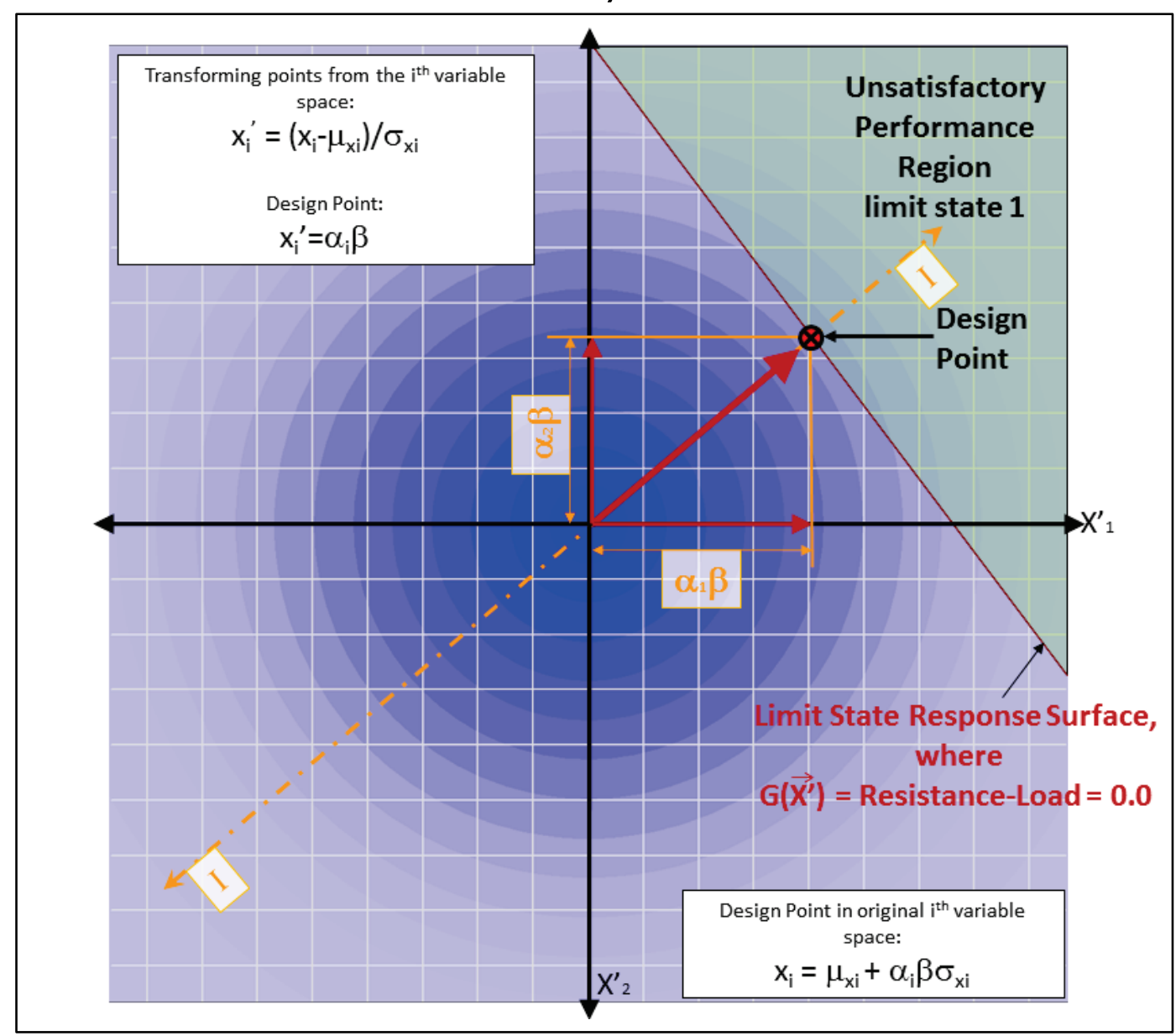


This multivariate, Gaussian transformed space has several properties that make it attractive for determining limit state probabilities, such as is done for reliability analysis and LRFD. The most important property is that, for any hyperplane in the $\mathrm{N}$-dimensional space partitions the space into two half-spaces, the integrated volume of the half-space gives the same result as a the $\operatorname{CDF}(\beta)$ for a normal distribution along the hyperplane's defining vector.

A hyperplane perfectly describes the response surface of a limit state that has linear effects across multiple variable axes. For the purposes of engineering evaluation, it is assumed that each limit state has linear properties and that failure occurs because of non-linear, or plastic, response.

\section{A.2 Limit states and PUP}

For engineering analysis with properties that vary, described by multivariate space, a particular limit state (e.g., foundation sliding) can be described by the multivariate function

$$
g(\boldsymbol{X})=\text { Capacity }- \text { Load }
$$

This function has positive values for satisfactory performance, negative values for unsatisfactory performance, and a value of 0.0 at the response surface, which separates the performance regions (Figure A-5). For probabilities, as discussed above, the response surface is assumed to be hyperplanar. 
Figure A-5. A 2-D example of multiple limit states with closest approach of length $|\beta|$.

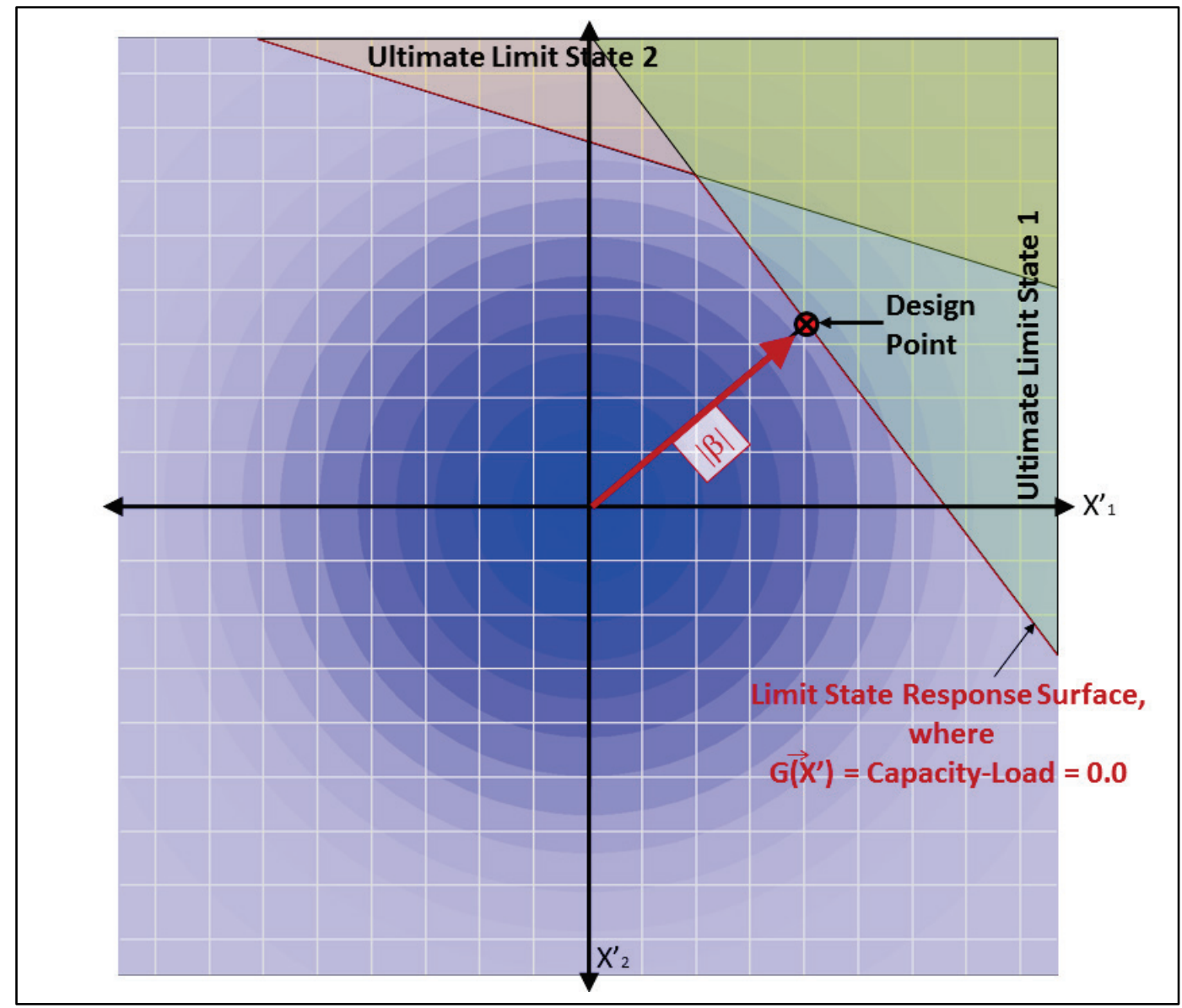


An indicator function can be created with the express purpose of providing a multiplicative identity for $\boldsymbol{X}$ vector values where there is unsatisfactory performance and a value of 0.0 at the response surface of the limit state or less.

$$
i(\boldsymbol{X})=\left\{\begin{array}{l}
0: g(\boldsymbol{X}) \geq 0.0 \\
1: g(\boldsymbol{X})<0.0
\end{array}\right.
$$

The function to calculate PUP for the Gaussian multivariate space $\boldsymbol{X}$ is

$$
p_{U}=\Phi(\boldsymbol{X})=\int_{\mathbb{R}^{N}} i(\boldsymbol{X}) h(\boldsymbol{X}) d \boldsymbol{X}
$$

where $h(\boldsymbol{X})$ is the probability density function for the space and is given by

$$
h(\boldsymbol{X})=\prod_{n=1}^{N} h\left(X_{n}\right)
$$

As noted before, for a single hyperplanar surface in a Gaussian multivariate space, the PUP is the same as the 1-D CDF along the line through the origin that is perpendicular to the plane. For line $l=\alpha \beta$, where $\alpha$ is the directional cosine vector, $p_{U}=\Phi(\beta)$, with $\Phi(\beta)$ being the CDF for the normal Gaussian distribution.

Unfortunately, it is seldom that only one limit state is possible with the engineering design/analysis of structures and especially the USACE hydraulic structures (Section 1.5). In this case, a family of limit states needs to be assessed, with each limit state having a different $g(\boldsymbol{X})$ function. In this case, combining the limit state functions gives a new, nonlinear, overall response surface, described by the boundary of the union of the half-space volumes for each limit state response surface hyperplane. For $L$ limit states,

$$
p_{U}=\bigcup_{n=1}^{L} \Phi_{n}(\boldsymbol{X})
$$

It is important to realize that these half-space sets of data are guaranteed to intersect and overlap if the hyperplane equations are not parallel and may subsume each other if the equations are parallel. This makes it difficult to get an accurate determination of the $P U P$ for multiple limit states. 


\section{A.3 Estimating PUP for multiple limit states}

\section{A.3.1 Bounds Method}

Ang and Tang (1974) suggested an alternative method for estimating the PUP with multiple limit states based on finding the minimum and maximum bounds for the value that the PUP can assume. This estimation is not as accurate as the numerical Euclidean methods but can be found quickly with methods to determine a design point for each limit state, and therefore the equation for the hyperplane and the $\beta$ distance to the hyperplane, resulting in the PUP.

$$
P U P_{L S i}=\Phi\left(\beta_{i}\right)
$$

Each limit state hyperplane divides the Gaussian multivariate space into half-spaces, with one half-space being the satisfactory performance region and the other half-space being the unsatisfactory performance region for that limit state. When there are multiple limit states, these regions in the Gaussian multivariate space overlap and combine. The probability of these combined regions having unsatisfactory performance is the probability of failure given the union of all the regions

$$
P U P \cong p_{u}\left[R_{1} \cup R_{2} \cup \ldots \cup R_{N}\right]
$$

However, as it has been previously described, the union of the regions contains the overlapping volumes of unsatisfactory performance probabilities. This complicates the calculation of the overall PUP.

Because each limit state is described by a hyperplane with shortest distance $\beta$ I from the origin, the lowest bound of the overall PUP can be proven to be the greatest of the individual hyperplane PUPs of the limit states. This is true because, for a set of limit states with parallel hyperplanes, the greatest individual PUP subsumes the volumes of the other individual PUPS.

$$
\max _{i}\left(\Phi\left(\beta_{i}\right)\right) \leq P U P
$$

Parallel hyperplanes can have non-intersecting half-planes of unsatisfactory performance. For two such hyperplanes that do not overlap, the probability of satisfactory performance is the multiplied probabilities of satisfactory performance for each limit state. This implies that an upper 
limit to the PUP can be found by subtracting the combined probability of satisfactory performance for multiple limit states from 1.o.

$$
P U P \leq 1-\prod_{i}\left(1-\Phi\left(\beta_{i}\right)\right)
$$

Equations A.13 and A.14 establish absolute lower and upper bounds for the PUP, but these bounds are for limit states that are not correlated (i.e., have parallel hyperplanes in unimodal sets). In reality, the hyperplanes are seldom parallel and have complex overlapping regions. Therefore, correlation techniques can be used between pairs of hyperplanes (bimodal) to tighten the bounds about the PUP. With more than two hyperplanes (multimodal), the overlapping regions become much more complicated.

According to a decomposition of the union of sets using deMorgan's rule as described in Ang and Tang (1974), the bounding values for PUP using bimodal probabilities is

$$
P U P \geq \max \left[\sum_{i}\left\{P U P_{L S i}-\sum_{j=1}^{i-1} P\left(E_{i} E_{j}\right)\right\} ; 0.0\right]
$$

and

$$
P U P \leq \sum_{i=1}^{N} P U P_{L S i}-\sum_{i=2}^{N} \max _{j<i}\left(P\left(E_{i} E_{j}\right)\right)
$$

Given hyperplane equations for the $\mathrm{i}^{\text {th }}$ and $\mathrm{j}^{\mathrm{th}}$ limit state equations (in 2-D for simplicity):

$$
\begin{aligned}
& g_{i}(\boldsymbol{X})=a_{0}+a_{1} X_{1}+a_{2} X_{2} \\
& g_{j}(\boldsymbol{X})=b_{0}+b_{1} X_{1}+b_{2} X_{2}
\end{aligned}
$$

The limit state response surface is determined by setting these equations to o.o. According to Ang and Tang (1974), in Gaussian multivariate space, the correlation coefficient can be found as the cosine of the dihedral angle $(\theta)$ between these hyperplanes.

$$
\rho_{i j}=\frac{\operatorname{Cov}\left(g_{i}, g_{j}\right)}{\sigma_{g_{i}} \sigma_{g_{j}}}=\cos \theta
$$

The dihedral angle between the planes is equivalent to the angle between the vectors from the origin to the respective design points because each 
hyperplane is perpendicular to its design point vector. The cosine of this angle can be expressed, from the normalized design point vectors $\alpha_{i}$ and $\alpha_{j}$, as

$$
\rho_{i j}=\cos \theta=\boldsymbol{\alpha}_{i} \cdot \boldsymbol{\alpha}_{j}
$$

From Figures A-6 and A-7, observe that $E_{i} E_{j} \supset A$ and $E_{i} E_{j} \supset B$. The probability of $E_{i} E_{j}$ is therefore bounded by

$$
\max [P(A), p(B)] \leq P\left(E_{i} E_{j}\right) \leq P(A)+P(B)
$$

and, by reason of orthogonality,

$$
P(A)=\Phi\left(-\beta_{i}\right) \Phi(-a)=\Phi\left(-\beta_{i}\right) \Phi\left(-\frac{\beta_{j}-\rho \beta_{i}}{\sqrt{1-\rho^{2}}}\right)
$$

and

$$
P(B)=\Phi\left(-\beta_{j}\right) \Phi(-b)=\Phi\left(-\beta_{j}\right) \Phi\left(-\frac{\beta_{i}-\rho \beta_{j}}{\sqrt{1-\rho^{2}}}\right)
$$


Figure A-6. Finding the contribution of area A to overlapping probabilities of $g_{i}(X)$ and $g_{j}(X)$.

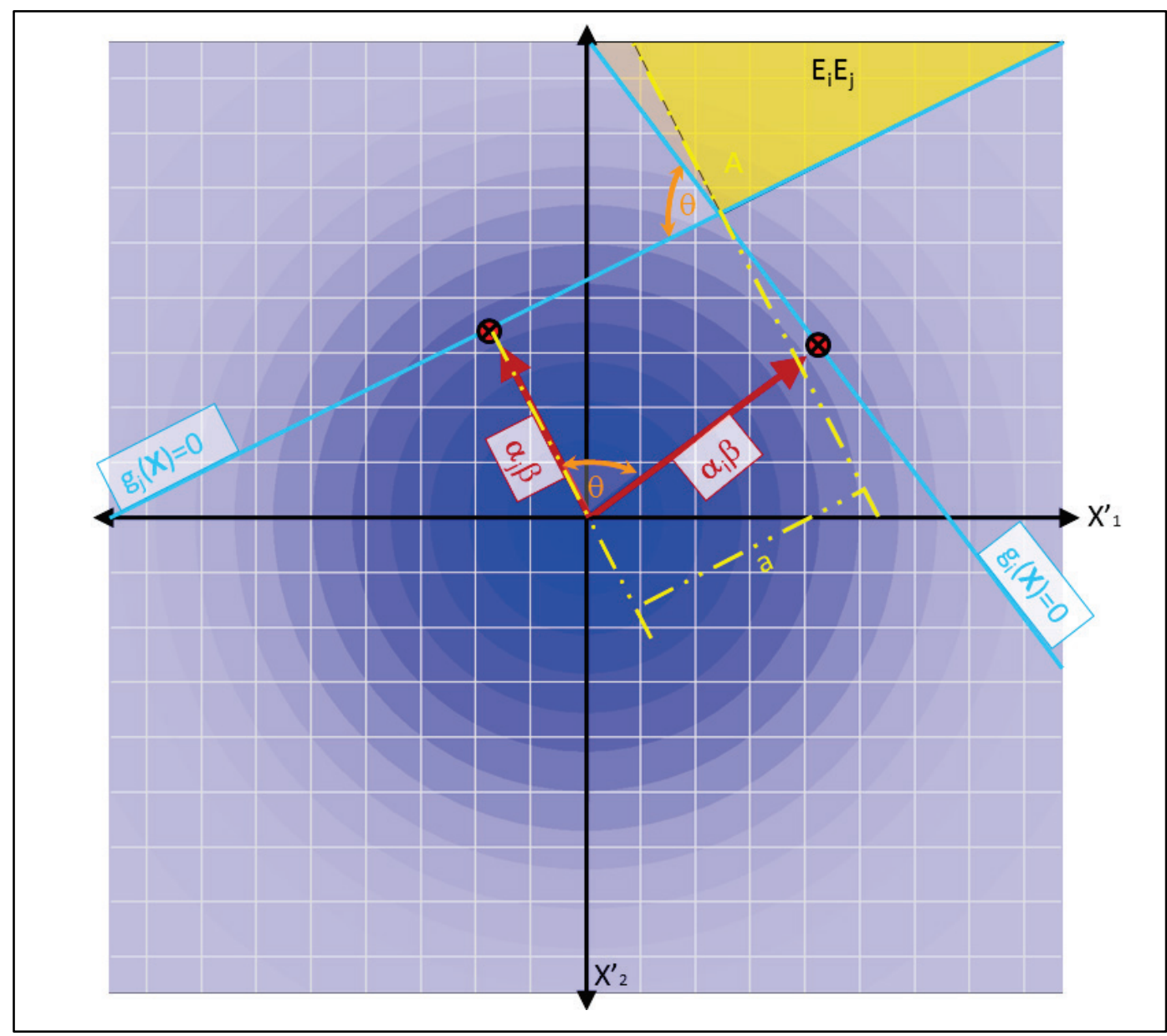


Figure A-7. Finding the contribution of area A to overlapping probabilities of $g_{i}(X)$ and $g_{j}(X)$.

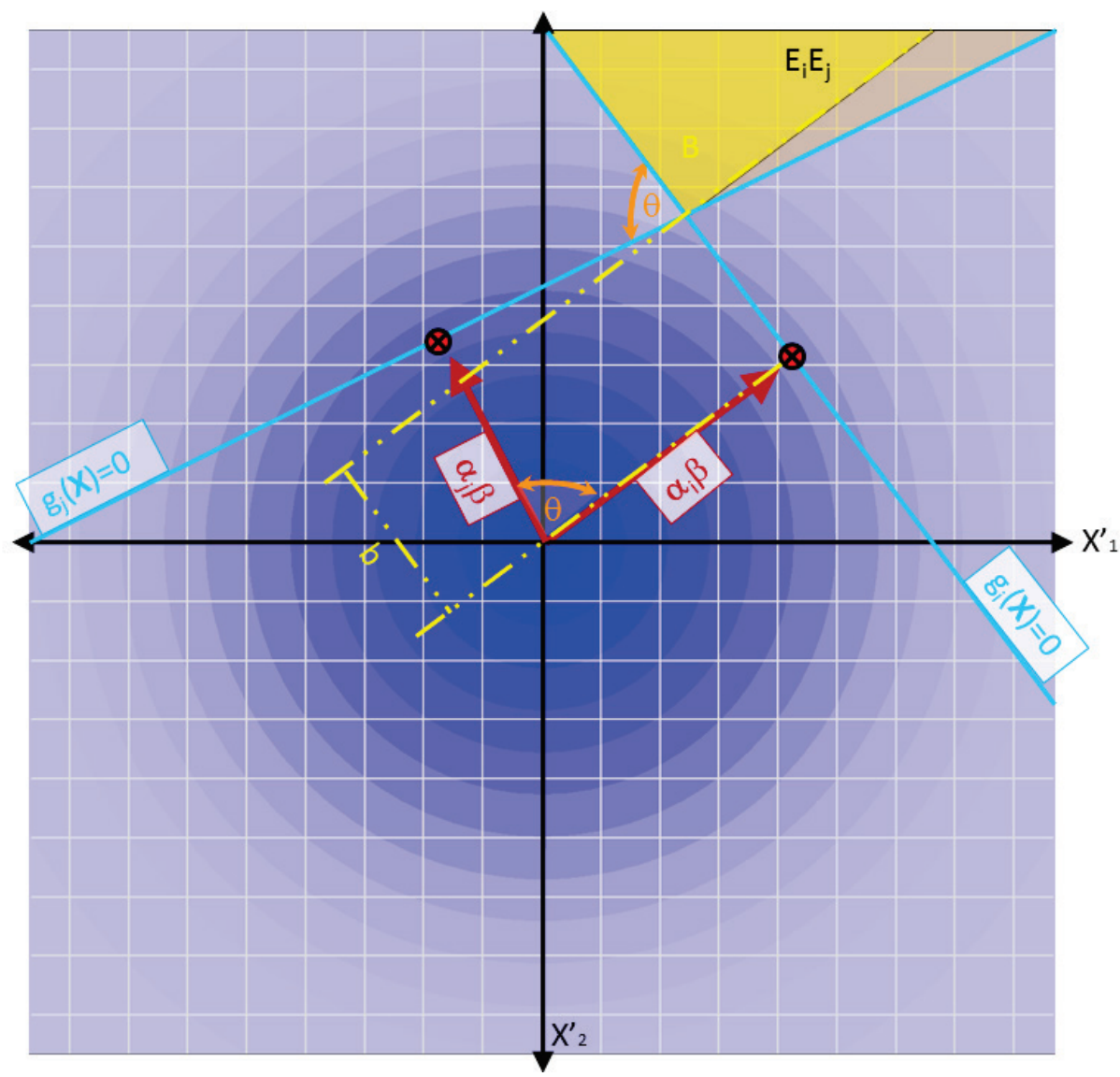

Using the relationships of Equations A.21 and A.22, the lower bound for $P\left(E_{i} E_{j}\right)$, given as

$$
P\left(E_{i} E_{j}\right)=P(A)+P(B)
$$

should be used in Equation A.14 for each pair of limit states, and the upper bound, given as

$$
P\left(E_{i} E_{j}\right)=\max [P(A), P(B)]
$$

should be used in Equation A.15 for each pair of limit states.

The results from the application of these equations is a minimum and maximum range for the overall $P U P$ given multiple limit states using a bimodal analysis, using the bounds of each bimodal probabilities. The PUP 
is guaranteed to be in these ranges, so a reasonable estimate of the actual value of the PUP is the average of the two bounding values, giving a maximum error of half the range.

\section{A.3.2 Euclidean numerical method}

For multiple limit states, it is still possible to integrate the probabilities the spaces using Equation A.7, but there needs to be an adjustment to the indicator function specified in Equation A.6. That indicator function is defined for a single limit state function $g(\boldsymbol{X})$. To work with $Z$ multiple limit states, the overall indicator function must return the multiplicative identity if any of the $Z$ single limit state indicator functions returns with a value of 1 .

$$
\begin{gathered}
i_{Z}(\boldsymbol{X})=1-\prod_{z=1}^{Z}\left(1-i_{z}(\boldsymbol{X})\right) \\
P U P=\Phi(\boldsymbol{X})=\int_{X_{1}} \int_{X_{2}} \ldots \int_{X_{N}}\left\{\begin{array}{c}
i_{Z}\left(X_{1}, X_{2}, \ldots, X_{N}\right) * \\
h\left(X_{1}, X_{2}, \ldots, X_{N}\right) d X_{1} d X_{2} \ldots d X_{N}
\end{array}\right\}
\end{gathered}
$$

It would be possible to approximate the value of the PUP using a Euclidean method. The Midpoint Rule Euclidean method is the one most people remember from early calculus courses, but there are other Euclidean methods (i.e., the Trapezoid Rule and Simpson's Rule) that could give a better estimation and require fewer sub-intervals.

Of course, the Euclidean methods require that the space be divided into a number of segments for each dimension (Figure A-8). Because the Gaussian multivariate space is orthogonal and normalized, the same number of intervals should be applied to each dimension. The greatest rate of change of the function occurs near the origin, so for efficiency, the subdivisions can be placed closer together at the origin than at the extremes. However, since most of the limit states are applied near the tails of the distributions, and therefore at extreme distances $(>3.0 \sigma)$, care should be taken to not spread the subdivisions too far so that accuracy and precision will be maintained. Because the probabilities become extreme at distances of greater than $3 \sigma$, the numerical solution should be satisfactory in the range of $-5 \sigma$ to $5 \sigma$ for each variable. 
Figure A-8. Gridding the Gaussian multivariate space to determine the PUPbased on a single limit state response surface.

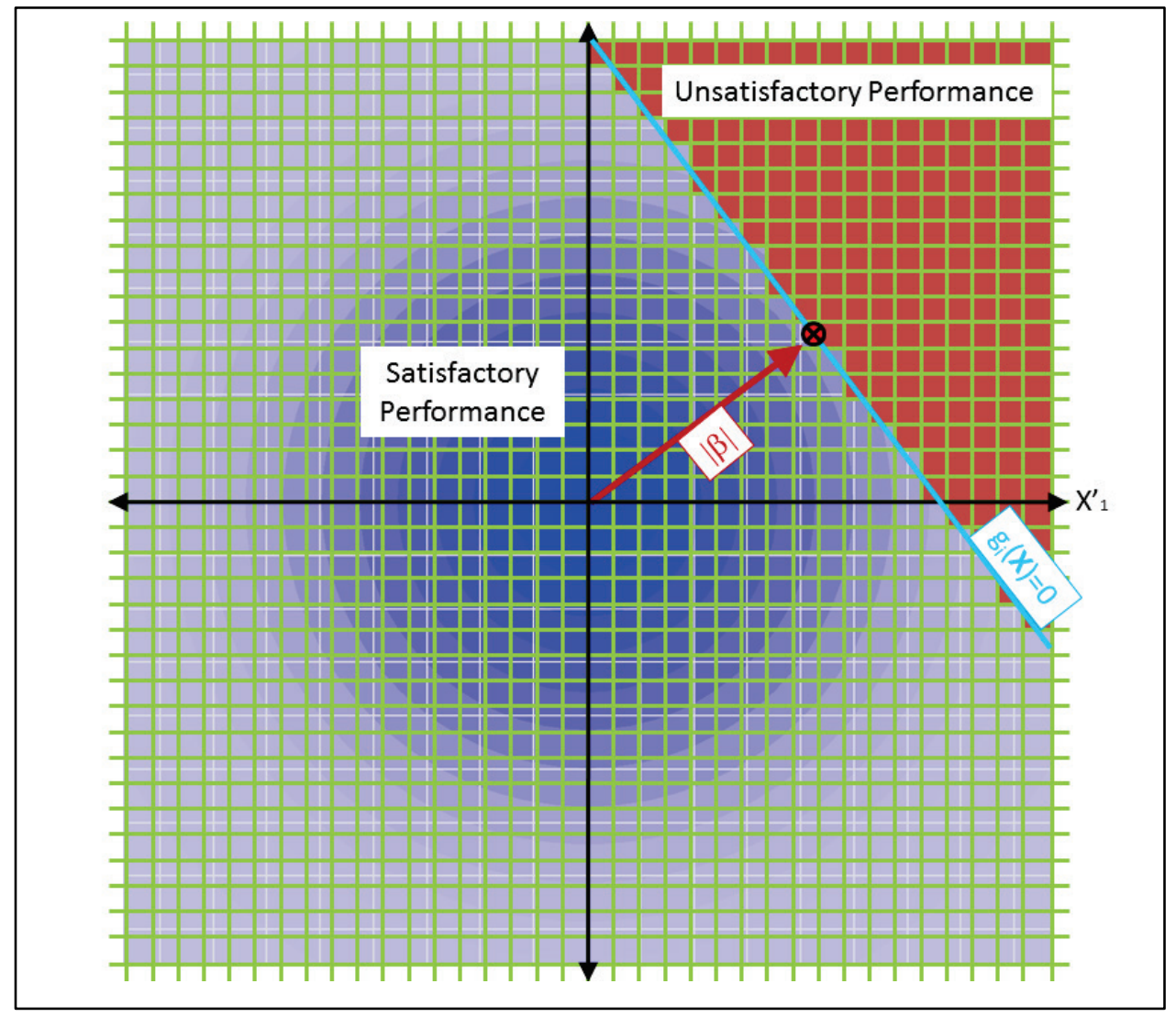

An issue with using the Euclidean approximations is the number of analyses that will need to be run. To evaluate an individual subdivision point, each of the limit state indicator functions will need to be found. If the analysis function is written correctly, these values could be collected on a single analysis execution. However, for a number of sub-divisions $(S)$ and a number of variables $(N)$, the total number of runs that will need to be performed is $N^{S}$. The computational complexity of this method means that it will rarely be tractable for anything less than a high-performance computational platform. Another issue with the numerical integration is that the combined PDF for multiple variables, which are multiplied together, make numbers so small that they outstrip the precision of even double-precision floating point values in programming languages.

\section{A.3.3 Hypersphere numerical method}

Thus far, all of the methods explored have been based on hyperplanes because there is a simple method of determining the CDF of half-spaces divided by the hyperplane in the Gaussian multivariate space. There is 
another property of the Gaussian multivariate space that seems to be overlooked. For any hypersphere (also known as an $n$-sphere) in the space that is centered at the origin and has radius $R$, all of the points on the surface of the hypersphere have the same probability density function value, as shown in Figures A-9 and A-10.

$$
p\left(\boldsymbol{X}_{R}\right)=p\left(R_{X 1}\right) \times p\left(R_{X 2}\right) \times \ldots \times p\left(R_{X N}\right)=p(0)^{N-1} p(R)
$$

Figure A-9 shows that the probability for any thin shell is the same at a set distance from the origin. Figure A-10 shows this thin shell in plan view. The symmetry and the properties of the Gaussian Normal Curve means that the probability at any distance in the multivariate space is the same at all points on the hypersphere surface.

Figure A-9. Symmetric probability for pairs of bins equidistant from the mean, section view.

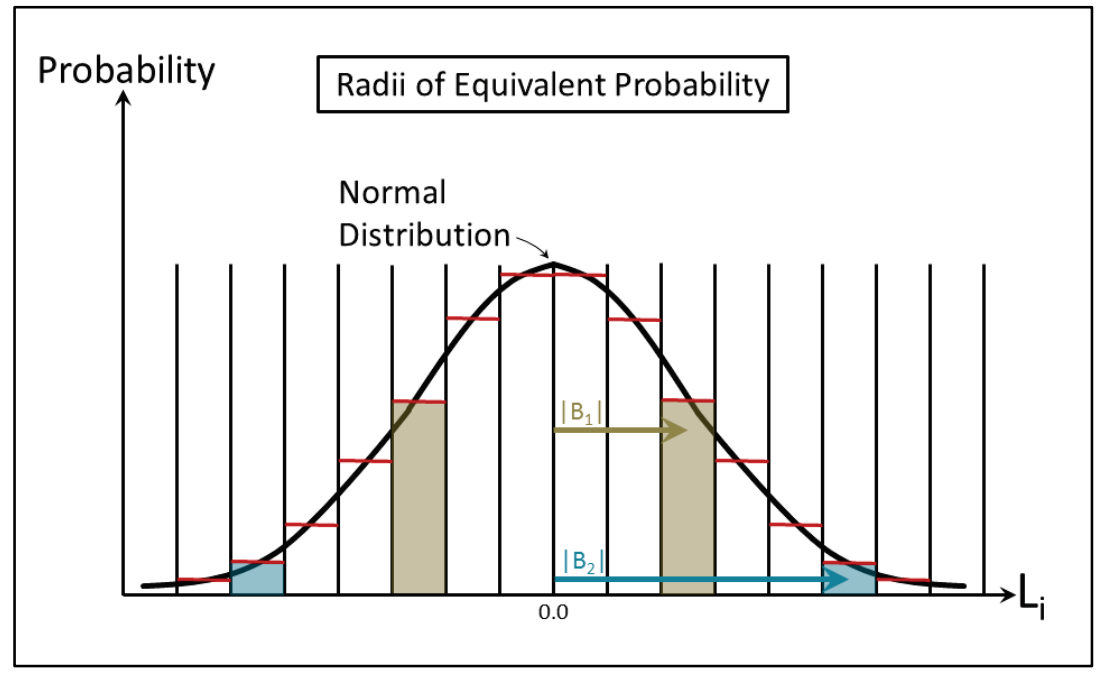


Figure A-10. Symmetric probability of the Gaussian Normal Curve at any distance in the multivariate space at all points on the hypersphere surface, plan view.

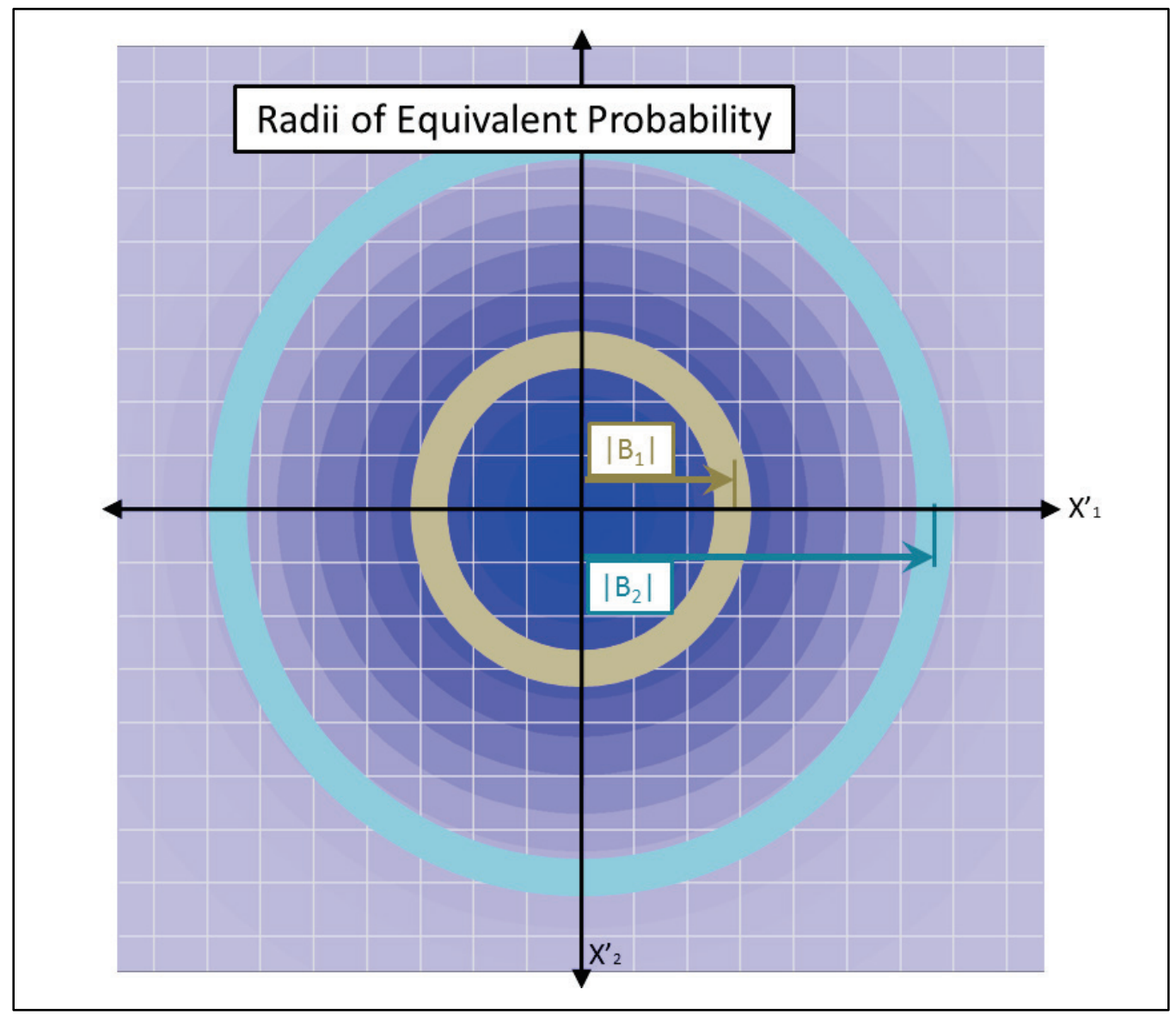

Given that the closed form equation of the surface area for the unit hypersphere with dimension $N$ is

$$
S_{N}=\left[\begin{array}{c}
\frac{2^{\frac{N+11}{2}} \pi^{\frac{N-1}{2}}}{(\mathrm{~N}-2) ! !} \text { for odd } N, \text { and } \\
\frac{2 \pi^{\frac{N}{2}}}{\left(\frac{1}{2} \mathrm{~N}-1\right) !} \text { for even } N
\end{array}\right.
$$

Using concentric shells, the probability of satisfactory performance of the $N$-dimensional hypersphere $(h)$ with radius $R$ in Gaussian multivariate space (Figure A-11) is

$$
P_{h}(R)=\int_{r=0}^{R} S_{N} r^{2} p(0)^{N-1} p(r) d r
$$

which seems much more tractable for a numerical solution, the factorial divisors in Equation A.27 can grow quite large as the number of dimensions 
increases. For a PUP given hyperplanar limit states and $\beta$ radius, it follows that

$$
P U P \leq\left(1-P_{h}(\beta)\right)
$$

Figure A-11. A 2-D demonstration of the hypersphere calculation of PUP.

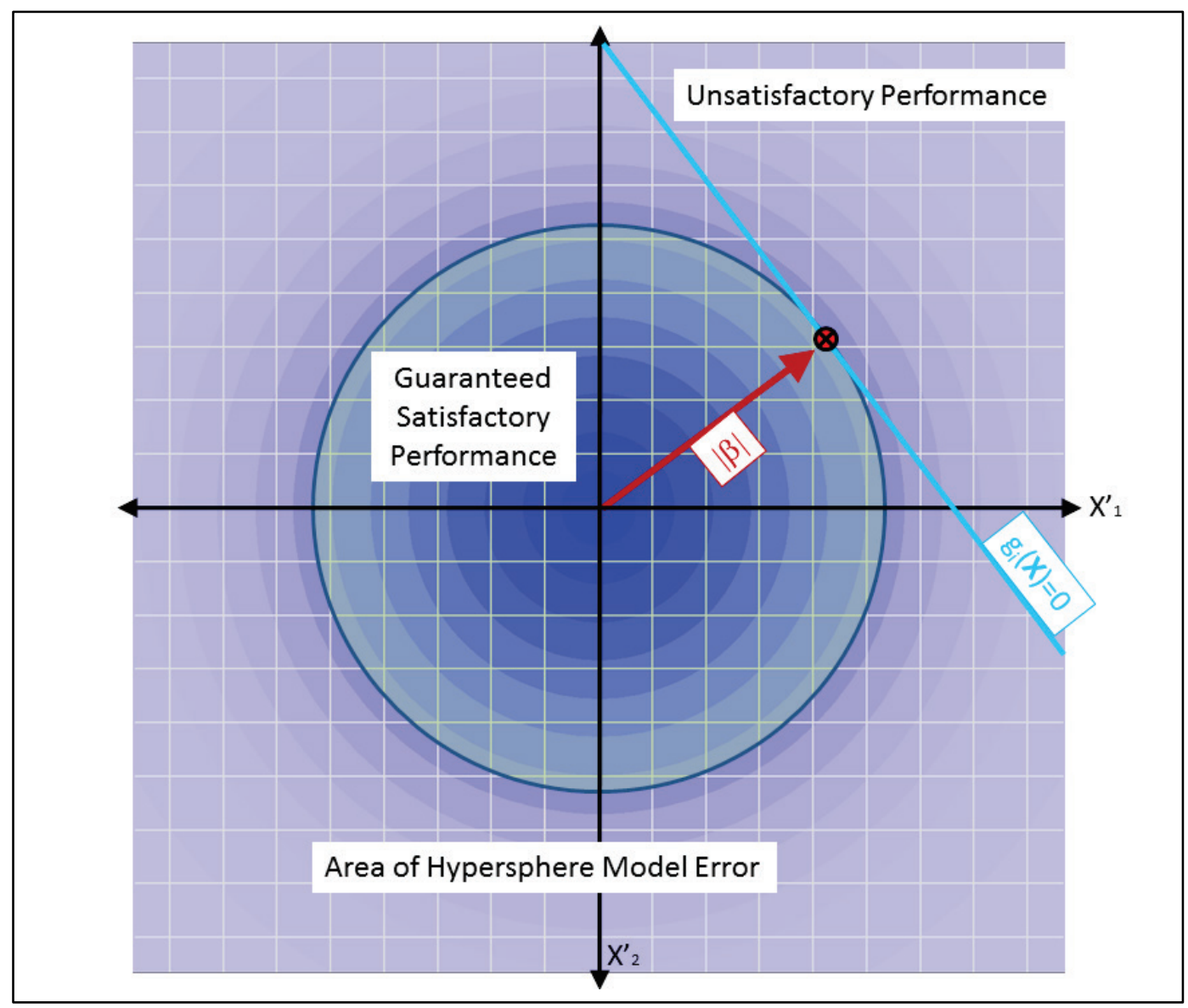

Unfortunately, this approach overestimates the PUP, and the error increases as the radius $\beta$ increases. The error increases because the hyperplane PUP decreases at such a rate that any error is exacerbated at the tails of the distribution. Also, the accuracy of the hypersphere PUP is greatly influenced by the resolution of numerical procedure to determine its values, with greater error occurring at greater $\beta$ values. Of course, higher resolution numerical solutions also become intractable.

Table A-1 shows a quick calculation of error for a two-variable space, with hyperplanar PUP calculations and the numerical approximation of the integral of the hypersphere with different resolution step sizes. Recall that for a two-variable space, the hyperplane is a line and the hypersphere is a 
circle. Table A-2 shows the hypersphere estimation error as a multiple of the actual linear PUP value. Notice that as the hypersphere $\beta$ increases, the error becomes greater. This is because the drop-off of the hyperplanar limit state CDF ( $\Phi(1-\beta))$ drops off more rapidly than the CDF of the area outside the circular area in the opposite half-plane.

Table A-1. PUPvalues for Hyperplanar Limit State and Hypersphere PUPEstimations with increasing accuracy.

\begin{tabular}{|l|l|l|l|l|l|}
\hline$\beta$ & $\begin{array}{l}\text { Hyperplanar } \\
\text { Linear } P U P \\
(I P U P)\end{array}$ & $\begin{array}{l}\text { Hypersphere } \\
\text { PUP(hPUP) }\end{array}$ & $\begin{array}{l}\text { Hypersphere } \\
P U P(h P U P)\end{array}$ & $\begin{array}{l}\text { Hypersphere } \\
P U P(h P U P)\end{array}$ & $\begin{array}{l}\text { Hypersphere } \\
P U P(h P U P)\end{array}$ \\
\hline 0.001 interval) & $(0.0001$ interval) & $\begin{array}{l}(0.00001 \\
\text { interval })\end{array}$ & $\begin{array}{l}(0.000001 \\
\text { interval) }\end{array}$ \\
\hline 1 & 0.15865525 & 0.60604896 & 0.60648246 & 0.60653191 & 0.60653018 \\
\hline 1.5 & 0.0668072 & 0.32446544 & 0.32463372 & 0.32465059 & 0.32465228 \\
\hline 2 & 0.02275013 & 0.1355278 & 0.1353545 & 0.13533991 & 0.13533548 \\
\hline 2.5 & 0.00620967 & 0.04439116 & 0.04398235 & 0.04394257 & 0.04393739 \\
\hline 3 & 0.0013499 & 0.01168391 & 0.01116649 & 0.01111475 & 0.01110957 \\
\hline 3.5 & 0.00023263 & 0.00280223 & 0.00224898 & 0.00219364 & 0.00218811 \\
\hline 4 & 0.00003167 & 0.00095991 & 0.00039792 & 0.00034172 & 0.00033609 \\
\hline 4.5 & 0.0000034 & 0.00066628 & 0.0001027 & 0.00004633 & 0.00004069 \\
\hline 5 & 0.00000029 & 0.00063019 & 0.00006639 & 0.00000999 & 0.00000435 \\
\hline
\end{tabular}

Table A-2. Hypersphere PUPestimate error as a multiple of the actual Hyperplanar PUP.

\begin{tabular}{|l|l|l|l|l|}
\hline $\boldsymbol{\beta}$ & hPUP0.001/IPUP & hPUP0.0001/IPUP & hPUP0.00001/IPUP & hPUP0.000001/IPUP \\
\hline 0.5 & 2.858889618 & 2.860121041 & 2.860258431 & 2.860256421 \\
\hline 1 & 3.81991116 & 3.822643499 & 3.822955181 & 3.822944277 \\
\hline 1.5 & 4.856743585 & 4.859262475 & 4.859514992 & 4.859540289 \\
\hline 2 & 5.957231893 & 5.949614354 & 5.948973039 & 5.948778315 \\
\hline 2.5 & 7.148714827 & 7.082880411 & 7.076474273 & 7.07564009 \\
\hline 3 & 8.655389288 & 8.272086821 & 8.233758056 & 8.229920735 \\
\hline 3.5 & 12.04586683 & 9.667626703 & 9.429738211 & 9.405966556 \\
\hline 4 & 30.30975687 & 12.56457215 & 10.7900221 & 10.61225134 \\
\hline 4.5 & 195.9647059 & 30.20588235 & 13.62647059 & 11.96764706 \\
\hline 5 & 2173.068966 & 228.9310345 & 34.44827586 & 15 \\
\hline
\end{tabular}




\section{A.3.4 Hybrid hyperspherical probabilistic integration method}

The hypersphere numerical method works quickly to determine the probabilities of values occurring within a hypersphere of a defined radius and can be run to generate tables for many different radii hyperspheres for quick lookup. This method could be used to generate a quick estimate of the value to the closest design point for the most likely to occur limit state but will result in a large error region.

The authors of this report have looked at a Euclidean numerical method in Cartesian space for the determination of probabilities with respect to multiple limit states. This concept can be expanded to a polar coordinate space, using

$$
\begin{gathered}
i_{Z}(r, \boldsymbol{\theta})=1-\prod_{z=1}^{Z}\left(1-i_{z}(r, \boldsymbol{\theta})\right) \\
P U P=\Phi(r, \boldsymbol{\theta})=\int_{\boldsymbol{\theta}} \int_{r=0}^{\infty} i_{Z}\left(r, \theta_{1}, \theta_{2}, \ldots, \theta_{N}\right) h\left(r, \theta_{1}, \theta_{2}, \ldots, \theta_{N}\right) d r d \boldsymbol{\theta}(\mathrm{A} .32)
\end{gathered}
$$

A numerical solution could transform the polar coordinates to Cartesian coordinates for simplification of probability calculation. This means that the $i_{Z}()$ function can be determined from the Cartesian equation given in Equation A.25. The probability function $h()$ is the same as Equation A.8. The rest of the equation deals with the approximation of volume occupied by a polar coordinate described shell element of $d r$ thickness and all of its sweep angles. Because dimensionless angles are used for these sweep angles in multiple dimensions and the angles interact within polar coordinates, the definition of $d \boldsymbol{\theta}$ needs to be rederived for each number of dimensions. The following two equations show the polar integration equations for two and three dimensions, respectively:

$$
\begin{array}{r}
P U P=\Phi(r, \theta)=\int_{\boldsymbol{\theta}=\mathbf{0}}^{2 \pi} \int_{r=0}^{\infty} i_{Z}(r, \theta) h(r, \theta) d r(r d \theta) \\
P U P=\Phi\left(r, \theta_{1}, \theta_{2}\right)= \\
\int_{\theta_{2}=0}^{2 \pi} \int_{\theta_{1}=0}^{\pi} \int_{r=0}^{\infty}\left\{\begin{array}{c}
i_{Z}\left(r, \theta_{1}, \theta_{2}\right) h\left(r, \theta_{1}, \theta_{2}\right) * \\
d r\left(r d \theta_{1}\right)\left(r \sin \left(d \theta_{1}\right) d \theta_{2}\right)
\end{array}\right\}
\end{array}
$$

The length term $r$ needs to be multiplied by each angular delta to express the shell element arc-distance for that angular sweep. For the 3-D Equation A.32b, the total sweep of angle $\theta_{1}$ is reduced to make the functions one-to-one, much like latitude and longitude are described on a 
sphere. Figure A-12(a,b) show why the reduction of distance for the $d \theta_{2}$ term is required for polar coordinates. These figures use more conventional notation for 3 -D axes and angles with $X, Y$, and $Z$ axes representing $X_{1}, X_{2}$, and $X_{3}$ variable space axes, respectively, and $\phi$ and $\theta$ representing the $\theta_{1}$ and $\theta_{2}$ variable space angles, respectively. The equation derivations for Equation A.32 become more involved as more variable dimensions are included.

Figure A-12. Calculating dimensions for integrating spherical coordinate 3-D space.

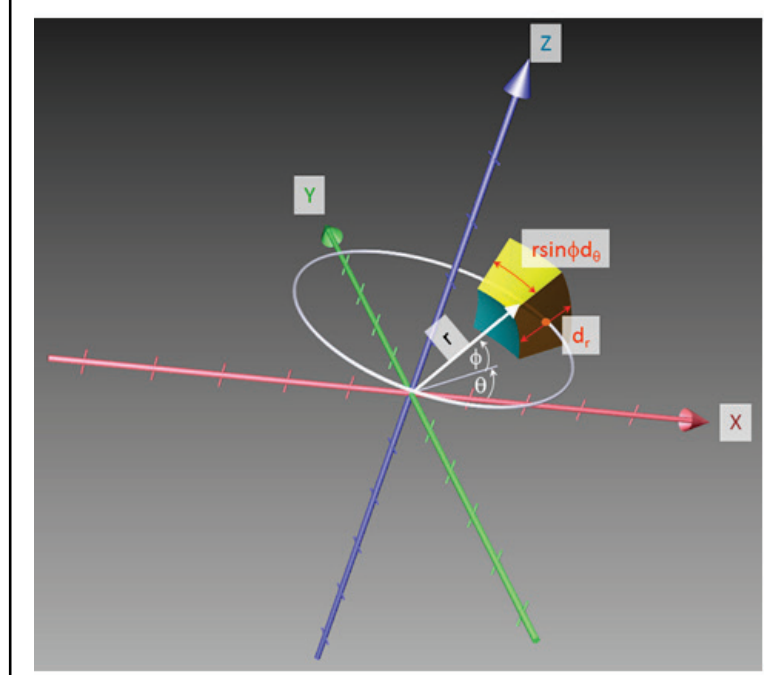

(a)

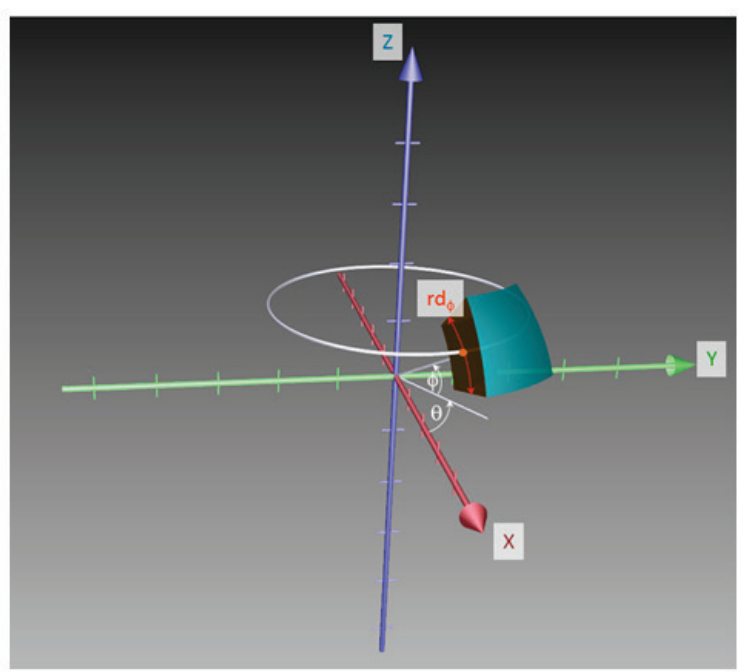

(b)

A full numerical solution along these lines would have the same issues of exponential time growth as the Euclidean method. Recall that the numerical solution could be considered complete when $r$ equals $5 \sigma$ because the probabilities are extremely small past that limit.

It is certainly possible to minimize calculation time by marrying the hypersphere calculation method with the polar coordinate method for calculating the probabilities of multiple limit states in the Gaussian multivariate space. Given the design point for the highest probability limit state defined by $\beta \alpha$, the probability of satisfactory performance would be the hypersphere probability of radius $\beta$ combined with 1 minus the polar $P U P$ with $r$ ranging from $\beta$ to 5 , thus limiting the polar search to a somewhat manageable area. Of course, the PUP is 1 minus the probability of satisfactory performance.

Another performance increase can be implemented if the limit states are guaranteed to be hyperplanar. For the hyperplanar limit states, the ASM 
method calculates the distance from the origin $\beta$ and the unit directional cosine vector $\alpha$ from the origin to the hyperplane which is normal to the surface of the hyperplane. For multiple limit states, the limit state with the minimum $\beta$ value has the highest PUP. The $i_{Z}($ ) function returns a value of o for every point within that minimum $\beta$ value radius hypersphere. Therefore, it is only necessary to integrate from this minimum $\beta$ value to 5 to get the PUP.

If a level of precision is specified by establishing the accuracy to a specific decimal place, another performance increase can be made by using the fact that the volume of hyperspherical shells increases with constant radial increments raised to the power of the number of dimensions $n$ of the hyperspace while the normal distribution values get smaller by a scale factor times $\mathrm{e}^{1 / n}$. The normal distribution values at the tails of the distribution decrease faster than the volume of the hyperspherical shell grows.

Figure A-13a shows the exponential growth of hyperspherical shell volumes as the radius increases, with radial increment 0.001. Figure A-13b shows the normal distribution PDF value assigned for each of these hyperspherical shell radii. Figure A-13c shows the cumulative probability for the volume of the hyperspherical shell elements, computed by multiplying the shell volume by its center radius PDF value. The resulting curve shows that at the beginning, the increase of hyperspherical shell volume from volume o drives the shape of the curve until the PDF distribution drop off exceeds the rate of growth. At that point, the curve begins to decrease with the tails of the distribution. 
Figure A-13. Distribution plots with respect to radius for 3-D sphere: (a) sphere shell volume, (b) point distribution probability, (c) probability density for the sphere shell, and (d) number of divisions of the sphere density where each shell division would have $1.0 \mathrm{e}-10$ probability.

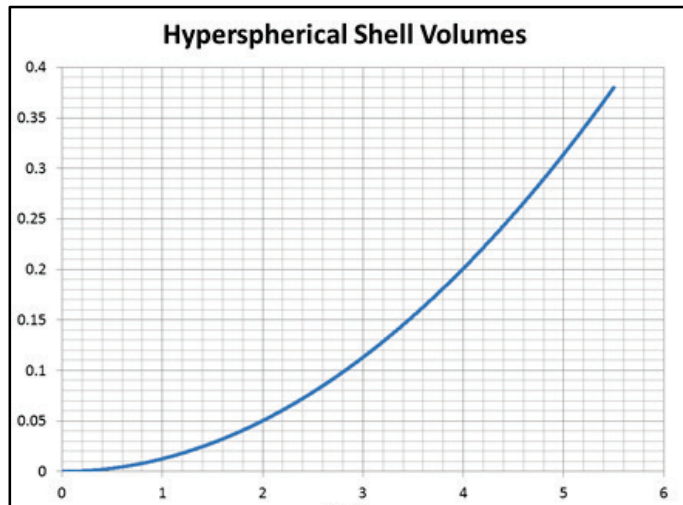

(a)

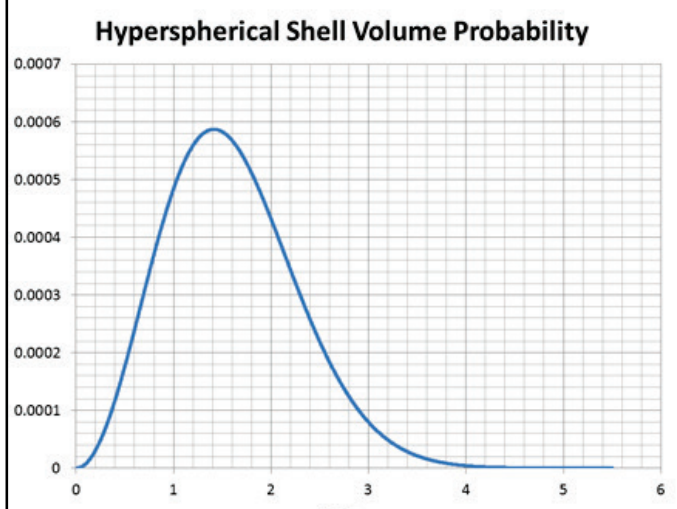

(c)

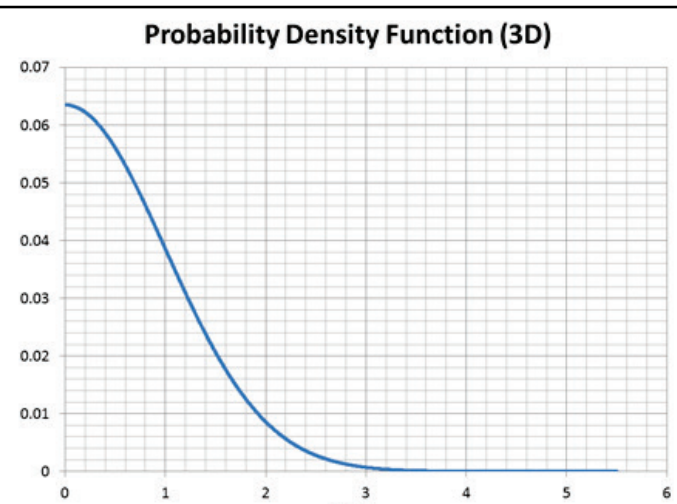

(b)

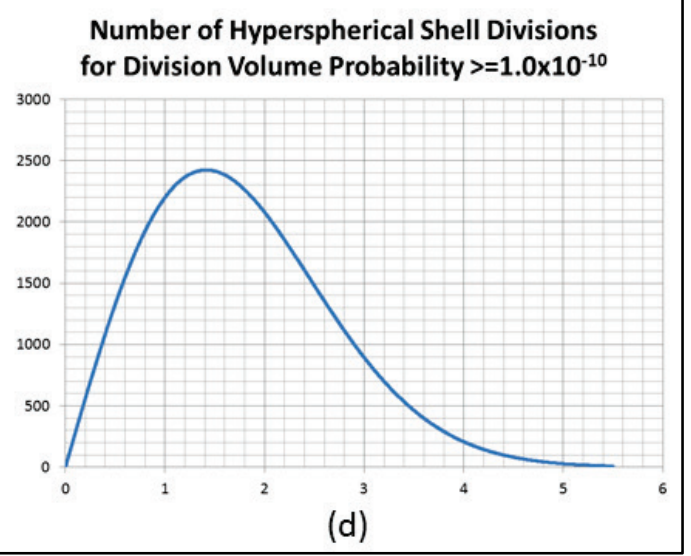

From these curves (Figure A-13 a through c), it can be inferred that, if equal divisions were made of each hyperspherical shell, then each division's volume probability would be equivalent. Further, these divisions (based on angular intervals) can be chosen to be equivalent or slightly less than a specified volume probability based on the precision of solution desired, simply by setting the number of divisions of the hyperspherical shell to the mathematical ceiling of the volume probability of the entire hyperspherical shell by the specified volume probability desired. For the beginning of the curve and the ends of the curve where the volume of the hyperspherical shell is extremely small and the tails of the distribution give extremely low probabilities, respectively, a minimum value for the number of divisions can be set to ensure that limit state values are accurately found. The number of divisions, $n_{\text {angle, }}$, should not drop below a threshold based on the number of variable dimensions (NDim). Samples must be taken at least in every quadrant of the space and each variable dimension doubles the number of quadrants. Therefore, the minimal number of samples should be $2^{\text {NDim }}$ and 
$n_{\text {angle }}$ should not be allowed to be less than $2^{\text {NDim }}$. For an example problem with five dimensions, the minimal value for nangle will be 32. Figure A-13d shows the number of shell divisions so that each division has a volume probability of $1.0 \times 10^{-10}$. Summing these divisions should give the entire ring probability to at least the tenth digit of precision. In truth, there are small errors introduced for three of more dimensions because of polar coordinate integration, so higher precision divisions will be needed to ensure the numerical precision. From this curve, the level of integration is varied as the radius increases.

\section{A.4 Using an estimation of PUP to establish LRFD factors}

Because the structural probability of usability is based on the lifetime of the structure, the PUP can be used to establish the design limits for these structures. Under applied load cases, the design points for several limit states can be determined as a distance (in standard deviations) and direction from the mean characteristics of the structure, $\beta \alpha$. An example of this is shown in Figure A-14. The expected overall PUP value can be set to a limit [e.g., $\Phi(3.5)$ with $\beta=3.5$ ], which guarantees the structure's lifetime integrity probability. 
Figure A-14. Example limit states and $\beta$ s for unfactored loads and resistances.

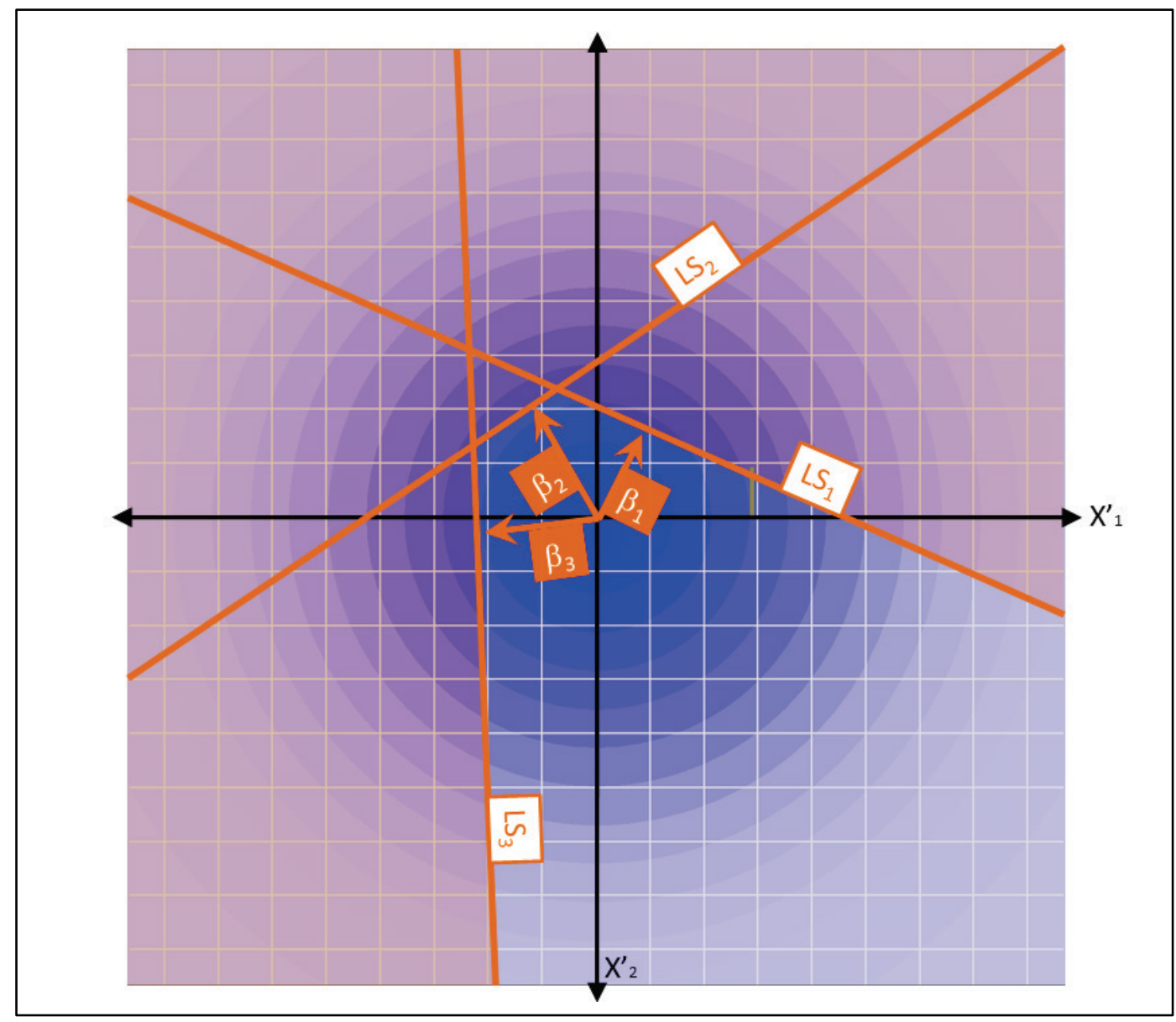

There are two ways to envision that a scale factor can be applied to the load(s) applied to a structure during its structural design: The first method assumes that the load and response directions do not change. In this case, each variable axis has the same universal scale applied (Figure A-15). Because the factor is applied uniformly, the limit states maintain their relative positions and angles to each other in the Gaussian multivariate space. Once the limit states have been determined for the unscaled design load, the system PUP can be considered to be a function of the single applied scale factor $(S)$ to the system.

$$
P U P=f(S)
$$


Figure A-15. A uniform scale factor $(S)$ is applied to the $\beta$ s for the limit state design points.

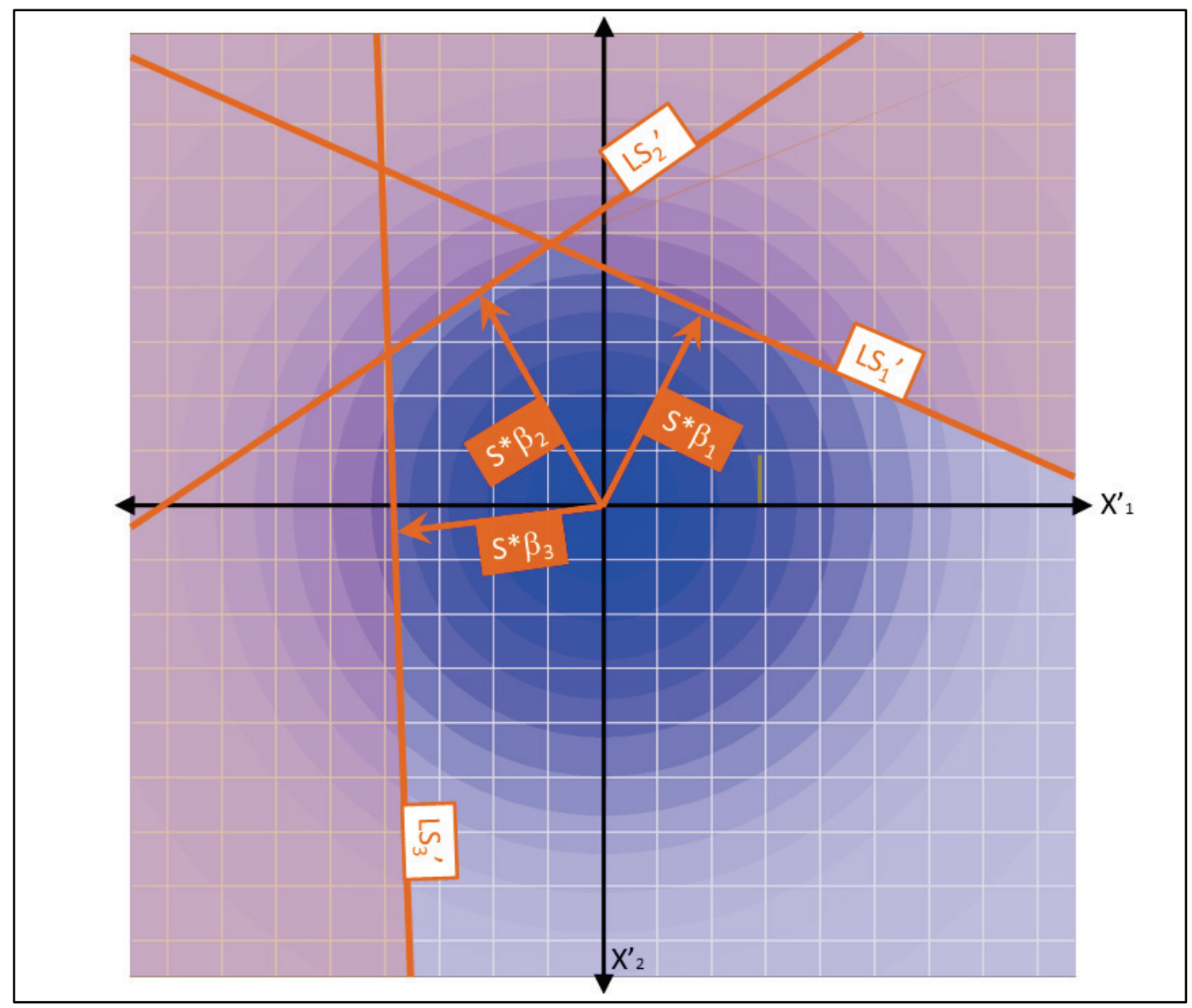

For this equation, $f(S)$ is a result of one of the multivariate, multi-limit state estimation techniques discussed in the previous section. The purpose is to make the calculated PUP approximately equivalent to the established limit [e.g., $\Phi(3.5)$ with $\beta=3.5$ ]. Each limit state response surface is described by

$$
\beta_{i} \boldsymbol{\alpha}_{\text {-original }}=\left(\beta_{\text {i-original }} \times S\right) \boldsymbol{\alpha}_{i-\text { original }}
$$

Since the PUP can described by a function of a single variable $(S)$, a Newtonian or secant-based numerical method can be used to adjust the design points (by changing the scale) until the solution approaches the limit set for the overall system PUP, in an efficient way.

The second method to apply scale factors is more complicated but can give a better overall design because all of the variables are considered independently. To balance the design to the load and intended probability 
of failure, the initial attempt at the design must find the hyperplanar definition vectors for all of the limit states, $\beta$ i-originaldi-original (Figure A-14). When these hyperplanar descriptions are discovered, factors can be applied to the minimum $\beta$ value limit state that extends the distance from the origin in the Gaussian multivariate space to the limit state hyperplane, thus lowering the PUP for that limit state. However, this factor affects all of the limit states to varying degrees as each variable axis is extended to varying degrees by the minimum limit state's directional cosines (e.g., $\alpha_{i \text {-original_XX) }}$. This is shown in Figure A-16. In this case, Equation A.34 is interpreted in a different way:

$$
\beta_{i-\text { original }} \boldsymbol{\alpha}_{i}=\beta_{\text {i-original }}\left(\boldsymbol{\alpha}_{i-\text { original }} \times S\right)
$$

Given this, each variable axis has its own scale factor

$$
S_{X i}=\alpha_{\text {i-original_Xi }} \times S
$$

For this situation, the PUP is a function of the scale factors for each of the variables.

$$
P U P=f\left(S_{X 1}, S_{X 2}, \ldots, S_{X N}\right)=f(\boldsymbol{S})
$$

Because of correlation between the limit state response surfaces, the concern would be that these functions can lead to local minima and maxima in a root finding because of the new slope calculations. Testing will need to be performed to find if these issues confound root-finding numerical algorithms. 
Figure A-16. Applying variable axis scale factors to adjust probabilities. A scale factor applied to Limit State 1 causes slope changes in the response surfaces for Limit State 2 and 3.

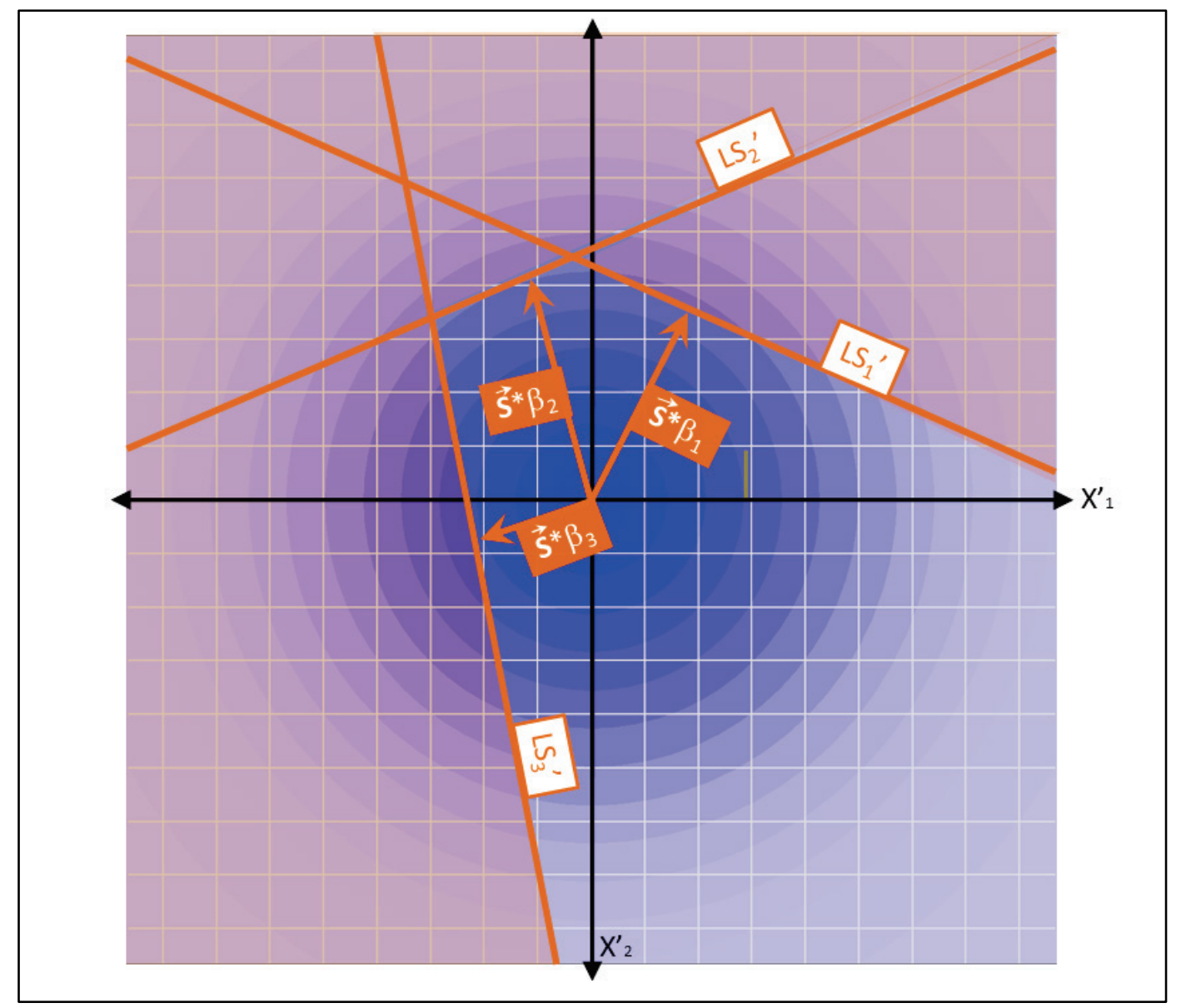

\section{A.5 Validating PUP numerical estimation methods}

To validate the models for PUP estimation, evaluate the inputs that will lead to an increase in accuracy, and determine the resources (namely, time) that will be needed to do these estimations, simple test cases were created. These test cases were performed with two variables and had three overlapping limit states. These tests were all performed on a Dell Precision T7610 computer using Python 2.6 language for purposes of timing.

The first test case had two limit states that were perpendicular to the $X_{1}$ variable axis at distance 1.0 and -1.2 along the axis. The third limit state was perpendicular to the $X_{2}$ variable axis at distance 1.3 along the axis. For all three limit states, the unsatisfactory performance was in the half-space away from the origin of the Gaussian multivariate space. This can be seen in Figure A-17. 
Figure A-17. The first test case for PUPestimation.

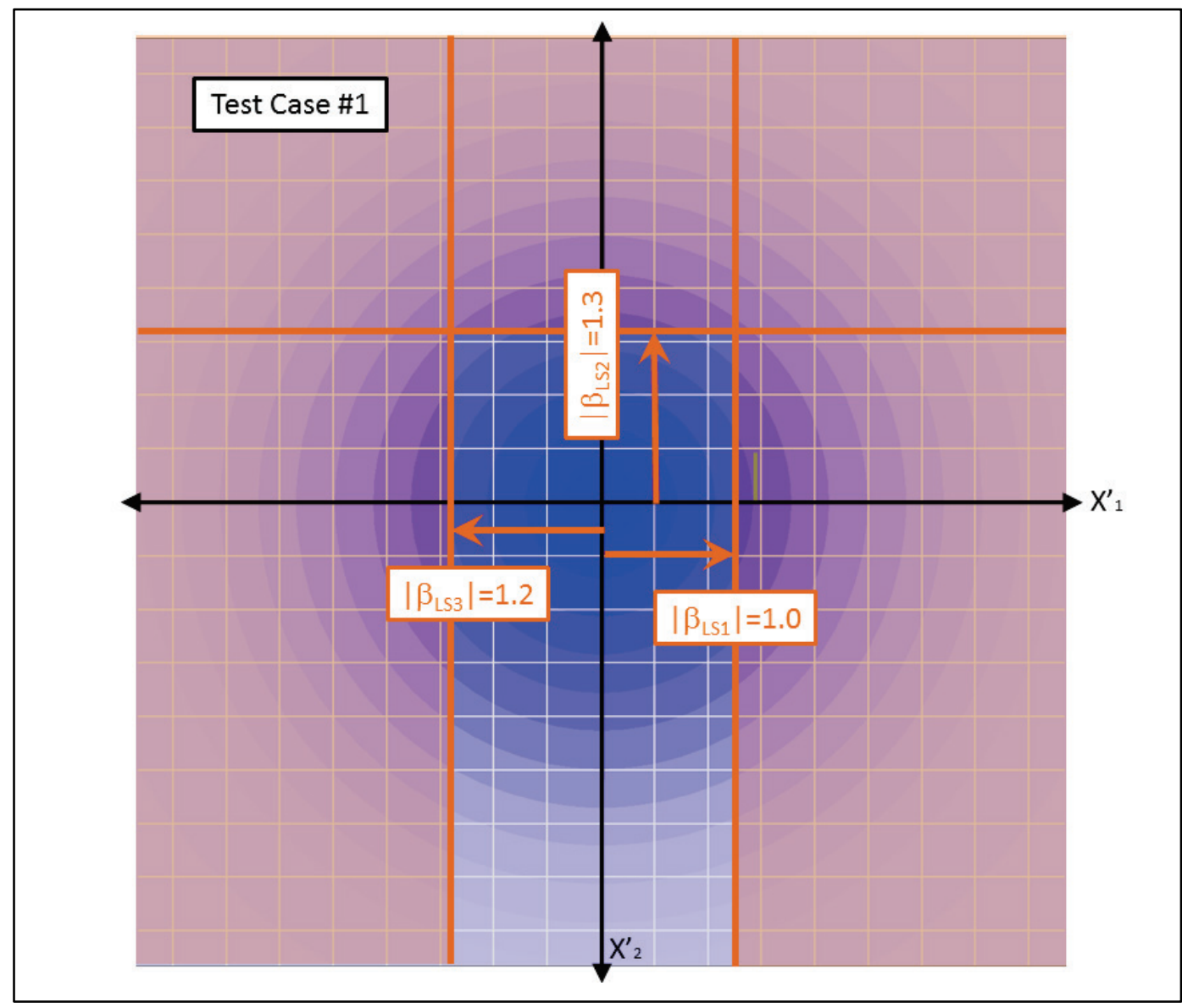

Because the limit states are perpendicular or parallel, the limit states are completely uncorrelated. This simplifies the calculation of the actual PUP. The probability of the overlapping area of two limit state half-spaces is the multiplication of each limit states' PUP. This result is shown in Equations A.21 and A.22, when $\rho=0.0$. This means that

$$
P\left(E_{i} E_{j}\right)=\Phi\left(-\beta_{i}\right) \Phi\left(-\beta_{j}\right)
$$

Therefore, for the first simple test case, the PUP is given by

$$
P U P=\left\{\begin{array}{c}
\Phi\left(-\beta_{L S 1}\right)+\Phi\left(-\beta_{L S 2}\right)+\Phi\left(-\beta_{L S 3}\right)- \\
\Phi\left(-\beta_{L S 1}\right) \Phi\left(-\beta_{L S 3}\right)-\Phi\left(-\beta_{L S 2}\right) \Phi\left(-\beta_{L S 3}\right)
\end{array}\right\}=0.34402870
$$

Listings A.1 and A.2 show the result of performing a simple numeric integration across the test case from -5 to 5 in each dimension of the Gaussian multivariate space with step resolutions of 0.01 and 0.001 , respectively. The first few lines show the calculation of the PUP as 
discussed previously. The estimated value of PUP with resolution 0.01 from the integration is 0.3414388654 and took $154 \mathrm{sec}$ to integrate. This value is within $0.2 \%$ of the actual calculated PUP value. The estimated value of PUP with resolution 0.001 from the integration is 0.3437685994 and took $16,127 \mathrm{sec}$ to integrate. This value is with $0.05 \%$ of the actual calculated $P U P$ value. While the additional accuracy can make slight changes at the tails of the distribution, the solution time means that the 0.01 resolution numerical integration is preferred. 
Listing A.1. Output from Euclidean numerical integration with step size 0.01 .

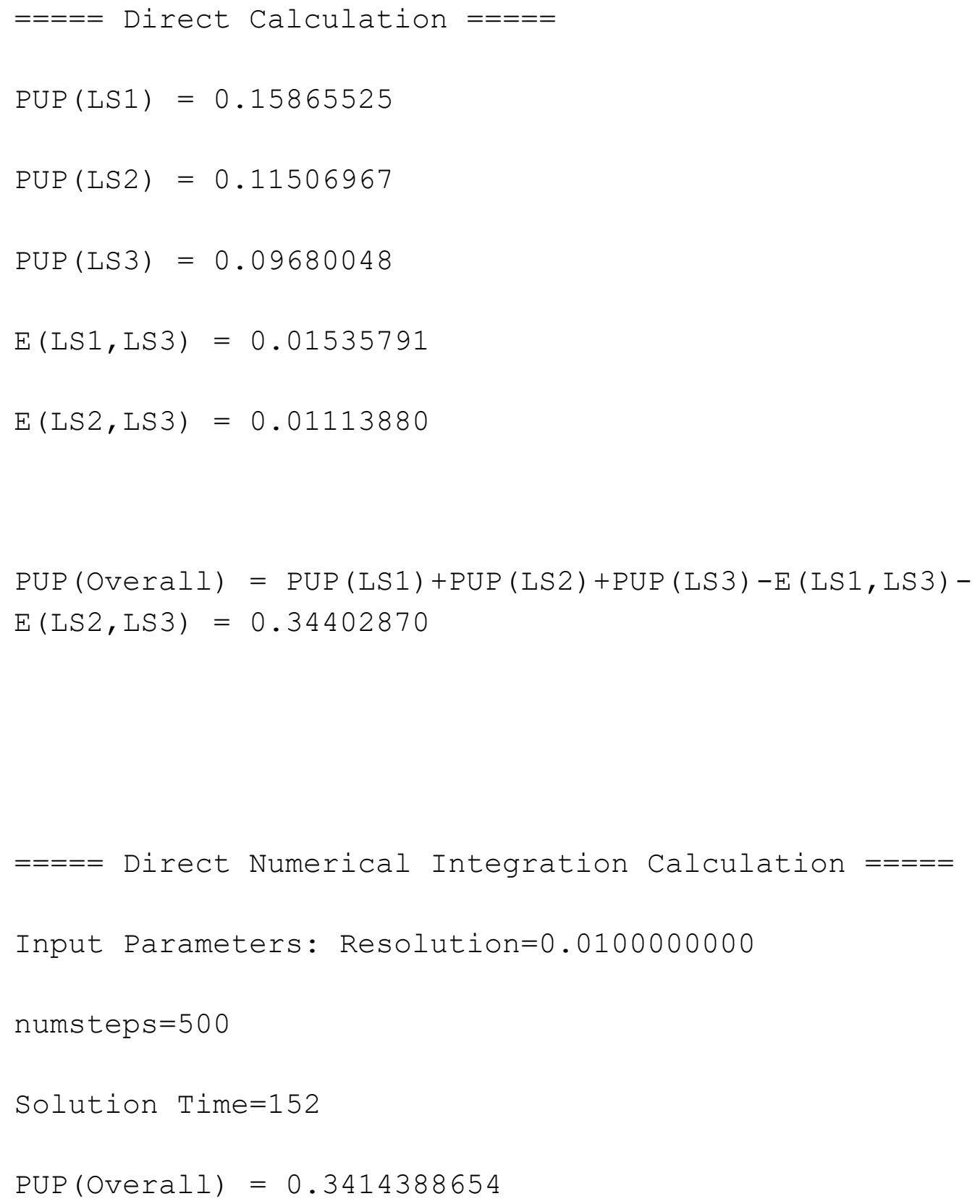


Listing A.2. Output from Euclidean numerical integration with step size 0.001.

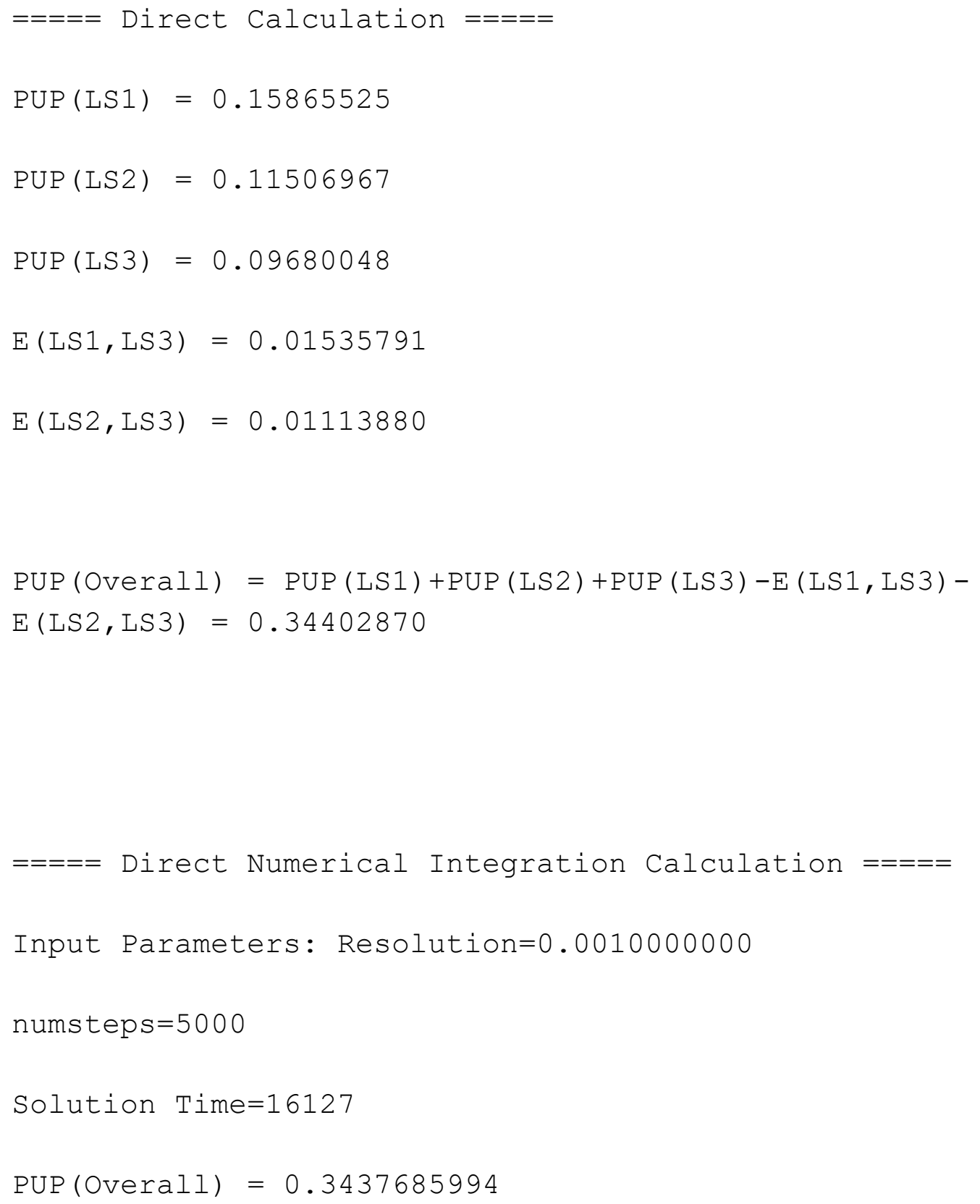


Listing A.3 and A.4 show the results of pure polar integration for the same test case. Recall that polar integration can take advantage of the fact that any point on the hypersphere has the same probability in the Gaussian multivariate space for a significant speed-up. The integration is performed from the center to a hypersphere of radius 5 . The angle step is 0.01 for both runs and the radius changes by steps of 0.01 and 0.001 , respectively. The first few lines show the calculation of the PUP as discussed previously. The estimated value of $P U P$ with radius resolution 0.01 from the integration is 0.3438739669 and took $217 \mathrm{sec}$ to integrate. This value is within $0.05 \%$ of the actual calculated PUP value and has better accuracy than the Euclidean numerical integration does with 0.001 step resolution. The estimated value of PUP with resolution 0.001 from the integration is 0.3440204733 and took $2,036 \mathrm{sec}$ to integrate. This value is with $0.003 \%$ of the actual calculated PUP value. While the additional accuracy can make slight changes at the tails of the distribution, the solution time means that the 0.01 resolution adaptive hyperspherical Gaussian probability numerical integration is preferred and is better than the Euclidean numerical integration. 
Listing A.3. Output from adaptive hyperspherical Gaussian probability numerical integration with radius step size 0.01 and angle step size 0.001 .

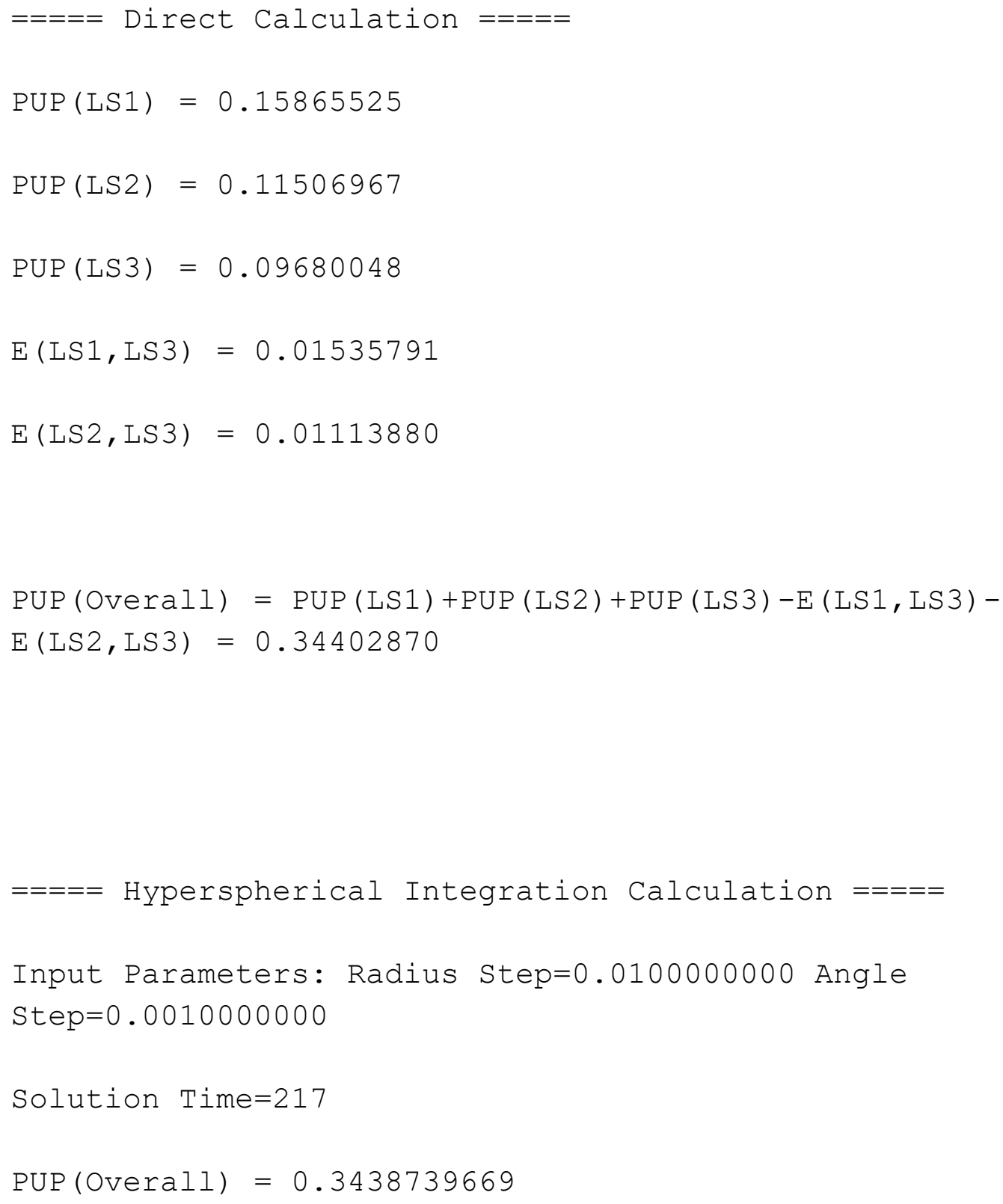


Listing A.4. Output from adaptive hyperspherical Gaussian probability numerical integration with radius step size 0.001 and angle step size 0.001 .

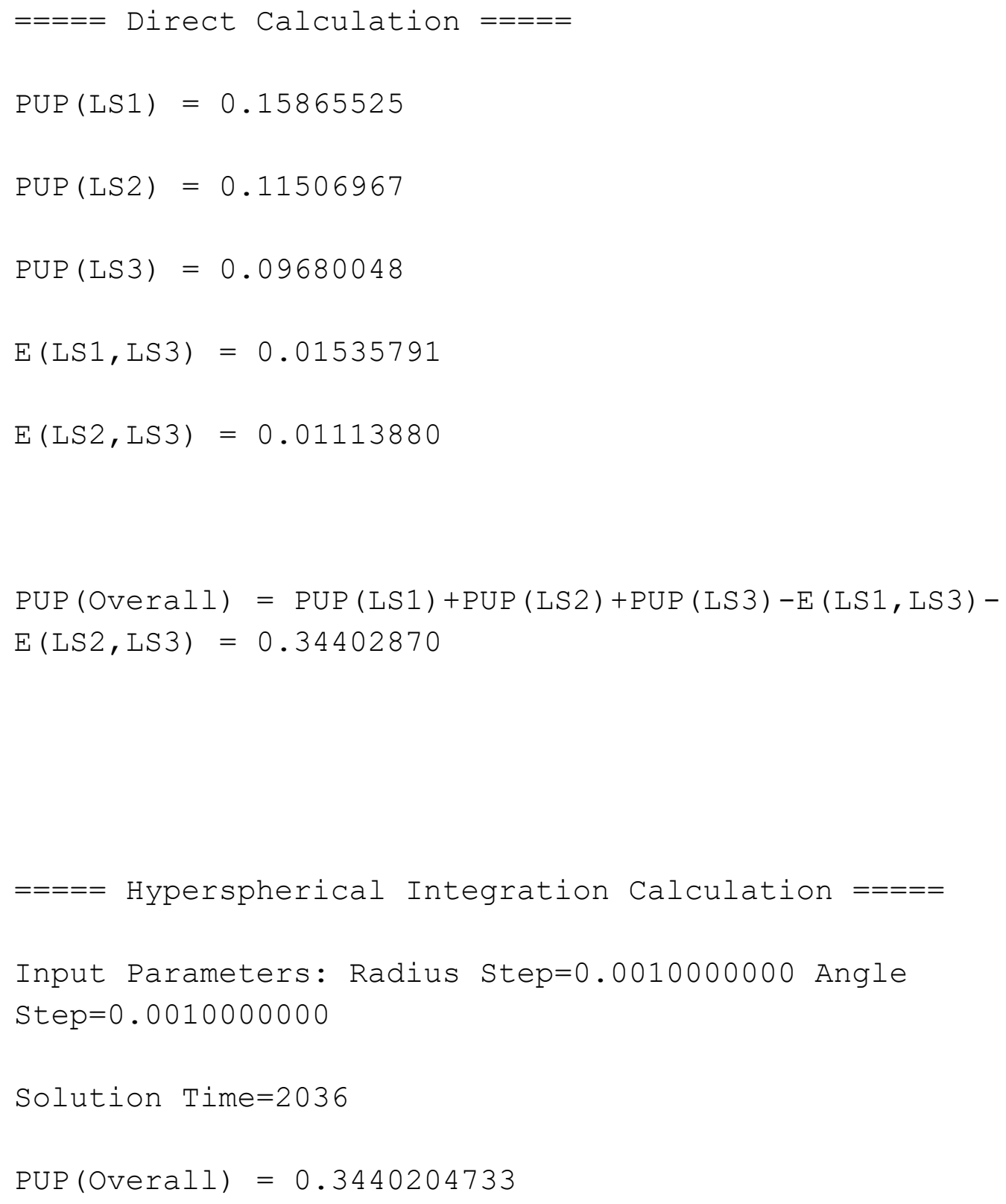


The second test case (Figure A-18) was designed to test the Ang and Tang (1974) Bounds method with correlated limit states. In this case, a design point was chosen for three hyperplanar limit states. The design points were (0.5,1.1), (-0.6,0.9), and (-0.9, -0.1) for Limit States 1, 2, and 3, respectively. The unsatisfactory performance regions were in the half-planes for the limit state response surfaces away from the origin.

Figure A-18. The second test case for PUPestimation.

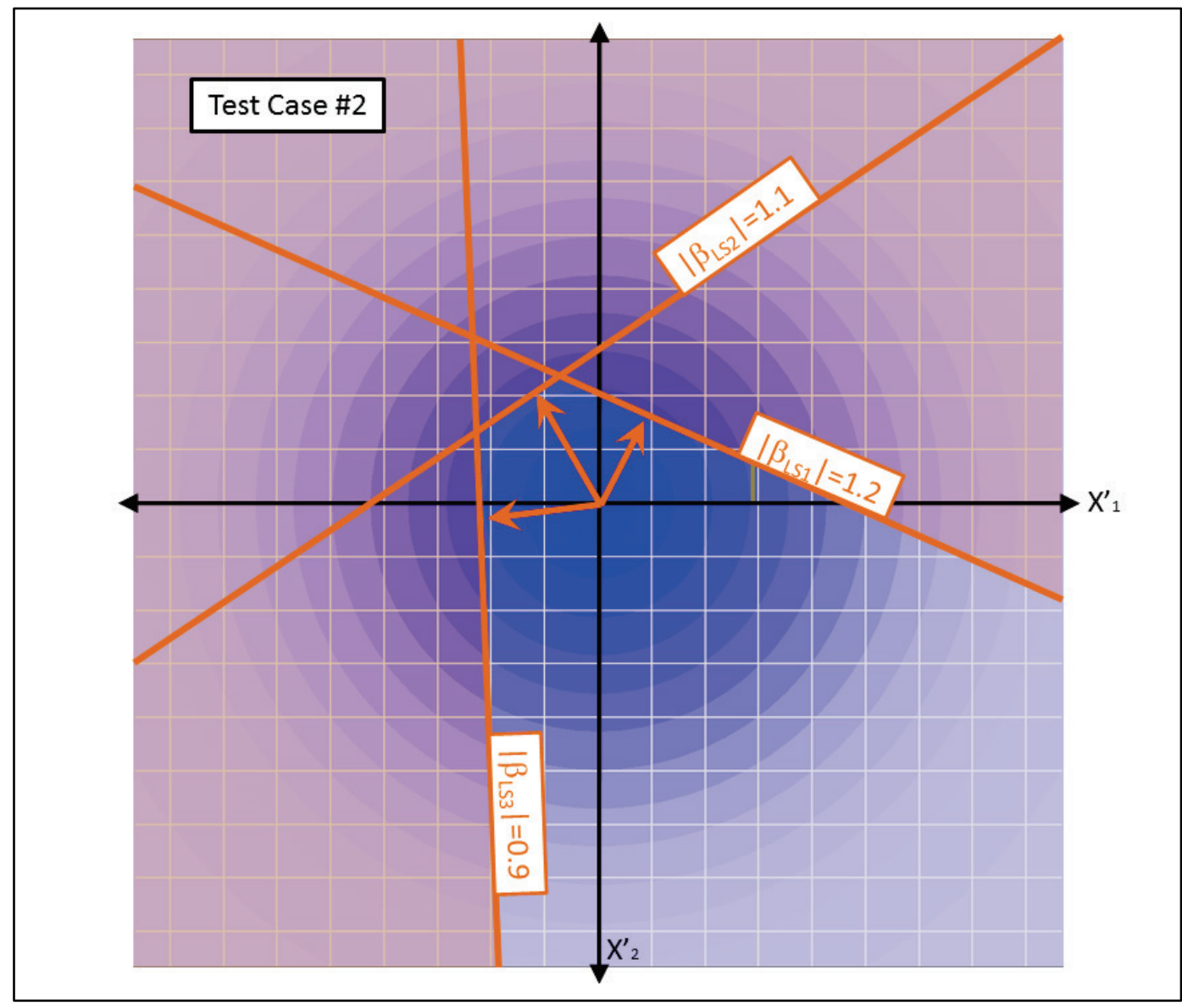

Listings A. 5 shows the input design points along with the calculated $\beta$ values and $\alpha$ vectors for the limit states. The absolute bounds for the PUP are calculated from Equations A.12 and A.13, resulting in $0.182590<=P U P<=0.376573$, with an average value of 0.279582 . Using the pairwise correlation relationships in Equations A.14 and A.15, with estimated values from Equations A.21-A.24, a tighter bound was established as $0.229335<=P U P<=0.344048$, with an average value of 0.286692 . Because of the correlation, an actual value was not available, so a polar integration was performed with a radius step of 0.01 and an angular step of 0.01, which resulted in a PUP of 0.329675 . 


\section{Listing A.5. Output from Ang and Tang (1974) Bounds estimation compared to polar} integration method.

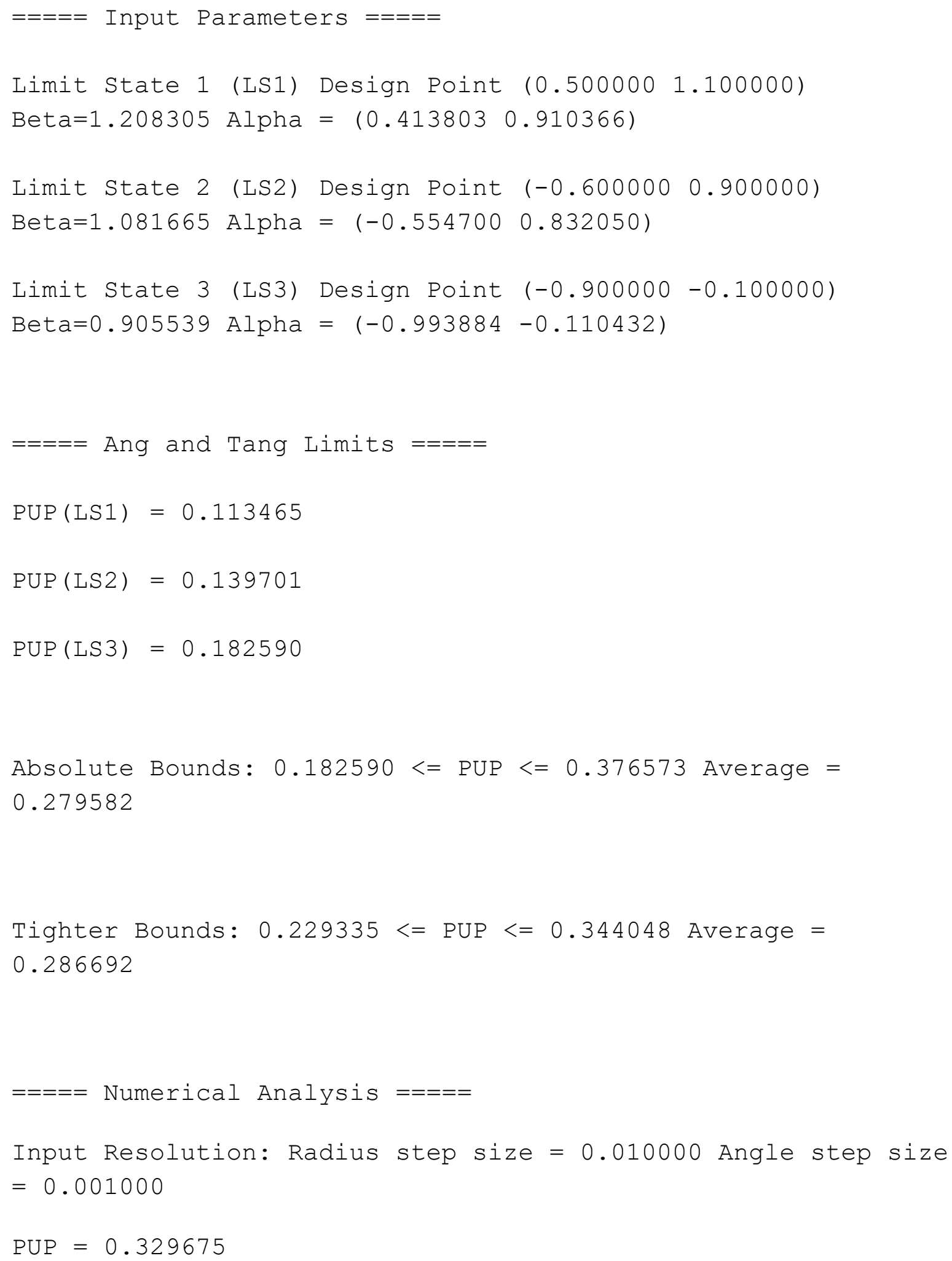


While the Bounds Estimation Method was simple to compute, the range of the values is too big for estimation in the tails of the Gaussian Normal distribution. The averaged values also were not accurate enough for the rigorous application that would be required by an LRFD solution method. 


\section{Appendix B: Earth and Water Pressure Distributions, Resultant Forces and Their Points of Application along the Faces of the Structural Wedge}

\section{B.1 Introduction: Resultant forces and their points of application along the two faces and the base of the Structural Wedge}

Figure B-1 shows the resultant left- and right-side earth forces $P_{L}$ and $P_{R}$, the left- and right-side resultant water forces $H_{L}$ and $H_{R}$ acting on the Structural Wedge, its base reaction force $N$, and base uplift force $U_{\text {base. }}$. Also included on this figure are the various distances to these forces and key geometry points.

Figure B-1. Free body diagram of forces acting on the Structural Wedge, including the left- and right-side forces resulting from the Resisting Wedge and Driving Wedge,

$P_{L}$ and $P_{R}$, respectively.

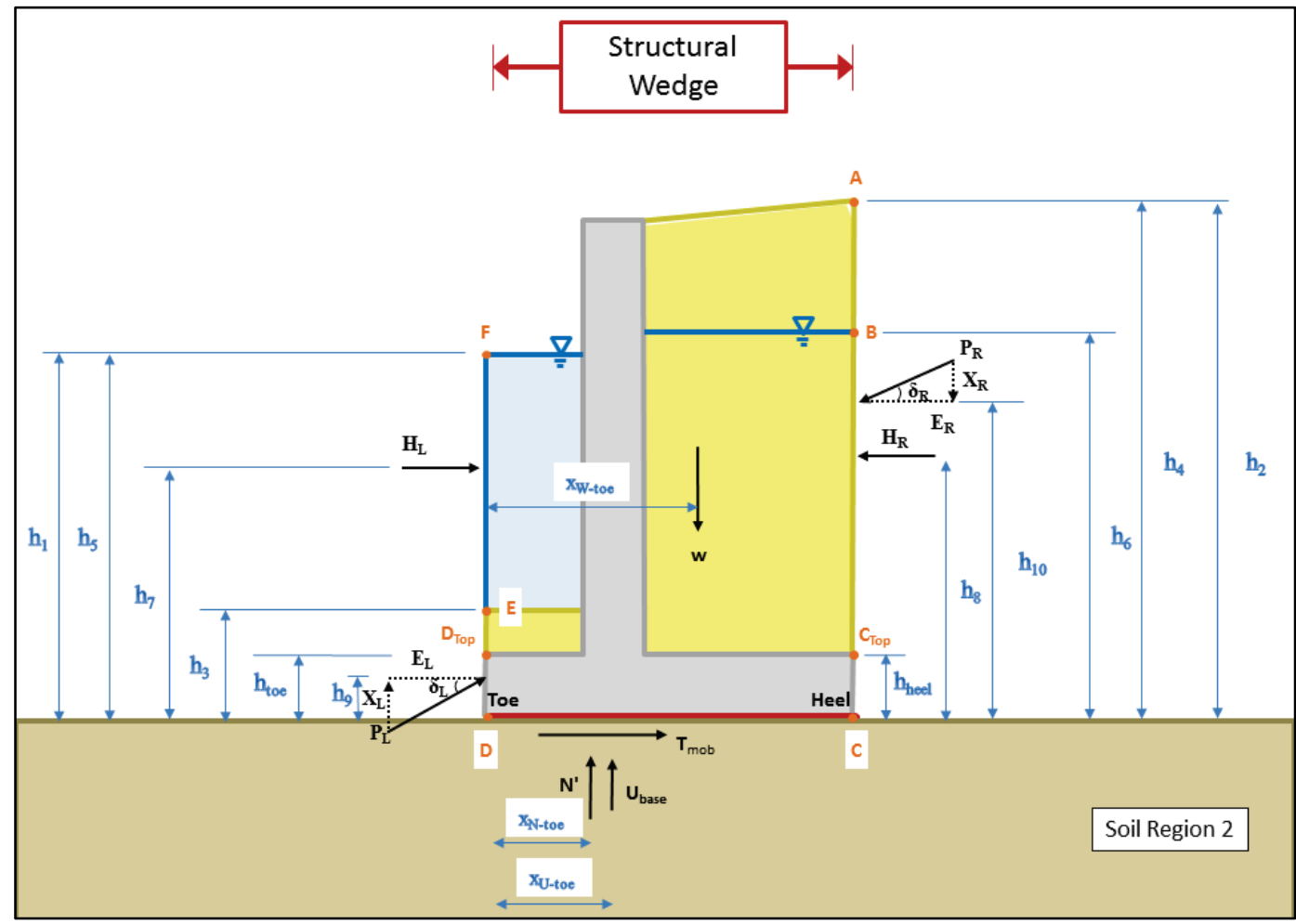




\section{B.2 Distribution of right-side interface earth pressures for a partially submerged, retained soil}

Figure B-2 shows the horizontal component of the effective earth pressure distribution and the distribution of water pressures with their corresponding resultant forces acting on the right side of the Structural Wedge.

Figure B-2. Horizontal component of effective earth pressures and water pressures with their corresponding resultant forces acting on the right side of the Structural Wedge.

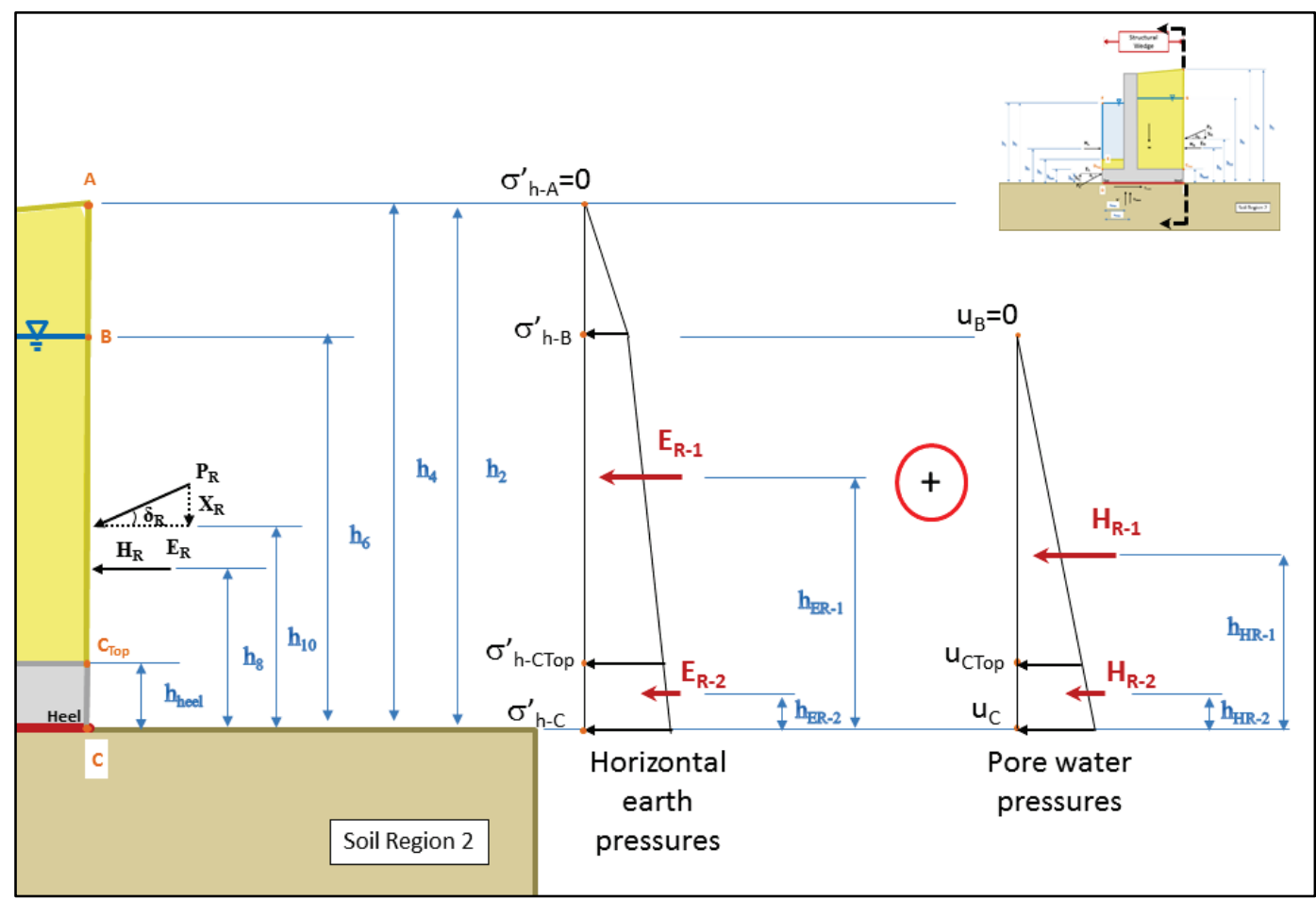

\section{B.2.1 Horizontal effective earth pressures - right side of the Structural Wedge}

The horizontal effective earth pressure at point B in Figure B-2 is given by

$$
\sigma_{h-B}^{\prime}=K_{\text {Active }} * \cos \left(\delta_{\text {mob-Active }}^{\prime}\right) * \gamma_{\text {moist }} *\left(h_{4}-h_{6}\right)
$$

where

$\gamma /$ moist is the moist unit weight of the retained soil and $K_{\text {Active }}$ is mobilized active earth pressure coefficient given by Equation 2.4 and is calculated using $\phi_{\text {mob-Active }}^{\prime}$ and $\boldsymbol{\delta}_{\text {mob-Active. }}$. 
The horizontal effective earth pressure at point $C_{T o p}$ is

$$
\sigma_{h-\text { CTop }}^{\prime}=\left\{\begin{array}{c}
K_{\text {Active }} * \cos \left(\delta^{\prime}{ }_{\text {mob-Active }}\right) * \\
\left\{\gamma_{\text {moist }} *\left(h_{4}-h_{6}\right)+\gamma_{1} *\left(h_{6}-h_{\text {heel }}\right)\right\}
\end{array}\right\}
$$

where

$\gamma_{1}$ is the effective unit weight of the retained soil accounting for the downward, vertical gradient of steady-state seepage through the saturated soil volume of the Driving Wedge. Equation 2.9 provides for the $\gamma_{1}$ value.

The horizontal effective earth pressure at point $\mathrm{C}$ is

$$
\sigma_{h-C}^{\prime}=\left\{\begin{array}{c}
K_{\text {Active }} * \cos \left(\delta^{\prime}{ }_{\text {mob-Active }}\right) * \\
\left\{\gamma_{\text {moist }} *\left(h_{4}-h_{6}\right)+\gamma_{1} * h_{6}\right\}
\end{array}\right\}
$$

\section{B.2.2 Pore water pressures - along the perimeter of the Structural Wedge}

The pore water pressures at submerged points B, CTop, C, D, and DTop of Figure B-1 are computed using the Line of Seepage procedure outlined in Subsection 2.3.1 for no gap or that in Subsection 2.3.2 with a gap extending from the heel of the Structural Wedge, along its base. The total heads $H_{A}$ at point $\mathrm{A}$ and $H_{E}$ at point $\mathrm{E}$ are the necessary boundary conditions to solve for the total heads at all of these interior points, as shown in the Transformed Line of Seepage plots of Figure 2-6.a (or Figure 2-8.a). Recall the pressure head $h_{p}$ is computed at any point along each of the three Figure 2-6 streamline segments using Bernoulli's equation for total head $(H)$ (and assuming the velocity head is negligible).

$$
h_{p}=H-h_{e}
$$

where $h_{e}$ is the elevation head for the point (from Figure 2-5). This modified relationship allows for the computation of the corresponding pore water pressure $(\mathrm{u})$ at the selected point by

$$
u=h_{p} * \gamma_{w}
$$

\section{B.2.3 Horizontal effective earth pressure resultant forces - right side of the Structural Wedge}

The resultant horizontal effective earth pressure force $E_{R-1}$ is given by 


$$
E_{R-1}=\frac{1}{2} *\left(\sigma_{h-B}^{\prime}\right) *\left(h_{4}-h_{6}\right)+\frac{1}{2} *\left(\sigma_{h-B}^{\prime}+\sigma_{h-C T o p}^{\prime}\right) *\left(h_{6}-h_{h e e l}\right)
$$

The point of application of $E_{R-1}$ is given by

$$
h_{E R-1}=\frac{\operatorname{MomER1_{A-B}+MomER1_{B-CTop}}}{E_{R-1}}
$$

with

$$
\operatorname{MomER} 1_{A-B}=\left[\frac{1}{2} *\left(\sigma_{h-B}^{\prime}\right) *\left(h_{4}-h_{6}\right)\right] *\left[\frac{1}{3} *\left(h_{4}-h_{6}\right)+h_{6}\right]
$$

and

$$
\left.\operatorname{MomER} 1_{B-C T o p}=\left\{\begin{array}{c}
\left\{\begin{array}{c}
{\left[\left(\sigma_{h-B}^{\prime}\right) *\left(h_{6}-h_{\text {heel }}\right)\right] *} \\
{\left[\frac{1}{2} *\left(h_{6}-h_{\text {heel }}\right)+h_{\text {heel }}\right]}
\end{array}\right\}+ \\
\left\{\left[\frac{1}{2} *\left(\sigma_{h-C T o p}^{\prime}-\sigma_{h-B}^{\prime}\right) *\left(h_{6}-h_{\text {heel }}\right)\right] *\right. \\
{\left[\frac{1}{3} *\left(h_{6}-h_{\text {heel }}\right)+h_{\text {heel }}\right]}
\end{array}\right\}\right\}
$$

The resultant horizontal effective earth pressure force $E_{R-2}$ is given by

$$
E_{R-2}=\frac{1}{2} *\left(\sigma_{h-C T o p}^{\prime}+\sigma_{h-c}^{\prime}\right) *\left(h_{\text {heel }}\right)
$$

The point of application for $E_{R-2}$ is given by

$$
h_{E R-2}=\frac{M o m E R 2 C T o p-C}{E_{R-2}}
$$

with

MomER2 $2_{\text {CTop }-C}=\left\{\left[\left(\sigma_{h-\text { CTop }}^{\prime}\right) *\left(h_{\text {heel }}\right)\right] *\left[\frac{1}{2} *\left(h_{\text {heel }}\right)\right]\right\}+\left\{\left[\frac{1}{2} *\left(\sigma_{h-C}^{\prime}-\sigma_{h-\text { CTop }}^{\prime}\right) *\left(h_{\text {heel }}\right)\right] *\right.$ $\left.\left[\frac{1}{3} *\left(h_{\text {heel }}\right)\right]\right\}$

The resultant horizontal effective earth pressure force $E_{R}$ is

$$
E_{R}=E_{R-1}+E_{R-2}
$$

and its point of application given by

$$
h_{10}=\frac{E_{R-1} * h_{E R-1}+E_{R-2} * h_{E R-2}}{E_{R}}
$$


The resultant mobilized effective, active earth pressure force $P_{R}$ is

$$
P_{R}=\frac{E_{R}}{\cos (\delta)}
$$

and its vertical force component $X_{R}$ is

$$
X_{R}=E_{R} * \tan (\delta)
$$

\section{B.2.4 Horizontal water pressure resultant forces - right side of the Structural Wedge}

The resultant pore water pressure force $H_{R-1}$ is given by

$$
H_{R-1}=\frac{1}{2} *\left(u_{C T o p}\right) *\left(h_{6}-h_{\text {heel }}\right)
$$

The point of application of $H_{R-1}$ with reference to the elevation of the heel, is given by

$$
h_{H R-1}=\frac{M o m H R 1_{B-C T o p}}{H_{R-1}}
$$

with the moment about the elevation of the bottom of the base of the slab, defined as

$$
\operatorname{MomHR}_{\text {B-CTop }}=\left\{\begin{array}{c}
{\left[\frac{1}{2} *\left(u_{\text {CTор }}\right) *\left(h_{6}-h_{\text {heel }}\right)\right] *} \\
{\left[\frac{1}{3} *\left(h_{6}-h_{\text {heel }}\right)+h_{\text {heel }}\right]}
\end{array}\right\}
$$

And the point of application of $H_{R-1}$ with reference to the elevation of top of base slab, corner point $\mathrm{C}_{\mathrm{Top}}$, is given by

$$
\left(h_{H R-1}-h_{\text {heel }}\right)=\frac{1}{3} *\left(h_{6}-h_{\text {heel }}\right)
$$

The resultant pore water pressure force $H_{R-2}$ is given by

$$
H_{R-2}=\frac{1}{2} *\left(u_{C T o p}+u_{c}\right) *\left(h_{\text {heel }}\right)
$$

The point of application for $H_{R-2}$ with reference to the elevation of the heel, is given by

$$
h_{H R-2}=\frac{M o m H R 2 C T o p-C}{H_{R-2}}
$$


with the moment about the elevation of the bottom of the base of the slab, defined as

$$
\left.M o m H R 2_{\text {CTop }-C}=\left\{\begin{array}{c}
\left\{\begin{array}{c}
{\left[\left(u_{\text {CTop }}\right) *\left(h_{\text {heel }}\right)\right] *} \\
{\left[\frac{1}{2} *\left(h_{\text {heel }}\right)\right]}
\end{array}\right\}+ \\
\left\{\left[\frac{1}{2} *\left(u_{\text {CTop }}-u_{C}\right) *\left(h_{\text {heel }}\right)\right] *\right. \\
{\left[\frac{1}{3} *\left(h_{\text {heel }}\right)\right]}
\end{array}\right\}\right\}
$$

\section{B.3 Resultant forces acting on the soil block - right, located above the heel portion of the base slab for the Structural Wedge}

Figure B-3 shows the resultant earth pressure and water pressure resultant forces acting on the soil block located above the heel portion of the base slab for the Structural Wedge. It is designated "soil block - right" because this soil block is situated to the right of the reinforced concrete stem. The stem as well as the reinforced concrete base slab bound the left and bottom faces of this soil block. Observe that no horizontal shear force $(T)$ is assumed to act along the base of soil block - right, consistent with the EM 1110-2-2502 (HQUSACE 1989) assumption.

Figure B-3. Free body diagram of resultant forces acting on and within the soil block (right) located above the heel portion of the base slab for the Structural Wedge.

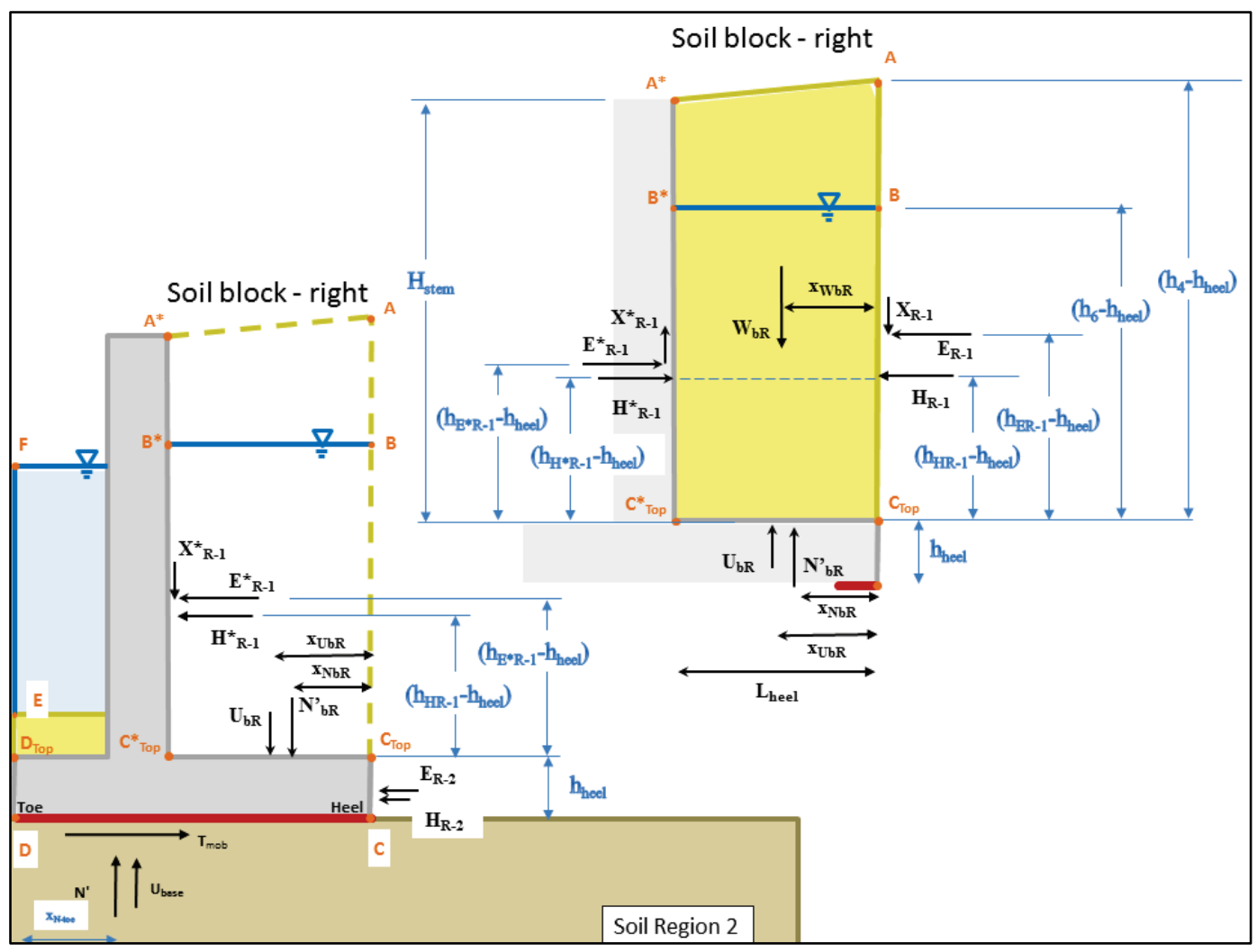


Values for forces $E_{R-1}, X_{R-1}$, and $H_{R-1}$ are computed from the earth and water pressure distributions along the imaginary vertical section extending up from the heel of the T-Wall through the retained soil using the equations given in Subsections B.2.3 and B.2.4, respectively. Recall there is no horizontal shear force $(T)$ assumed to act along the base of soil block right, consistent with the EM 1110-2-2502 (HQUSACE 1989) assumption. Then horizontal force equilibrium applied to the Figure B-3 soil block right, the resultant horizontal effective earth pressure force $E^{*}{ }_{R-1}$ applied to the stem is defined as

$$
E^{*}{ }_{R-1}=E_{R-1}+H_{R-1}-H^{*}{ }_{R-1}
$$

For the constant elevation piezometric surface in the partially submerged retained soil block - right of Figure B-3 with a constant, vertical (downward) seepage gradient,

$$
H *_{R-1}=H_{R-1}
$$

and the $H_{R-1}^{*}$ point of action will be the same as that for $H_{R-1}$ (Equation B.16),

$$
\left(h_{H^{*} R-1}-h_{\text {heel }}\right)=\left(h_{H R-1}-h_{\text {heel }}\right)=\frac{1}{3} *\left(h_{6}-h_{\text {heel }}\right)
$$

Consequently, by Equation B.20,

$$
E_{R-1}^{*}=E_{R-1}
$$

For this T-Wall geometry, $h_{E^{*} R-1}$ was set equal to $h_{E R-1}$ because a complete solution for the location of this force is a complex computation that is not anticipated to provide for a significant change from this assumption.

Quantitative assessment of the vertical shear force $X^{*}{ }_{R-1}$ exerted on the stem by the soil block - right is not as straightforward as one might hope. There are significant SSI issues that influence the interface forces within the soil block - right of the Structural Wedge. For example, a right triangle-shaped soil region adjacent to the vertical interface between the roughened surface of the reinforced concrete stem face (with the triangle's base defined by the base slab) is not contained within the inverted triangular volume of soil possessing a fully mobilized shear strength (Figure B-4). Also depicted in Figure B-4 is the inverted triangular volume of slip planes centered with its 
tip at the heel of the T-Wall. This is the active earth pressure soil zone, contained within this inverted triangular volume feature, that has its soil shear strength mobilized to the level of $\phi^{\prime}$ mob-Active. Therefore, if $F S_{\text {Active }}$ equals 1.0, then $\phi^{\prime}$ mob-Active is equal to $\phi$ ' of soil region 1. This inverted triangular soil volume forms as the Structural Wedge moves away from the Driving Soil Wedge, with this inverted triangular soil volume dropping downward as the T-Wall moves out. A graben will form above this inverted triangular soil volume due to this downward soil mass movement.

Figure B-4. Active and passive Rankine states of stress behind and in front of a cantilever retaining wall, respectively (after Peck et al. 1974).

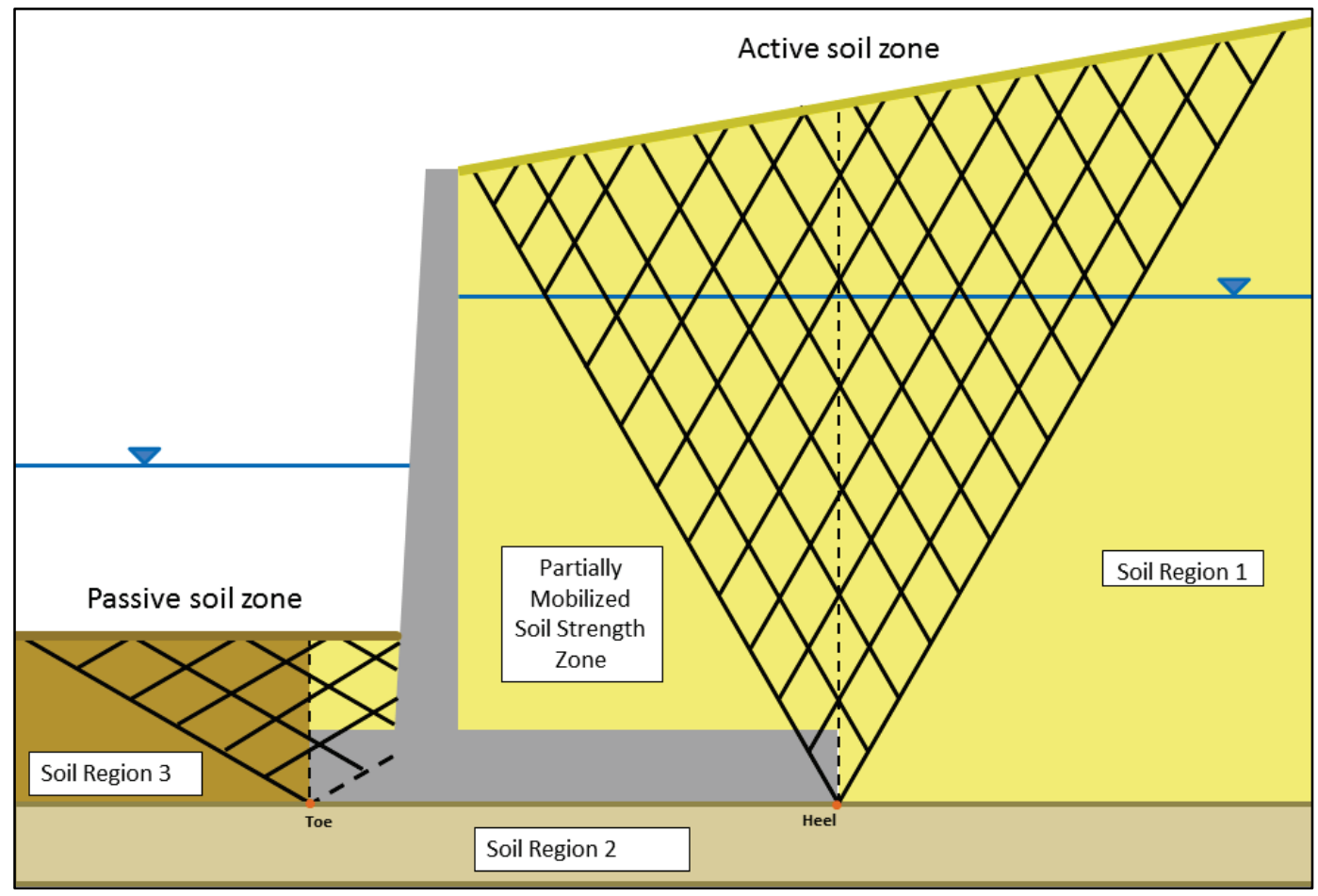

Because the reinforced concrete stem face is located outside of the inverted triangular soil volume, the zone of soil adjacent to the vertical stem face interfaces does not mobilize its shear strength as a result of lateral T-Wall movement and active soil wedge development in the soil zone above the heel of the T-Wall. Thus, there is a right-angled triangular soil pocket that does not fully mobilize its shear strength (Figure B-4). It is situated between the stem face and the left side of the soil contained within the Figure B-4 inverted triangle of retained soil (active earth pressure zone) and above the top of the base slab.

Recall this Figure B-4 soil regime of fully mobilized shear strength above the heel of the T-Wall takes the geometric form of an inverted, triangular 
volume of soil. The orientation of the bottom-most slip plane defining the right side of this inverted triangle into the soil of the Driving Wedge is oriented from horizontal by the angle given by Equation 2.11. For a compacted, cohesionless engineered backfill consisting of granular soil, this angle is equal to approximately $60 \mathrm{deg}$ from horizontal. This inverted, triangular volume of soil also extends into the soil of the soil block - right within the Structural Wedge, as shown in Figure B-4. The lateral extent of the active soil regime into soil block - right is approximated as being defined geometrically by a (slip) plane emanating from the heel of the T-Wall and oriented from horizontal at this same Equation 2.11 slip plane angle of approximately $60 \mathrm{deg}$ from horizontal. For a heel base slab that is $18 \mathrm{ft}$ wide, as measured from stem to heel and with a $26.5 \mathrm{ft}$ tall stem over a $3.5 \mathrm{ft}$ thick base slab, the right angled triangular soil zone next to the stem face for soil block - right is outside of the Figure B-4 triangular active wedge soil zone. Thus, active soil wedge theory would not be applied to determine the value for the vertical shear force $X_{R-1}^{*}$ exerted on the stem by the rightangled triangular soil pocket.

The approach taken to compute a vertical shear force $X_{R-1}^{*}$ is a complete SSI-based approach developed through research first investigated by Dr. Ebeling (Ebeling 1989). This type of shear force is named vertical shear and is not associated with the development of the active soil wedge and its inverted, triangular volume of soil (Figure B-4). The vertical shear force $X^{*}{ }_{R-1}$ exerted on the stem by the soil block - right is calculated using the Vertical Shear Force computational procedure outlined in Appendix $\mathrm{F}$ procedure of EM 1110-2-2100 (HQUSACE 2005). This vertical shear force results from the differential settlement of the soil during backfilling of the T-Wall after its construction. This differential settlement and resulting vertical shear force arise due to the soil hanging on the roughened concrete vertical (stem) surface. Recall that this soil region adjacent to the vertical soil-structure interface is beyond the active soil wedge zone that develops with a focal point of the heel of the T-Wall and as a result of the movement of the T-Wall away from its retained soil.

$$
\left.X^{*}{ }_{R-1}=\left\{\begin{array}{c}
\left(K * C_{w t}\right) * \\
\left\{\left[\frac{1}{2} * \gamma_{\text {moist }} *\left(H_{\text {stem }}-\left(h_{6}-h_{\text {heel }}\right)\right)^{2}\right]+\right. \\
{\left[\gamma_{\text {moist }} *\left(H_{\text {stem }}-\left(h_{6}-h_{\text {heel }}\right)\right) *\left(h_{6}-h_{\text {heel }}\right)\right]+} \\
{\left[\frac{1}{2} * \gamma_{1} *\left(h_{6}-h_{\text {heel }}\right)^{2}\right]}
\end{array}\right\}\right\}
$$


with $K$ equal to 0.1 by EM 1110-2-2100 (HQUSACE 2005) Figure F-3 for a stem wall of height equal to $26.5 \mathrm{ft}\left(\mathrm{H}_{\text {stem }}\right)$, as measured form the top of base slab, and retaining dense, compacted granular fill. The correction factor $C_{w t}$ for post-backfilling submergence of the compacted backfill is given by

$$
C_{w t}=\left\{1-\frac{\left(h_{4}-h_{6}\right)}{H_{\text {stem }}}\right\}
$$

For $\left(h_{4}-h_{6}\right)$ equal to $6 \mathrm{ft}$ and $H_{\text {stem }}$ equal to $26.5 \mathrm{ft}, C_{w t}$ becomes 0.23 . Thus, the term $\left(K^{*} C_{w t}\right)$ is equal to $0.023\left(=0.1^{*} 0.23\right)$. If the compacted, dense granular backfill was not submerged after placement, the vertical shear coefficient would remain a value of 0.1 since the correction factor $C w t$ would then be equal to 1 .

Vertical force equilibrium applied to the Figure B-3 soil block - right results in the effective force $N^{\prime} b R$ normal to the base of soil block - right being defined as

$$
N_{b R}^{\prime}=W_{b R}+X_{R-1}+-X_{R-1}^{*}-U_{b R}
$$

The vertical force $X_{R-1}$ is

$$
X_{R-1}=E_{R-1} * \tan \left(\delta^{\prime}{ }_{\text {mob-Active }}\right)
$$

The weight of soil block - right is given by

$$
W_{b R}=\left\{\begin{array}{c}
\gamma_{\text {moist }} *\left[\frac{1}{2} *\left(\left(h_{4}-h_{\text {heel }}\right)-H_{\text {stem }}\right) * L_{\text {heel }}\right]+ \\
\gamma_{\text {moist }} *\left[\left(H_{\text {stem }}-\left(h_{6}-h_{\text {heel }}\right)\right) * L_{\text {heel }}\right]+ \\
\gamma_{\text {saturated }} *\left[\left(h_{6}-h_{\text {heel }}\right) * L_{\text {heel }}\right]
\end{array}\right\}
$$

Its positon $x_{W b R}$ relative to the heel of the base slab is

$$
x_{W b R}=\frac{\left\{\begin{array}{c}
\gamma_{\text {moist }} *\left[\frac{1}{2} *\left(\left(h_{4}-h_{\text {heel }}\right)-H_{\text {stem }}\right) * L_{\text {heel }}\right] *\left[\frac{1}{3} * L_{\text {heel }}\right]+ \\
\gamma_{\text {moist }} *\left[\left(H_{\text {stem }}-\left(h_{6}-h_{\text {heel }}\right)\right) * L_{\text {heel }}\right] *\left[\frac{1}{2} * L_{\text {heel }}\right]+ \\
\gamma_{\text {saturated }} *\left[\left(h_{6}-h_{\text {heel }}\right) * L_{\text {heel }}\right] *\left[\frac{1}{2} * L_{\text {heel }}\right]
\end{array}\right\}}{W_{\text {bR }}}
$$

With a horizontal phreatic surface within soil block - right, the uplift water pressure acting normal to the base of this soil block will be uniform. With a 
uniform downward acting seepage gradient $i_{y-D W}$ within the soil block-right, the resultant uplift force $U_{b R}$ is

$$
U_{b R}=\left[\gamma_{\text {water }} *\left(1-i_{y-D W}\right) *\left(h_{6}-h_{\text {heel }}\right)\right] * L_{\text {heel }}
$$

Its positon $x_{U b R}$ relative to the outside, top corner of the base slab, point CTop, is

$$
x_{U b R}=L_{\text {heel }} / 2
$$

Moment equilibrium applied to the Figure B-3 soil block - right results in the position of the effective force $N^{\prime}{ }_{b R}$ normal to the base of soil block right, as measured from the outside, top corner of the base slab, point $\mathrm{C}_{\mathrm{Top}}$, defined as

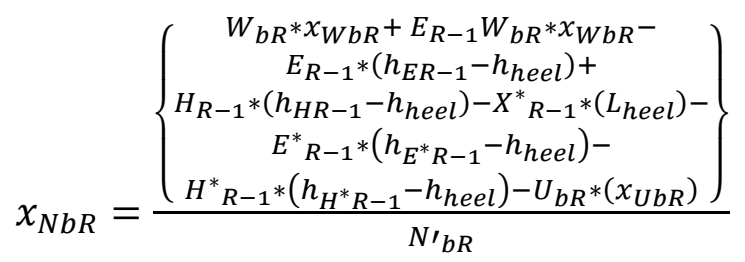

The horizontal effective earth pressure force $E_{R-1}^{*}$ acts on the stem at a height above the base slab equal to

$$
h_{E^{*} R-1}=\frac{\left\{\begin{array}{c}
{\left[\frac{1}{2} * \gamma_{\text {moist }} *\left(H_{\text {stem }}-\left(h_{6}-h_{\text {heel }}\right)\right)^{2}\right] *\left[\frac{1}{3} *\left(H_{\text {stem }}-\left(h_{6}-h_{\text {heel }}\right)\right)+\left(h_{6}-h_{\text {heel }}\right)\right]+} \\
{\left[\gamma_{\text {moist }} *\left(H_{\text {stem }}-\left(h_{6}-h_{\text {heel }}\right)\right) *\left(h_{6}-h_{\text {heel }}\right)\right] *\left[\frac{1}{2} *\left(h_{6}-h_{\text {heel }}\right)\right]+} \\
{\left[\frac{1}{2} * \gamma_{1} *\left(h_{6}-h_{\text {heel }}\right)^{2}\right] *\left[\frac{1}{3} *\left(h_{6}-h_{\text {heel }}\right)\right]}
\end{array}\right\}}{\left\{\begin{array}{c}
{\left[\frac{1}{2} * \gamma_{\text {moist }} *\left(H_{\text {stem }}-\left(h_{6}-h_{\text {heel }}\right)\right)^{2}\right]+} \\
{\left[\gamma_{\text {moist }} *\left(H_{\text {stem }}-\left(h_{6}-h_{\text {heel }}\right)\right) *\left(h_{6}-h_{\text {heel }}\right)\right]+} \\
{\left[\frac{1}{2} * \gamma_{1} *\left(h_{6}-h_{\text {heel }}\right)^{2}\right]}
\end{array}\right\}}(\mathrm{B}
$$

with $\gamma_{1}$ given by Equation 2.9 for a constant vertical, downward seepage.

The horizontal water pressure force $H^{*}{ }_{R-1}$ acts normal to the stem and at a height above the top of the base slab equal to

$$
\left(h_{H^{*} R-1}-h_{\text {heel }}\right)=\frac{1}{3} *\left(h_{6}-h_{\text {heel }}\right)
$$

\section{B.4 Distribution of left-side interface earth pressures for a partially}




\section{submerged, retained soil}

Figure B-5 shows the horizontal component of the effective earth pressure distribution and the distribution of water pressures with their corresponding resultant forces acting on the left side of the Structural Wedge.

Figure B-5. Horizontal component of effective earth pressures and water pressures with their corresponding resultant forces acting on the left side of the Structural Wedge.

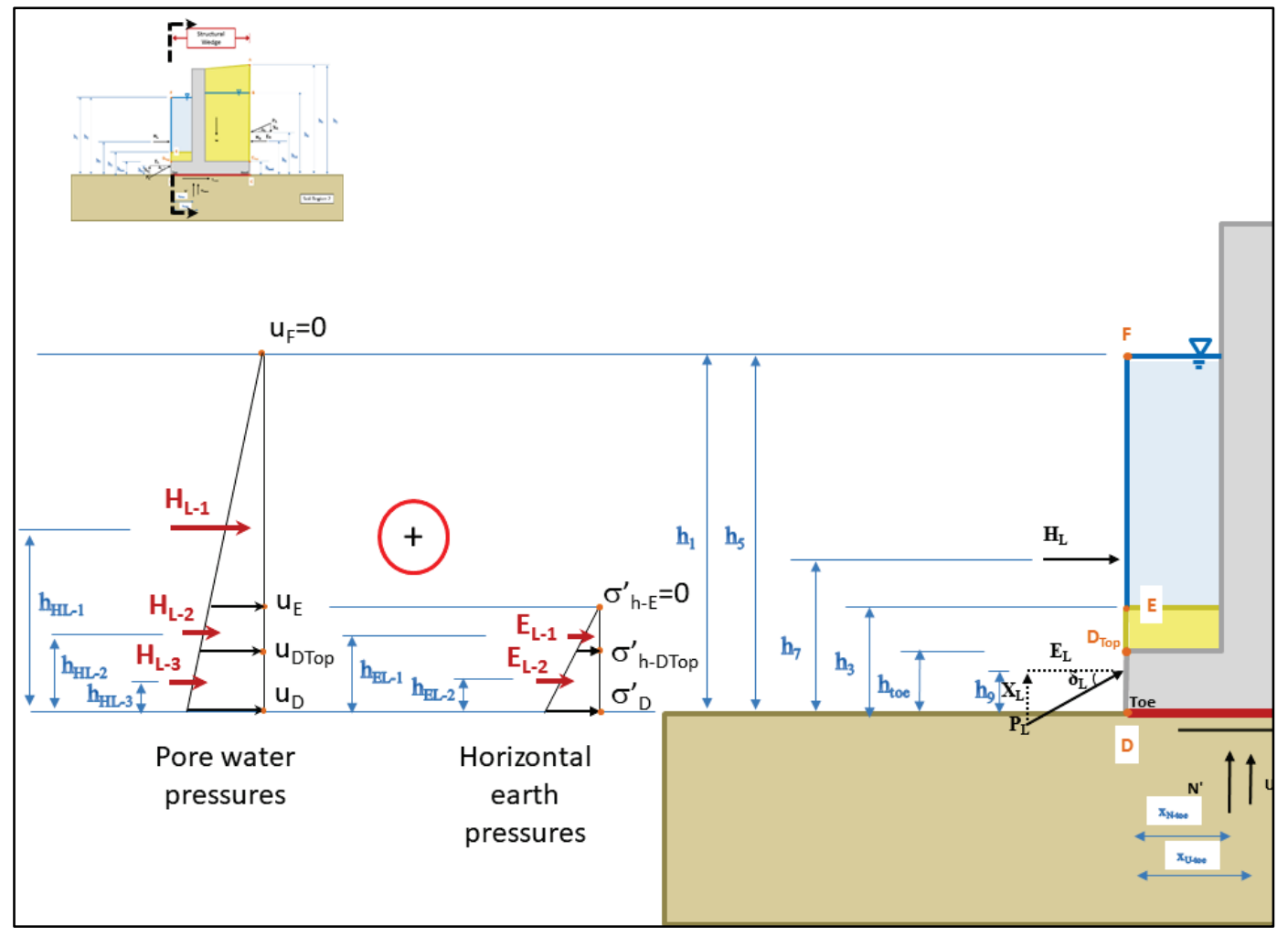

\section{B.4.1 Horizontal effective earth pressures - left side of the Structural Wedge}

The horizontal effective earth pressure at point E in Figure B-5 is zero. The horizontal effective earth pressure at point $\mathrm{D}_{\text {Top }}$ is given by

$$
\sigma_{h-D T o p}^{\prime}=K_{\text {Passive }} * \cos \left(\delta_{\text {mob-Passive }}^{\prime}\right) * \gamma_{1} *\left(h_{3}-h_{\text {toe }}\right)
$$

where $\gamma_{1}$ is the effective unit weight of the retained soil accounting for the upward, vertical gradient of steady-state seepage through the saturated soil volume of the Driving Wedge. Equation 2.19 provides for the $\gamma_{1}$ value. 
K Passive is mobilized passive earth pressure coefficient given by Equation 2.16 and is calculated using $\phi^{\prime}$ mob-Active and $\delta_{\text {mob-Active. }}^{\prime}$

The horizontal effective earth pressure at point $\mathrm{D}$ is

$$
\sigma_{h-D}^{\prime}=K_{\text {Passive }} * \cos \left(\delta_{\text {mob-Passive }}^{\prime}\right) *\left\{\gamma_{1} * h_{3}\right\}
$$

\section{B.4.2 Horizontal effective earth pressure resultant forces - left side of the Structural Wedge}

The resultant horizontal effective earth pressure force $E_{L-1}$ is given by

$$
E_{L-1}=\frac{1}{2} *\left(\sigma_{h-D T o p}^{\prime}\right) *\left(h_{3}-h_{\text {toe }}\right)
$$

The point of application of $E_{L-1}$, relative to the bottom of the base slab, is given by

$$
h_{E L-1}=\frac{M o m E L 1_{D T o p-E}}{E_{L-1}}
$$

with

$$
\operatorname{MomEL} 1_{\text {DTop-E }}=\left\{\begin{array}{c}
{\left[\frac{1}{2} *\left(\sigma_{h-D T o p}^{\prime}\right) *\left(h_{3}-h_{\text {toe }}\right)\right] *} \\
{\left[\frac{1}{3} *\left(h_{3}-h_{\text {toe }}\right)+h_{\text {toe }}\right]}
\end{array}\right\}
$$

The resultant horizontal effective earth pressure force $E_{L-2}$ is given by

$$
E_{L-2}=\frac{1}{2} *\left(\sigma_{h-D T o p}^{\prime}+\sigma_{D}^{\prime}\right) *\left(h_{\text {toe }}\right)
$$

The point of application for $E_{L-2}$, relative to the bottom of the base slab, is given by

$$
h_{E L-2}=\frac{M o m E L 2_{D-D T o p}}{E_{L-2}}
$$

with

$$
M o m E L 2_{D-D T o p}=\left\{\begin{array}{c}
\left\{\left[\left(\sigma_{h-D T o p}^{\prime}\right) *\left(h_{\text {toe }}\right)\right] *\left[\frac{1}{2} *\left(h_{\text {toe }}\right)\right]\right\}+ \\
\left\{\left[\frac{1}{2} *\left(\sigma_{h-D}^{\prime}-\sigma_{h-D T o p}^{\prime}\right) *\left(h_{\text {toe }}\right)\right] *\left[\frac{1}{3} *\left(h_{\text {toe }}\right)\right]\right\}
\end{array}\right\}(B
$$


The resultant horizontal effective earth pressure force $E_{R}$ is

$$
E_{L}=E_{L-1}+E_{L-2}
$$

and its point of application, relative to the bottom of the base slab, given by

$$
h_{9}=\frac{E_{L-1} * h_{E L-1}+E_{L-2} * h_{E L-2}}{E_{L}}
$$

The resultant effective earth pressure force $P_{L}$ is

$$
P_{L}=\frac{E_{L}}{\cos \left(\delta_{\text {mob-Passive }}^{\prime}\right)}
$$

and its vertical force component $X_{L}$ is

$$
X_{L}=E_{L} * \tan \left(\delta_{m o b-P a s s i v e}^{\prime}\right)
$$

\section{B.4.3 Horizontal water pressure resultant forces - left side of the Structural Wedge}

The computation of pore water pressures acting normal to the Structural Wedge using the Line of Seepage procedure of analysis is discussed in Subsection B.2.2. The resultant pore water pressure force $H_{L-1}$ is given by

$$
H_{L-1}=\frac{1}{2} *\left(u_{E}\right) *\left(h_{5}-h_{3}\right)
$$

The point of application of $H_{L-1}$, relative to the bottom of the base slab, is given by

$$
h_{H L-1}=\frac{M o m H L 1_{F-E}}{H_{L-1}}
$$

with

$$
\operatorname{MomHL} 1_{F-E}=\left[\frac{1}{2} *\left(u_{E}\right) *\left(h_{5}-h_{3}\right)\right] *\left[\frac{1}{3} *\left(h_{5}-h_{3}\right)+h_{3}\right]
$$

And the point of application of $H_{L-1}$ with reference to the elevation of top of base slab, corner point $D_{T o p}$, is given by 


$$
\left(h_{H L-1}-h_{t o e}\right)=\frac{1}{3} *\left(h_{5}-h_{t o e}\right)
$$

The resultant pore water pressure force $H_{L-2}$ is given by

$$
H_{L-2}=\frac{1}{2} *\left(u_{E}+u_{D T O p}\right) *\left(h_{3}-h_{\text {toe }}\right)
$$

The point of application for $H_{L-2}$, relative to the bottom of the base slab, is given by

$$
h_{H L-2}=\frac{M o m H L 2_{E-D T o p}}{H_{L-2}}
$$

with

$$
M o m H L 2_{E-D T O p}=\left\{\begin{array}{c}
\left\{\left[\left(u_{E}\right) *\left(h_{3}-h_{\text {toe }}\right)\right] *\right. \\
\left.\left[\frac{1}{2} *\left(h_{3}-h_{\text {toe }}\right)+h_{\text {toe }}\right]\right\} \\
\left\{\left[\begin{array}{c}
\left.\frac{1}{2} *\left(u_{D T o p}-u_{E}\right) *\left(h_{3}-h_{\text {toe }}\right)\right] * \\
{\left[\frac{1}{3} *\left(h_{3}-h_{\text {toe }}\right)+h_{\text {toe }}\right]}
\end{array}\right\}\right.
\end{array}\right\}
$$

The resultant pore water pressure force $H_{L-3}$ is given by

$$
H_{L-3}=\frac{1}{2} *\left(u_{D T o p}+u_{D}\right) *\left(h_{\text {toe }}\right)
$$

The point of application for $H_{L-3}$, relative to the bottom of the base slab, is given by

$$
h_{H L-3}=\frac{M o m H L 3_{D T o p-D}}{H_{L-3}}
$$

with

$$
\operatorname{MomHL}_{\text {DTop }-D}=\left\{\begin{array}{c}
\left\{\left[\left(u_{\text {DTop }}\right) *\left(h_{\text {toe }}\right)\right] *\left[\frac{1}{2} *\left(h_{\text {toe }}\right)\right]\right\}+ \\
\left\{\begin{array}{c}
{\left[\frac{1}{2} *\left(u_{D}-u_{D T o p}\right) *\left(h_{\text {toe }}\right)\right] *} \\
{\left[\frac{1}{3} *\left(h_{\text {toe }}\right)\right]}
\end{array}\right\}
\end{array}\right\}
$$

\section{B.5 Resultant forces acting on the soil block - right, located above the toe portion of the base slab for the Structural Wedge}

Figure B-6 shows the resultant earth pressure and water pressure resultant forces acting on the soil block located above the toe portion of the base slab for the Structural Wedge. Recall that the soil-based interface forces acting on 
the interface extending upwards from the toe of the T-Wall are a result of the Structural Wedge plowing into the Resisting Wedge. The Figure B-6 region of the Structural Wedge is designated "soil block - left" because this soil block is situated to the left of the reinforced concrete stem. The stem as well as the reinforced concrete base slab bound the right and bottom faces of this soil block. Observe that no horizontal shear force $(T)$ is assumed to act along the base of soil block - left, consistent with the EM 1110-2-2502 (HQUSACE 1989) assumption.

Figure B-6. Free body diagram of resultant forces acting on and within the soil block (left) located above the toe portion of the base slab for the Structural Wedge.

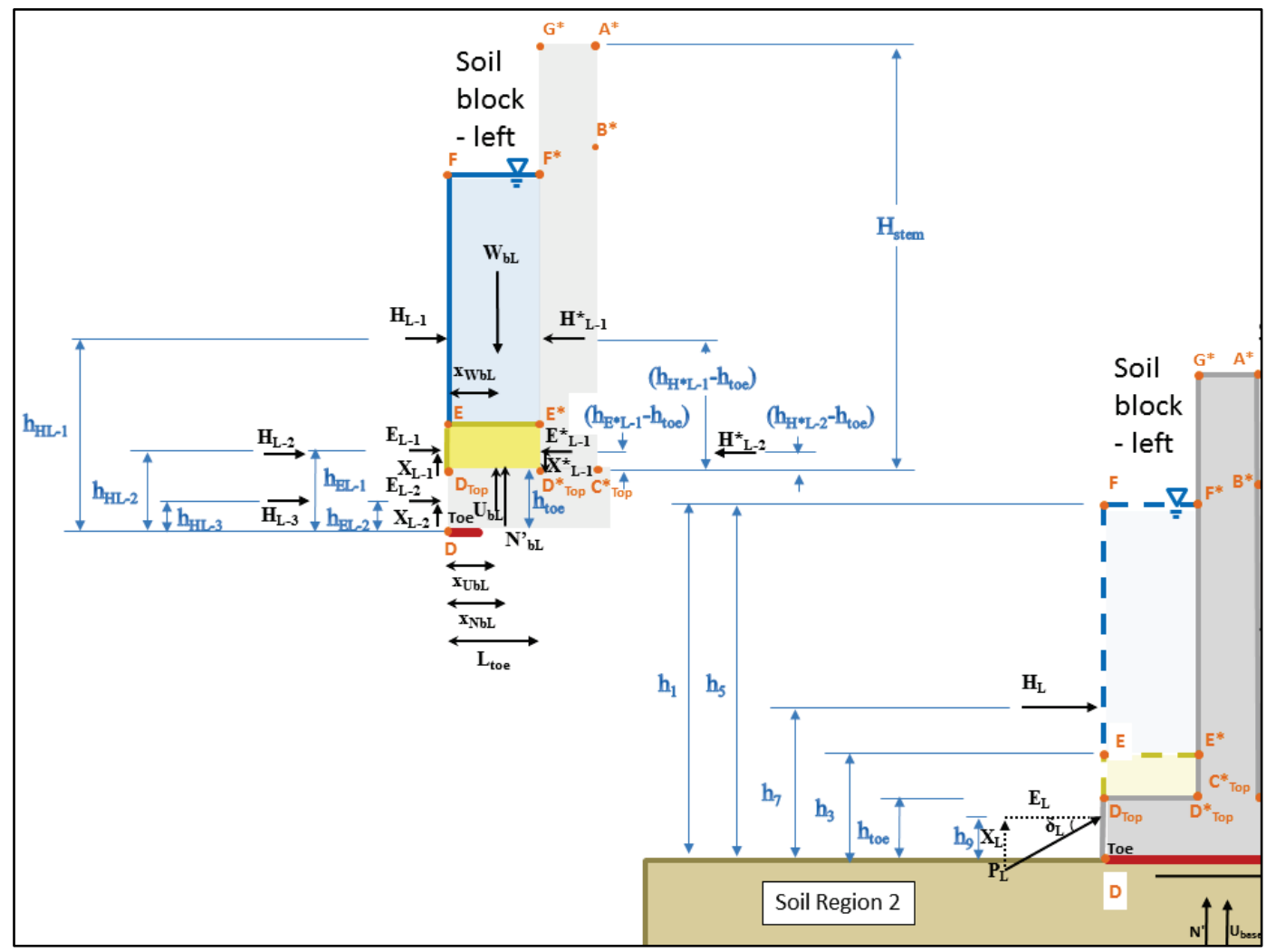

Values for forces $E_{L-1}, X_{L-1}$, and $H_{L-1}$ are computed from the earth and water pressure distributions along the imaginary vertical section extending up from the heel of the T-Wall through the retained soil using the equations given in Subsections B.4.2 and B.4.3, respectively. Recall there is no horizontal shear force $(T)$ assumed to act along the base of soil block - left, consistent with the EM 1110-2-2502 (HQUSACE 1989) assumption. Then, by horizontal force equilibrium applied to the Figure B-6 soil block - left, the resultant horizontal effective earth pressure force $E^{*}{ }_{L-1}$ applied to the stem is defined as 


$$
E_{L-1}^{*}=E_{L-1}+H_{L-1}-H^{*}{ }_{L-1}
$$

For the constant elevation piezometric surface in the partially submerged retained soil block - left of Figure B-6 with a constant vertical (upward) seepage gradient, the boundary water pressure distributions and their resultant forces are equivalent.

$$
\begin{aligned}
& H^{*}{ }_{L-1}=H_{L-1} \\
& H^{*}{ }_{L-2}=H_{L-2}
\end{aligned}
$$

Consequently, by Equation B.55,

$$
E_{L-1}^{*}=E_{L-1}
$$

This equivalency is judged appropriate for the level ground scenario above the base slab. By the same logic regarding the symmetrical pressure distributions,

$$
\begin{aligned}
& \left(h_{H^{*} L-1}-h_{\text {heel }}\right)=\left(h_{H L-1}-h_{\text {heel }}\right) \\
& \left(h_{H^{*} L-2}-h_{\text {heel }}\right)=\left(h_{H L-2}-h_{\text {heel }}\right) \\
& \left(h_{E^{*} L-1}-h_{\text {heel }}\right)=\left(h_{E L-1}-h_{\text {heel }}\right)
\end{aligned}
$$

For this T-Wall geometry, $h_{E^{*} L-1}$ was set equal to $h_{E L-1}$ because a complete solution for the location of this force is a complex computation that is not anticipated to provide for a significant change from this assumption.

Because of the geometric configuration of the soil region at the toe, the majority of soil block - left, located above the toe of the base slab will be contained within the passive soil wedge feature developing within the buttressing soil wedge zone (Figure B-4). Therefore, the vertical shear force computational procedure (Ebeling 1989) due to differential settlement along the roughened concrete stem that was used to compute $X_{R-1}^{*}$ of soil block - right, cannot be used to compute $X_{L-1}^{*}$.

Instead, the value of $X^{*} R-1$ will be computed based upon the assumption of a mobilized passive earth pressure zone of soil defined geometrically by an inverted triangular soil volume that encroaches into soil block - right of the 
Structural Wedge as well as into the Resisting Wedge. This inverted triangular passive soil volume is defined by two slip planes with their origin at the toe of the T-Wall (Figure B-4). Each plane extends from the toe and is defined using the angle given by Equation 2.20, with reference to horizontal. For a compacted, cohesionless engineered backfill consisting of granular soil, this approximate slip plane angle is equal to $30 \mathrm{deg}$. The inverted, triangular volume of passive soil also extends into the soil of the Resisting Wedge, as previously stated. The value for $X_{L-1}^{*}$ will be computed from the $\delta L$ value based upon the user defined material properties of $\phi^{\prime} L$ and the ratio of $\delta_{L} / \phi^{\prime} L$.

$$
X_{L-1}^{*}=E_{L-1}^{*} * \tan \left(\delta_{\text {mob-Passive }}^{\prime}\right)
$$

By Equations B.61 and B.65,

$$
X_{L-1}^{*}=X_{L-1}
$$

Vertical force equilibrium applied to the Figure B-6 soil block - left results in the effective force $N^{\prime} b L$ normal to the base of soil block - left being defined as

$$
N_{b L}^{\prime}=W_{b L}-X_{L-1}+X^{*}{ }_{L 1}-U_{b L}
$$

Moment equilibrium applied to the Figure B-6 soil block - left results in the position of the effective force $N^{\prime} b L$ normal to the base of soil block - left, as measured from point $D_{T o p}$ at the top of the base slab, being defined as

$$
\begin{gathered}
x_{N b L}=\left\{W_{b L} * x_{W b L}+E_{L-1} *\left(h_{E L-1}-h_{t o e}\right)+H_{L-1} *\left(h_{H L-1}-h_{t o e}\right)+H_{L-2} *\left(h_{H L-2}-\right.\right. \\
\left.h_{t o e}\right)+X^{*}{ }_{L-1} *\left(L_{t o e}\right)-E^{*}{ }_{L-1} *\left(h_{E^{*} L-1}-h_{t o e}\right)-H^{*}{ }_{L-1} *\left(h_{H^{*} L-1}-h_{t o e}\right)-H^{*}{ }_{L-2} * \\
\left.\left(h_{H^{*} L-2}-h_{t o e}\right)-U_{b L} *\left(x_{U b L}\right)\right\} / N_{b L}^{\prime}
\end{gathered}
$$

With the symmetry in earth pressure and water pressure distributions, Equation B.65 reduces to

$$
x_{N b L}=\left\{W_{b L} * x_{W b L}++X_{L-1}^{*} *\left(L_{t o e}\right)-U_{b L} *\left(x_{U b L}\right)\right\} / N_{b L}^{\prime}
$$

The weight of soil block - left is given by

$$
W_{b L}=\gamma_{\text {water }} *\left[\left(h_{5}-h_{3}\right) * L_{\text {toe }}\right]+\gamma_{\text {saturated }} *\left[\left(h_{3}-h_{\text {toe }}\right) * L_{\text {toe }}\right](\mathrm{B} .
$$


Its positon $x_{W b L}$ relative to the toe of the base slab is

$$
x_{W b L}=\left\{\begin{array}{c}
\gamma_{\text {water }} *\left[\left(h_{5}-h_{3}\right) * L_{\text {toe }}\right] *\left[\frac{1}{2} * L_{\text {toe }}\right]+ \\
\gamma_{\text {saturated }} *\left[\left(h_{3}-h_{\text {toe }}\right) * L_{\text {toe }}\right] *\left[\frac{1}{2} * L_{\text {toe }}\right]
\end{array}\right\} / W_{b L}
$$

In the case of level, submerged ground, $x_{W b L}$ will be equal to

$$
x_{W b L}=L_{t o e} / 2
$$

With a horizontal phreatic surface within soil block - left, the uplift water pressure acting normal to the base of this soil block will be uniform. With a uniform upward acting seepage gradient $i_{y-R W}$ within the soil block - left, the resultant uplift force $U_{b L}$ is

$$
U_{b L}=\left[\gamma_{\text {water }} *\left(1+i_{y-R W}\right) *\left(h_{3}-h_{\text {toe }}\right)\right] * L_{\text {toe }}
$$

Its positon $x U b L$ relative to the top of the toe slab is

$$
x_{U b L}=L_{t o e} / 2
$$

The horizontal effective earth pressure force $E_{L-1}^{*}$ acts on the stem at a height above the base slab equal to

$$
\left(h_{E^{*} L-1}-h_{\text {toe }}\right)=\frac{\left\{\left[\begin{array}{c}
\left.\frac{1}{2} * \gamma_{1} *\left(h_{3}-h_{\text {toe }}\right)^{2}\right] * \\
{\left[\frac{1}{3} *\left(h_{3}-h_{\text {toe }}\right)\right]}
\end{array}\right\}\right.}{\left\{\left[\frac{1}{2} * \gamma_{1} *\left(h_{3}-h_{\text {toe }}\right)^{2}\right]\right\}}
$$

with $\gamma_{1}$ provided by Equation 2.19 for a constant vertical, upward seepage. This equation simplifies to

$$
\left(h_{E^{*} L-1}-h_{\text {toe }}\right)=\frac{1}{3} *\left(h_{3}-h_{\text {toe }}\right)
$$

The horizontal water pressure force $H^{*}{ }_{L-1}$ acts normal to the stem and at a height above the base slab equal to

$$
\left(h_{H^{*} L-1}-h_{\text {toe }}\right)=\left(h_{5}-h_{\text {toe }}\right) / 3
$$

Figure B-7 shows the free body diagram summarizing the resultant forces acting on the stem and base slab of the Structural Wedge. These forces are 
transferred to the reinforced concrete T-Wall through soil block - right and through soil block - left.

Figure B-7. Free body diagram of resultant forces acting on the stem and base slab of the Structural Wedge.

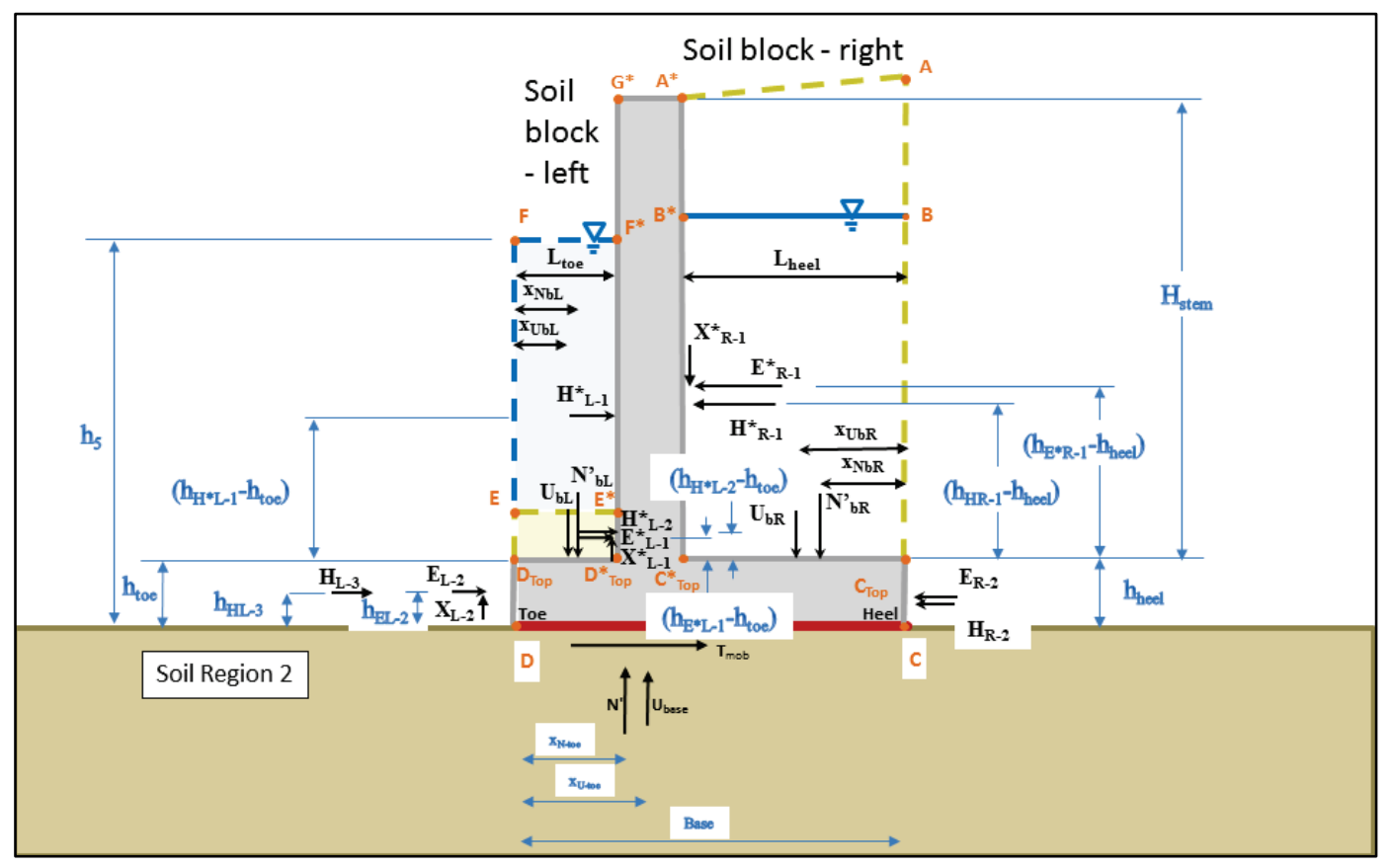

The resultant shear force $V_{\text {stem }}$ acting internal to the base of the reinforced concrete stem is given by

$$
V_{\text {stem }}=-E_{R-1}^{*}-H_{R-1}^{*}+E_{L-1}^{*}+H_{L-1}^{*}+H_{L-2}^{*}
$$

and the moment $M_{\text {stem }}$ acting internal to the base of the reinforced concrete stem is

$$
M_{\text {stem }}=\left\{\begin{array}{c}
E_{R-1}^{*} *\left(\left(h_{E^{*} R-1}-h_{\text {heel }}\right)+H_{R-1}^{*} *\left(h_{H^{*} R-1}-h_{\text {heel }}\right)-\right. \\
E_{L-1}^{*} *\left(h_{E^{*} L-1}-h_{\text {heel }}\right)-H_{L-1}^{*} *\left(h_{H^{*} L-1}-h_{\text {heel }}\right)- \\
H_{L-2}^{*} *\left(h_{H^{*} L-2}-h_{\text {heel }}\right)
\end{array}\right\}
$$




\section{Appendix C: Numerical Methods for the Calculation of $\beta$ and Load and Resistance Factors Given Uncorrelated Load and Resistance PDF Curves}

\section{C.1 Introduction: Assumptions and requirements for calculating load and resistance factors}

This appendix begins by outlining the steps in a numerical procedure for calibrating partial load and resistance safety factors given a target reliability index $(\beta)$ value as well as two alternative procedures of analysis. The first is a numerical procedure described in Section C.2 of this appendix that has been adapted to this R\&D effort from that outlined in Section 8.5 of Nowak and Collins (2013) for bridge design. A numerical procedure to calculate $\beta$ given variable load and resistance with distributions, which may have partial load and resistance safety factors applied is presented to be used as a verification step (Section C.4). To verify the computation of partial safety factors, this procedure is used by calculating $\beta$ from the partial load and resistant factors applied to the load and resistance distributions. This value should then match the target $\beta$ value. This second numerical procedure is also adapted to this $\mathrm{R} \& \mathrm{D}$ effort from a procedure described in Nowak and Collins (2013).

Section C. 3 derives a supplemental procedure to calculate the partial load safety factor given a specified partial resistance safety factor and the procedurally defined partial safety factor ratio. This safety factor ratio may be computed using the numerical procedure outlined in Section C.2.

Section C.5 summarizes a reliability-based numerical procedure for computing a value for Reliability Index $\beta$ following the steps outlined in Nowak (1999). Professor Nowak originally developed this procedure over a number of years with a focus on its application to LRFD based bridge design (Nowak and Lind 1979; Nowak 1999; Nowak and Collins 2000, 2013).

Section C.6 describes a Gaussian function superposition approach of numerically fitting normalized normal distribution functions to a non- 
normal PDF and computing a value for Reliability index $\beta$. The attractiveness of this analysis procedure is that it may be used on any form of PDF yet be able to determine a value for $\beta$ that may be used in a Reliability analysis for determination of load and resistance factors.

These numerical procedures outlined in this appendix require a mathematically defined, continuous probability density function (PDF) distributions for load and resistance. These procedures also require that load and resistance variables not be correlated (i.e., independent).

On page 269 of Nowak and Collins (2013), the LRFD design equation is given as

$$
\gamma_{R} * \mu_{R} \geq \gamma_{L} * \mu_{L}
$$

with $\gamma_{R}$ being the resistance factor, $\mu_{R}$ the mean resistance, $\gamma_{L}$ the load factor and $\mu_{\mathrm{L}}$ the mean load. Note that Nowak and Collins (2000, 2013) are using the mean values for resistance and load and not the nominal values in this governing LRFD equation. This governing equation was subsequently re-written by Nowak and Collins in terms of nominal resistance and load and given on their page 269 as

$$
\left(\gamma_{R} * \lambda_{R}\right) * R_{n} \geq\left(\gamma_{L} * \lambda_{L}\right) * L_{n}
$$

with $\lambda_{R}$ being the resistance bias factor, $R_{n}$ the nominal resistance, $\lambda_{L}$ the load bias factor and $L_{n}$ the nominal load.

As noted, the following sections provide procedures to compute Reliability Indices and partial safety factors. These calculations deal with different types of probability distributions. There are certain conditions that affect the calculation of partial safety factors for loads $(\gamma)$ and partial safety factors for resistances $(\phi)$. For a set of uncorrelated variables described by normal distributions, the procedure is straightforward. Conversion of the normal distributions into a Gaussian Normal space allows for the determination of a design point for the limit state function $g\left(x^{*}\right)$. The closest point from the means to the limit state response surface where $g\left(x^{*}\right)=0$ is the design point. In the Gaussian Normal space, the unit distance corresponds to a change of one standard deviation from the mean origin. Therefore, the distance to the design point gives the $\beta$ distance to the response surface from which $\operatorname{PDF}(\phi)$ and $\operatorname{CDF}(\Phi)$ values can be obtained. 
The vector from the mean origin of each of the $i^{\text {th }}$ variable, $\mu_{i}$, to the design point $x^{*} i$ on the limit state response surface after being normalized to unit length gives the directional cosine components $(\alpha)$ for the uncorrelated variables. Recall $\sigma_{i}$ is the standard deviation for the $i^{\text {th }}$ variable. The design point for $g\left(x^{*}\right)=0$ in variable space is given by the equation

$$
x^{*}{ }_{i}=\mu_{i}+\alpha_{i} \beta \sigma_{i}
$$

Substituting the target $\beta_{\text {target }}$ for the design point $\beta$ projects the response surface to $\beta$ target. Then the factor that gives that value is calculated as

$$
\gamma_{i}=\frac{x_{i}^{*}}{x_{i}^{N}}
$$

Where $\gamma_{i}$ is the factor and $X_{i}$ is the component value given for $\beta_{\text {target, }}$, and $x^{N_{i}}$ is the nominal value for the variable (load or resistance), which can change through the iterative procedure. For resistance, $\gamma_{i}$ becomes $\phi_{i}$ in Equation C.4.

The next section describes the numerical procedure for performing this same set of steps when some or all of the variables possess non-normal distributions.

\section{C.2 Procedure for calculating load and resistance factors given variables with non-normal distributions}

The following steps outline the procedure for calculating load and resistance factors given variables with non-normal distributions.

1. Formulate the limit state function and the design equation. These equations will work with as many random variables as possible, with their distributions and parameters. It is assumed that every random variable will have a COV or standard deviation. At most, only two unknown means will be allowed. Typically, the resistance variable accounts for one unknown mean with the other unknown mean corresponding to one of the load terms. Load ratios set the relationships of the mean values of the loads to the unknown values. For example, in LRFD application to bridge design it is common for the live load to be set equal to one-third the dead load (Nowak and Collins 2013) when applying this numerical procedure. Initializing for the first iteration, the 
limit state equation where $g(\boldsymbol{X})=0$ is evaluated to determine the relationship between the unknown mean values.

2. An initial design point $\boldsymbol{X}$ is obtained by assuming values for $n-1$ of the random variables, with mean values being a good assumption. Solve the limit state equation where $g(\boldsymbol{X})=0$ to determine the remaining variable on the failure boundary.

3. For non-normal axis design point values, determine the equivalent distribution $\left(\mu^{e} X\right.$ and $\left.\sigma^{e} X\right)$ using Equation C.5 and Equation C.6. These values create a distribution at $x^{*}$ that has an equivalent $\mathrm{CDF}$ and PDF at the design point $x^{*}$. If the axis of a design point variable is on a normal distribution, then $\mu^{e} X$ and $\sigma_{X}{ }$ ) correspond to the already existent distribution. This step may not be possible if the normal mean is one of the unknowns.

$$
\begin{gathered}
\mu_{x}^{e}=x^{*}-\sigma_{x}^{e}\left[\Phi^{-1}\left(F_{x}\left(x^{*}\right)\right)\right] \\
\sigma_{x}^{e}=\frac{1}{f_{x}\left(x^{*}\right)} \phi\left(\frac{x^{*}-\mu_{x}^{e}}{\sigma_{x}^{e}}\right)=\frac{1}{f_{x}\left(x^{*}\right)} \phi\left[\Phi^{-1}\left(F_{x}\left(x^{*}\right)\right)\right]
\end{gathered}
$$

4. Calculate the partial derivatives of the limit state function with respect to the reduced variates. The column vector $\{\boldsymbol{G}\}$ is comprised of these derivatives:

$$
\{G\}=\left\{\begin{array}{c}
G_{1} \\
G_{2} \\
G_{3} \\
\cdot \\
\vdots \\
G_{n}
\end{array}\right\} \text {, where } G_{i}=\left.\frac{\partial g}{\partial z_{i}}\right|_{\text {evaluated at the design point }}
$$

5. From the matrix of correlation coefficients $[\rho]$, calculate the directional cosine column vector $\{\alpha\}$ :

$$
\{\alpha\}=\frac{[\rho]\{G\}}{\sqrt{\{G\}^{T}[\rho]\{G\}}}
$$

with the correlation matrix for a two variable problem given as

$$
[\rho]=\left[\begin{array}{ll}
\rho_{11} & \rho_{12} \\
\rho_{21} & \rho_{22}
\end{array}\right]
$$


The diagonals of the correlation matrix are set to unity, and $\rho_{12}$ is typically equal to $\rho_{21}$. For independent variables $X_{1}$ and $X_{2}$, there is no correlation (i.e., $\rho_{12}=\rho_{21}=0$ ), and the diagonal terms are unity.

6. The new design point in reduced variates for $n-1$ variables is computed using the target $\beta, \beta$ target.

$$
z_{i}^{*}=\alpha_{i} \beta_{\text {target }}
$$

7. Return the new design point in the original coordinate space for the $n-1$ variables using the expected distribution for the variables

$$
x_{i}^{*}=\mu_{X_{i}}^{e}+z_{i}^{*} \sigma_{X_{i}}^{e}
$$

At this point, the remaining random value can be solved for by solving the limit state function so that $g\left(x^{*}\right)=0$. The relationship between the two unknown mean values may have changed, so the relationship can be updated by assuming a bias of 1.o, the nominal $x^{N_{i}}$ becomes equal to $\mu x$.

$$
\gamma_{i}=\frac{x_{i}^{*}}{\mu_{x_{i}}}=\frac{\mu_{x_{i}}+z_{i}^{*} \sigma_{x_{i}}}{\mu_{x_{i}}}=1+z_{i}^{*} \operatorname{COV}_{x_{i}}=1+\alpha_{i} \beta_{\text {target }} \operatorname{COV}_{x_{i}}
$$

Therefore,

$$
\mu_{x_{i}}=\frac{x_{i}^{*}}{1+\alpha_{i} \beta_{\text {target }} \operatorname{COV}_{x_{i}}}
$$

8. Repeat steps 3 to 8 until $\{\alpha\}$ converges.

9. Use Equation C.4 with the converged values to calculate each design factor.

\section{Example C.1 - Dead load bending moment for a beam}

A trial design was performed of a singly reinforced beam with an unfactored dead load bending moment, $M_{D L}$, set equal to 200 kip-ft. It follows the procedure of Example 5.9 of Nowak and Collins (2013). This example only deals with a dead load condition. The beam with a width of $12 \mathrm{in}$. and a depth $(d)$ to center of reinforcement of $28 \mathrm{in}$. was analyzed in a 2016 USACE LRFD study (Hokens). A concrete compressive strength, $f_{c}^{\prime}$, of 4,000 psi and a reinforcement yield strength, $f_{y}$, of 60,000 psi were used. The required area of steel, using a resistance factor, $\phi_{A C I}$, of 0.9 from ACI $318-14$, is $2.37 \mathrm{in}^{2}$. 
The load factor for dead load, when not combined with other loads, is 1.4 in ASCE 7-10 and is designated $\gamma_{A S C E}$ in this example. Ellingwood states that the probability density function (PDF or described functionally as $\phi())$ for dead loads applied to buildings is normally distributed with a bias factor, $\lambda_{L}$, of 1.05 and a $C O V_{L}$ of 0.1. The mean dead load, $\mu_{L}$, is computed to be 210 kip-ft by

$$
\mu_{D L}=\lambda_{D L} * M_{D L}
$$

The safety margin relationship for LRFD for this example is

$$
\phi_{A C I} * M_{n}=\gamma_{A S C E} * M_{D L}
$$

Rearranging for $M_{n}$, this equation becomes

$$
M_{n}=\frac{\gamma_{A S C E} * M_{D L}}{\phi_{A C I}}
$$

Introducing the ASCE dead load factor, $\gamma A S C E$, of 1.4, the dead load moment, $M_{D L}$, of 200 kip-ft and the ACI flexural resistance factor, $\phi_{A C I}$, of 0.9, the nominal moment are computed as

$$
M_{n}=\frac{1.4 * 200}{0.9}=311.11 \text { kip-ft }
$$

By the following two relationships for the nominal flexural capacity of a singly reinforced beam equal to $311.11 \mathrm{kip}-\mathrm{ft}$, the required reinforcement steel cross-sectional area is verified (Hokens) to be $2.37 \mathrm{in}^{2}$ for the signally reinforced beam using the relationships

$$
M_{n}=A_{f} * f_{y}\left[d-\frac{a}{2}\right]
$$

with

$$
a=\frac{A_{f^{*}} * f_{y}}{0.85 * f_{c}^{\prime} * b}
$$

where 
$A_{s}=$ area of reinforcing steel

$f_{y}=$ yield strength of reinforcing steel

$d=$ distance from compression face to center of reinforcing steel

$a=$ height of equivalent Whitney stress block in reinforced concrete

$f_{c}=$ concrete compression strength

$b=$ width of beam or design section.

The mean resistance, $\mu_{R}$, is computed to be 348.44 kip-ft by

$$
\mu_{R}=\lambda_{R} * M_{n}
$$

where the bias factor on flexural resistance, $\lambda_{R}$, is defined as 1.12 by Ellingwood for a one-way slab. The $C O V_{R}$ is defined as 0.14. Recall that $M_{n}$ is the nominal resistance computed using equation C.16. Ellingwood also suggests a lognormal distribution for flexural capacity of one-way slabs with a $C O V_{R}$ of 0.14 in his document.

Following the procedural steps for calculating load and resistance factors given variables with non-normal distributions for the first iteration:

1. There are two variables in this example: the resistance variable $(R)$ and the load variable $(L)$. The design equation is defined by $g\left(x^{*}\right)=R-L$, and the limit state occurs when $g\left(x^{*}\right)=0.0$, which occurs when $R=L$. The distribution for the load $L$ is the same as the normal distribution, so the $C O V_{L}=0.1$. It is assumed that both mean load and resistance are unknown. The unknown mean load $\mu_{L}$ is used as the basis for calculations, with all of the other values relating to its value through ratios. This relationship is assumed to be $\mu_{R}=1 \mu_{L}$ for $g\left(x^{*}\right)=0.0$.

2. An initial design point is calculated by assuming $n-1$ variable values. For the two-variable problem, the design point value for load $\left(l^{*}\right)$ is assumed to be the mean value, $1 \mu \mathrm{L}$. Solving the $g\left(x^{*}\right)=0.0$ limit state response surface equation gives the design point for the resistance $\left(r^{*}\right)$ as $1 \mu \mathrm{L}$.

3. $R$ is the only variable with a non-normal distribution, so its equivalent normal distribution values need to be determined using Equation C.5 and Equation C.6. Some additional information is needed from the original log-normal distribution values. From the original log normal distribution (and these values are constant through the iterations),

$$
\sigma_{\ln R}^{2}=\ln \left(1+\frac{\sigma_{R}^{2}}{\mu_{R}^{2}}\right)=\ln \left(1+\frac{48.78^{2}}{348.44^{2}}\right)=0.01941
$$




$$
\begin{gathered}
\sigma_{\ln R}=\sqrt{\sigma_{\ln R}^{2}}=0.139 \\
\mu_{l n R}=\ln \left(\mu_{R}\right)-\frac{\sigma_{\ln R}^{2}}{2}=5.844
\end{gathered}
$$

For log-normal resistance variable, the closed form solution of these equivalent normal parameters are

$$
\begin{gathered}
\sigma_{R}^{e}=r^{*} \sigma_{\ln R}=0.139 \mu_{L} \\
\mu_{R}^{e}=r^{*}\left[1-\ln \left(r^{*}\right)+\mu_{\ln R}\right]=6.844 \mu_{L}
\end{gathered}
$$

Notice that $\mu \ln (R)$ is approximately equal to $\ln \left(\mu_{R}\right)$ when the $C O V_{R}<0.2$ (Nowak and Collins 2013 p 26).

4. Calculating $\{G\}$ from the derivatives,

$$
\begin{gathered}
G_{1}=-\left.\frac{\partial g}{\partial R} \sigma_{R}^{e}\right|_{\text {design point }}=-\sigma_{R}^{e}=-0.139 * \mu_{L} \\
G_{2}=-\left.\frac{\partial g}{\partial L} \sigma_{L}^{e}\right|_{\text {design point }}=\sigma_{L}^{e}=\sigma_{L}=C O V_{L} * \mu_{L}=0.1 * \mu_{L}
\end{gathered}
$$

5. From the matrix equation C.8,

$$
\{\alpha\}=\left\{\begin{array}{l}
\alpha_{R} \\
\alpha_{L}
\end{array}\right\}=\left\{\begin{array}{c}
-0.812 \\
0.583
\end{array}\right\}
$$

Note that since the variables $R$ and $L$ are independent variables, the correlation matrix $[\rho]$ is an identity matrix, with values of 1 along the major term diagonals and off-diagonal terms of $\mathrm{o}$.

6. The new design point in Gaussian Normal coordinates for the load is determined, with $\beta_{\text {target }}=3.0$,

$$
z_{L}^{*}=\alpha_{L} \beta_{\text {target }}=0.583 * 3.0=1.75
$$

7. Returning to the original coordinate system,

$$
l^{*}=\mu_{L}+z_{L}^{*} \sigma_{L}=\mu_{L}\left(1+z_{L}^{*} \operatorname{COV}_{L}\right)=1.175 * \mu_{L}
$$

8. Using $g\left(x^{*}\right)=0.0$, then, 


$$
r^{*}=l^{*}=1.175 * \mu_{L}
$$

An updated value of the estimated mean value of $R$,

$$
\mu_{R}=\frac{r^{*}}{1+\alpha_{R} \beta \operatorname{COV}_{R}}=\frac{1.175 * \mu_{L}}{1+(-0.812)(3)(0.14)}=1.783 * \mu_{L}
$$

9. Table C-1 summarizes how the values change for each iteration of the procedure. The iterative computations for this example were made in an Excel spread sheet. While four iterations were performed, in actuality, a threshold for convergence would be established when implemented within an algorithm.

Table C-1. Iterations of Step 3-8 as the design point and mean resistance change.

\begin{tabular}{|l|l|l|l|l|l|}
\hline & ORIGINAL & ITERATION 1 & ITERATION 2 & ITERATION 3 & ITERATION 4 \\
\hline$r^{*}$ & $1 \mu_{L}$ & $1.174933 \mu_{L}$ & $1.156396 \mu_{L}$ & $1.158209 \mu_{L}$ & $1.15803 \mu_{L}$ \\
\hline$I^{*}$ & $1 \mu_{L}$ & $1.174933 \mu_{L}$ & $1.156396 \mu_{L}$ & $1.158209 \mu_{L}$ & $1.15803 \mu_{L}$ \\
\hline$\mu_{R}$ & $1 \mu_{L}$ & $1.783459 \mu_{L}$ & $1.802396 \mu_{L}$ & $1.80083 \mu_{L}$ & $1.800991 \mu_{L}$ \\
\hline
\end{tabular}

10. Combining Equation C.4 with Equation C.19. the resistance factor is calculated using the resistance bias value for $\lambda_{R}$ value attributed to Ellingwood, as

$$
\gamma_{R}=\phi_{R}=\frac{\lambda_{R} r^{*}}{\lambda_{R} M_{n}}=\frac{\lambda_{R} r^{*}}{\mu_{R}}=\frac{1.12 * 1.15803 * \mu_{L}}{1.800991 * \mu_{L}}=0.72
$$

Recall $\gamma_{R}$ is also designated as $\phi_{R}$ or simply $\phi$ in the main body of this report.

Combining Equation C.4 with Equation C.14, the load factor is calculated using the load bias value for $\lambda_{\mathrm{L}}$ attributed to Ellingwood, as

$$
\gamma_{L}=\frac{\lambda_{L} l^{*}}{\lambda_{L} M_{D L}}=\frac{\lambda_{L} l^{*}}{\mu_{L}}=\frac{1.05 * 1.15803 * \mu_{L}}{1 * \mu_{L}}=1.216
$$

Recall that by applying this numerical procedure outlined in this example guarantees minimal partial resistance and load safety factors that, when multiplied by the nominal resistance and its resistance bias and nominal load and its load bias, will compute a design point on the limit state response surface at distance $\beta$ target. 
In Chapter 4 of the main report, the concept of a $L_{S F}$ was introduced as the scale factor that could be applied to the mean load to create a new mean resistance that, when the $\mathrm{Gz}($ ) function has been applied to the distributions, returns a target PUP value. Introducing these $\gamma_{R}$ and $\gamma_{L}$ values of 0.72 and 1.216, respectively, into Equation 4.6, the $L_{S F}$ is

$$
L_{S F}=\frac{\gamma_{L}}{\gamma_{R}}=\frac{1.216}{0.72}=1.69
$$

Note that because $L_{S F}$ was determined in the Nowak and Collins (2013) procedure of analysis using an LRFD formulation written in terms of mean values for the load and resistance, this constant does not work with nominal loads and resistances without some modification. This modification for use with nominal values of loads and resistances is discussed in Subsection C.4.2.

\section{C.3 Supplemental procedure: calculation of the partial load safety factor given a specified partial resistance safety factor and the procedurally defined partial safety factor ratio}

The limit state $g(\boldsymbol{X})$ function is defined for $i$ resistances and $j$ loads as

$$
\sum_{i}\left(\gamma_{R_{i}} * \mu_{R_{i}}\right) \geq \sum_{j}\left(\gamma_{L_{j}} * \mu_{L_{j}}\right)
$$

For the single load and resistance problem and because $g(\boldsymbol{X})=0.0$ at the limit state response surface where $R-L=0.0$,

$$
\begin{gathered}
\gamma_{R} * \mu_{R}=\gamma_{L} * \mu_{L} \\
\mu_{R}=\frac{\gamma_{L}}{\gamma_{R}} * \mu_{L}
\end{gathered}
$$

Note that in this Nowak and Collins (2013) based procedure of analysis, these equations make use of mean values for loads and resistances rather than nominal values for loads and resistances. Introducing Equation C.19 and C.14 into Equation C.22, the relationship between nominal values of load and resistance is

$$
M_{N} * \lambda_{R}=\frac{\gamma_{L}}{\gamma_{R}} * L_{N} * \lambda_{L}
$$

Solving for $M_{N}$, 


$$
M_{N}=\frac{\gamma_{L} * \lambda_{L}}{\gamma_{R} * \lambda_{R}} * L_{N}
$$

This implies that there is a $\gamma_{\text {Lnominal }}$ and a $\gamma_{\text {Rnominal }}$ that may be computed as

$$
\begin{gathered}
\gamma_{\text {Lnominal }}=\gamma_{L} * \lambda_{L} \\
\gamma_{\text {Rnominal }}=\gamma_{R} * \lambda_{R}
\end{gathered}
$$

The nominal scale factor $L_{S F N o m i n a l}$ can then be defined as

$$
L_{\text {SFNominal }}=\frac{\gamma_{\text {Lnominal }}}{\gamma_{\text {Rnominal }}}
$$

The initial value for nominal resistance $M_{N}$ was calculated from the nominal load $L_{N}$ based on an unbiased ASCE load factor $\gamma_{A S C E}$ and an ACI resistance factor $\gamma_{A C I}$ (which is the same as $\phi_{A C I}$ ) specified in the Hokens writeup:

$$
M_{N}=\frac{\gamma_{A S C E}}{\phi_{A C I}} * L_{N}
$$

This implies that the ratio of the factors is a constant for the single load and resistance problem because the ratio of the means is a constant at the limit state response surface. Recall from example C.1 the values for $\gamma_{R}$ and $\gamma_{L}$ are 0.72 and 1.216, respectively. Also, recall the values of the bias factors $\lambda_{L}$ and $\lambda_{R}$ are 1.05 and 1.12, respectively. In keeping with the specified flexural resistance factor 0.9 of ACI 318-14, which is applied to the nominal resistance, and maintaining the same relationship between the load and resistance factor to satisfy the $g(\boldsymbol{X})=0$ limit state response surface equation (assuming the mean load and resistance values do not change), then the load factor according to an ACI 318-14 $\phi_{A C I}$ value of 0.9 should account for bias factors using nominal values and be

$$
\begin{gathered}
\frac{\gamma_{A S C E}}{\phi_{A C I}}=\frac{\gamma_{L} * \lambda_{L}}{\gamma_{R} * \lambda_{R}} \\
\gamma_{A S C E}=\phi_{A C I} \frac{\gamma_{L} * \lambda_{L}}{\gamma_{R} * \lambda_{R}} \\
\gamma_{A S C E}=0.9\left(\frac{1.216 * 1.05}{0.72 * 1.12}\right)=1.425
\end{gathered}
$$


This $\gamma_{A C I}$ load factor value shows a less than $2 \%$ difference from the 1.4 value which was calculated and reported in the Hokens write-up of LRFD procedures for this singly reinforced beam example problem.

An $L_{S F}$ value of 1.69 was calculated in the previous subsection. This value corresponds to the case of mean load and resistance values. A LSFNominal value can be obtained from the relationship in Equation C.27:

$$
L_{S F N o m i n a l}=L_{S F} * \frac{\lambda_{L}}{\lambda_{R}}=\frac{\gamma_{L} * \lambda_{L}}{\gamma_{R} * \lambda_{R}}=\frac{1.216 * 1.05}{0.72 * 1.12}=\frac{1.2768}{0.8064}=1.58
$$

Using the $\gamma_{A S C E}$ to $\phi_{A C I}$ relationship gives an engineering load scale factor LSFEngineering value of

$$
L_{S F E n g i n e e r i n g}=\frac{\gamma_{A S C E}}{\phi_{A C I}}=\frac{1.4}{0.9}=1.56
$$

There is just over a $1 \%$ difference between the LSFNominal and LSFEngineering values. They are judged to be consistent.

In summary, the $L_{S F}$ value is based on mean values for resistance and load. To compare an $L_{S F}$ value to a scale factor based on nominal resistance and load (i.e., a $L_{S F E n g i n e e r i n g}$ value), a conversion from $L_{S F}$ to $L_{S F N o m i n a l}$ is required. After conversion, the values for LSFNominal and LSFEngineering may be compared directly.

This supplemental scaling procedure only works for the single load and resistance problem as outlined in this subsection. Keeping the same ratio of load factor to resistance factor guarantees that the factored load will be equivalent to the factored resistance for a design point on the limit state response surface. Recall that the factors were calculated so that the factored resistance and factored load satisfy the $g\left(x^{*}\right)=0$ equation for a design point at $\beta$ target distance from the mean values for load and resistance.

\section{C.4 Verification: procedure for calculating design point $\beta$ at the limit state for given variables with non-normal distributions}

\section{C.4.1 Numerical verification}

The verification procedure for determining the design point $\beta$ for $g\left(x^{*}\right)=0$ given non-normal distributions for the variable space is similar to the 
process for determining load and resistance factors as outlined in Subsection C.2. This follows because the latter procedure is derived from the former (Nowak and Collins 2013), as described in their Subsection 5.4.1. This means that the procedure to calculate the $\beta$ for the design point can act as verification of the load and resistance factors by using factored loads and resistances and checking to make sure the calculated $\beta$ is indeed equal to the target value.

The $g(\boldsymbol{X})$ function provides limit state satisfactory values when the factored load and factored resistance satisfy the following inequality:

$$
\sum_{i}\left(\gamma_{R_{i}} * \frac{\mu_{R_{i}}}{\lambda_{R_{i}}}\right) \geq \sum_{j}\left(\gamma_{L_{j}} * \frac{\mu_{L_{j}}}{\lambda_{L_{j}}}\right)
$$

Recall $\gamma_{R}$ is also designated as $\phi_{R}$ or simply $\phi$ in the main body of this report. Observe that this relationship is expressed in terms of mean values. The limit state response surface occurs when both sides are equivalent.

The formulation steps for the two independent variables problem are the following:

1. Formulate limit state function $g\left(x^{*}\right)$ and probability distributions. These values should match the values used in the load and resistance factor calculations.

2. Guess an initial design point. A good initial guess would be an average of the mean values for load and for resistance.

3. Determine the equivalent normal distribution parameters for the nonnormal distributions at the design point. For instance, for a log-normal distribution of variate $X_{i}$ :

$$
\begin{gathered}
\sigma_{\ln X_{i}}^{2}=\ln \left(1+\frac{\sigma_{X_{i}}^{2}}{\mu_{X_{i}}^{2}}\right) \\
\mu_{\ln X_{i}}=\ln \left(\mu_{X_{i}}\right)-0.5 \sigma_{\ln X_{i}}^{2}
\end{gathered}
$$

Nowak and Collins (2013) derived the following equivalent normal distribution values from Equations C.5 and C.6 for the log-normal distribution, with design point $x^{*}$ :

$$
\sigma_{X_{i}}^{e}=x_{i}^{*} \sigma_{\ln X_{i}}
$$




$$
\mu_{X_{i}}^{e}=x_{i}{ }^{*}\left[1-\ln \left(x_{i}{ }^{*}\right)+\mu_{\ln X_{i}}\right]
$$

Nowak and Collins (2013) also provide other equations for other nonnormal distributions.

4. Transform the variates to the reduced Gaussian normalized space.

$$
z_{i}^{*}=\frac{x_{i}^{*}-\mu_{X_{i}}^{e}}{\sigma_{X_{i}}^{e}}
$$

5. Determine the partial derivatives of the slope of the $g\left(x^{*}\right)$ function for each variate and store in a $\{\boldsymbol{G}\}$ vector. For a limit state that only has a load and resistance and $g\left(x^{*}\right)=X_{1}-X_{2}$ (where $X_{1}$ corresponds to the resistance and $X_{2}$ corresponds to the load). From Equation C.7:

$$
\begin{aligned}
& G_{1}=-\left.\frac{\partial g}{\partial X_{1}}\right|_{X_{1}{ }^{*}}=-1 \sigma_{X_{1}}^{e} \\
& G_{2}=-\left.\frac{\partial g}{\partial X_{2}}\right|_{x_{2}{ }^{*}}=+1 \sigma_{X_{2}}^{e}
\end{aligned}
$$

Observe the negative sign in front of the partial in both of these equations.

6. From the reduced variates and the vector $\{G\}$, estimate $\beta$.

$$
\beta=\frac{\{G\}^{T}\left\{z^{*}\right\}}{\sqrt{\{G\}^{T}\{G\}}}
$$

For this uncorrelated two variable problem, the Equation C.9

correlation matrix $[\rho]$ is an identity matrix, which by definition does not change the vector it is multiplied against. In this case, the transposed matrix Equation C.8 times the vector $\left\{z^{*}\right\}$ simplifies to the matrix equation form given here to determine the scalar value of $\beta$.

7. The directional cosine values $\alpha$ are then calculated using Equation C.8. It is generally assumed that the variables are uncorrelated.

8. The Gaussian Normalized design point is calculated for the variables using $\alpha$ and $\beta$ values for $n-1$ variables, which are on the response surface based on the equivalent normal distribution.

$$
z_{i}^{*}=\alpha_{i} \beta
$$


9. Convert the Gaussian Normalized design point back to the variable space for the $n-1$ variables.

10. For the unknown variable, determine the value such that the position in non-normalized variable space satisfies the limit state function where $g\left(x^{*}\right)=0$.

11. Iterate until the value of $\beta$ and the design point $x^{*}$ converge. Because the variable spaces have non-normal distributions, this convergence step needs to occur even if the limit state function is linear.

Example C.2 - verifying example C.1 results

This section is a continuation of the Example C.1 problem, including its results. It follows the procedure of Example 8.4 of Nowak and Collins (2013).

1. This step has been performed in Example C.1.

2. The mean load $\mu_{L}$ is specified as 210 kip-ft based on the nominal value, $\mathrm{N}_{\mathrm{L}}$, equal to $200 \mathrm{kip}$-ft and a load bias factor $\lambda_{L}$ equal to 1.05. The load $C O V_{L}=0.1$. The log-normal resistance distribution has a $C O V_{R}=0.14$ (Ellingwood) and has a resistance bias factor $\lambda_{R}$ equal to 1.12. The load and resistance factors $\gamma_{L}$ and $\gamma R$ are drawn from the previous example with values of 1.216 and 0.72 , respectively. The mean resistance $\mu_{R}$ is calculated (using the equivalency expressed in the relationship of Equation C.21) as

$$
\begin{aligned}
& \mu_{L}=N_{L} * \lambda_{L}=210.00 \\
& \mu_{R}=\left(\frac{\mu_{L} * \gamma_{L}}{\gamma_{R}}\right)=354.67 \\
& \sigma_{L}=\mu_{L} * \operatorname{COV}_{L}=21.00 \\
& \sigma_{R}=\mu_{R} * \operatorname{COV}_{R}=49.64
\end{aligned}
$$

Therefore, the initial guess of the design point gives a value of

$$
r^{*}=(210+354.57) / 2=282.29
$$

Given $g\left(x^{*}\right)=R-L=0$ :

$l^{*}=282.29$ 
3. The equivalent normal parameters can be calculated in the same manner as Example C.1, resulting in

$$
\begin{aligned}
& \sigma_{\ln R}=\sqrt{\ln \left(1+\frac{\sigma_{R}^{2}}{\mu_{R}^{2}}\right)}=\sqrt{\ln \left(1+\frac{49.64^{2}}{354.57^{2}}\right)}=0.139 \\
& \mu_{\ln R}=\ln \left(\mu_{R}\right)-\frac{\sigma_{\ln R}^{2}}{2}=\ln (354.57)-\frac{0.139^{2}}{2}=5.861
\end{aligned}
$$

and an equivalent normal distribution for resistance at the design point given by

$$
\begin{aligned}
& \sigma_{R}^{e}=r^{*} \sigma_{\ln R}=39.33 \\
& \mu_{R}^{e}=r^{*}\left[1-\ln \left(r^{*}\right)+\mu_{\ln (R)}\right]=343.9
\end{aligned}
$$

Notice that $\mu_{\ln (R)}$ is approximately equal to $\ln \left(\mu_{R}\right)$ when the $\mathrm{COV}_{\mathrm{R}}<0.2$ (Nowak and Collins 2013, p 26).

The equivalent normal distribution for the load at the design point is the existing distribution calculated in Step 2:

$$
\begin{aligned}
& \sigma_{L}^{e}=21 \\
& \mu_{L}^{e}=210
\end{aligned}
$$

4. The reduced variate design point is calculated with Equation C.37:

$$
\begin{aligned}
& z_{L}^{*}=\frac{l^{*}-\mu_{L}^{e}}{\sigma_{L}^{e}}=\left(\frac{294.104-210}{21}\right)=3.442 \\
& z_{R}^{*}=\frac{r^{*}-\mu_{R}^{e}}{\sigma_{R}^{e}}=\left(\frac{294.104-365.22}{40.975}\right)=-1.567
\end{aligned}
$$

5. Determining the $\{G\}$ vector,

$$
\begin{aligned}
& G_{1}=-\left.\frac{\partial g}{\partial R}\right|_{r^{*}}=-1 \sigma_{R}^{e}=-39.328 \\
& G_{2}=-\left.\frac{\partial g}{\partial L}\right|_{l^{*}}=+1 \sigma_{L}^{e}=21
\end{aligned}
$$

6. Estimate $\beta$ using Equation C.40, $\beta=3.003$.

7. The $\{\alpha\}$ vector is determined using Equation C.8 


$$
\alpha=\left\{\begin{array}{l}
\alpha_{R} \\
\alpha_{L}
\end{array}\right\}=\left\{\begin{array}{c}
-0.882 \\
0.471
\end{array}\right\}
$$

with the correlation coefficient matrix $[\rho]$ defined as an identity matrix for the pair of independent variables $R$ and $L$.

8. The new reduced design point for the resistance term is calculated as

$$
z_{R}^{*}=\alpha_{R} \beta=-0.882 * 3.003=-2.65
$$

9. Converting back from the Gaussian Normal space,

$$
r^{*}=\mu_{R}^{e}+z_{R}^{*} * \sigma_{R}^{e}=343.9-2.65 * 39.33=239.708
$$

10. From the limit state response surface equation $g(\boldsymbol{X})=0.0$, $l^{*}=r^{*}=239.708$.

11. Table C-2 shows the results of iterating Steps 3 through 10 four times. The calculations were all made in an Excel spread sheet. This gives a clear idea of the convergence pattern that is approaching a $\beta$ of 2.98, or a difference of less than $1 \%$ from the $\beta$ target values of 3 . The loss of precision may be attributed to the small number of iterations (four) that were performed in the Taylor series approximation of Example C.1 as well as the fact that higher-order terms were neglected in the Taylor series expansion formulation. 
Table C-2. Iterations of Step 3-10 as the design point and $\beta$ values change.

\begin{tabular}{|l|l|l|l|l|l|}
\hline & Original & Iteration 1 & Iteration 2 & Iteration 3 & Iteration 4 \\
\hline$r^{*}$ & 282.2838 & 239.7079 & 244.3495 & 243.8894 & 243.9310736 \\
\hline$I^{*}$ & 282.2838 & 239.7079 & 244.3495 & 243.8894 & 243.9310736 \\
\hline$\beta$ & 3.003365 & 3.07278 & 3.073797 & 3.073807 & 3.073807221 \\
\hline
\end{tabular}

Notice that in Step 2, the factored mean resistance is calculated based on the original mean load per the relationship of Equation C.21. This proves that the calculation of the proportional $\gamma_{L}$ values from the ACI $\gamma_{R}$ values of Step 10 of Example 1 will provide the same values of Table C-2.

Table C-3 shows the calculation of $\beta$ using the verification procedure for four iterations using the nominal load and resistance factors $\left(\gamma_{L}=1.4\right.$ and $\gamma_{R}=0.9$, respectively) as calculated in the Hokens write-up of LRFD procedures.

Table C-3. Iterations of Step 3-10 as the design point and $\beta$ values change for the Hokens write-up of LRFD procedures.

\begin{tabular}{|l|l|l|l|l|l|}
\hline & Original & Iteration 1 & Iteration 2 & Iteration 3 & Iteration 4 \\
\hline$r^{*}$ & 279.2222 & 238.9629 & 243.2369 & 242.8256 & 242.8657985 \\
\hline$I^{*}$ & 279.2222 & 238.9629 & 243.2369 & 242.8256 & 242.8657985 \\
\hline$\beta$ & 2.903375 & 2.966626 & 2.967497 & 2.967504 & 2.967504536 \\
\hline
\end{tabular}

The results reveal that the actual $\beta$ given these factors converges to 2.97, which is approximately $1.1 \%$ less than the desired $\beta$ target value of 3 computed in a Hokens study. The 2016 study computed PUP values using Monte Carlo simulations from the Log-Normal resistance distribution and the Normal load distribution. The PUP value is a cumulative distribution effect of probabilities of $(R-L)$ up to o.o, so one would think that the $\beta$ value could be determined by using the inverse CDF function ( $\left.\Phi^{-1}(P U P)\right)$. This was the procedure used in the 2016 study. Unfortunately, the distribution resulting from subtracting load from resistance $(R-L)$ is not a normal distribution because the resistance distribution is non-normal. To map the non-normal $(R-L)$ distribution to a normal distribution $\beta$ value requires a transform into Gaussian Normal space. This transform is fixed by the constraints that the inverse $\operatorname{CDF}\left(\Phi^{-1}\right)$ and the inverse $\operatorname{PDF}\left(\phi^{-1}\right)$ of the distance from the mean to the design point must have the same $\beta$ value. Recall that the design point is defined by $(R-L)$ equal to zero. This transform moves the mean of the $(R-L)$ non-normal distribution to a 
Gaussian Normal distribution, resulting in a different distance to the design point and therefore a different $\beta$ value. This observation demonstrates the need to follow the numerical procedure outlined in Section C.1 to determine the partial resistance and load safety factor values that are consistent with the $\beta_{\text {target }}$ value and allow for the scaling of the load factor based on any other user-prescribed resistance factor value using the supplemental procedure of Section C.3.

\section{C.4.2 Simulation verification}

Simulation methods can also be used to verify that the load and resistance factors computed in Example C.1 generate the target PUP and $\beta$ values, given a sufficient number of samples to guarantee precision. The mean load has the same value for both the unpublished USACE (2016) procedure and the load and resistance factor procedure. This value is computed as follows

$$
\mu_{L}=L_{N} * \lambda_{L}=200 * 1.05=210
$$

The mean resistance is computed differently depending on the procedure used. The unpublished USACE (2016) procedure calculates the mean resistance value using nominal values:

$$
\mu_{R}=L_{N} * \frac{\gamma_{A S C E}}{\phi_{A C U}} * \lambda_{R}=200 * \frac{1.4}{0.9} * 1.12=348.44
$$

Using the computed load and resistance factors from Example C.1, the computation of mean resistance becomes

$$
\mu_{R}=\mu_{L} * \frac{\gamma_{L}}{\gamma_{R}}=210 * \frac{1.216}{0.72}=354.67
$$

Recall that load uses a normal distribution and that resistance uses a lognormal distribution. Table $\mathrm{C}-4$ shows the distributions and the resultant PUP values for the two procedures simulated with 1,000,000 samples. Both results are very close to the target $b$ value of 3.0, indicating that the results are within the sampling error of the simulations. For these simulations, the engineering values gave a closer approximation to the target $\beta$ value, but both values are within the significant error. 
Table C-4. Comparison of LRFD statistical variable parameters and computed results from the unpublished USACE (2016) letter report and the computations made in this appendix.

\begin{tabular}{|l|l|l|}
\hline & USACE (2016) & LRFD Factors \\
\hline$\mu_{L}$ & 210 & 210 \\
\hline COV $_{L}$ & 0.1 & 0.1 \\
\hline$\sigma_{L}$ & 21 & 21 \\
\hline$\mu_{R}$ & 348.44 & 354.67 \\
\hline $\operatorname{COV}_{R}$ & 0.14 & 0.14 \\
\hline$\sigma_{R}$ & 48.78 & 49.65 \\
\hline$\mu_{L N R}$ & 5.844 & 5.861 \\
\hline$\sigma_{L N R}$ & 0.139 & 0.139 \\
\hline$P U P$ & 0.001418 & 0.001016 \\
\hline$\beta=\Phi^{-1}$ (PUP) & 2.983 & 3.085 \\
\hline
\end{tabular}

\section{C.5 Nowak (1999) Reliability method-based numerical procedure for computing a value for Reliability Index $\beta$}

In 1999 Nowak, introduced a reliability-based numerical procedure for computing a value for Reliability Index $\beta$. The procedure that he outlined in his paper assumed that the total load, $L$, is a normal random variable and that the resistance, $R$, is a lognormal random variable. These assumptions are typical in bridge design. This procedure can be adjusted to work with other distributions if the equations are re-derived. Nowak's procedure is described in the following eight steps:

1. The Nowak (1999) Reliability-based procedure relies on specified inputs:

Resistance parameters: $\quad R_{n}, \lambda_{R}, C O V_{R}$

Load parameters: $\quad \mu Q, \sigma Q$

where $R_{n}$ is the nominal resistance, $\lambda_{R}$ is the resistance bias factor, $C O V_{R}$ is the coefficient of variation for resistance, $\mu_{Q}$ is the mean load, and $\sigma_{Q}$ is the standard deviation of the load.

2. The mean resistance is calculated as

$$
\mu_{R}=\lambda_{R} R_{n}
$$


3. Assume the initial design point for unknown $k$ as

$$
R^{*}=\mu_{R}\left(1-k * C O V_{R}\right)
$$

For the initial value, $k$ is set equal to 2 by Nowak (1999) in his LRFD application to bridge design.

4. Compute the value for the CDF $\Phi$ and PDF $\phi$ of the design point on the lognormal distribution $R$ at the design point $R^{*}$, with the superscripted asterisk designating the design point. This calculation can be sped up by precalculating the argument for functions $\Phi$ and $\phi$,

$$
\alpha^{*}=\left(\ln R^{*}-\ln \mu_{R}\right) / C O V_{R}
$$

so that the CDF and PDF are, respectively,

$$
\begin{gathered}
F_{R}\left(R^{*}\right)=\Phi\left(\alpha^{*}\right) \\
f_{R}\left(R^{*}\right)=\phi\left(\alpha^{*}\right) /\left(C O V_{R} R^{*}\right)
\end{gathered}
$$

5. Using Rackwitz-Fiessler $(1976,1978)$ equations, find the normal distribution for $R^{\prime}$ that approximates the values at $R^{*}$.

$$
\begin{gathered}
\sigma_{R^{\prime}}=\frac{\phi\left\{\Phi^{-1}\left[\Phi\left(\alpha^{*}\right)\right]\right\}}{\left[\frac{\phi\left(\alpha^{*}\right)}{\operatorname{Cov} R^{*}}\right]}=\operatorname{COV}_{R} R^{*} \\
\mu_{R^{\prime}}=R^{*}-\sigma_{R^{\prime}} \Phi^{-1}\left[\Phi\left(\alpha^{*}\right)\right]=R^{*}-\alpha^{*} \sigma_{R^{\prime}}
\end{gathered}
$$

Because the load is defined as a normal curve, the values that describe it are the mean, $\mu_{Q}$, and standard deviation, $\sigma_{Q}$.

6. The Reliability Index, $\beta$, is calculated as

$$
\beta=\left(R^{*}-\alpha^{*} \operatorname{COV}_{R} R^{*}-\mu_{Q}\right) / \sqrt{\left(\operatorname{COV}_{R} R^{*}\right)^{2}+\sigma_{Q}^{2}}
$$

7. A new design point is calculated for the resistance term

$$
R^{*}=\mu_{R^{\prime}}-\beta\left(\operatorname{COV}_{R} R^{*}\right)^{2} / \sqrt{\left(\operatorname{COV}_{R} R^{*}\right)^{2}+\sigma_{Q}^{2}}
$$


8. If the new design point varies significantly from the last assumed value, go to Step 4 and repeat. Typically, the value can be obtained in one cycle.

This numerical procedure will find the value for $k$ that gives a maximum $\beta$ value for the distribution created by subtracting the load distribution from the resistance distribution. The steps above were performed for resistance parameter values of $R_{n}=311.11 \mathrm{kip}-\mathrm{ft}, \lambda_{R}=1.12, C O V_{R}=0.14$ and load parameters of $\mu_{Q}=210 \mathrm{kip}-\mathrm{ft}, \sigma_{Q}=21 \mathrm{kip}-\mathrm{ft}$ for the reinforced concrete beam problem discussed in Section C.2. The nominal load is $200 \mathrm{kip}-\mathrm{ft}$ with $\lambda_{\text {Load }}=1.05$ and the $C O V_{L}=0.1$. The corresponding mean value for resisting moment equals 348.44 kip-ft by Equation C.19. The corresponding mean value for dead load moment equals $210 \mathrm{kip}-\mathrm{ft}$ by Equation C.14. Recall that the nominal resistance is based on ASCE 7-10 load and ACI 318-14 resistance factors. This procedure generated a maximum $\beta$ for safety margin $(R-L)$ of 3.02 with a $k$ value of 2.16 for the Section C.2 beam possessing a $\mu_{R}$ of 348.44 kip-ft.

The resistance parameter value of $R_{n}$ for the reinforced concrete beam was then changed to $=37.68 \mathrm{kip}-\mathrm{ft}$ (for a lognormal distribution with a bias factor of 1.12 and a $C O V_{R}$ of 0.14). The corresponding mean value for resisting moment equals $378.31 \mathrm{kip}-\mathrm{ft}$ by Equation C.19. The nominal value of the dead load moment remains 200 kip-ft (normal distribution) with a dead load bias factor of 1.05 and $C O V_{D L}$ of 0.1 . The corresponding mean value for dead load moment remains unchanged and equal to $210 \mathrm{kip}-\mathrm{ft}$ by Equation C.14. Notice that this nominal resistance value is based on the computed load and resistance factors from Section C.2 as described in Section C.3. This procedure generated a maximum $\beta$ for $R-L$ of 3.52 with a $k$ value of 2.46 .

Nowak and Lind (1979) introduced the concept that the load factor is related to the bias of the design value to the mean value of the load component $\left(\lambda_{i}\right)$, a target $\beta$ with its directional cosine terms ( $\beta$ target $\alpha$, now denoted by Nowak and Lind a designated constant $k$ ), and the coefficient of variation of the load component $\left(\mathrm{COV}_{i}\right)$. For normally distributed loads applied to a design attributing to the total effect, the equation for the load factor $\left(\gamma_{i}\right)$ is therefore

$$
\gamma_{i}=\lambda_{i}\left(1+k * \operatorname{COV}_{i}\right)
$$


For normally distributed resisting loads applied to a design reducing the total effect, the equation for the resistance factor $\left(\phi_{i}\right)$ is therefore

$$
\phi_{i}=\lambda_{i}\left(1-k * \operatorname{COV}_{i}\right)
$$

In Nowak (1999) and Nowak and Lind (1979), load and resistance factors related to $k$ values varying from 1.8-2.1 were used for bridge design, and the load and resistance factors were rounded to the nearest 0.05. Nowak (1999) fixed the value of $k$ to 2.0 for multiple load cases applied to LRFD bridge design with three dead loads, one live load, and one impact load in his Appendix F. He made conservative simplifying assumptions to aid the designer using estimated biases and COVs, altering the resulting load factors. Bathurst et al. (2008) noted that a value of $k=2$ was used in the development of the Canadian highway bridge design code and the AASHTO LRFD bridge design specifications. This value of $k$ was also used by the team of Bathurst, Allen and Nowak in their research into load and resistance factors for reinforced earth applications. The load factors were determined for all of the loads and a conservative value was chosen between the extremes.

Using Equations C.51 and C.52 with $k=2.0$ with the input bias factor values and COVs results in

$$
\begin{aligned}
& \gamma_{L}=\lambda_{L}\left(1+k * \operatorname{COV}_{L}\right)=1.05(1+2.0 * 0.1)=1.26 \\
& \phi_{R}=\lambda_{R}\left(1-k * \operatorname{COV}_{R}\right)=1.12(1-2.0 * 0.14)=0.806
\end{aligned}
$$

The Nowak (1999) and Nowak and Collins (2013) equation that solves for $\beta$ in this procedure given a lognormal resistance distribution and the normal load distribution is therefore

$$
\beta=\frac{R_{n} \lambda_{R}\left(1-k V_{R}\right)\left[1-\ln \left(1-k V_{R}\right)\right]-\mu_{Q}}{\sqrt{\left[R_{n} V_{R} \lambda_{R}\left(1-k V_{R}\right)\right]^{2}+\sigma_{Q}^{2}}}
$$

This equation was also applied to LRFD based tunnel design by the Nowak PhD student Ghasemi in his 2015 Auburn University PhD dissertation (Ghasemi 2015).

Figure $\mathrm{C}-1$ shows the results from using Equation C.51 with resistance characterized by $R_{n}=311.11$ kip-ft with $\mu_{R}$ of 348.44 kip-ft and a nominal load of $200 \mathrm{kip}-\mathrm{ft}$ with $\mu_{Q}=210 \mathrm{kip}-\mathrm{ft}$ and varying $k$ to determine values for 
$\beta$. Notice that these plots verify the maximum $\beta$ s calculated from the procedural method. Table $\mathrm{C}-5$ summarizes the values for $\beta$ in $k$-value range suggested in the 1979 Nowak and Lind paper. In Nowak's later work on LRFD as applied to bridge design (e.g., Nowak (1999), Nowak and Collins (2000, 2013), a $k$ value of 2.0 is used. This same $k$ value of 2.0 was used in his LRFD application to reinforce earth design (Bathurst et al. 2008).

Therefore, attention was focused on the variation in resulting Equation C.51 $\beta$ values for a range in $k$ from 1.8 to 2.1 (Table C-5). For LRFD-based tunnel design, Ghasemi (2015) states that $k$ may be taken approximately 1.8 to 2.0 for strength limit states (Nowak 1999). Observe the minor variation in $\beta$ value within the $k$ equal to 1.8 to 2.1 range.

Figure $\mathrm{C}-1$. Reliability Index $\beta$ as a function of the constant $k$ for the problem of a reinforced concrete beam with its capacity defined by a nominal resisting moment of 311.11 kip-ft with a mean resisting moment of $348.44 \mathrm{kip}-\mathrm{ft}$.

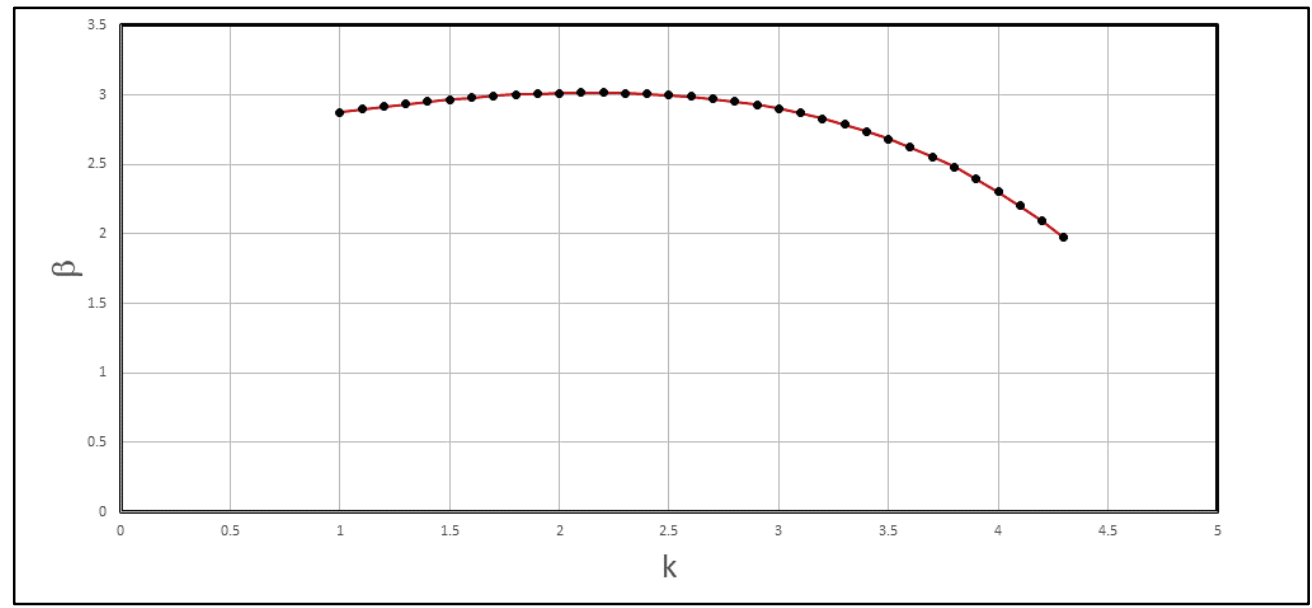




\section{Table C-5. Reliability Index $\beta$ as a function of the constant $k$ for the problem of a reinforced concrete beam with its capacity defined by a nominal resisting moment of 311.11 kip-ft with a mean resisting moment of 348.44 kip-ft.}

\begin{tabular}{|c|c|}
\hline $\mathrm{K}$ & $\boldsymbol{\beta}$ \\
\hline 1.8 & 3.000 \\
\hline 2 & 3.013 \\
\hline 2.1 & 3.016 \\
\hline
\end{tabular}

Recall that the Nowak (1999) numerical procedure discussed earlier in this section generated a maximum $\beta$ for $R-L$ of 3.02 with a $k$ value of 2.16 . Therefore, for an assigned $k$ value of 2 , a $\beta$ value of 3.013 is close to this maximum $\beta$ value from the numerical procedure.

Figure C-2 shows the results from using Equation C.51 with resistance characterized by $R_{n}=337.68 \mathrm{kip}-\mathrm{ft}$ with $\mu R$ of $378.31 \mathrm{kip}-\mathrm{ft}$ and a nominal load of $200 \mathrm{kip}-\mathrm{ft}$ with $\mu Q=210 \mathrm{kip}-\mathrm{ft}$ and varying $k$ to determine values for $\beta$. Notice that these plots verify the maximum $\beta$ s calculated from the procedural method: Table C- 6 summarizes the values for $\beta$ in $k$-value range suggested in the 1979 Nowak and Lind paper. Observe the minor variation in $\beta$ value within the $k$ equal to 1.8 to 2.1 range.

Figure C-2. Reliability Index $\beta$ as a function of the constant $k$ for the problem of a reinforced concrete beam with its capacity defined by a nominal resisting moment of 337.68 kip-ft with a mean resisting moment of 378.31 kip-ft.

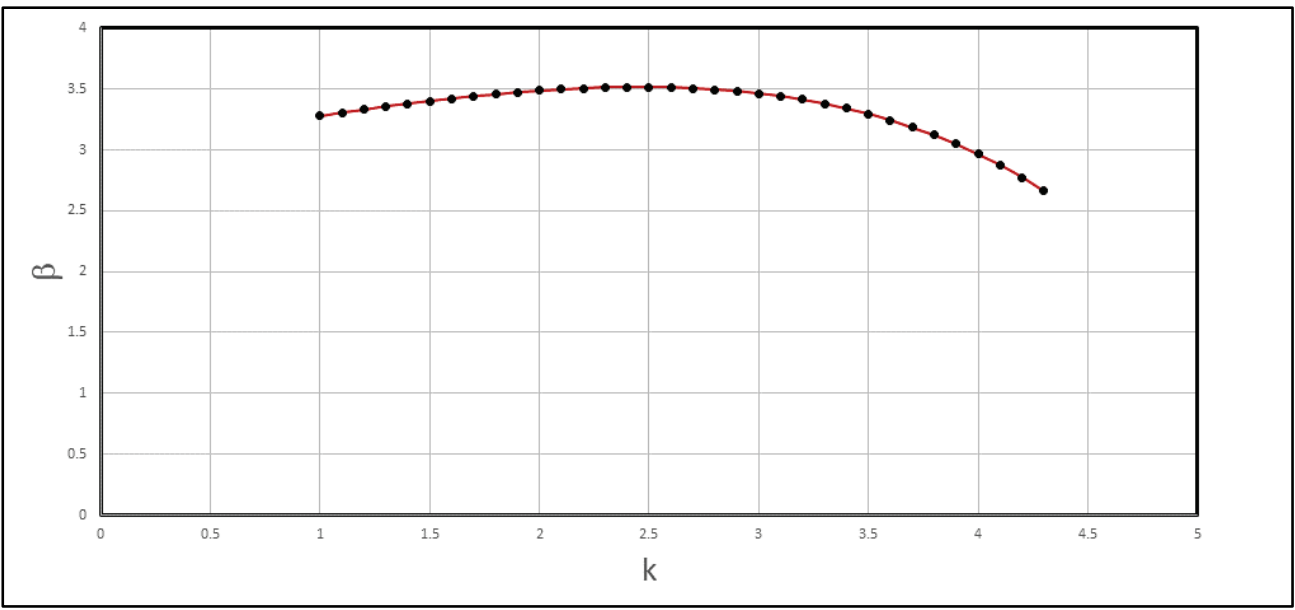


Table C-6. Reliability Index $\beta$ as a function of the constant $k$ for the problem of a reinforced concrete beam with its capacity defined by a nominal resisting moment of $337.68 \mathrm{kip}$-ft with a mean resisting moment of 378.31 kip-ft.

\begin{tabular}{|c|c|}
\hline $\mathrm{K}$ & $\boldsymbol{\beta}$ \\
\hline 1.8 & 3.458 \\
\hline 2 & 3.487 \\
\hline 2.1 & 3.498 \\
\hline
\end{tabular}

Recall that the Nowak (1999) numerical procedure discussed earlier in this section generated a maximum $\beta$ for $R-L$ of 3.52 with a $k$ value of 2.46 . Therefore, for an assigned $k$ value of 2 , a $\beta$ value of 3.487 is close to this maximum $\beta$ value from the numerical procedure.

\section{C.6 A Gaussian function superposition approach of numerically fitting normalized normal distribution functions to a non-normal pdf and computing a value for Reliability Index $\beta$}

In his 2015 Doctor of Philosophy dissertation, Seyed Hooman Ghasemi (Ghesemi 2015) proposed using a series of Gaussian normalized Normal distribution functions to approximate a non-normal PDF safety margin $(R$ $L$ ) distribution. Dr. Ghasemi's dissertation committee was chaired by Professor Andrzej Nowak. Professor Nowak has been a leader in LRFD research with application in bridge design. By their approach, a series of $n$ overlapping scaled Gaussian functions have scaled PDFs that can be summed to approximate the shape of the non-normal PDF safety margin and have a cumulative area of the approximated curve that approaches 1.0 for $\mathrm{CDF}$ integration. The reliability index value can be determined from the series solution from the reliability indices for each of the individual Gaussian functions. Because a normal distribution function is being used for each term of the Gaussian series, a reliability index value may also be conveniently computed for each term. Dr. Ghasemi provided the relationship between the reliability index values computed for each of the $n$ terms and the resulting single $\beta$ value representative of the summed distribution for all $n$ terms. Since the resulting PDF for the summed $n$ Gaussian PDF distributions fits the original non-normal PDF, their resulting $\beta$ value is representative of the non-normal distribution and may be used in reliability and any subsequent assessments of load and resistance (i.e., partial safety) factors, $\gamma$ and $\phi$, respectively. 
The series superposition of generalized Gaussian function used for each term in the series of $n$ terms is given as

$$
f\left(x_{i}\right)=\sum_{i=1}^{n} a_{i} * \frac{1}{\sqrt{2 * \pi * \sigma^{2}}} * e^{\frac{(x-\mu)^{2}}{2 * \sigma^{2}}}
$$

The Reliability Index is defined based on the summation of the reliability indices for each of the normal distributions numerically fitted to the safety margin $(R-L)$ data during this numerical superposition fitting process. Values for the amplitude scale constants $a_{i}$, mean values $\mu_{i}$, standard deviations $\sigma i$, and reliability index values $\beta$ i for all $n$ series terms are adjusted during the best-fit numerical process using a Python numerical fit optimization library function minimize (scipy.optimize.minimize). This library function chooses between several optimization numerical methods to approach the solution.

$$
\beta=\sum_{i}^{n}\left(a_{i} * \beta_{i}\right)
$$

This equation simplifies to

$$
\beta=\sum_{i}^{n}\left(a_{i} * \frac{\mu_{i}}{\sigma_{i}}\right)
$$

Note that for each Gaussian term added, a set of three constants are to be defined by the numerical fitting procedure of the safety margin $(R-L)$ data during the course of numerical analysis. Python language-based software was written and a program was developed to facilitate the numerical bestfit computational process of defining and constructing the individual Gaussian terms and the series superposition PDF to the non-normal PDF of safety margin $(R-L)$ data points.

Recall that in the reinforced concrete beam example problem of Section C.2, the flexural resisting moment was defined as a lognormal distribution with a nominal moment of $311.11 \mathrm{kip}-\mathrm{ft}$, bias factor of 1.12 and a $C O V_{R}$ of o.14. The corresponding mean value for resisting moment equals 348.44 kip-ft by Equation C.19. The nominal value of the dead load moment is 200 kip-ft. The load distribution is normal with a dead load bias factor of 1.05 and $C O V_{D L}$ of 0.1. The corresponding mean value for dead load moment equals 210 kip-ft by Equation C.14. The nominal values for the resisting moments are calculated from samples in the real-world space, but to create samples in the real-world space, a normal distribution in logarithmic space 
is used and for sampling and the values are converted to real space. The standard deviation and mean of the normal distribution in logarithmic space are calculated as

$$
\begin{gathered}
\sigma_{\ln R}=\sqrt{\ln \left(1+\frac{\sigma_{R}^{2}}{\mu_{R}^{2}}\right)} \\
\mu_{\ln R}=\ln \left(\mu_{R}\right)-0.5 \sigma_{\ln R}^{2}
\end{gathered}
$$

The safety margin $(R-L)$ PDF data was determined by simulation, gathering 10 million samples from the resistance and load distributions. The samples were subtracted to generate the $(R-L)$ samples. The results from this process is shown in Figure C-3, where the yellow curve is the safety margin and values below 0.0 for the safety margin curve are unsatisfactory. The PUP value is therefore the integrated probability of the yellow curve for values below o.o. Using the parameters in the previous paragraph, Figure $\mathrm{C}-4$ shows the simulated safety margin $(R-L)$ distribution.

Figure C-3. Computed safety margin PDF, PDF for a normal load PDF defined with a mean of 210 kip-ft, and a PDF for a lognormal resistance PDF defined by a nominal resisting moment of 311.11 kip-ft with a mean resisting moment of 348.44 kip-ft.

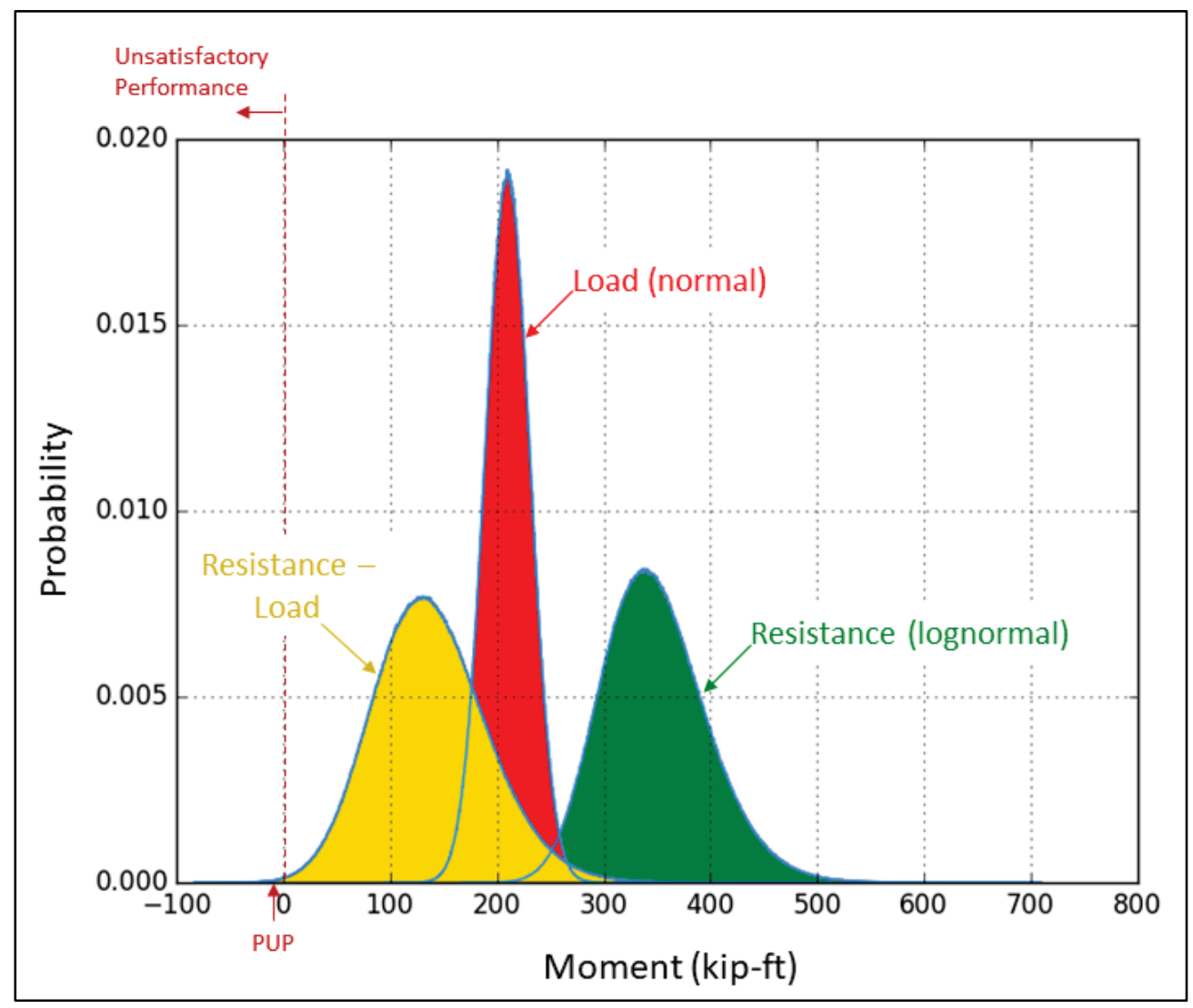


Through trial and error, it was found that a series of seven Gaussian function terms results in a good fit for the distribution curve of the safety margin $(R-L)$ PDF data. When the number of functional terms was less than seven, the changes in curvature for the safety margin PDF were not followed as well as the seven term Gaussian fit. With more functional terms than seven, the variation in curvature became too large resulting in a multimodal curve rather than the single mode simulation curve. This result is virtually guaranteed because each functional term curve adds three more variables to the fitness function (the function variables of $a_{i}, \mu_{i}$, and $\sigma_{i}$ times the number of functional terms). Initial values for the functional term variables were chosen with small standard deviations, $a_{i}$ 's that summed to 1.0, and separation in the means to encourage movement.

The individual sets of $a_{i}, \mu_{i}, \sigma_{i}$ and $\beta_{i}$ values for each of the seven Gaussian terms that were superimposed, resulted in the best fit of the nonlinear $(R$ $L)$ PDF are summarized in Table C-7. The sampled distribution is shown in Figure $\mathrm{C}-4$, and the individual terms forming the summed curve fit distribution are shown in Figure C-5.

Table C-7. Computed values of series constant coefficients, means, standard deviations, and reliability indices for each of the seven Gaussian functions for the problem of a reinforced concrete beam with its capacity defined by a nominal resisting moment of 311.11 kip-ft with a mean resisting moment of $348.44 \mathrm{kip}-\mathrm{ft}$.

\begin{tabular}{|l|l|l|l|l|}
\hline $\begin{array}{l}\text { Series } \\
\text { Term \# }\end{array}$ & $\boldsymbol{A}_{\boldsymbol{l}}$ & $\boldsymbol{\mu}_{\boldsymbol{l}}$ & $\boldsymbol{\sigma}_{\boldsymbol{l}}$ & $\boldsymbol{\beta}_{\boldsymbol{l}}$ \\
\hline $\mathbf{1}$ & 0.000902 & 131.166763 & 13.459697 & 9.745150 \\
\hline $\mathbf{2}$ & 0.302227 & 152.789236 & 39.598064 & 3.858503 \\
\hline $\mathbf{3}$ & 0.178650 & 108.349004 & 41.063404 & 2.638578 \\
\hline $\mathbf{4}$ & -0.000376 & 164.436394 & 11.450387 & 14.360772 \\
\hline $\mathbf{5}$ & -0.001067 & 92.533972 & 16.565957 & 5.585791 \\
\hline $\mathbf{6}$ & 0.278710 & 171.082874 & 57.469848 & 2.976915 \\
\hline $\mathbf{7}$ & 0.241486 & 104.461490 & 37.327819 & 2.798489 \\
\hline
\end{tabular}


Figure C-4. Resulting simulation PDF for the $R-L$ distribution given a lognormal resistance distribution (nominal resisting moment of $311.11 \mathrm{kip}-\mathrm{ft}$ and $C O V_{R}=0.14$ ) and a normal load distribution (nominal load moment of 200.00 kip-ft and COVDL $=0.1$ ).

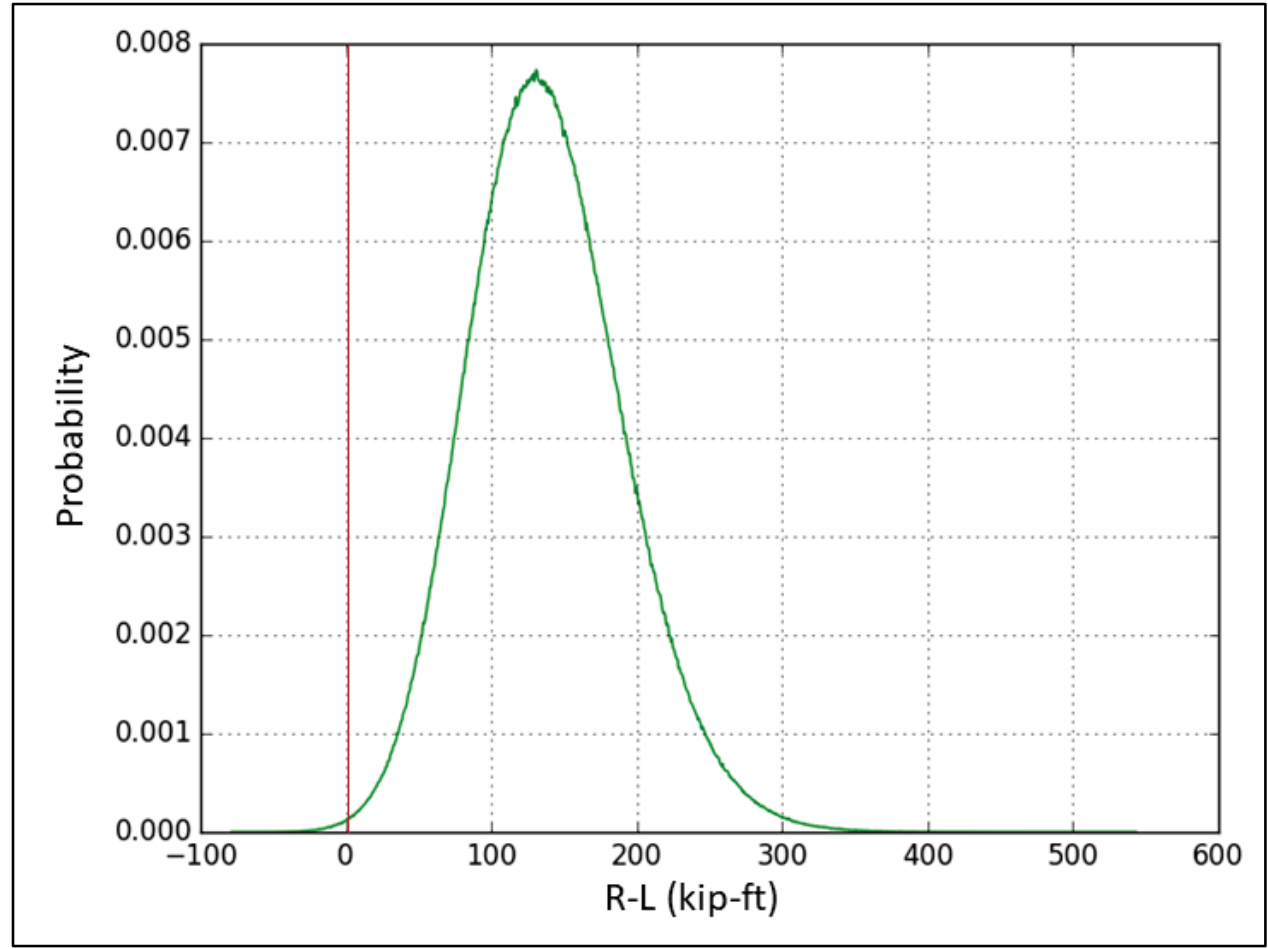

Figure C-5. Resulting simulation PDF for the $R-L$ distribution (given nominal resisting moment of 311.11 kip-ft) with curve fit by summing seven fractional normal curves.

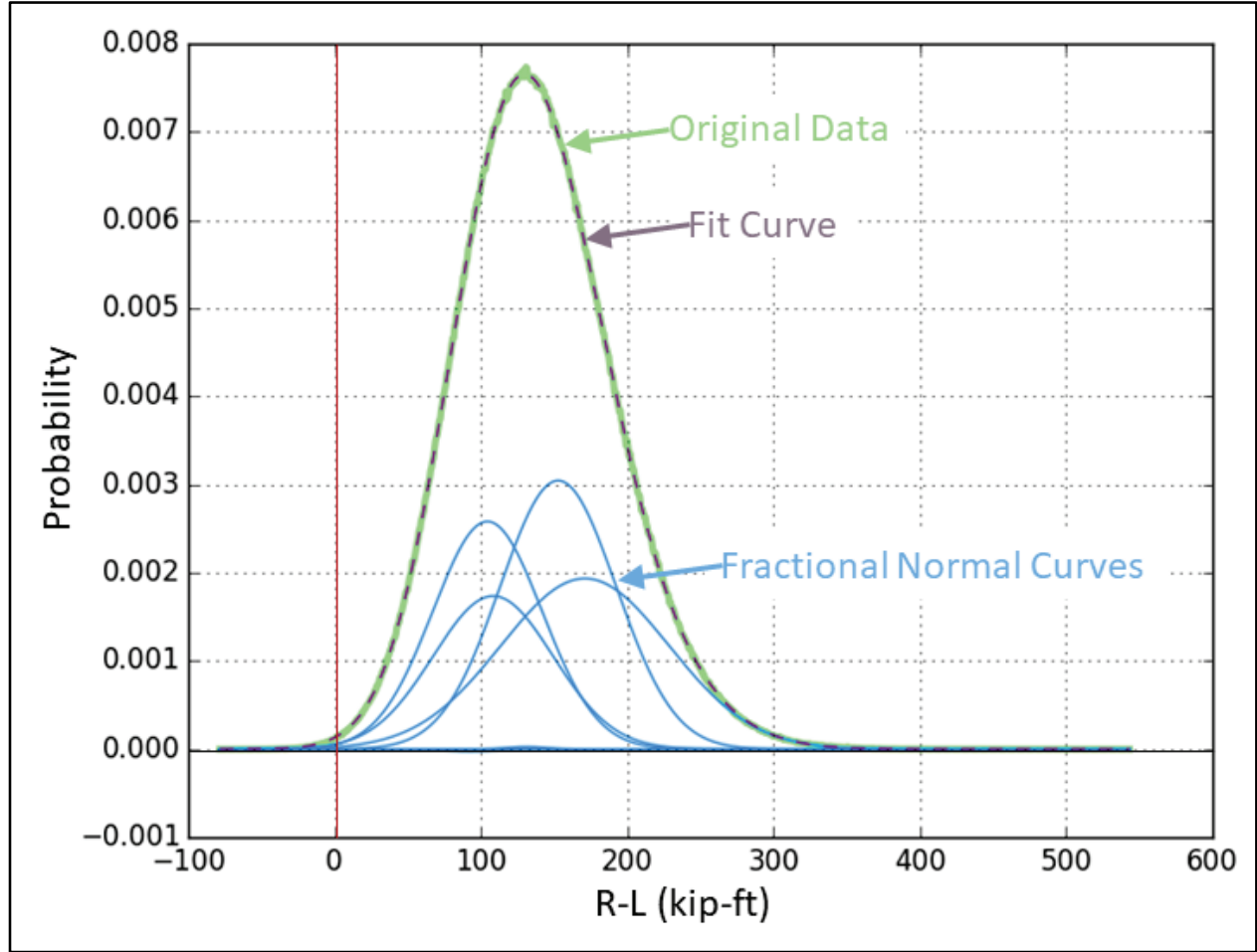


To correctly model the probability curve, it is imperative that the sum of the fractional normal areas should equal to 1.0 because this matches the total cumulative probability. The area of each fractional normal curve is $a_{i}$ because the scale factor is applied to a unit area standard normal curve. Summing the $a_{i}$ column of Table C-7 returns a value of 1.000532 , which is very close to the probability area.

From equation C.56 and Table C-7, one can calculate a value for the PUP $\beta$ for the fit curve. This combined PUP $\beta$ value is 3.140449. The residual error of this curve fit was 0.o, indicating an excellent fit over the entire domain. More importantly, the coefficient of determination $\left(R^{2}\right)$ give a fitness value of $95.5 \%$ for the PUP region.

For the second example, recall that for the reinforced concrete beam example problem of Section C.3, the flexural resisting moment was defined as a lognormal distribution with a nominal moment of $337.68 \mathrm{kip}-\mathrm{ft}$, bias factor of 1.12, and a $C O V_{R}$ of 0.14. The corresponding mean value for resisting moment equals $378.31 \mathrm{kip}-\mathrm{ft}$ by Equation C.19. The nominal value of the dead load moment is 200 kip-ft. The load distribution is normal with a dead load bias factor of 1.05 and $C O V_{D L}$ of 0.1. The corresponding mean value for dead load moment equals 210 kip-ft by Equation C.14. Using the parameters in this paragraph, Figure C-6 shows the simulated safety margin $(R-L)$ distribution.

A series of seven Gaussian function terms were used, resulting in a good fit of the distribution curve of the safety margin $(R-L)$ PDF data. The individual sets of $a_{i}, \mu_{i}, \sigma_{I}$, and $\beta_{i}$ values for each of the seven Gaussian terms that were superimposed resulted in the best fit of the nonlinear $(R-L)$ PDF are summarized in Table C-8. The sampled distribution is shown in Figure $\mathrm{C}-6$, and the individual terms forming the summed curve fit distribution are shown in Figure C-7. 
Table C-8. Computed values of series constant coefficients, means, standard deviations and reliability indices for each of the seven Gaussian functions for the problem of a reinforced concrete beam with its capacity defined by a nominal resisting moment of $337.68 \mathrm{kip}-\mathrm{ft}$ with a mean resisting moment of $378.31 \mathrm{kip}-\mathrm{ft}$.

\begin{tabular}{|l|l|l|l|l|}
\hline $\begin{array}{l}\text { Series } \\
\text { Term \# }\end{array}$ & $\boldsymbol{A}_{\boldsymbol{l}}$ & $\boldsymbol{\mu}_{\boldsymbol{l}}$ & $\boldsymbol{\sigma}_{\boldsymbol{l}}$ & $\boldsymbol{\beta}_{\boldsymbol{l}}$ \\
\hline $\mathbf{1}$ & 0.126179 & 161.415548 & 15.599337 & 10.347590 \\
\hline $\mathbf{2}$ & -0.018637 & 175.203552 & 16.873393 & 10.383421 \\
\hline $\mathbf{3}$ & -0.045216 & 147.748934 & 16.670027 & 8.863149 \\
\hline $\mathbf{4}$ & 0.083196 & 188.816040 & 17.711134 & 10.660867 \\
\hline $\mathbf{5}$ & 0.120355 & 134.193684 & 17.058013 & 7.866900 \\
\hline $\mathbf{6}$ & 0.403193 & 208.227268 & 42.576387 & 4.890675 \\
\hline $\mathbf{7}$ & 0.323917 & 115.431851 & 39.080976 & 2.953658 \\
\hline
\end{tabular}

Figure C-6. Resulting simulation PDF for the $R-L$ distribution given a lognormal resistance distribution (nominal resisting moment of 337.68 kip-ft and $C O V_{R}=0.14$ ) and a normal load distribution (nominal resisting moment of $200.00 \mathrm{kip}-\mathrm{ft}$ and $C O V_{D L}=0.1$ ).

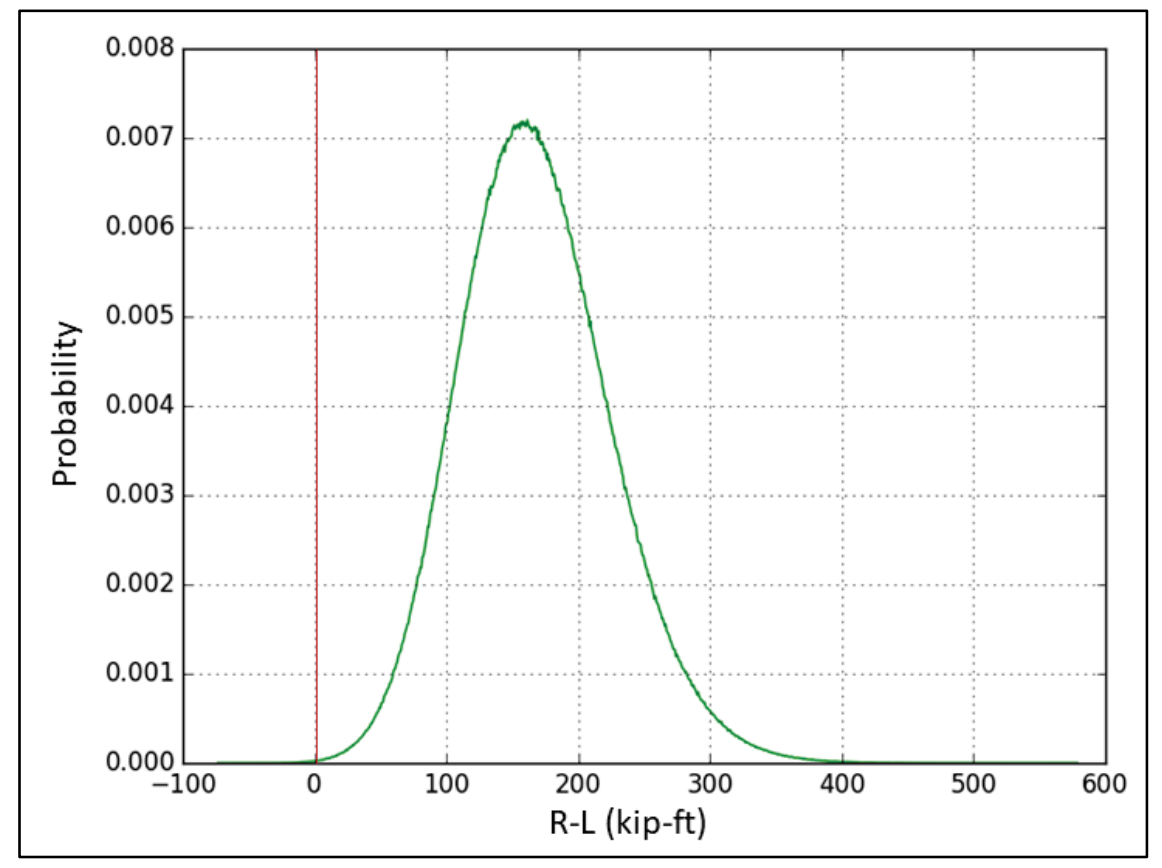


Figure C-7. Resulting simulation PDF for the $R-L$ distribution (given nominal resisting moment of $337.68 \mathrm{kip}-\mathrm{ft}$ ) with curve fit by summing seven fractional normal curves.

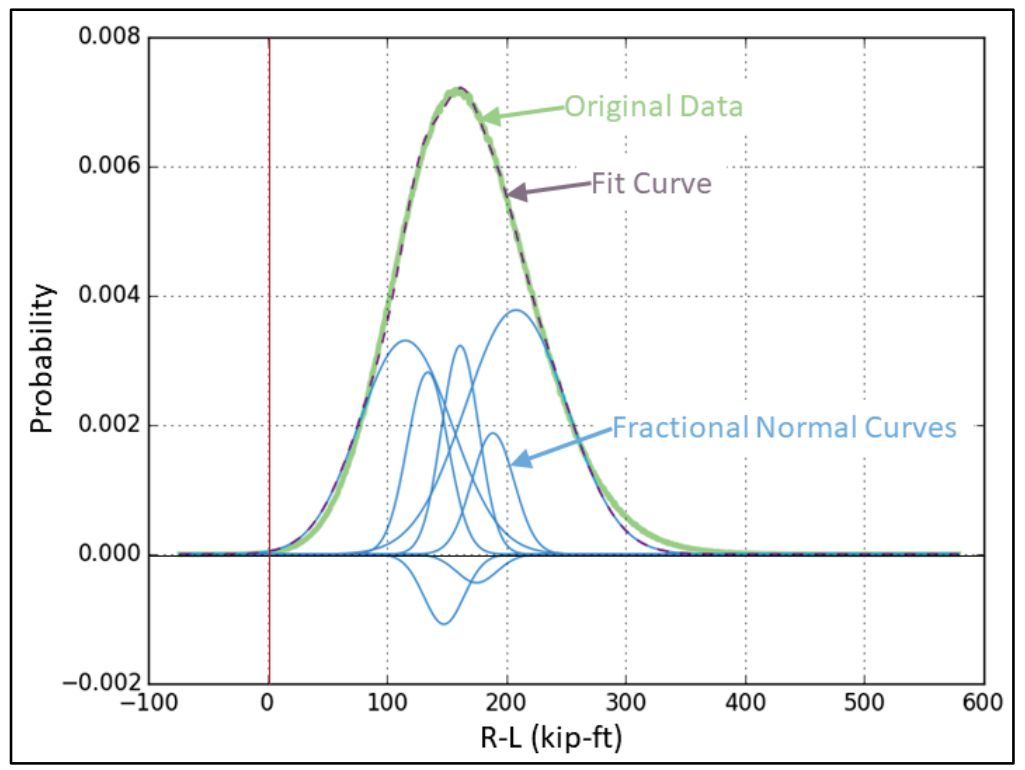

To correctly model the probability curve, it is imperative that the sum of the fractional normal areas should equal to 1.0 because this matches the total cumulative probability. The area of each fractional normal curve is $a_{i}$ because the scale factor is applied to a unit area standard normal curve. Summing the $a_{i}$ column of Table C-8 returns a value of 0.992978 , which is very close to the probability area.

From Equation C.56 and Table C-8, one can calculate a value for the PUP $\beta$ for the fit curve. This combined PUP $\beta$ value is 5.473765. The residual error for this fit curve was very low with a value of 0.000007 , although there seems to be issues at the peak and on the right side of the data, which could have an effect on the $\beta$ calculation. Note that the error occurs mostly in the tails of the distributions, as those areas affect the PUP calculation. An attempt was made to calculate $R^{2}$ for the PUP region, but the data in the tails of the PUP region had extremely low values of magnitude. The low magnitude of the values in this region is explained by $\beta=5.473765$.

\section{C.7 Summary and conclusions}

Four numerical procedures were investigated, and a numerical relationship of the load and resistance factors was identified in this appendix. These numerical procedures were derived to handle the specific example problem with a log-normal distribution for the resistance term and a normal distribution for load. Section C.2 investigated a procedure that, when 
presented with load and resistance PDF curves, would generate load and resistance factors (without bias) that scale the PDF curves so that the combined PUP result would have a CDF value equivalent to a normal distribution CDF at $\beta_{\text {target. }}$ Section C.3 was a quick numerical exploration of the fact that keeping the ratio between the load and resistance factors the same will result in the same resulting value for $\beta$ because the ratio of scaled resistance to scaled load determines the PUP result. Sections C.4 through C. 6 explore ways of calculating $\beta$ given different input values that describe the load and resistance PDF curves.

Two example problems were included based upon a trial design of a singly reinforced beam with an unfactored dead load bending moment, $M_{D L}$, set equal to $200 \mathrm{kip}-\mathrm{ft}$ for a beam with a width of $12 \mathrm{in}$. and a depth $(d)$ to center of reinforcement of 28 in. This example was taken from a 2016 USACE LRFD study (unpublished). This appendix outlines the complete numerical procedure to follow to obtain appropriate values for load and resistance factors for a prescribed $\beta_{\text {target }}$ value as well as a supplemental procedure to compute a load factor consistent with the ACI prescribed resistance factor $\phi$ value maintaining factor relationships computed for a prescribed $\beta_{\text {target }}$ value, so long as the mean load value remain unaltered. Table C-9 shows the difference of effect between the ACI/ASCE load and resistance factors and the factors computed by using the Section C.2 procedure with $\beta$ target $=3$.o. The third row shows the effect of using the Section C.3 ratio with the input ACI resistance factor (0.9) to change the load factor. The final row shows the results of the Section C.5 (Equations C.51 and C.52) factor calculations.

For the following tables, $\lambda_{L}$ is the load bias factor, $N_{L}$ is the nominal load, $\mu_{L}$ is the mean load, $C O V_{L}$ is the coefficient of variation for load, $\gamma_{L}$ is the load factor, $\phi_{R}$ is the resistance factor, $\lambda_{R}$ is the resistance bias factor, $N_{R}$ is the nominal resistance, $\mu_{R}$ is the mean resistance, and $C O V_{R}$ is the coefficient of variation for resistance.

Table C-9. Load and resistance factors and the effect on resistance.

\begin{tabular}{|l|l|l|l|l|l|l|l|l|l|l|l|}
\hline & $\lambda_{L}$ & $N_{L}$ & $\mu_{L}$ & $\operatorname{COV}_{L}$ & $\gamma_{L}$ & $\phi_{R}$ & $\gamma_{L} / \phi_{R}$ & $\lambda_{R}$ & $N_{R}$ & $\mu_{R}$ & $\operatorname{COV}_{R}$ \\
\hline $\mathrm{ACl}$ & 1.05 & 200.0 & 210.0 & 0.1 & 1.4 & 0.9 & 1.556 & 1.12 & 311.11 & 348.44 & 0.14 \\
\hline C.2 & 1.05 & 200.0 & 210.0 & 0.1 & 1.21595 & 0.720173 & 1.688 & 1.12 & 337.68 & 378.21 & 0.14 \\
\hline C.3 & 1.05 & 200.0 & 210.0 & 0.1 & 1.519586 & 0.9 & 1.688 & 1.12 & 337.68 & 378.21 & 0.14 \\
\hline C.5 & 1.05 & 200.0 & 210.0 & 0.1 & 1.26 & 0.806 & 1.563 & 1.12 & 312.66 & 350.17 & 0.14 \\
\hline
\end{tabular}


The consistency between computed $\gamma$ and $\phi$ values in Example C. 1 for the specified $\beta$ target value of 3.0 was verified by the second numerical procedure in Example C.2. This Example C.1 calculation of $\phi$ and $\gamma$ resulted in different values than the values reported from the 2016 USACE LRFD study (unpublished). In Example C.2, the calculated values were shown to be more accurate than the values from the 2016 USACE LRFD study (unpublished).

These examples were carried forward into the Section C.4, Section C.5, and Section C.6 methods of $\beta$ computation. The Section C.6 method was explicitly supported by simulation runs with 10,000,000 samples for both the load and resistance from the defined curves.

Table $\mathrm{C}-10$ shows the results of using the various computed load and resistance factors to calculate $\beta$ with the numerical procedure of Section C.4. This method seems to verify the results of the Section C.2 and C.3 computations, where $\beta$ target was set to 3.0.

Table C-10. Applying Section C.4 calculation of $\beta$ using the various load and resistance factors.

\begin{tabular}{|l|l|l|l|l|l|l|}
\hline Method & $N_{L}$ & $\gamma_{L}$ & $\phi_{R}$ & $\gamma_{L} / \phi_{R}$ & $N_{R}$ & Resultant $\beta$ \\
\hline ACI & 200.0 & 1.4 & 0.9 & 1.556 & 311.11 & 2.614 \\
\hline C.2 & 200.0 & 1.21595 & 0.720173 & 1.688 & 337.68 & 2.981 \\
\hline C.3 & 200.0 & 1.519586 & 0.9 & 1.688 & 337.68 & 2.981 \\
\hline C.5 & 200.0 & 1.26 & 0.806 & 1.563 & 312.66 & 2.636 \\
\hline
\end{tabular}

Table $\mathrm{C}-11$ shows the results of using the various computed nominal resistances computed from load and resistance factors to calculate $\beta$ with the numerical procedure of Section C.5. This method contradicts the C.4 method results, with the load and resistance factors computed from Section C.2 and C.3 procedures, which are coincident, giving a much more conservative value of $\sim 3.5$ for $\beta$. The load and resistance values for the factors given by ACI/ASCE and the Section C.5 equations is closer to $\beta$ target $=3.0$. The computed value for $k$ was derived from the last iteration design point $R^{*}$ using equation C.43. 
Table C-11. Calculating the maximum $\beta$ using the Section C.5 procedure given nominal resistances based on computed load and resistance factors.

\begin{tabular}{|l|l|l|l|l|l|l|l|}
\hline Method & $N_{R}$ & $\lambda_{R}$ & COV $_{R}$ & $\mu_{L}$ & $\sigma_{L}$ & $\beta$ & $k$ \\
\hline ACI & 311.11 & 1.12 & 0.14 & 210.0 & 21.0 & 3.016 & 2.157 \\
\hline C.2 and C.3 & 337.68 & 1.12 & 0.14 & 210.0 & 21.0 & 3.516 & 2.455 \\
\hline C.5 & 312.66 & 1.12 & 0.14 & 210.0 & 21.0 & 3.046283 & 2.175218 \\
\hline
\end{tabular}

Section C.6 involves fitting a set of fractional Gaussian distributions to the resulting resistance minus load distribution $(R-L)$ safety margin curve. In order to determine this curve, a set of Monte Carlo samples using the distributions of resistance and load are drawn and subtracted from each other. The resulting points for each distribution are then binned into a histogram to determine probabilities, resulting in curves similar to Figure $\mathrm{C}-3$. The $P U P$ value can be determined from the $R-L$ curve by integrating the portion of the curve with a moment below zero. A resultant $\beta$ value is computed from the PUP value by applying the inverse CDF function for a Gaussian Normal distribution to the PUP. Table $\mathrm{C}-12$ shows the results using the different load and resistance factors.

Table C-12. Results of $10 \mathrm{M}$ simulations being performed using procedural load and resistance factors.

\begin{tabular}{|l|l|l|l|l|}
\hline Method & $\gamma_{L}$ & $\phi_{R}$ & PUP & $\begin{array}{l}\text { Resultant } \\
\beta\end{array}$ \\
\hline ACI & 1.4 & 0.9 & 0.001441 & 2.98 \\
\hline C.2 & 1.21595 & 0.720173 & 0.000239 & 3.493 \\
\hline C.3 & 1.519586 & 0.9 & 0.000239 & 3.493 \\
\hline C.5 & 1.26 & 0.806 & 0.001286 & 3.015 \\
\hline
\end{tabular}

When the fractional Gaussian distributions have been determined that sum to a proper curve with low error that approximates the $R-L$ curve, Equation C.56 can be used to determine the $\beta$ value for a normal distribution that has the same CDF at the response surface where the safety margin $R-L=0$. Table $\mathrm{C}-13$ shows the results of these $\beta$ calculations for a low-error curve fit with seven distributions. Note that the initial seeded values for the fractional Gaussian distributions can play a large part in the amount of error in the fit and in the final $\beta$ calculation. This may be better controlled if the values for the fractional Gaussian distributions were bounded. The initial values for the fractional curves were adjusted to give a coefficient of determination, $R^{2}$, value for each summed curve of at least $95 \%$ of the original values. The resultant $\beta$ s reveal a weakness of this procedure when 
the fractional curve values were not bounded. Although the fitness curve has an area of one, which matches the simulation distribution, portions of the curve, especially in the tails, do not have good properties because individual fractional distribution curves can have negative area. This affects the CDF calculation, especially if the summed curve drops below zero in the integrated region.

Table C-13. Results of $10 \mathrm{M}$ simulations being performed using procedural load and resistance factors.

\begin{tabular}{|l|l|l|l|}
\hline Method & $\gamma L / \phi_{R}$ & $\mathrm{R}^{2}$ & Resultant $\boldsymbol{\beta}$ \\
\hline $\mathrm{ACl}$ & 1.556 & 0.955 & 3.14 \\
\hline C.2 and C.3 & 1.688 & 0.993 & 5.47 \\
\hline C.5 & 1.563 & 0.977 & 2.46 \\
\hline
\end{tabular}

This appendix introduced three methods for computing load and resistance factors. The first method is using engineering standards. The second method is using the Section C.2 numerical method, which establishes a ratio for the Section C.3 ratio method. Finally, the third method is using the Section C.5 numerical procedure, which calculates a $k$ value (for the max $\beta$ ) that can be applied in Equations C.51 and C.52.

This appendix also introduced techniques for computing $\beta$ given load and resistance factors either directly or indirectly. The Section C.4 numerical procedure attempts to iterate to find a maximum $\beta$ given resistance and load distribution descriptions using Rackwitz and Fiessler $(1976,1978)$ normal curve approximates. The Section C.5 numerical procedure computes the maximum $\beta$ and its associated $k$ value. Substituting this $k$ value into Equation C.53 will return this maximum $\beta$ value, also. Section C.6 attempts to indirectly calculate the $\beta$ value for a non-normal distribution by summing fractional Gaussian curves to fit the non-normal distribution with low error. The fractional curve properties are used in Equation C.56 to determine the $\beta$ value for a non-normal distribution. The authors also did a Monte-Carlo simulation with a large number of samples on the resistance and load curves and calculated the $R-L$ curve with binned histograms. The PUP value was then calculated by integrating probabilities for the resulting distribution up to the safety margin $R-L=0$ response surface and an inverse normal distribution $\mathrm{CDF}$ calculation was used to get a transformed Gaussian Normal space $\beta$ value. 
In conclusion, given two full distribution descriptions, the simulation method (with enough samples) and the Section C.5 procedural method can determine an accurate PUP value either directly or through a normal distribution $\mathrm{CDF}$ calculation from a calculated $\beta$. Based on extensive series of trial and error computations, the Section C. 6 method was found to be an interesting mathematical exercise but has too many variables and needs restraint. The Section C. $2 \beta$ calculation method for non-normal distributions requires small steps using normal distributions mapped to the non-normal distribution at a given design point (Rackwitz and Fiessler 1976,1978 ), which can have problems with accuracy in the tails region, as shown in the results tables in this section of the appendix.

The Section C. 2 procedural method is the only method that attempts to numerically establish load and resistance factors that will scale the positions of existing distributions so that the safety margin $(R-L)$ response surface will have a PUP value that corresponds to a normal distribution $\beta_{\text {target }} \mathrm{CDF}$ value. Unfortunately, this procedure is attempting to find a value in the tail of the distribution and therefore has accuracy problems that are revealed in the results tables in this section of the appendix. 


\section{Appendix D: Calculations of Soil Bearing Capacity for Limit State Definition}

\section{D.1 Introduction: bearing defined}

The form of the generalized bearing capacity equation used for the example problem is given as

$$
q=\zeta_{c} \zeta_{c d} \zeta_{c i} \zeta_{c t} \zeta_{c g} c N_{c}+\zeta_{q} \zeta_{q d} \zeta_{q i} \zeta_{q t} \zeta_{q g} q_{0} N_{q}+\frac{\zeta_{\gamma} \zeta_{\gamma d} \zeta_{\gamma i} \zeta_{\gamma t} \zeta_{\gamma g} B \gamma N_{\gamma}}{2}
$$

where

$Q=$ vertical component of the ultimate unit bearing capacity of the foundation (pressure)

$N_{c}, N_{q}, N_{\gamma}=$ base bearing capacity factors

$\zeta_{c}, \zeta_{q}, \zeta_{\gamma}=$ shape factors

$\zeta_{c d}, \zeta_{q d}, \zeta_{\gamma d}=$ embedment factors

$\zeta_{c i}, \zeta_{q i}, \zeta_{Y I}=$ inclination factors

$\zeta_{c t}, \zeta_{q t}, \zeta_{t t}=$ base tilt factors

$\zeta_{c g}, \zeta_{q g}, \zeta_{\gamma g}=$ ground slope factors

$C=$ cohesion

$\gamma=$ unit weight of the soil

$B_{e}=$ effective base width

$L=$ effective base length (for a 2-D section evaluation, a value of 1.0 is used)

$q o=$ effective overburden pressure on a plane passing through the base of the footing.

Because the computations used for Sliding and Base Area in Compression (Overturning) limit states already provide the effective vertical force exhibited by the structural wedge, which acts directly against the base, the bearing capacity is converted to an ultimate resisting force for the effective base width (for the 2-D slice), which is the width of the base in contact with the foundation soil. This width will be affected by uplift and overturning forces that cause a gap to form between the foundation soil and the foundation.

$$
R_{\text {Bearing }}=B_{e} q
$$


This is the ultimate bearing capacity force for the entire foundation, and if the vertical force acting on the structural wedge exceeds this value, the structural soil provides unsatisfactory performance. Figure D-1 shows a 2-D cross section of a complex T-Wall geometry with enough input information to determine the resistance to bearing failure.

Figure D-1. A geometry view of a complex T-Wall configuration with definition of the geometry necessary to compute bearing capacity.

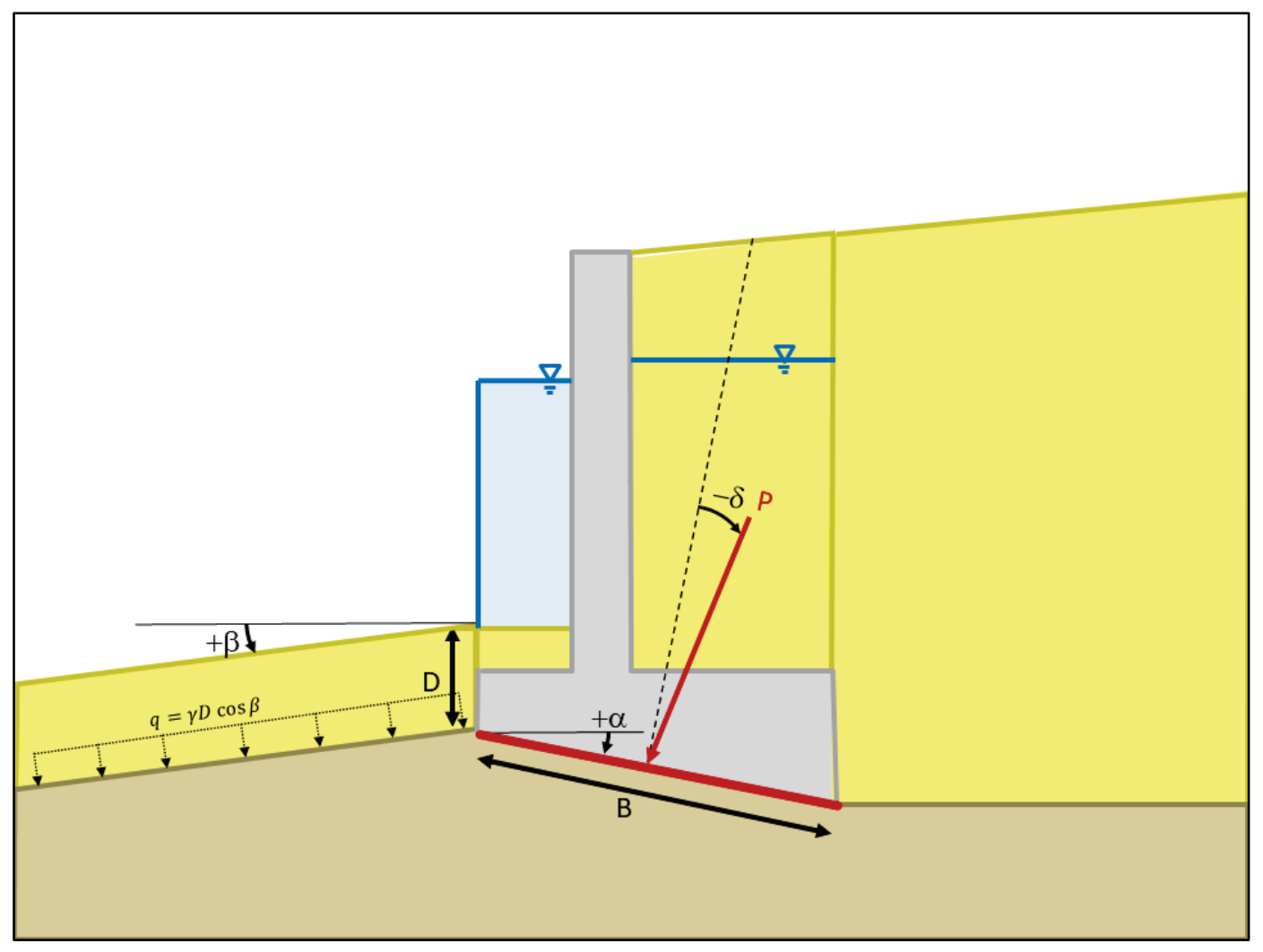

\section{D.2 Base bearing capacity factors $\left(\mathbf{N}_{c}, \mathbf{N}_{q}, \mathbf{N}_{\gamma}\right)$}

The base bearing capacity factors used in this program are the ones developed by Meyerhof (1963) for a shallow horizontal strip footing under vertical load. These values are conditionally based on the value of the foundation soil Interface Friction Angle $\phi$ :

$$
\begin{gathered}
N_{c}=\left(N_{q}-1\right) \cot \phi \\
N_{q}=e^{\pi \tan \phi} N_{\phi} \\
N_{\gamma}=\left(N_{q}-1\right) \tan 1.4 \phi
\end{gathered}
$$


where

$$
N_{\phi}=\tan ^{2}\left(\frac{\pi}{4}+\frac{\phi}{2}\right)
$$

However, for $\phi=0^{\circ}$, Equation D.3 is indefinite, so the value is given as

$$
N_{c}=5.14
$$

\section{D.3 Shape factors $\left(\zeta_{\alpha}, \zeta_{q}, \zeta_{\gamma}\right)$}

The shape factors used in this program also use the relationships developed by Meyerhof (1963). These values are conditionally based on the value of the foundation soil Interface Friction Angle $\phi$ :

$$
\zeta_{c}=1.0+0.2 N_{\phi} \frac{B}{L}
$$

For $\phi=0^{\circ}$,

$$
\zeta_{q}=\zeta_{\gamma}=1.0
$$

For $\phi>10^{\circ}$,

$$
\zeta_{q}=\zeta_{\gamma}=1.0+0.1 N_{\phi} \frac{B}{L}
$$

For $0^{\circ}<\phi \leq 10^{\circ}$, a linear interpolation between Equations D.9 and D.10 (with $\phi=10.0$ ) is used.

\section{D.3 Embedment factors $\left(\zeta_{c d}, \zeta_{q d}, \zeta_{\gamma d}\right)$}

The embedment factors also use the relationships developed by Meyerhof (1963). These values are conditionally based on the value of the foundation soil Interface Friction Angle $\phi$ :

$$
\zeta_{c d}=1.0+0.2 \frac{D}{B} \tan \left(\frac{\pi}{4}+\frac{\phi}{2}\right)
$$

where $\mathrm{D}$ is the depth of embedment at the base of the footing.

For $\phi=0^{\circ}$,

$$
\zeta_{q d}=\zeta_{\gamma d}=1.0
$$


For $\phi>10^{\circ}$,

$$
\zeta_{q d}=\zeta_{\gamma d}=1.0+0.1 \frac{D}{B} \tan \left(\frac{\pi}{4}+\frac{\phi}{2}\right)
$$

For $0^{\circ}<\phi \leq 10^{\circ}$, a linear interpolation between Equations D.12 and D.13 (with $\phi=10.0$ ) is used.

\section{D.4 Inclination factors $\left(\zeta_{c l,} \zeta_{q l,} \zeta_{\gamma l}\right)$}

The inclination factors also use the relationships developed by Meyerhof (1963).

$$
\zeta_{c i}=\zeta_{q i}=\left(1-\frac{\delta}{90}\right)^{2}
$$

Where $\delta$ is the angle of inclination of the load from vertical as shown in Figure D-1.

For $\delta<=\phi$,

$$
\zeta_{\gamma i}=\left(1-\frac{\delta}{\phi}\right)^{2}
$$

For $\delta>\phi$,

$$
\zeta_{\gamma i}=0.0
$$

\section{D.5 Base tilt factors $\left(\zeta_{c t}, \zeta_{q t,} \zeta_{\gamma t}\right)$}

The base tilt factors use the relationships developed by Vesic (1975).

$$
\zeta_{q t}=\zeta_{\gamma t}=(1-\alpha \tan \phi)^{2}
$$

where $\alpha$ is the slope of the base of the footing as shown in Figure D-1.

For $\phi=0^{\circ}$,

$$
\zeta_{c t}=1-\left(2 \frac{\alpha}{\pi+2}\right)
$$

For $\phi>0^{\circ}$,

$$
\zeta_{c t}=\zeta_{q t}-\frac{1-\zeta_{q t}}{N_{c} \tan \phi}
$$




\section{D.6 Ground slope factors $\left(\zeta_{c g}, \zeta_{q g}, \zeta_{\gamma g}\right)$}

Ground slope factors are compute using the relationships developed by Vesic (1975).

$$
\zeta_{q g}=\zeta_{\gamma g}=(1-\tan \beta)^{2}
$$

where $\beta$ is the slope of the surface soil as shown in Figure D-1.

For $\phi=0^{\circ}$,

$$
\begin{gathered}
\zeta_{c g}=1-\left(2 \frac{\beta}{\pi+2}\right) \\
N_{\gamma}=-2 \sin \beta
\end{gathered}
$$

For $\phi>0^{\circ}$,

$$
\zeta_{c g}=\zeta_{q g}-\frac{1-\zeta_{q g}}{N_{c} \tan \phi}
$$

\section{D.7 Effective foundation dimensions}

An approximate method developed by Meyerhof (1963) is employed to adjust the foundation dimensions for eccentric loadings. For the strip footings employed in this report,

$$
B_{e}^{\prime}=B_{e}-2.0 e_{x}
$$




\section{Acronyms and Abbreviations}

1-D one-dimensional

2-D two-dimensional

5-D five-dimensional

ASD allowable stress design

AASHTO American Association of State Highway and Transportation Officials

ASD allowable stress design

ASM Advanced second moment

CASE Computer-Aided Structural Engineering

CDF cumulative distribution function

CHBDC Canadian Highway Bridge Design Code

COV coefficient of variation

FS factor(s) of safety

LRFD Load and Resistance Factor Design

LSF $\quad$ load scale factor

PDF probability density function

PUP Probability of Unsatisfactory Performance

$\mathrm{R} \& \mathrm{D} \quad$ research and development

$R_{S F} \quad$ resistance scale factor

SSI soil structure interaction

ULS Ultimate Limit States

USACE U.S. Army Corps of Engineers 
The public reporting burden for this collection of information is estimated to average 1 hour per response, including the time for reviewing instructions, searching existing data sources, gathering and maintaining the data needed, and completing and reviewing the collection of information. Send comments regarding this burden estimate or any other aspect of this collection of information, including suggestions for reducing the burden, to Department of Defense, Washington Headquarters Services, Directorate for Information Operations and Reports (0704-0188), 1215 Jefferson Davis Highway, Suite 1204, Arlington, VA 22202-4302. Respondents should be aware that notwithstanding any other provision of law, no person shall be subject to any penalty for failing to comply with a collection of information if it does not display a currently valid OMB control number. PLEASE DO NOT RETURN YOUR FORM TO THE ABOVE ADDRESS.

\begin{tabular}{l|l|l}
\hline $\begin{array}{l}\text { 1. REPORT DATE } \\
\text { March } 2021\end{array}$ & $\begin{array}{l}\text { 2. REPORT TYPE } \\
\text { Final Report }\end{array}$ & 3. DATES COVERED (From - To) \\
\hline
\end{tabular}

\section{TITLE AND SUBTITLE}

Load and Resistance Factors for Earth Retaining, Reinforced Concrete Hydraulic Structures Based on a Reliability Index $(\beta)$ Derived from the Probability of Unsatisfactory Performance (PUP): Phase 2 Study 5a. CONTRACT NUMBER

5b. GRANT NUMBER

5c. PROGRAM ELEMENT NUMBER

5d. PROJECT NUMBER

5e. TASK NUMBER

5f. WORK UNIT NUMBER

8. PERFORMING ORGANIZATION REPORT NUMBER

ERDC/ITL TR-21-1

3909 Halls Ferry Road

Vicksburg, MS 39180-6199

9. SPONSORING/MONITORING AGENCY NAME(S) AND ADDRESS(ES)

Navigation Systems (NavSys) Research Program

U.S. Army Engineer Research and Development Center

3909 Halls Ferry Road

Vicksburg, MS 39180-6199

12. DISTRIBUTION/AVAILABILITY STATEMENT

Approved for public release; distribution is unlimited.

\section{Funding Account Code U4375143; AMSCO Code 031391}

\section{ABSTRACT}

This technical report documents the second of a two-phase research and development (R\&D) study in support of the development of a combined Load and Resistance Factor Design (LRFD) methodology that accommodates geotechnical as well as structural design limit states for design of the U.S. Army Corps of Engineers (USACE) reinforced concrete, hydraulic navigation structures. To this end, this R\&D effort extends reliability procedures that have been developed for other non-USACE structural systems to encompass USACE hydraulic structures. Many of these reinforced concrete, hydraulic structures are founded on and/or retain earth or are buttressed by an earthen feature. Consequently, the design of many of these hydraulic structures involves significant soil structure interaction.

Development of the required reliability and corresponding LRFD procedures has been lagging in the geotechnical topic area as compared to those for structural limit state considerations and have therefore been the focus of this second-phase R\&D effort. Design of an example T-Wall hydraulic structure involves consideration of five geotechnical and structural limit states. New numerical procedures have been developed for precise multiple limit state reliability calculations and for complete LRFD analysis of this example T-Wall reinforced concrete, hydraulic structure.

\section{SUBJECT TERMS}

Concrete hydraulic structures, Geostatistics, Geotechnical engineering, Load and Resistance Factor Design (LRFD), Multiple limit states, Reinforced concrete hydraulic structures, Reliability analysis, Scale of fluctuation, Variance reduction factor, Soil-Structure Interaction (SSI), Structural engineering

\section{SECURITY CLASSIFICATION OF:}

\begin{tabular}{|l|c|l|}
\hline a. REPORT & b. ABSTRACT & c. THIS PAGE \\
Unclassified & Unclassified & Unclassified \\
& & \\
\hline
\end{tabular}

17. LIMITATION OF ABSTRACT

SAR
18. NUMBER OF PAGES

273 19a. NAME OF RESPONSIBLE PERSON Robert M. Ebeling

19b. TELEPHONE NUMBER (Include area code) 601-634-3458 